\title{
Systematic Investigation of the Scope of Transannular C-H Heteroarylation of Cyclic Secondary Amines for Synthetic Application in Medicinal Chemistry
}

\author{
Zhe Li ${ }^{\star *,+\neq}$, Michael Dechantsreiter ${ }^{\star, t}$, Sivaraman Dandapani ${ }^{\star, t}$ \\ ${ }^{\dagger}$ Department of Medicinal Chemistry, Biotherapeutic and Medicinal Sciences, Biogen, Cambridge, \\ MA, USA \\ ${ }^{\ddagger}$ Biogen Postdoctoral Scientist Program, Biogen, Cambridge, MA, USA
}

Corresponding Authors:

`Zhe Li (Z. L.) E-mail: zhe.li@biogen.com

*Michael Dechantsreiter (M. D.) E-mail: michael.dechantsreiter@biogen.com

*Sivaraman Dandapani (S. D.) E-mail: sivaraman@skyhawktx.com. During the preparation of this manuscript, S.D. moved to Skyhawk Therapeutics from Biogen Inc..

\section{SUPPORTING INORMATION}

\section{Table of Contents}

General Procedure for Initial Investigation of Transannular C-H Heteroarylation Reactions.......S1

General Procedure for Ligand Screening and Conditions Optimizations..........................S2

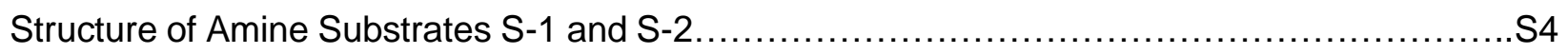

Unsuccessful Heteroaryl lodides in the Transannular C-H Heteroarylation Reactions.............S4

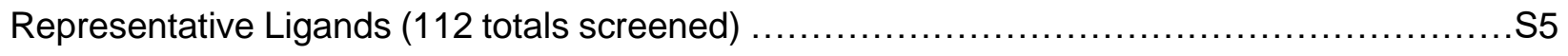

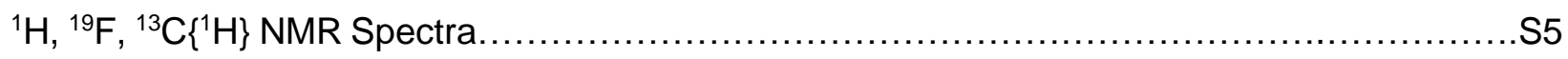


1. General Procedure for the Initial Investigation of Transannular C-H Heteroarylation Reactions.

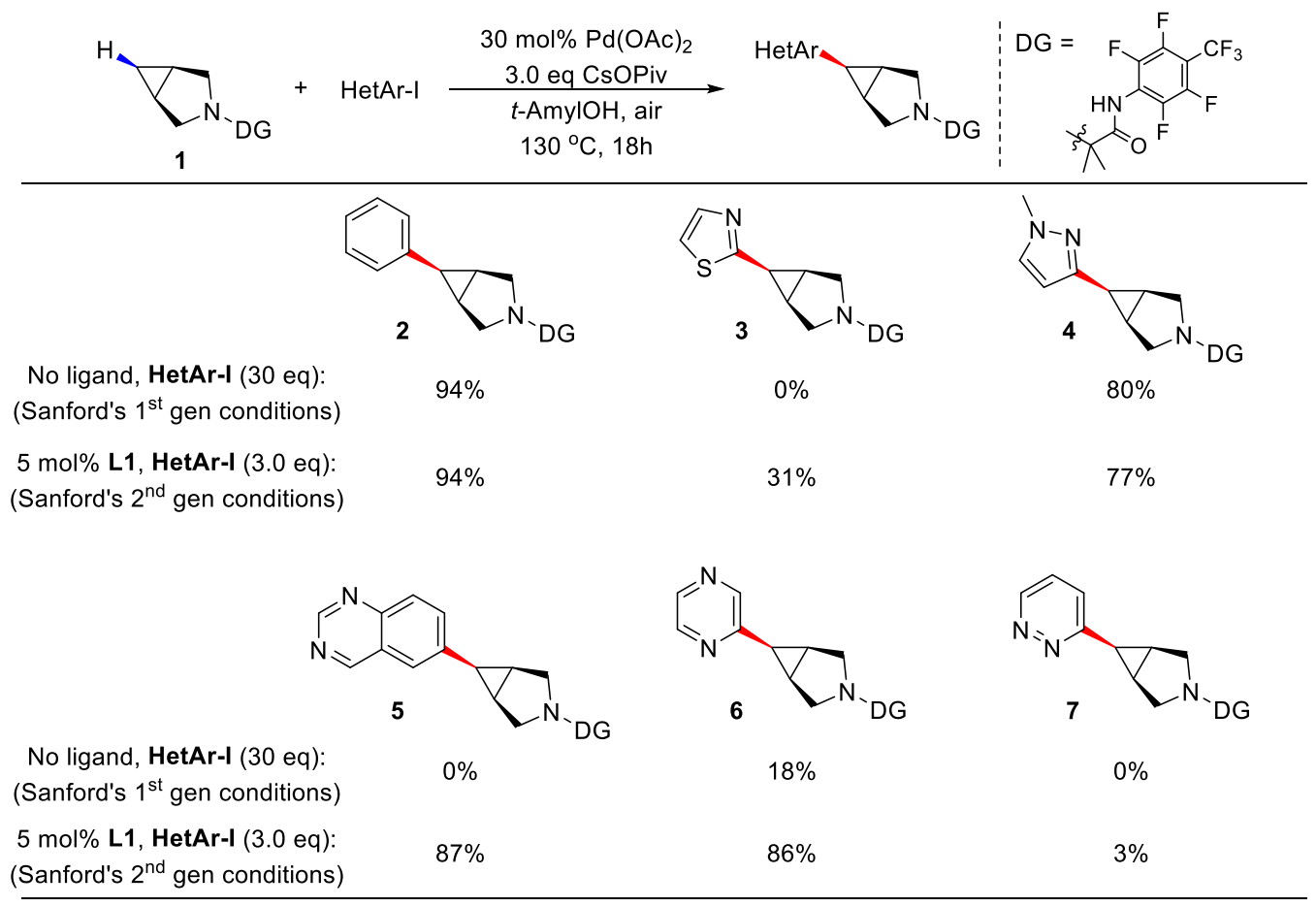

Each reaction was performed in a $1.0 \mathrm{~mL}$ vial for the 96-well Paradox heating plate (https://www.analytical-sales.com/product-category/photoredox-parallel-synthesis/parallel-synthesis-

optimization-plates/parallel-synthesis-96-well/). $\mathrm{Pd}(\mathrm{OAc})_{2}$ was prepared as stock solution in DCM (0.05 M). L1 was prepared as stock solution in DCM (0.02 M). Amine 1 (1.0 eq) and CsOPiv (3.0 eq) were prepared as stock solution in t- $\mathrm{AmylOH}(0.1 \mathrm{M}$ for 1$)$.

To each vial was added $\mathrm{Pd}(\mathrm{OAc})_{2}$ stock solution (60 uL, $30 \mathrm{~mol} \%$ ) and $\mathbf{L} 1$ stock solution (50 uL, $10 \mathrm{~mol} \%)$. The solution in each vial was stirred and heated to $50 \sim 60^{\circ} \mathrm{C}$ for $15 \mathrm{~min}$ to remove the solvent. After cooling down to room temperature, heteroaryl iodide (3.0 or 30.0 eq) was weighed out and transferred into each corresponding vial. Then the stock solution of amine $\mathbf{1}$ and CsOPiv (100 uL) was transferred into each vial. Then reaction block was sealed, stirred and heated to $130^{\circ} \mathrm{C}$ for $18 \mathrm{~h}$. After cooling down to room temperature, to each vial wad added DMSO (300 uL). 
Then the mixture in each vial was transferred into a 2-dr vial and the original reaction vial was rinsed with DMSO (300 uL * 2). The combined solution of each sample in 2-dr vial was transferred to 96-well plate automatically by TECAN and subjected to LC/MS analysis (3.5 min). The integrations of the UV peaks of starting material (SM), desired product (DP), desired side product (DSP) and undesired side product (USP) were measured at $232 \mathrm{~nm}$. The according uncalibrated yield was calculated based on the integration ratio of (DP+DSP) / (SM+DP+DSP+USP).

\section{Examples:}
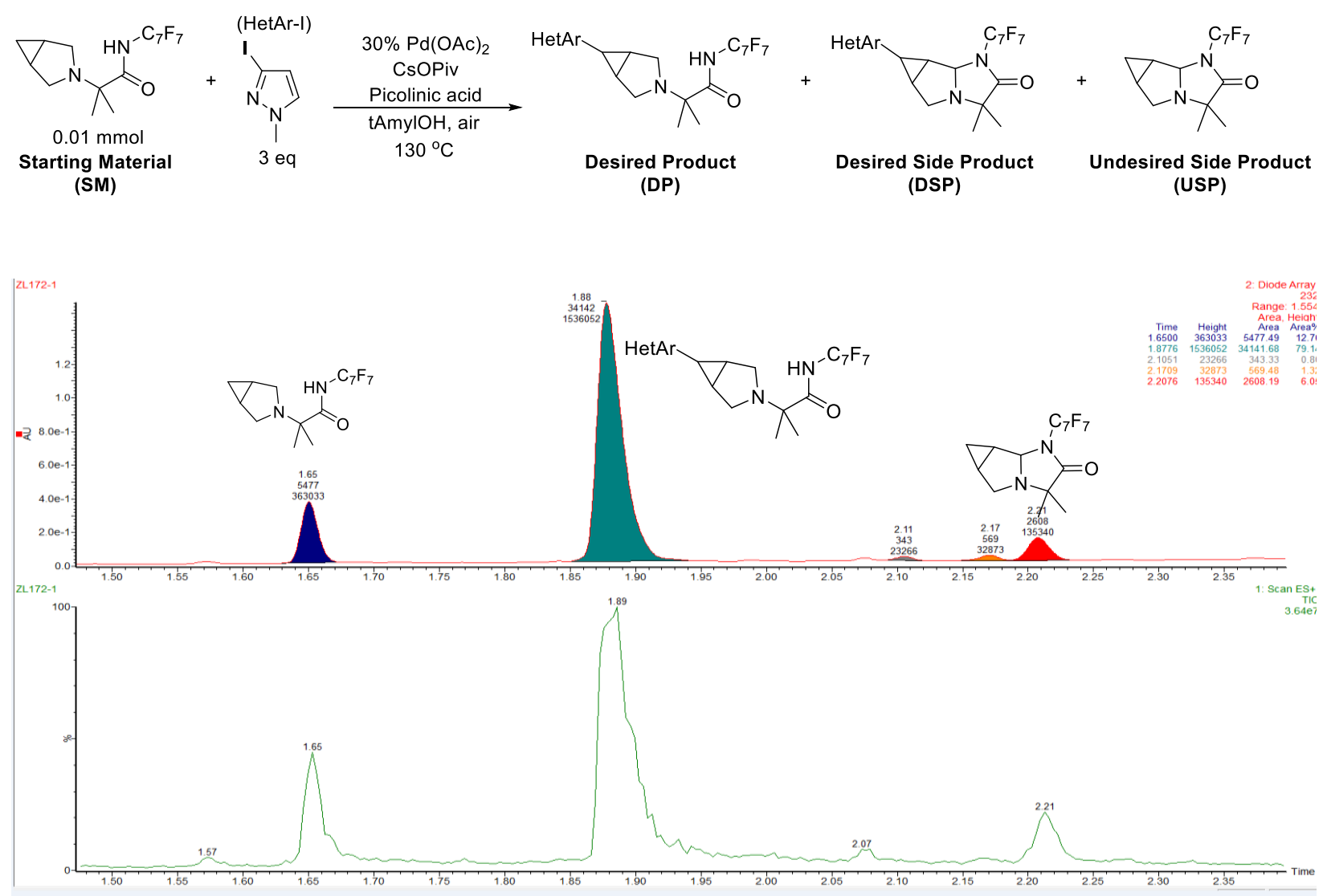

\section{General Procedure for Ligand Screening and Conditions Optimizations.}

Each reaction was performed in a $1.0 \mathrm{~mL}$ vial for the 96 -well Paradox heating plate. $\mathrm{Pd}(\mathrm{OAc})_{2}$ was prepared as stock solution in DCM $(0.05 \mathrm{M})$. Each ligand was prepared as stock solution in DCM (or $\mathrm{MeOH}, 0.02 \mathrm{M}$ ). Amine 1 (1.0 eq) and CsOPiv (3.0 eq) were prepared as stock solution 
in t-AmylOH (0.1M for 1, volatile ligands were also included in this stock solution if necessary). 4CN-4'-Me-biphenyl was employed as internal standard and prepared as stock solution in MeCN (0.1 M).

To each vial was added $\mathrm{Pd}(\mathrm{OAc})_{2}$ stock solution (60 uL, $30 \mathrm{~mol} \%$ ) and the corresponding ligand stock solution (50 uL, $10 \mathrm{~mol} \%$ ). The solution in each vial was stirred and heated to $50 \sim 60{ }^{\circ} \mathrm{C}$ for $15 \mathrm{~min}$ to remove the solvent. After cooling down to room temperature, 2-iodothiazole 8 (3.0 eq) was weighed out and transferred into each vial. Then the stock solution of amine $\mathbf{1}$ and CsOPiv $(100 \mathrm{uL})$ was transferred into each vial. Then reaction block was sealed, stirred and heated to 130 or $150^{\circ} \mathrm{C}$ for $18 \mathrm{~h}$. After cooling down to room temperature, to each vial wad added the stock solution of internal standard (100 uL) and DMSO (200 uL). Then the mixture in each vial was transferred into a 2-dr vial and the original reaction vial was rinsed with DMSO (300 uL * 2). The combined solution of each sample in $2-\mathrm{dr}$ vial was transferred to 96 -well plate automatically by TECAN and subjected to LC/MS analysis. The integrations of UV peaks of desired product (DP), desired side product (DSP) and internal standard (I.S.) were measured at $232 \mathrm{~nm}$. The calibration curve of integration ratio vs. mole ratio between DP and I.S. was performed. In general, DSP was detected $<10 \%$ and we assumed the coefficient of DSP/I.S. was the same as DP/I.S. The calibrated yield of product (DP+DSP) was then calculated based on their integrations of UV peaks at $232 \mathrm{~nm}$.

\section{Examples:}

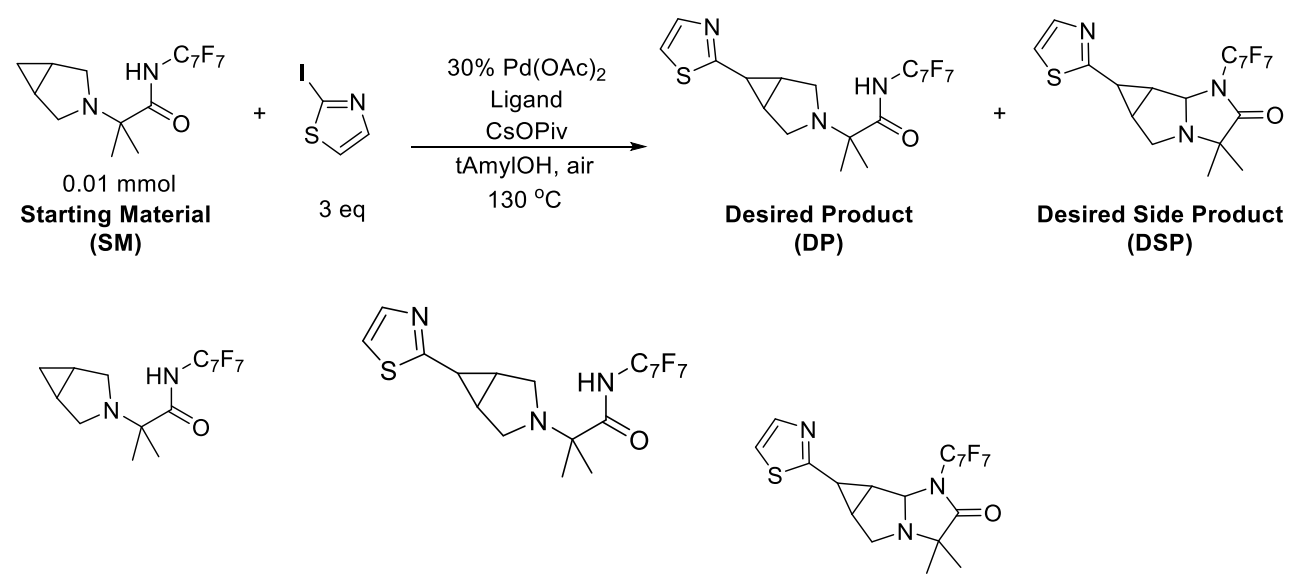




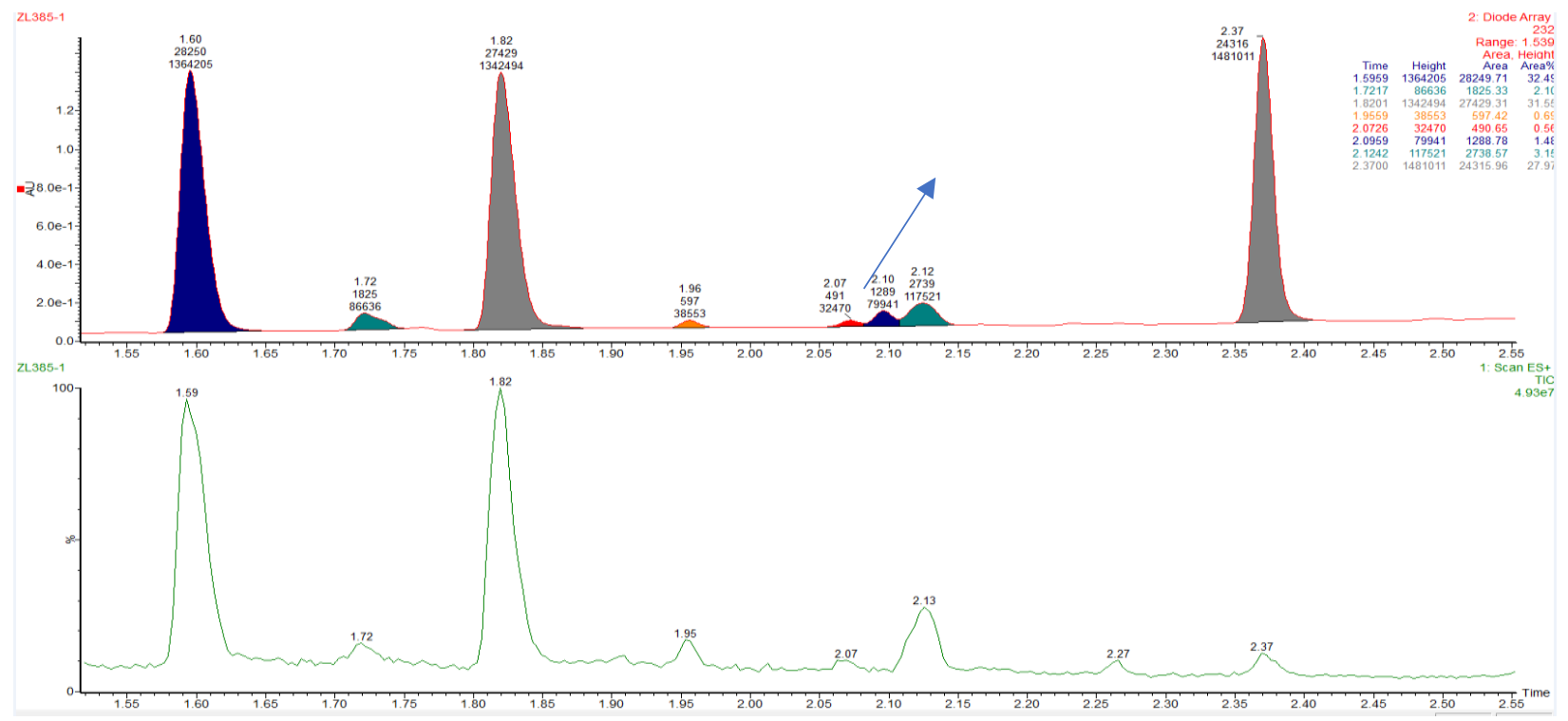

3. Structure of Amine Substrates S-1 and S-2
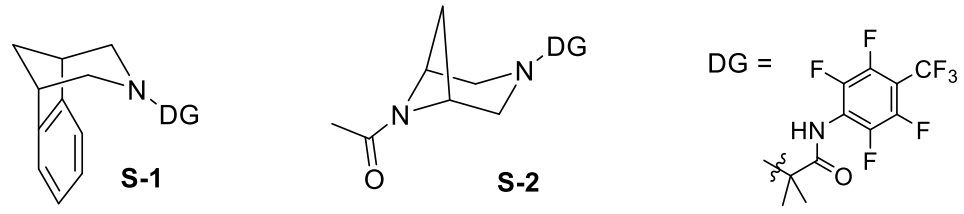

4. Unsuccessful Heteroaryl lodides in the Transannular C-H Heteroarylation Reactions.<smiles>Ic1ncco1</smiles><smiles>Ic1cnoc1</smiles><smiles>Ic1nncs1</smiles><smiles>Cn1cnnc1I</smiles><smiles>Ic1c[nH]cn1</smiles><smiles>Cn1cnc(I)c1</smiles><smiles>Cn1nccc1I</smiles><smiles>Ic1cscn1</smiles><smiles>Ic1cn(Cc2ccccc2)cn1</smiles><smiles>Cn1cncc1I</smiles><smiles>Cn1ccnc1I</smiles><smiles>[TeH3-]n1cnc(I)c1</smiles><smiles>CCn1cnc([Tl])c1</smiles><smiles>Ic1cccnn1</smiles><smiles>Clc1ccc(I)nn1</smiles><smiles>FC(F)(F)c1ccc(I)nn1</smiles><smiles>COc1nnc(Cl)cc1I</smiles> 
$\overbrace{}^{\mathrm{N}}=\overbrace{}^{1}$<smiles>O=c1ncc(I)c[nH]1</smiles><smiles>O=c1ccc(I)c[nH]1</smiles><smiles>Ic1ccc2nc[nH]c2c1</smiles><smiles>Ic1cnc2cc[nH]c2c1</smiles><smiles>Ic1cnc2[nH]ccc2c1</smiles><smiles>Ic1ccc2cn[nH]c2c1</smiles><smiles>Ic1ccc2[nH]ncc2c1</smiles><smiles>Ic1n[nH]c2ccccc12</smiles><smiles>CC(C)(C)OC(=O)n1nc(I)c2ccccc21</smiles><smiles>Fc1ccc2[nH]nc(I)c2c1</smiles><smiles>Fc1cccc2[nH]nc(I)c12</smiles><smiles>FC(F)(F)c1ccc2c(I)n[nH]c2c1</smiles><smiles>Ic1cn(Cc2ccccc2)c2ncccc12</smiles><smiles>CC(C)(C)OC(=O)n1cc(I)c2cccnc21</smiles><smiles>CC(C)(C)OC(=O)n1nc(I)c2cccnc21</smiles><smiles>Fc1ccc2ncc(I)n2c1</smiles><smiles>N#Cc1ccc2ncc(I)n2c1</smiles><smiles>COC(=O)c1ccn2c(I)cnc2c1</smiles><smiles>Brc1cn2c(I)cnc2cn1</smiles><smiles>Clc1ncnc2sc(I)cc12</smiles><smiles>[M]C=[N+]1C(I)=Nc2c(Cl)ncnc21</smiles><smiles>O=C1Cc2ncc(I)cc2N1</smiles><smiles>N#Cc1c[nH]c2ccc(I)cc2c1=O</smiles>

\section{Representative Ligands (112 totals screened).}

Monodentate ligands

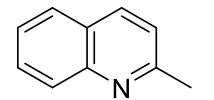

(Jin-Quan Yu, ACIE, 2017)<smiles>FC(F)(F)c1ccncc1C(F)(F)F</smiles>

(Jin-Quan Yu, JACS, 2016)<smiles>O=c1ccc([N+](=O)[O-])c[nH]1</smiles>

(Jin-Quan Yu, JACS, 2018)

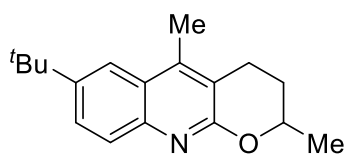

(Jin-Quan Yu, JACS, 2014)
Bidentate ligands<smiles>Cc1cccc(C(=O)O)n1</smiles>

$\mathrm{Ac} \underset{\mathrm{H}}{\mathrm{H}} \sim \mathrm{CO}_{2} \mathrm{H}$

(Jin-Quan Yu, JACS, 2014)

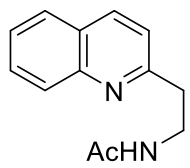

(Jin-Quan Yu, JACS, 2017)

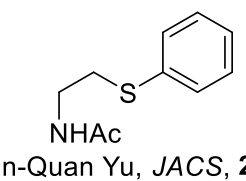

(Jin-Quan Yu, JACS, 2018)

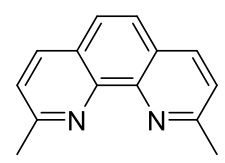

Phosphine ligands in $\operatorname{Pd}(0) / P d(I I)$ catalysis
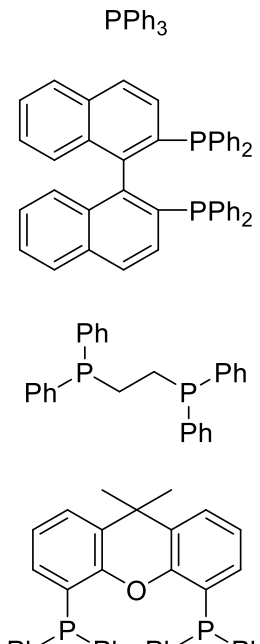

$\mathrm{Ph}^{-\mathrm{P}_{-}} \mathrm{Ph} \mathrm{Ph}^{-\mathrm{P}_{-}}{ }_{\mathrm{Ph}}$

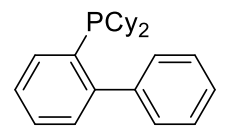

6. ${ }^{1} \mathrm{H},{ }^{19} \mathrm{~F},{ }^{13} \mathrm{C}\left\{{ }^{1} \mathrm{H}\right\}$ NMR Spectra 

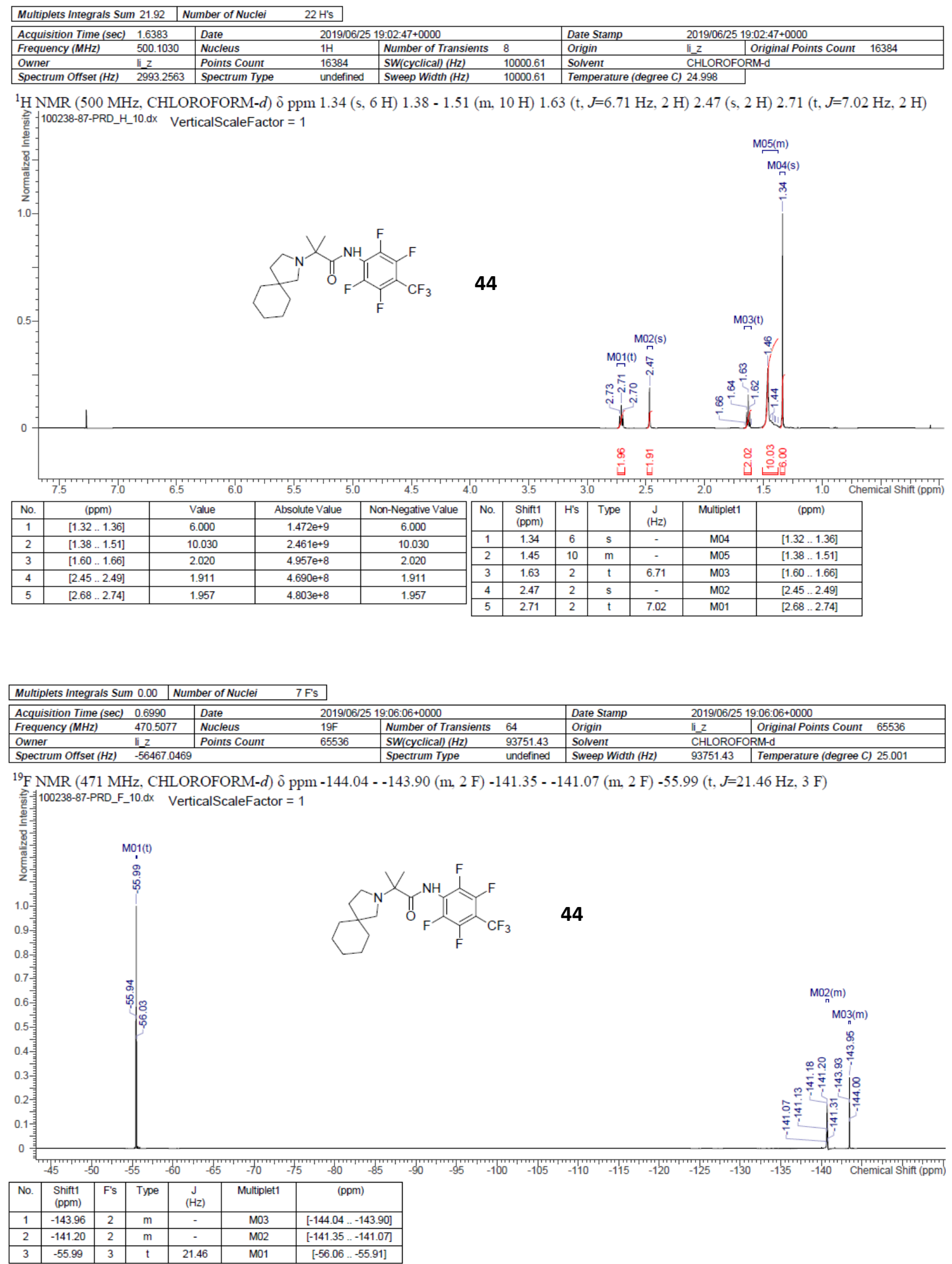


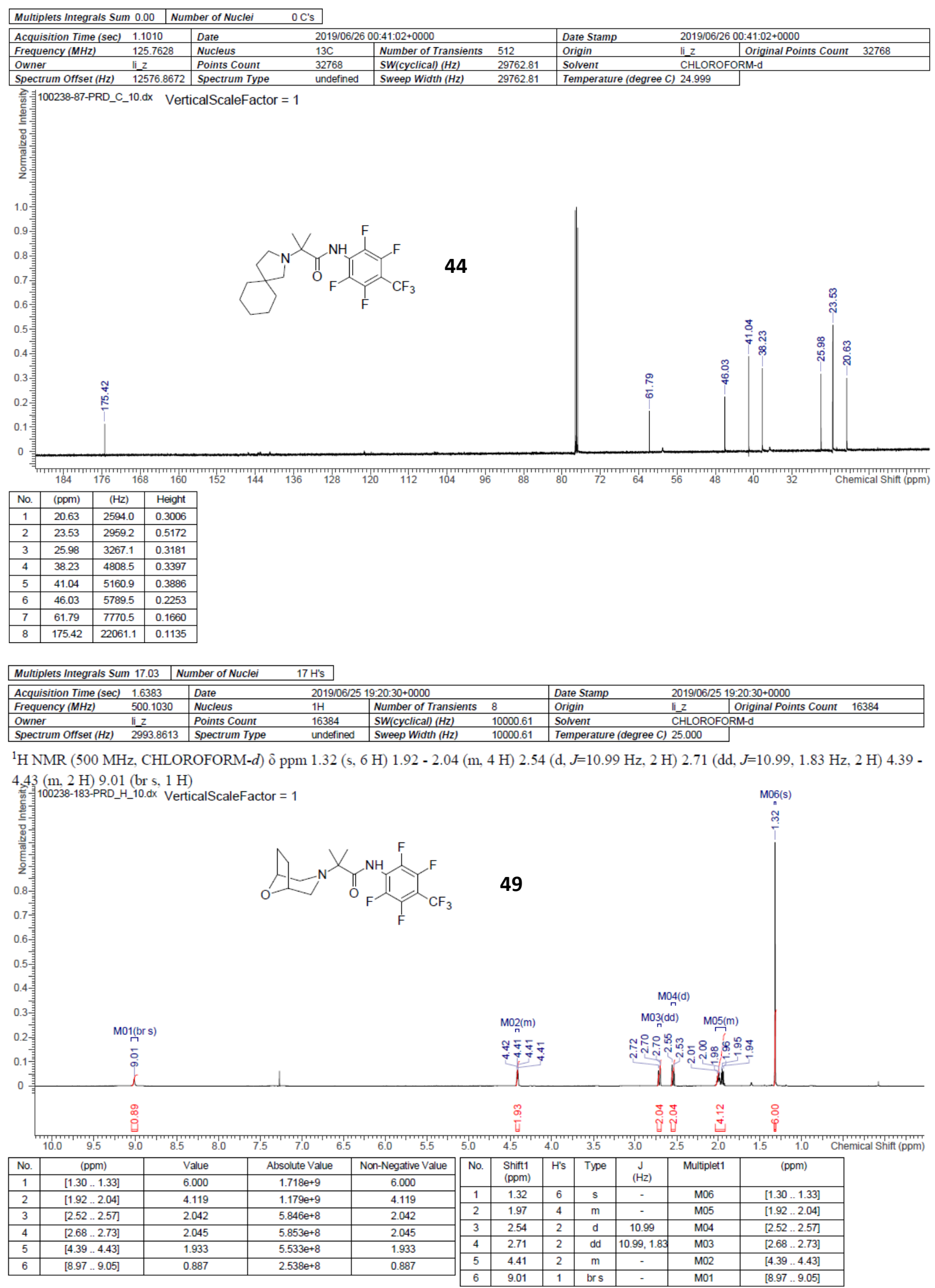




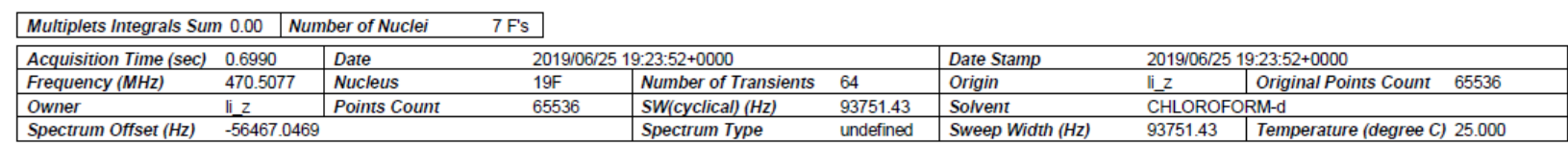

${ }^{19} \mathrm{~F}$ NMR $(471 \mathrm{MHz}$, CHLOROFORM- $d$ ) $\delta$ ppm -143.81 - -143.62 (m. 2 F) -140.97 - -140.70 (m. $2 \mathrm{~F})-56.03(\mathrm{t}, J=21.46 \mathrm{~Hz}, 3 \mathrm{~F})$

总青100238-183-PRD_F_10.dx motetticalScaleFactor = 1

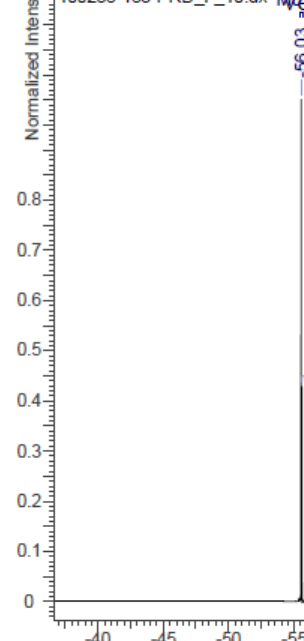

$\stackrel{8}{8}$<smiles>CC(C)(C(=O)Nc1c(F)c(F)c(C(F)(F)F)c(F)c1F)N1CC2CCC(C1)O2</smiles>

49

sis

$\operatorname{M02}(m)$

$\mathrm{M} 03(\mathrm{~m})$

雍

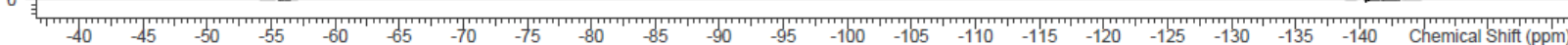

\begin{tabular}{|c|c|c|c|c|c|c|}
\hline No. & $\begin{array}{c}\text { Shift1 } \\
(\mathrm{ppm})\end{array}$ & F's & Type & $\begin{array}{c}\mathrm{J} \\
(\mathrm{Hz})\end{array}$ & Multiplet1 & $(\mathrm{ppm})$ \\
\hline 1 & -143.71 & 2 & $\mathrm{~m}$ & - & $\mathrm{M} 03$ & {$[-143.81 \ldots-143.62]$} \\
\hline 2 & -140.83 & 2 & $\mathrm{~m}$ & - & M02 & {$[-140.97 \ldots-140.70]$} \\
\hline 3 & -56.03 & 3 & $\mathrm{t}$ & 21.46 & M01 & {$[-56.11 \ldots-55.96]$} \\
\hline
\end{tabular}

\begin{tabular}{|l|l|l|}
\hline Multiplets Integrals Sum 0.00 & Number of Nuclei & 0 C's \\
\hline
\end{tabular}

\begin{tabular}{|ll|ll|ll|l|l|l|}
\hline Acquisition Time $(\mathrm{sec})$ & 1.1010 & Date & $2019 / 06 / 26$ & $01: 27: 59+0000$ & Date Stamp & $2019 / 06 / 2601: 27: 59+0000$ \\
\hline Frequency $(\mathrm{MHz})$ & 125.7628 & Nucleus & $13 \mathrm{C}$ & Number of Transients & 512 & Origin & li_z & Original Points Count \\
\hline Owner & li_z & Points Count & 32768 & SW/cyclical) $(\mathrm{Hz})$ & 29762.81 & Solvent & CHLOROFORM-d \\
\hline Spectrum Offset $(\mathrm{Hz})$ & 12576.8672 & Spectrum Type & undefined & Sweep Width $(\mathrm{Hz})$ & 29762.81 & Temperature (degree C) 25.001 & \\
\hline
\end{tabular}

\begin{tabular}{|ll|l|l}
\hline Spectrum Offset $(\mathrm{Hz})$ & 12576.8672 & Spectrum Type \\
\hline
\end{tabular}

空责100238-183-PRD_C_10.dx VerticalScaleFactor $=1$

등글

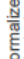

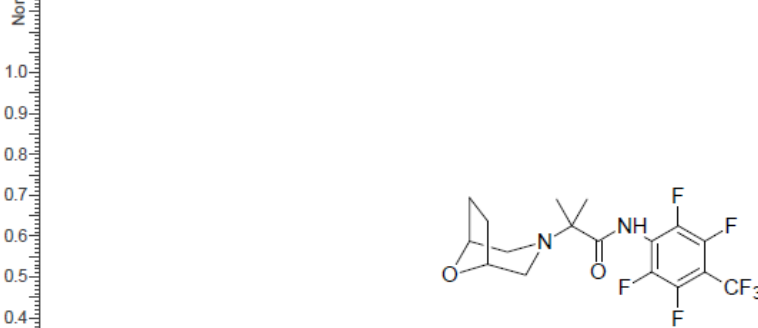

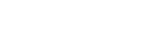

0.3 告

0.1 贯

\begin{tabular}{|c|c|c|c|}
\multicolumn{5}{|c|}{176} & 168 & & 160 \\
\hline No. & $(\mathrm{ppm})$ & $(\mathrm{Hz})$ & Height \\
\hline 1 & 20.47 & 2574.0 & 0.8292 \\
\hline 2 & 28.09 & 3532.3 & 0.3996 \\
\hline 3 & 51.89 & 6526.1 & 0.5549 \\
\hline 4 & 64.10 & 8061.2 & 0.1512 \\
\hline 5 & 74.43 & 9361.0 & 1.0000 \\
\hline 6 & 174.91 & 21996.6 & 0.1357 \\
\hline
\end{tabular}




\begin{tabular}{|c|c|c|c|c|c|c|c|c|c|}
\hline Multiplets Integrals Sum & 19.24 & Number of Nuclei & $19 \mathrm{H}$ 's & & & & & & \\
\hline Acquisition Time (sec) & 1.6383 & Date & $2019 / 06 / 25$ & $8: 54: 16+0000$ & & Date Stamp & $2019 / 06$ & $18: 54: 16+0000$ & \\
\hline Frequency $(\mathrm{MHz})$ & 500.1030 & Nucleus & $1 \mathrm{H}$ & Number of Transients & 8 & Origin & li_z & Original Points Count & 16384 \\
\hline Owner & li_z & Points Count & 16384 & SW(cyclical) (Hz) & 10000.61 & Solvent & CHLOR & RM-d & \\
\hline Spectrum Offset $(\mathrm{Hz})$ & 2993.8916 & Spectrum Type & undefined & Sweep Width $(\mathrm{Hz})$ & 10000.61 & Temperature (degree $C$ ) & 24.999 & & \\
\hline
\end{tabular}

${ }^{1} \mathrm{H}$ NMR $(500 \mathrm{MHz}$, CHLOROFORM- $d$ ) $\delta \mathrm{ppm} 1.27$ (t, $J=7.33 \mathrm{~Hz}, 3 \mathrm{H}) 1.31(\mathrm{t}, J=4.58 \mathrm{~Hz}, 1 \mathrm{H}) 1.34$ (s, $\left.3 \mathrm{H}\right) 1.39$ (s, $\left.3 \mathrm{H}\right) 1.47$ (dd, $J=8.55,4.27$ $\mathrm{Hz}, 1 \mathrm{H}) 2.00-2.06(\mathrm{~m}, 1 \mathrm{H}) 2.81(\mathrm{dd}, J=9.16,3.66 \mathrm{~Hz}, 1 \mathrm{H}) 2.91(\mathrm{~d}, J=9.16 \mathrm{~Hz}, 1 \mathrm{H}) 3.04(\mathrm{~d}, J=9.16 \mathrm{~Hz}, 1 \mathrm{H}) 3.10(\mathrm{~d}, J=9.16 \mathrm{~Hz}, 1 \mathrm{H}) 4.17$ (q, $J=7.12 \mathrm{~Hz}, 2 \mathrm{H}) 8.82$ (br s, $1 \mathrm{H}$ )

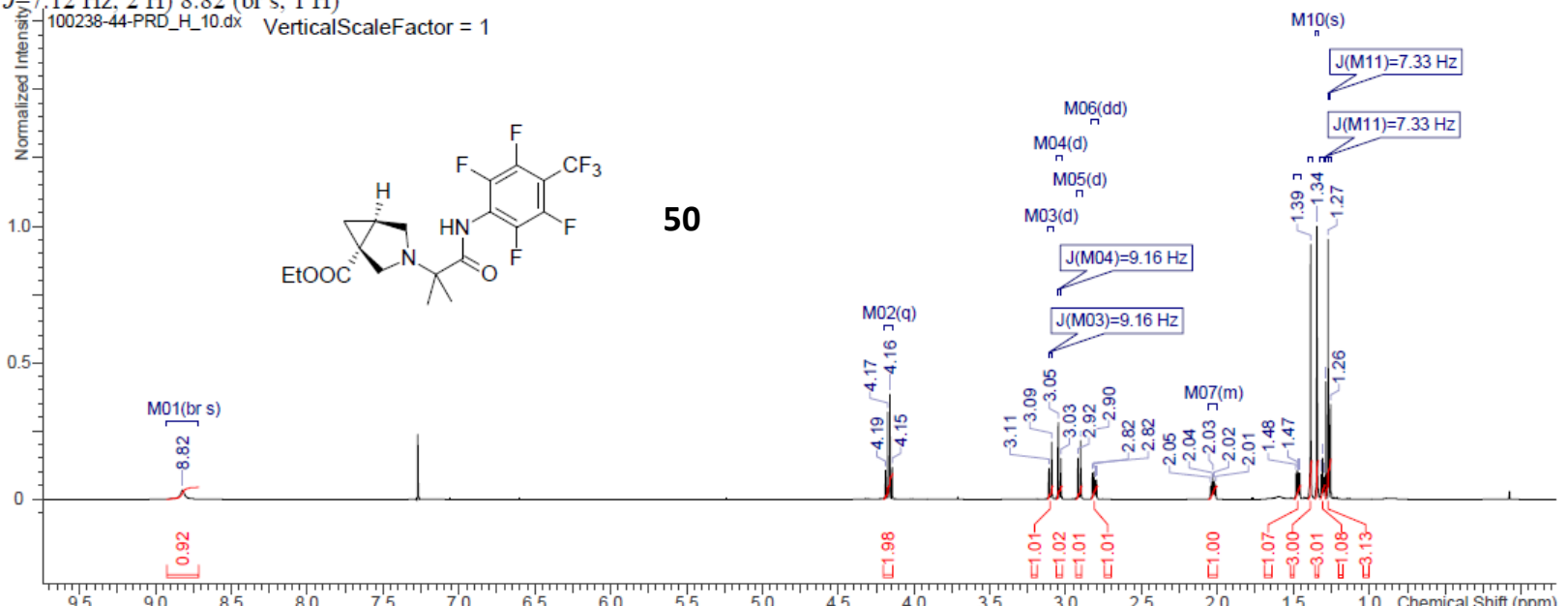

\begin{tabular}{|c|c|c|c|c|c|c|c|c|c|c|c|c|c|}
\hline No. & $\begin{array}{l}\text { Shift1 } \\
\text { (ppm) }\end{array}$ & H's & Type & $\begin{array}{c}\mathrm{J} \\
(\mathrm{Hz})\end{array}$ & Multiplet1 & (ppm) & No. & $\begin{array}{l}\text { Shift1 } \\
(\mathrm{ppm})\end{array}$ & H's & Type & $\begin{array}{c}\mathrm{J} \\
(\mathrm{Hz})\end{array}$ & Multiplet1 & (ppm) \\
\hline 1 & 1.27 & 3 & $\mathrm{t}$ & 7.33 & M11 & {$\left[\begin{array}{llll}1.25 & \ldots & 1.29\end{array}\right]$} & 7 & 2.81 & 1 & dd & $9.16,3.66$ & M06 & {$[2.79 \ldots 2.83]$} \\
\hline 2 & 1.31 & 1 & $t$ & 4.58 & M12 & {$[1.29 \ldots 1.32]$} & 8 & 2.91 & 1 & $d$ & 9.16 & M05 & {$\left[\begin{array}{llll}2.89 & 2.93]\end{array}\right.$} \\
\hline 3 & 1.34 & 3 & $\mathrm{~s}$ & - & M10 & {$[1.33 \ldots 1.36]$} & 9 & 3.04 & 1 & d & 9.16 & M04 & {$[3.02 \ldots 3.06]$} \\
\hline 4 & 1.39 & 3 & $\mathrm{~s}$ & - & M09 & {$[1.38 \ldots 1.40]$} & 10 & 3.10 & 1 & d & 9.16 & M03 & {$\left[\begin{array}{lll}3.08 & . .3 & 3.12\end{array}\right]$} \\
\hline 5 & 1.47 & 1 & dd & $8.55,4.27$ & M08 & {$[1.45 \ldots 1.49]$} & 11 & 4.17 & 2 & $q$ & 7.12 & M02 & {$[4.14 \ldots 4.20]$} \\
\hline 6 & 2.03 & 1 & $\mathrm{~m}$ & - & M07 & {$[2.00 \ldots 2.06]$} & 12 & 8.82 & 1 & $\mathrm{brs}$ & - & M01 & {$\left[\begin{array}{l}8.72 \ldots \\
{[.8 .93]}\end{array}\right.$} \\
\hline
\end{tabular}

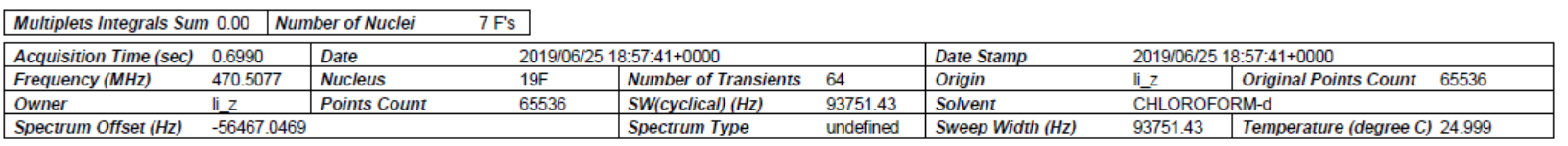

${ }^{19} \mathrm{~F}$ NMR (471 MHz, CHLOROFORM- $d$ ) $\delta$ ppm $-144.25--143.97$ (m, 2 F) $-141.05--140.72(\mathrm{~m}, 2 \mathrm{~F})-56.00(\mathrm{t}, J=21.46 \mathrm{~Hz}, 3 \mathrm{~F})$

兽青100238-44-PRD_F_10.dx MertticalScaleFactor $=1$

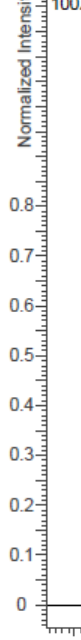

\begin{tabular}{|c|c|c|c|c|c|c|}
\hline No. & $\begin{array}{c}\text { Shift1 } \\
(\mathrm{ppm})\end{array}$ & $\mathrm{F}$ 's & Type & $\begin{array}{c}\mathrm{J} \\
(\mathrm{Hz})\end{array}$ & Multiplet1 & $(\mathrm{ppm})$ \\
\hline 1 & -144.06 & 2 & $\mathrm{~m}$ & - & $\mathrm{M} 03$ & {$[-144.25 \ldots-143.97]$} \\
\hline 2 & -140.90 & 2 & $\mathrm{~m}$ & - & M02 & {$[-141.05 \ldots-140.72]$} \\
\hline 3 & -56.00 & 3 & $\mathrm{t}$ & 21.46 & M01 & {$[-56.11 \ldots-55.92]$} \\
\hline
\end{tabular}




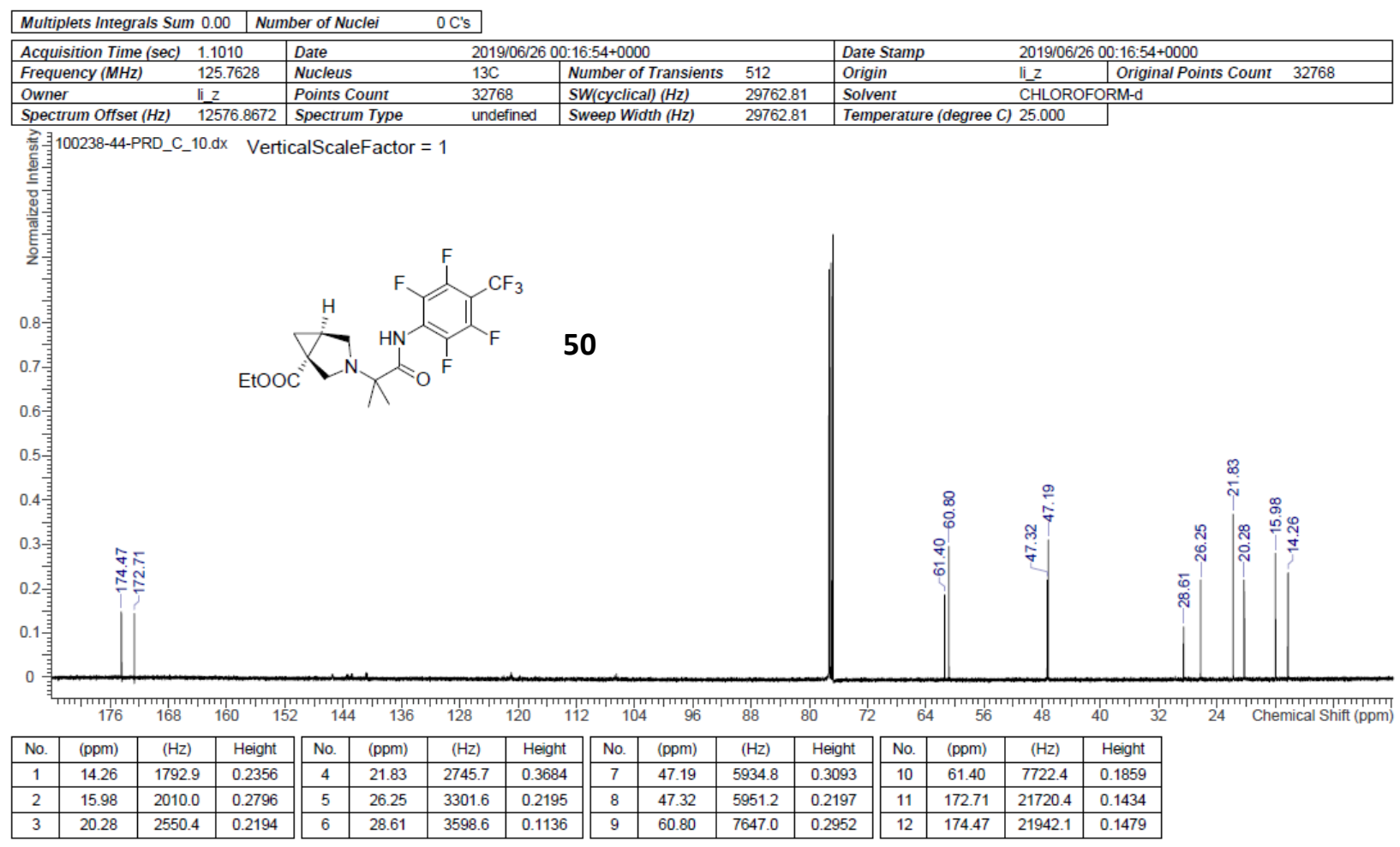

\begin{tabular}{|c|c|c|c|c|c|c|c|c|c|}
\hline Multiplets Integrals Sum & 17.52 & Number of Nuclei & $18 \mathrm{H}$ 's & & & & & & \\
\hline Acquisition Time (sec) & 1.6383 & Date & $2019 / 06 / 25$ & $8: 45: 34+0000$ & & Date Stamp & $2019 / 06 / 2$ & $8: 45: 34+0000$ & \\
\hline Frequency $(\mathrm{MHz})$ & 500.1030 & Nucleus & $1 \mathrm{H}$ & Number of Transients & 8 & Origin & li_z & Original Points Count & 16384 \\
\hline Owner & li_z & Points Count & 16384 & SW(cyclical) $(\mathrm{Hz})$ & 10000.61 & Solvent & CHLORO & RM-d & \\
\hline Spectrum Offset $(\mathrm{Hz})$ & 2993.2659 & Spectrum Type & undefined & Sweep Width $(\mathrm{Hz})$ & 10000.61 & Temperature (degree $\mathrm{C}$ ) & 25.000 & & \\
\hline
\end{tabular}

${ }^{1} \mathrm{H}$ NMR $(500 \mathrm{MHz}$, CHLOROFORM- $d$ ) $\delta \mathrm{ppm} 1.38(\mathrm{~s}, 3 \mathrm{H}) 1.45(\mathrm{~s}, 3 \mathrm{H}) 1.55(\mathrm{~d}, J=8.55 \mathrm{~Hz}, 1 \mathrm{H}) 1.98(\mathrm{~s}, 3 \mathrm{H}) 2.62-2.70(\mathrm{~m}, 1 \mathrm{H}) 2.79(\mathrm{~d}$, $J=10.99 \mathrm{~Hz}, 1 \mathrm{H}) 2.87(\mathrm{~d}, J=10.99 \mathrm{~Hz}, 1 \mathrm{H}) 3.30(\mathrm{br} \mathrm{d}, J=9.77 \mathrm{~Hz}, 1 \mathrm{H}) 3.42($ br d, $J=9.77 \mathrm{~Hz}, 1 \mathrm{H}) 4.35$ (br d, $J=3.66 \mathrm{~Hz}, 2 \mathrm{H}) 8.64$ (br s, $1 \mathrm{H})$

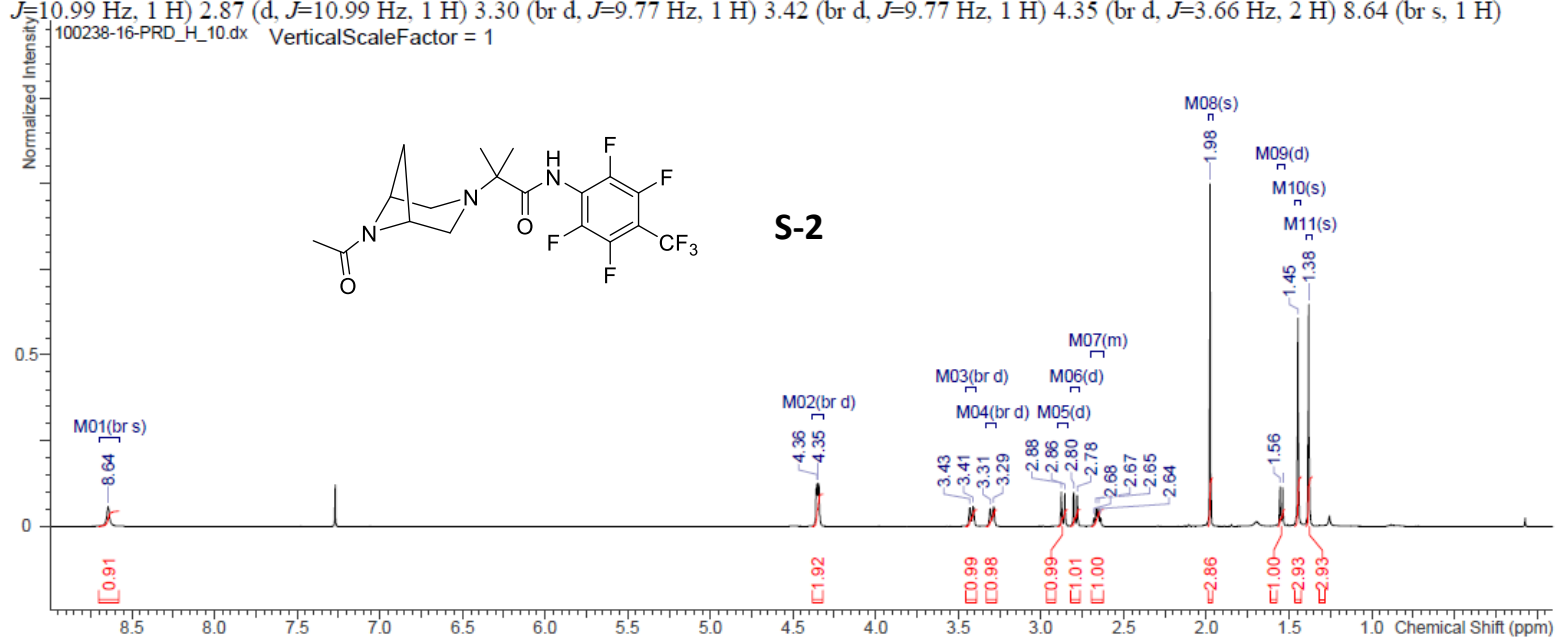

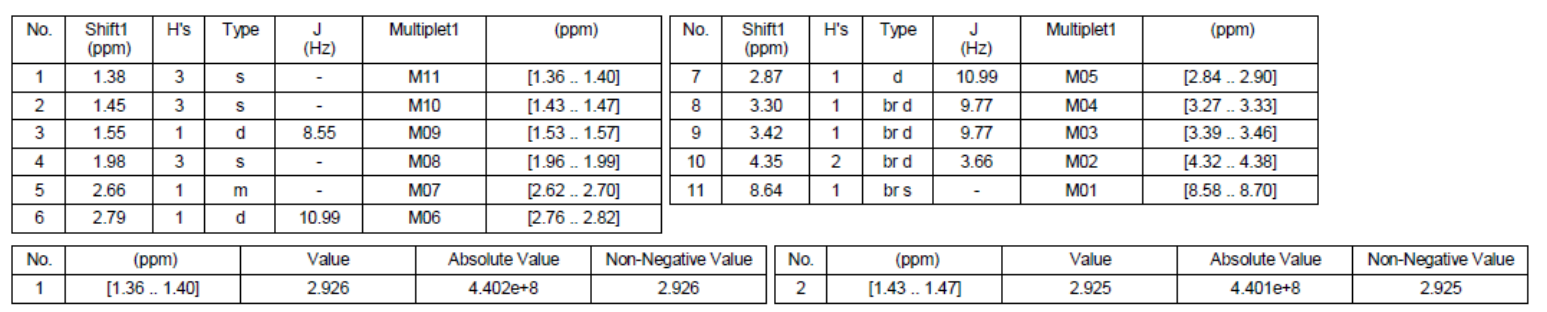



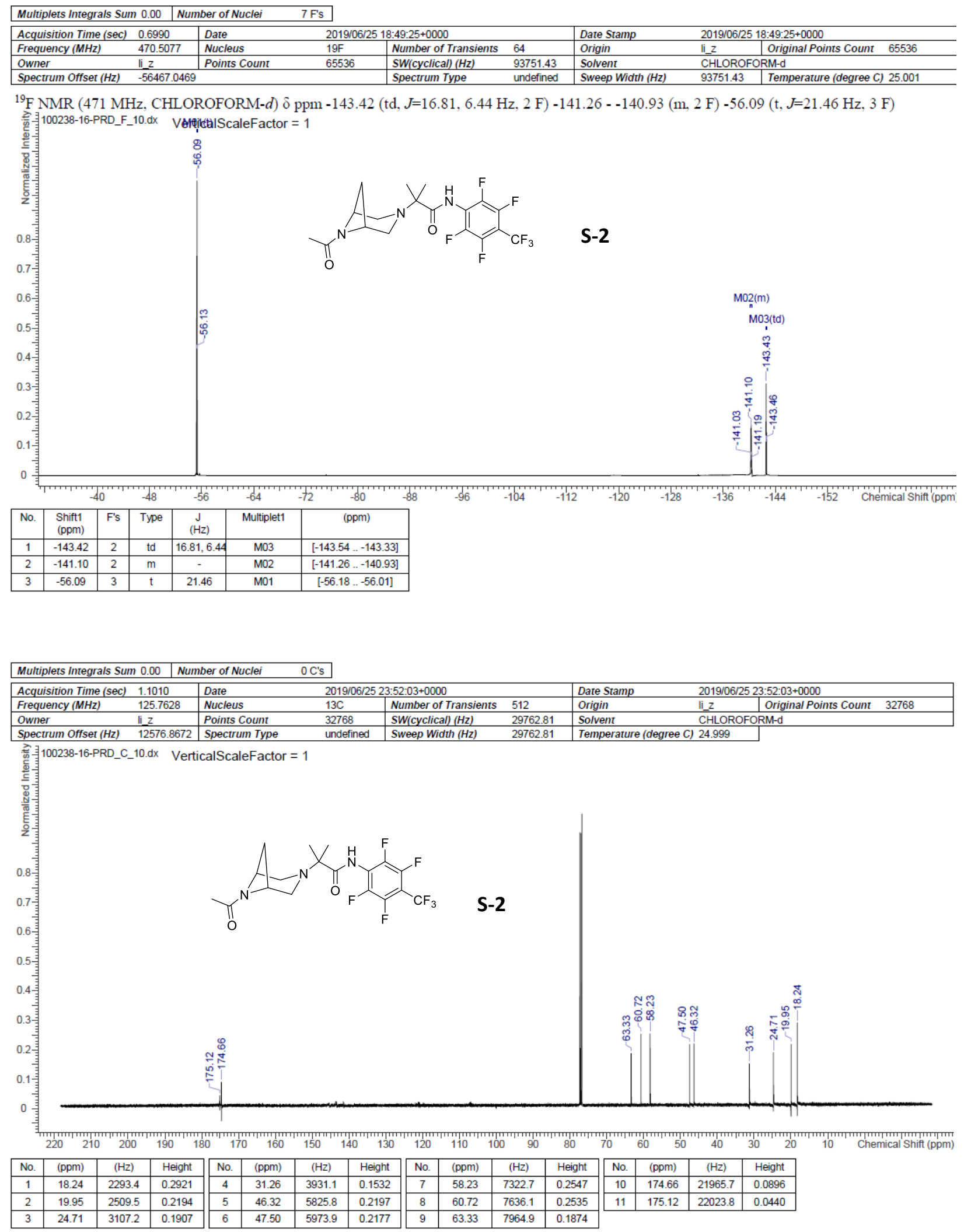


\begin{tabular}{|ll|ll|ll|l|l|l|}
\hline Acquisition Time $(\mathrm{sec})$ & 1.6383 & Date & $2019 / 05 / 16$ & $13: 16: 04+0000$ & & Date Stamp & $2019 / 05 / 1613: 16: 04+0000$ \\
\hline Frequency $(\mathrm{MHz})$ & 500.1030 & Nucleus & $1 \mathrm{H}$ & Number of Transients & 8 & Origin & li_z \\
\hline Owner & li z & Points Count & 16384 & SW $($ cyclical) $(\mathrm{Hz})$ & 10000.61 & Solvent & CHLOROFORM-d \\
\hline Spectrum Offset $(\mathrm{Hz})$ & 2988.8701 & Spectrum Type & undefined & Sweep Width $(\mathrm{Hz})$ & 10000.61 & Temperature $($ degree $\mathrm{C})$ & 24.999 \\
\hline
\end{tabular}

${ }^{1} \mathrm{H}$ NMR $(500 \mathrm{MHz}$, CHLOROFORM-d) $\delta \mathrm{ppm} 1.17$ (s, 6 H) $2.03-2.09$ (m, $2 \mathrm{H}) 2.20$ (t, J=7.33 Hz, $\left.1 \mathrm{H}\right) 2.86-2.95$ (m, $\left.2 \mathrm{H}\right) 3.17$ (d, J=9.77 Hz, $2 \mathrm{H}) 7.17$ (d, $J=3.05 \mathrm{~Hz}, 1 \mathrm{H}) 7.39$ (d, $J=3.05 \mathrm{~Hz}, 1 \mathrm{H}) 7.55$ (br s, $1 \mathrm{H})$

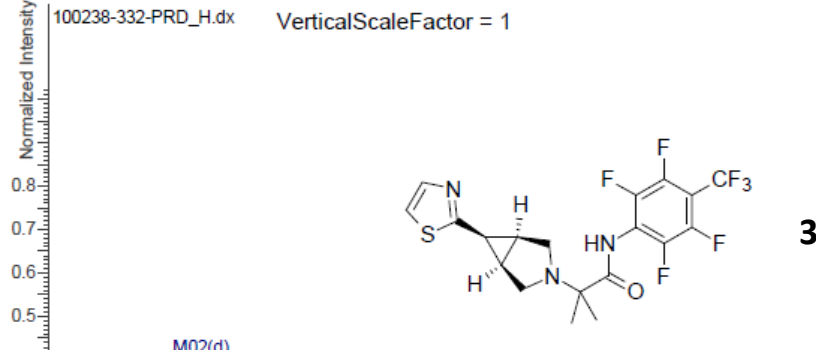

3

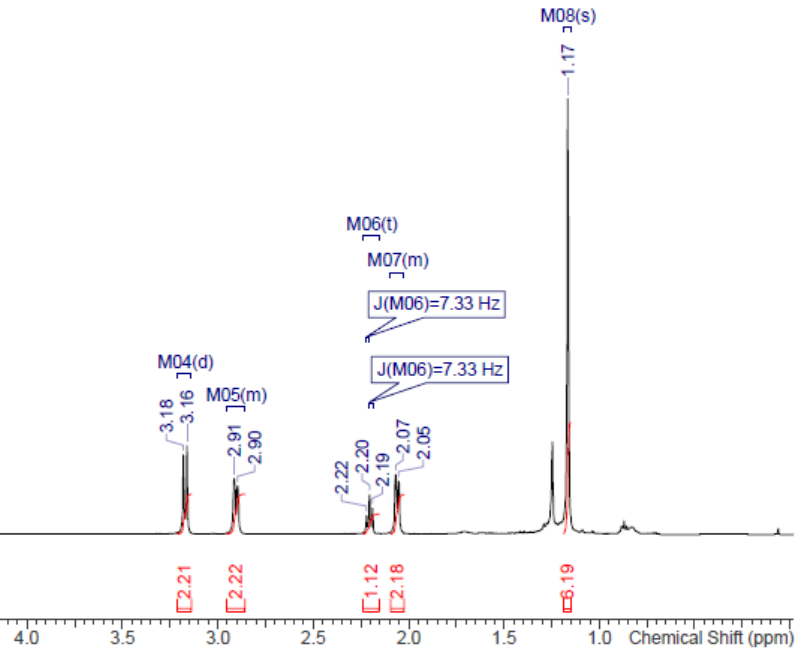

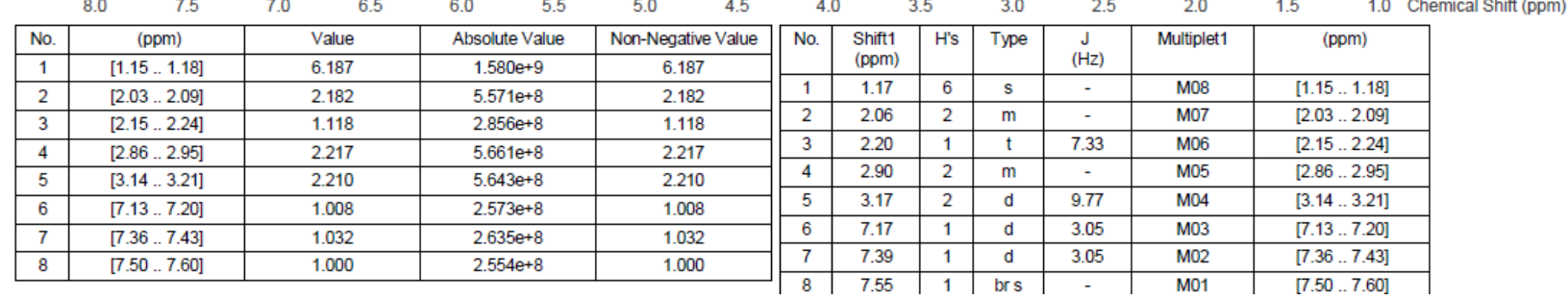

6/2/2019 3:07:07 PM

\begin{tabular}{|l|l|}
\hline Multiplets Integrals Sum 0.00 & Number of Nuclei 7 F's \\
\hline
\end{tabular}

\begin{tabular}{|c|c|c|c|c|c|c|c|c|c|}
\hline Acquisition Time (sec) & 0.6990 & Date & \multicolumn{3}{|c|}{$2019 / 05 / 16$ 13:21:18+0000 } & Date Stamp & \multicolumn{3}{|c|}{$2019 / 05 / 16$ 13:21:18+0000 } \\
\hline Frequency $(\mathrm{MHz})$ & 470.5077 & Nucleus & $19 \mathrm{~F}$ & Number of Transients & 64 & Origin & li_z & Original Points Count & 65536 \\
\hline Owner & li_z & Points Count & 65536 & SW(cyclical) $(\mathrm{Hz})$ & 93751.43 & Solvent & \multicolumn{3}{|c|}{ CHLOROFORM-d } \\
\hline Spectrum Offset $(\mathrm{Hz})$ & -56467.046 & & & Spectrum Type & undefined & Sweep Width $(\mathrm{Hz})$ & 93751.43 & Temperature (degree $C$ & 25.000 \\
\hline
\end{tabular}

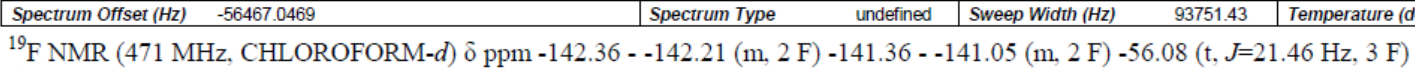

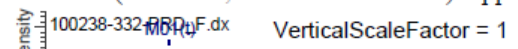

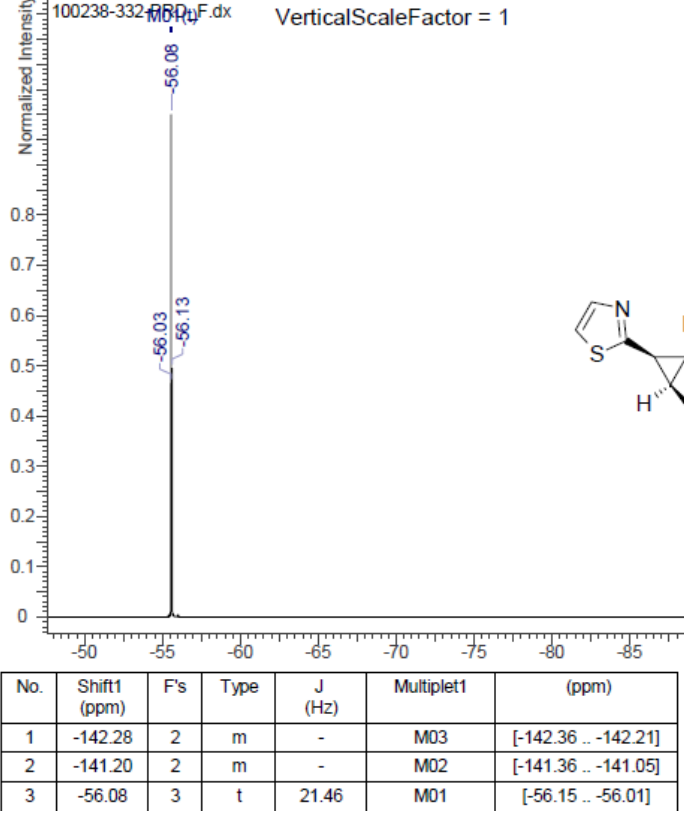




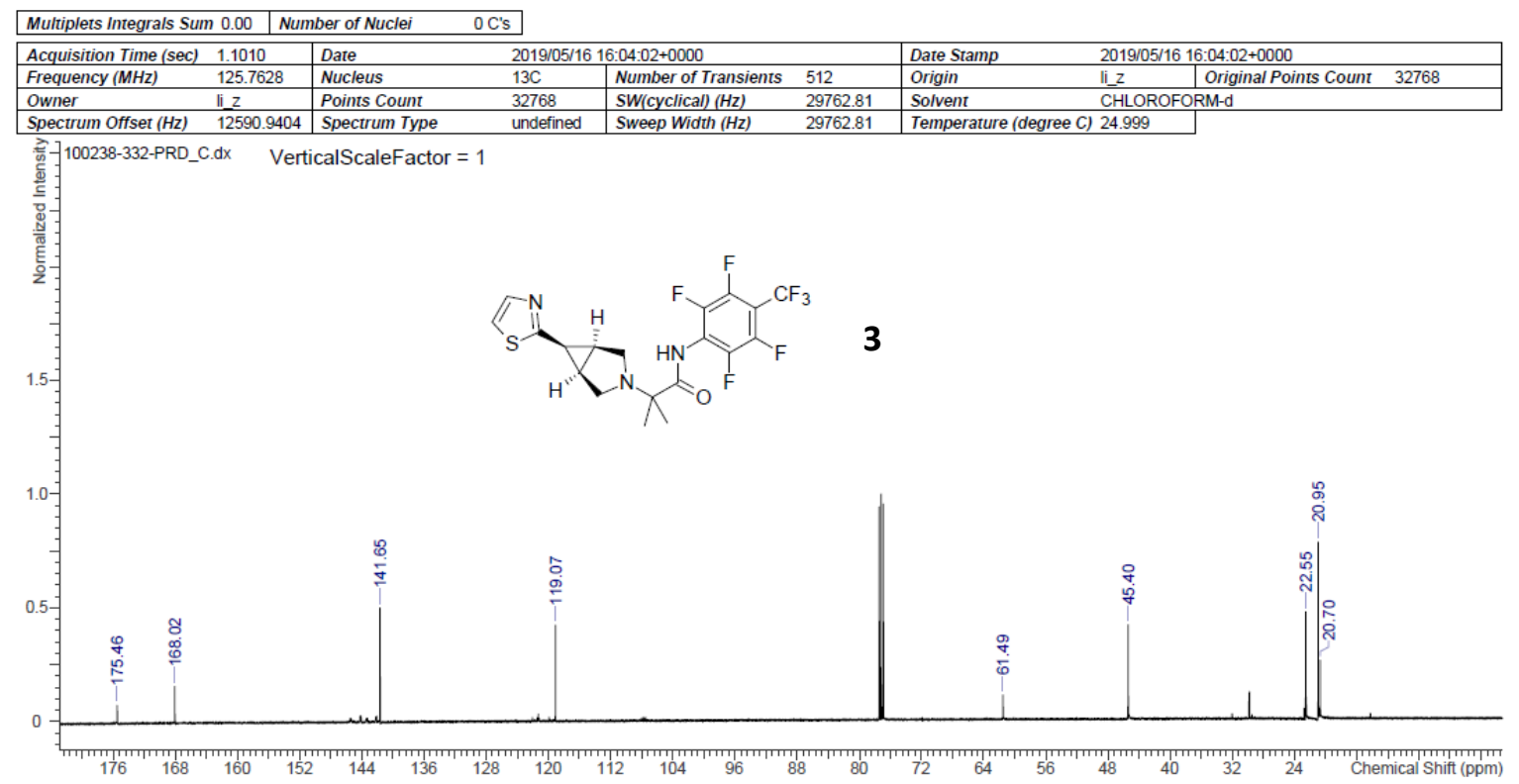

\begin{tabular}{|c|c|c|c|}
\hline No. & $(\mathrm{ppm})$ & $(\mathrm{Hz})$ & Height \\
\hline 1 & 20.70 & 2603.5 & 0.2687 \\
\hline 2 & 20.95 & 2635.3 & 0.7889 \\
\hline 3 & 22.55 & 2836.1 & 0.4826 \\
\hline 4 & 45.40 & 5709.1 & 0.4267 \\
\hline 5 & 61.49 & 7732.8 & 0.1170 \\
\hline 6 & 119.07 & 14974.8 & 0.4244 \\
\hline 7 & 141.65 & 17814.2 & 0.4997 \\
\hline 8 & 168.02 & 21130.5 & 0.1550 \\
\hline 9 & 175.46 & 22067.0 & 0.0708 \\
\hline
\end{tabular}

5/31/2019 11:07-55 AM

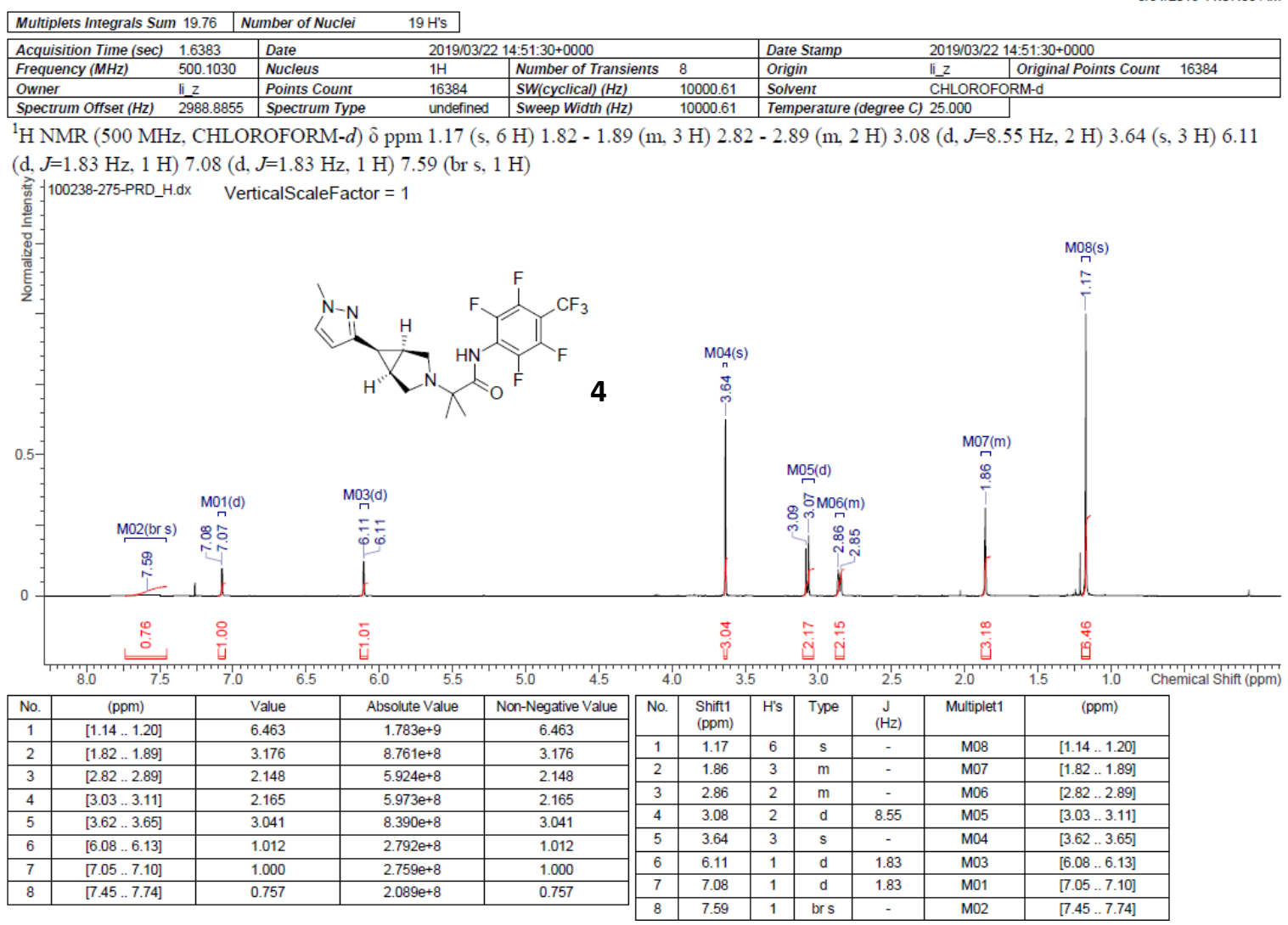


5/31/2019 11:13:22 AM

\begin{tabular}{|l|ll|}
\hline Multiplets Integrals Sum 0.00 & Number of Nuclei $7 \mathrm{Fs}$ \\
\hline
\end{tabular}

\begin{tabular}{|c|c|c|c|c|c|c|c|c|}
\hline Acquisition Time (sec) & 0.6990 & Date & \multicolumn{3}{|c|}{$2019 / 03 / 2214: 57: 54+0000$} & \begin{tabular}{|l|} 
Date Stamp \\
\end{tabular} & \multicolumn{2}{|c|}{$2019 / 03 / 2214: 57: 54+0000$} \\
\hline Frequency $(\mathrm{MHz})$ & 470.5077 & Nucleus & $19 \mathrm{~F}$ & Number of Transients & 64 & Origin & li_z & Original Points Count 65536 \\
\hline Owner & li_z & Points Count & 65536 & SW(cyclical) $(\mathrm{Hz})$ & 93751.43 & Solvent & \multicolumn{2}{|c|}{ CHLOROFORM-d } \\
\hline Spectrum Offset $(\mathrm{Hz})$ & -56467.0469 & & & Spectrum Type & undefined & Sweep Width $(\mathrm{Hz})$ & 93751.43 & Temperature (degree C) 24.999 \\
\hline
\end{tabular}

${ }^{19} \mathrm{~F}$ NMR (471 MHz, CHLOROFORM- $d$ ) $\delta \mathrm{ppm}-142.88$ - -142.66 (m, 2 F) -141.35 - -141.05 (m, 2 F) -56.07 (t, J=21.46 Hz, 3 F)

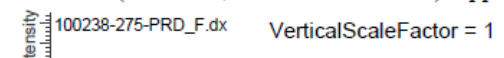

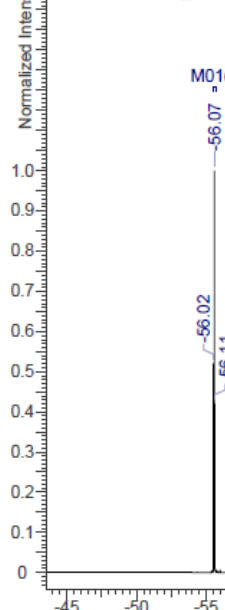

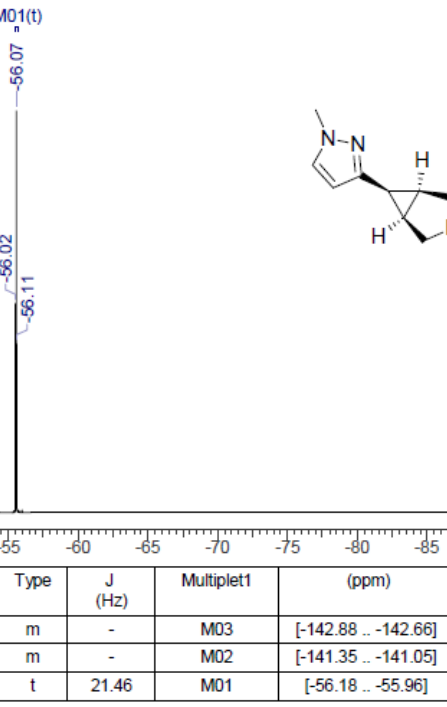

5/31/2019 11:16:52 AM

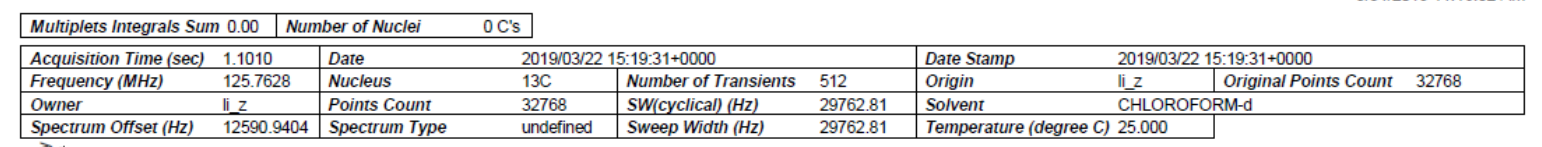

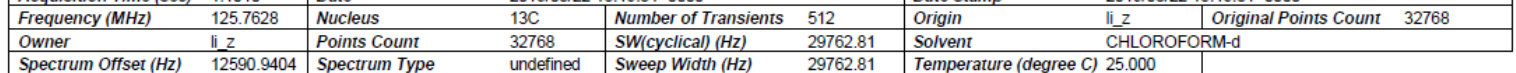

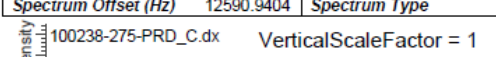

垔起

兽贯

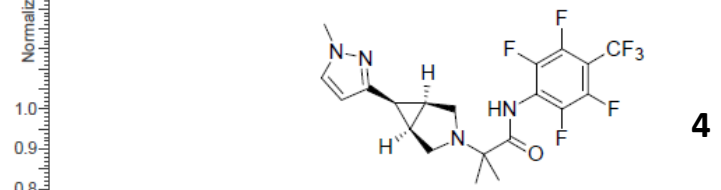

0.8 贯

0.7 旁
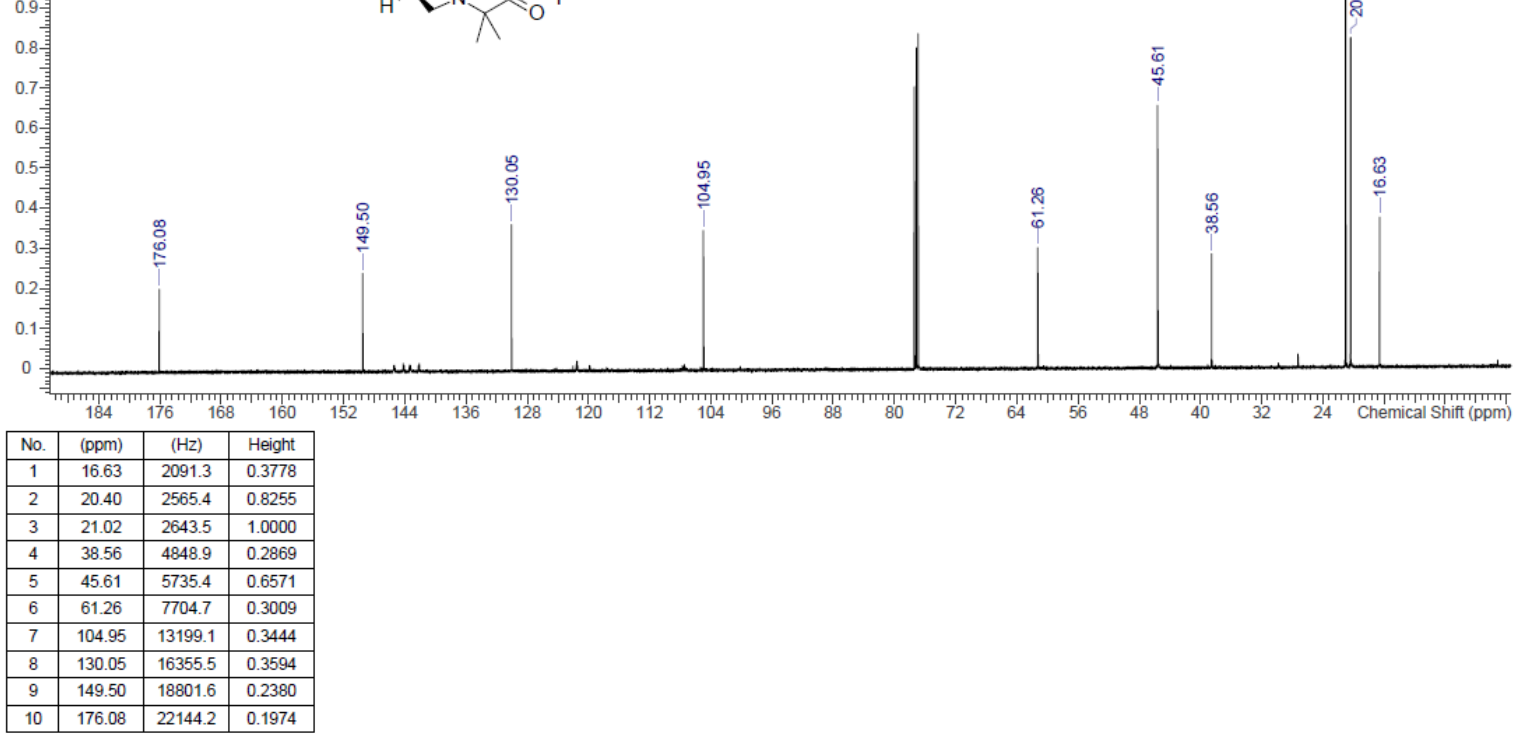


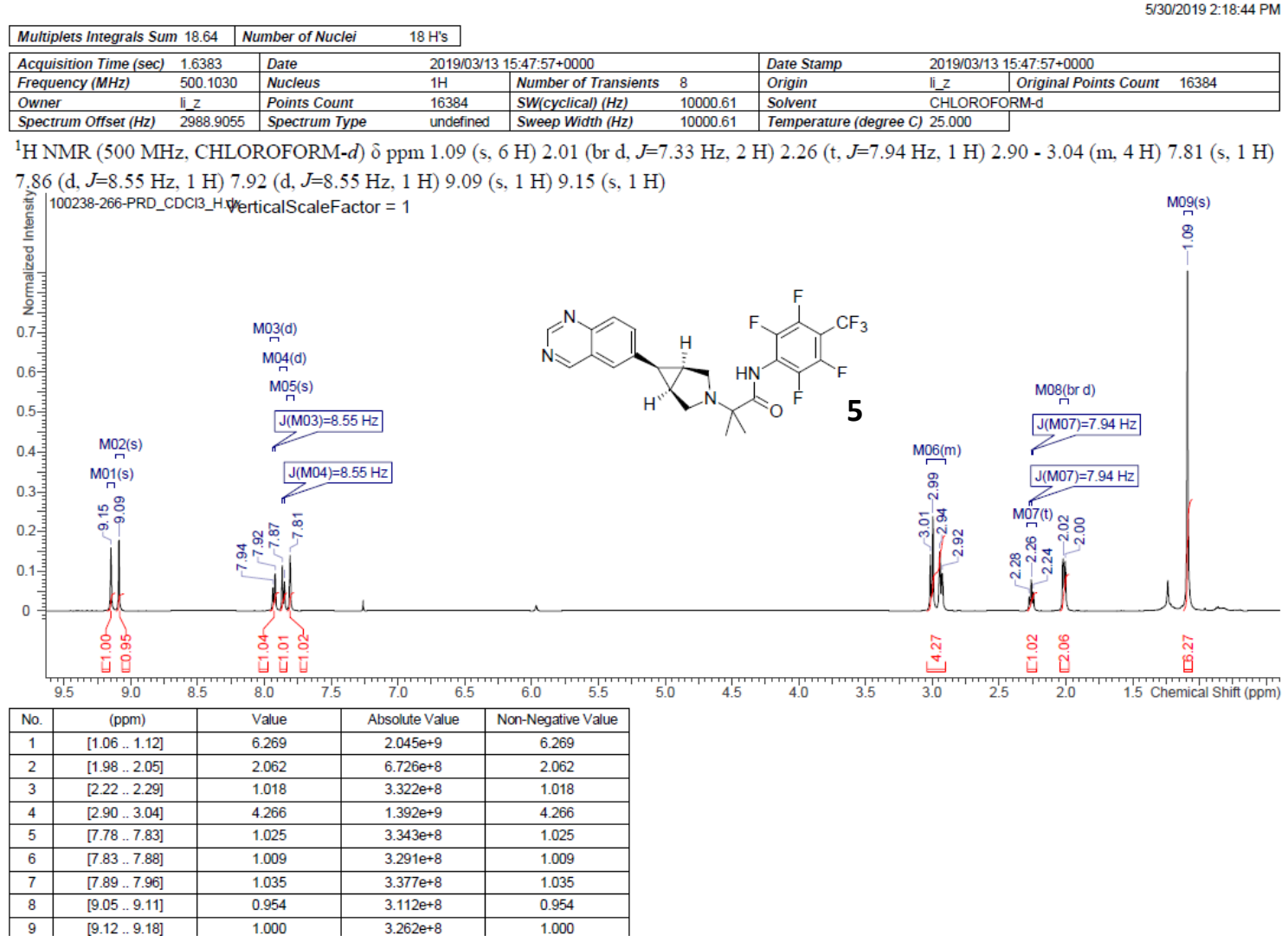

5/30/2019 2:24:37 PM

\begin{tabular}{|l|l|}
\hline Multiplets Integrals Sum 0.00 & Number of Nuclei 7 F's \\
\hline
\end{tabular}

\begin{tabular}{|c|c|c|c|c|c|c|c|c|c|}
\hline Acquisition Time (sec) & 0.6990 & \begin{tabular}{|l|} 
Date \\
\end{tabular} & \multicolumn{3}{|c|}{$2019 / 03 / 13$ 15:52:24+0000 } & Date Stamp & \multicolumn{3}{|c|}{$2019 / 03 / 13$ 15:52:24+0000 } \\
\hline Frequency $(\mathrm{MHz})$ & 470.5077 & Nucleus & $19 \mathrm{~F}$ & Number of Transients & 64 & Origin & li_z & Original Points Count & 65536 \\
\hline Owner & li_z & Points Count & 65536 & SW(cyclical) $(\mathrm{Hz})$ & 93751.43 & Solvent & \multicolumn{3}{|c|}{ CHLOROFORM-d } \\
\hline Spectrum Offset $(\mathrm{Hz})$ & -56467.046 & & & Spectrum Type & undefined & Sweep Width (Hz) & 93751.43 & \begin{tabular}{|l|l} 
Temperature (degree $C$ ) \\
)
\end{tabular} & 25.002 \\
\hline
\end{tabular}

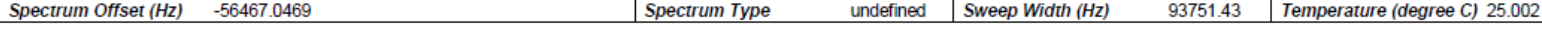

${ }^{19} \mathrm{~F}$ NMR (471 MHz, CHLOROFORM- $d$ ) $\delta \mathrm{ppm}-143.76--143.48(\mathrm{~m}, 2 \mathrm{~F})-140.95--140.60$ (m, 2 F) $-56.23(\mathrm{t}, \mathrm{J}=21.46 \mathrm{~Hz}, 3 \mathrm{~F})$

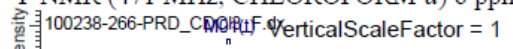

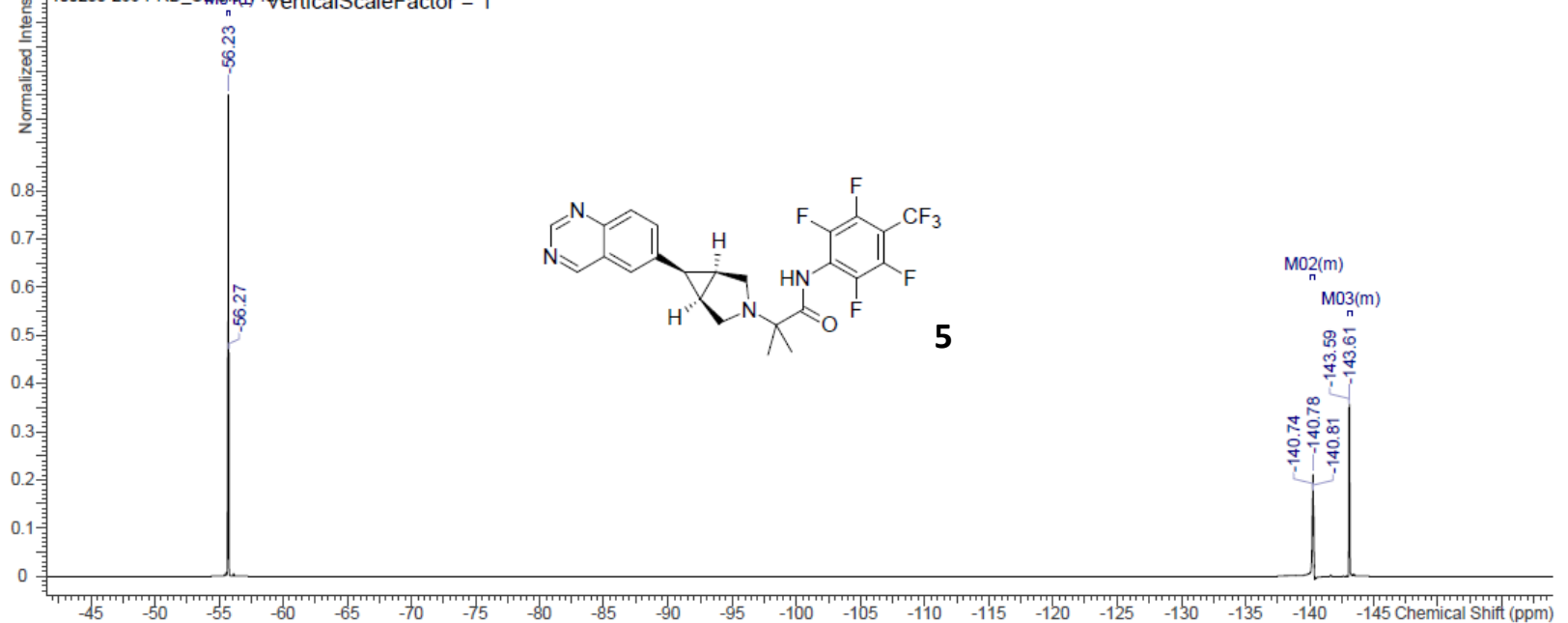

\begin{tabular}{|c|c|c|c|c|c|c|}
\hline No. & $\begin{array}{c}\text { Shift1 } \\
(\mathrm{ppm})\end{array}$ & F's & Type & $\begin{array}{c}\text { J } \\
(\mathrm{Hz})\end{array}$ & Multiplet1 & (ppm) \\
\hline 1 & -143.60 & 2 & $\mathrm{~m}$ & - & M03 & {$[-143.76 \ldots-143.48]$} \\
\hline 2 & -140.76 & 2 & $\mathrm{~m}$ & - & M02 & {$[-140.95 \ldots-140.60]$} \\
\hline 3 & -56.23 & 3 & $\mathrm{t}$ & 21.46 & M01 & {$[-56.35 \ldots-56.09]$} \\
\hline
\end{tabular}




\begin{tabular}{|ll|ll|ll|l|l|}
\hline \hline Acquisition Time $(\mathrm{sec})$ & 1.1010 & Date & $2019 / 03 / 13$ & $16: 14: 26+0000$ & Date Stamp & $2019 / 03 / 13$ 16:14:26+0000 \\
\hline Frequency $(\mathrm{MHz})$ & 125.7628 & Nucleus & $13 \mathrm{C}$ & Number of Transients & 512 & Origin & li_z \\
\hline Owner & li_z & Points Count & 32768 & SW(cyclical) $(\mathrm{Hz})$ & 29762.81 & Solvent & CHLOROFORM-d \\
\hline Spectrum Offset $(\mathrm{Hz})$ & 12589.1299 & Spectrum Type & undefined & Sweep Width $(\mathrm{Hz})$ & 29762.81 & Temperature (degree C) 25.002 \\
\hline
\end{tabular}
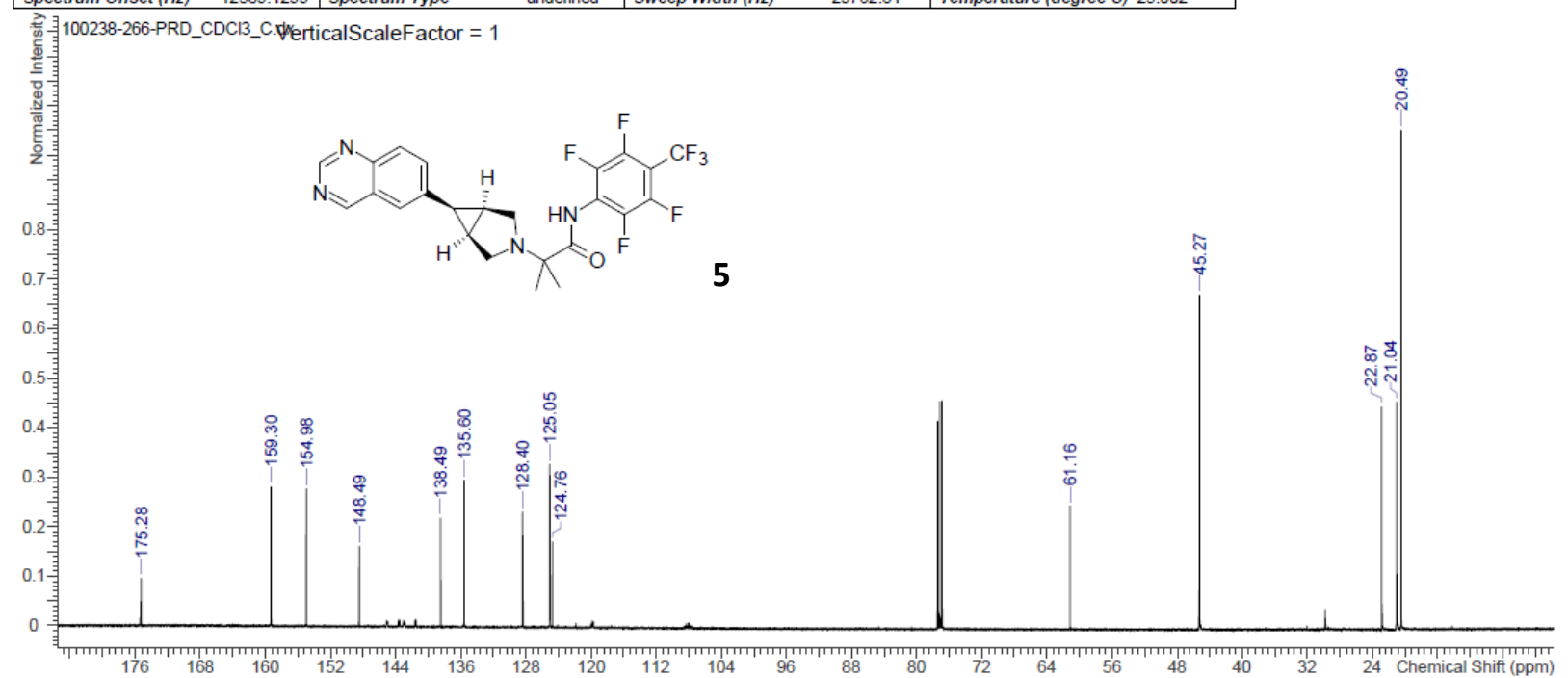

\begin{tabular}{|c|c|c|c|c|c|c|c|c|c|c|c|c|c|c|c|}
\hline No. & $(\mathrm{ppm})$ & $(\mathrm{Hz})$ & Height & No. & $(\mathrm{ppm})$ & $(\mathrm{Hz})$ & Height & No. & $(\mathrm{ppm})$ & $(\mathrm{Hz})$ & Height & No. & $(\mathrm{ppm})$ & $(\mathrm{Hz})$ & Height \\
\hline 1 & 20.49 & 2577.2 & 1.0000 & 5 & 61.16 & 7691.0 & 0.2422 & 9 & 135.60 & 17053.1 & 0.2932 & 13 & 159.30 & 20034.1 & 0.2799 \\
\hline 2 & 21.04 & 2646.2 & 0.4515 & 6 & 124.76 & 15690.6 & 0.1699 & 10 & 138.49 & 17416.4 & 0.2174 & 14 & 175.28 & 22044.3 & 0.0955 \\
\hline 3 & 22.87 & 2876.0 & 0.4423 & 7 & 125.05 & 15726.9 & 0.3260 & 11 & 148.49 & 18674.4 & 0.1605 & & & & \\
\hline 4 & 45.27 & 5692.7 & 0.6682 & 8 & 128.40 & 16147.5 & 0.2291 & 12 & 154.98 & 19491.0 & 0.2757 & & & & \\
\hline
\end{tabular}

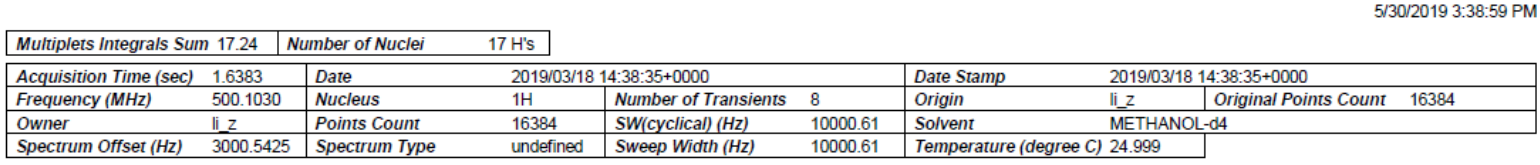

${ }^{1} \mathrm{H}$ NMR $(500 \mathrm{MHz}$, METHANOL-d 4 ) $\delta \mathrm{ppm} 1.08(\mathrm{~s}, 6 \mathrm{H}) 2.05-2.10(\mathrm{~m}, 2 \mathrm{H}) 2.23(\mathrm{t}, J=7.94 \mathrm{~Hz}, 1 \mathrm{H}) 2.95-3.00(\mathrm{~m}, 2 \mathrm{H}) 3.10(\mathrm{~d}, J=9.16 \mathrm{~Hz}, 2$ H) $4.88(\mathrm{~s}, 1 \mathrm{H}) 8.38(\mathrm{~d}, J=3.66 \mathrm{~Hz}, 1 \mathrm{H}) 8.46(\mathrm{dd}, J=2.75,1.53 \mathrm{~Hz}, 1 \mathrm{H}) 8.67(\mathrm{~s}, 1 \mathrm{H})$

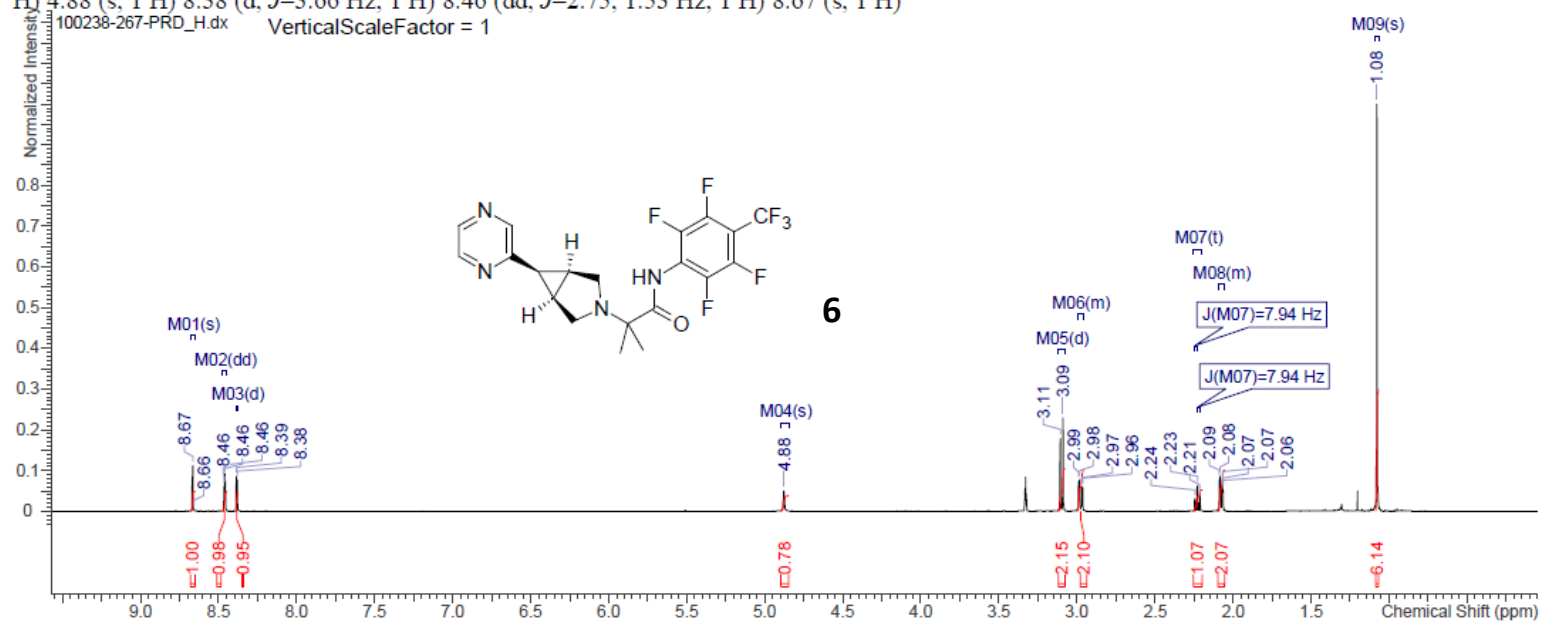

\begin{tabular}{|c|c|c|c|c|}
\hline No. & $(\mathrm{ppm})$ & Value & Absolute Value & Non-Negative Value \\
\hline 1 & {$[1.07 . .1 .09]$} & 6.136 & $1.129 \mathrm{e}+9$ & 6.136 \\
\hline 2 & {$[2.05 \ldots 2.10]$} & 2.070 & $3.808 \mathrm{e}+8$ & 2.070 \\
\hline 3 & {$[2.20 \ldots 2.25]$} & 1.072 & $1.971 \mathrm{e}+8$ & 1.072 \\
\hline 4 & {$[2.95 \ldots 3.00]$} & 2.099 & $3.861 \mathrm{e}+8$ & 2.099 \\
\hline 5 & {$[3.08 \ldots 3.12]$} & 2.152 & $3.958 \mathrm{e}+8$ & 2.152 \\
\hline 6 & {$[4.85 \ldots 4.90]$} & 0.779 & $1.433 \mathrm{e}+8$ & 0.779 \\
\hline 7 & {$[8.37 . .8 .39]$} & 0.955 & $1.756 \mathrm{e}+8$ & 0.955 \\
\hline 8 & {$[8.45 .8 .47]$} & 0.981 & $1.805 \mathrm{e}+8$ & 0.981 \\
\hline 9 & {$[8.65 \ldots 8.68]$} & 1.000 & $1.839 \mathrm{e}+8$ & 1.000
\end{tabular}




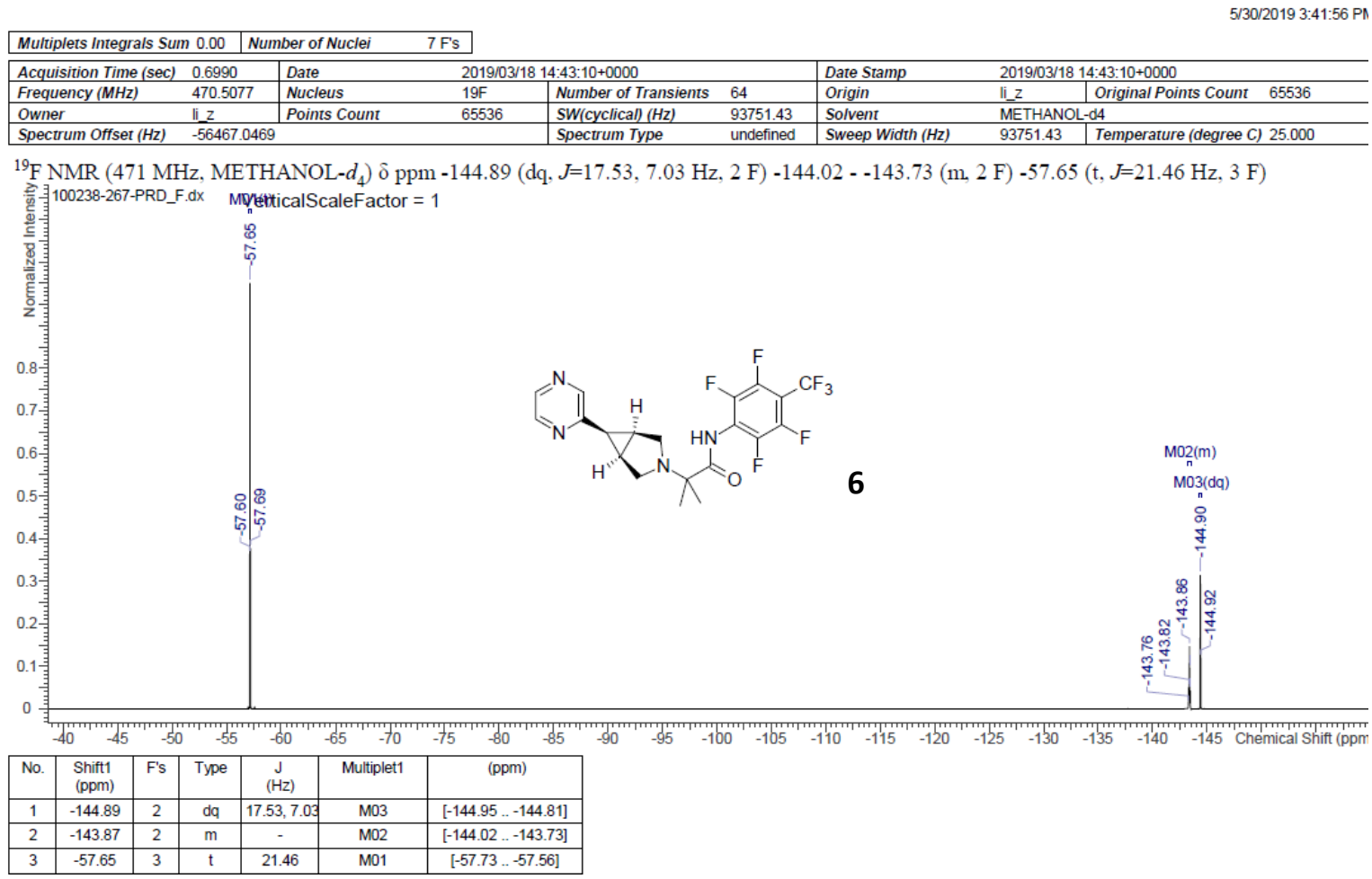

5/30/2019 3:44:33 PM

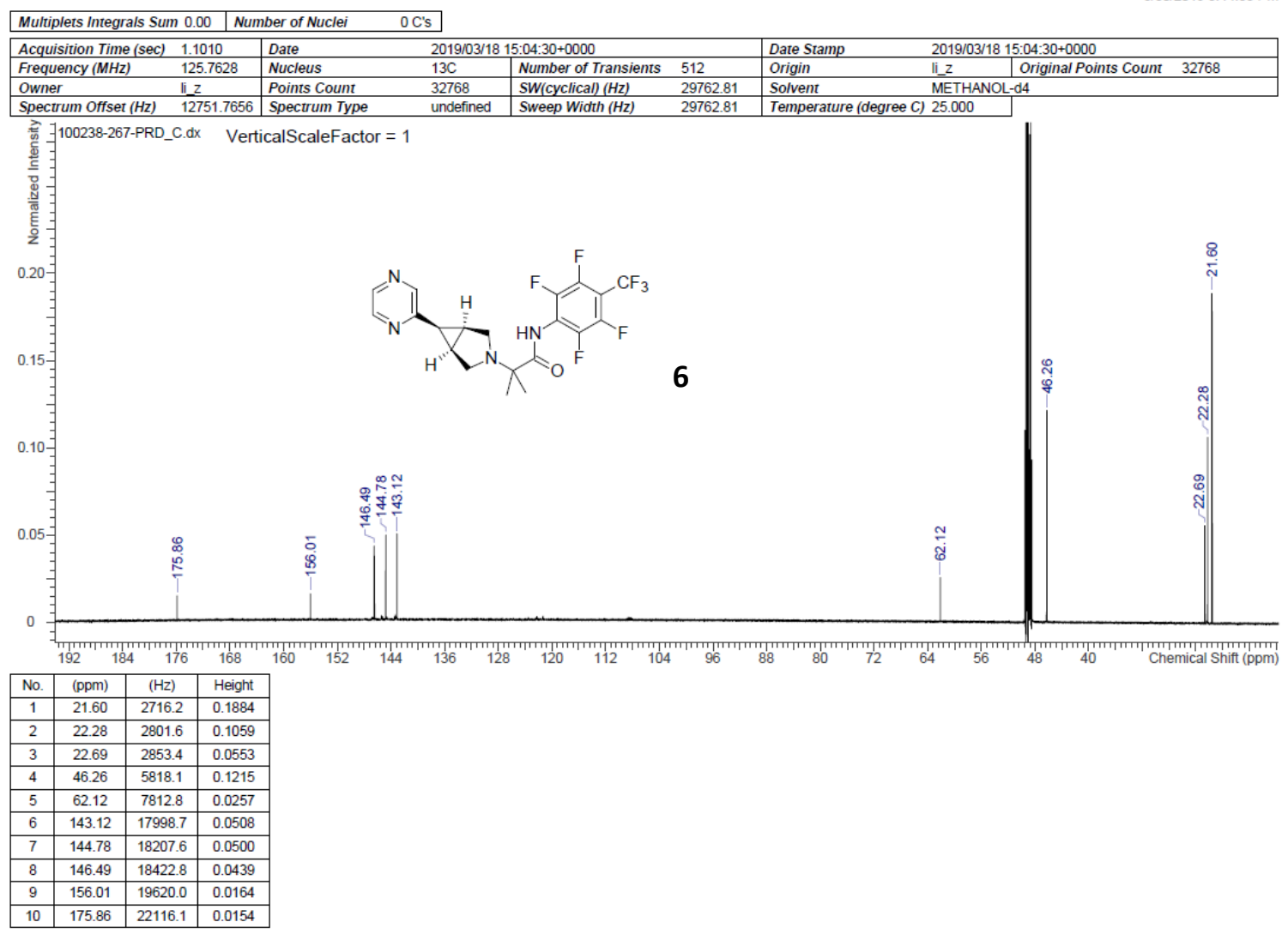




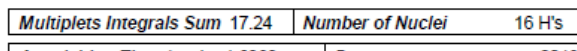

\begin{tabular}{|c|c|c|c|c|c|c|c|c|c|}
\hline Acquisition Time (sec) & 1.6383 & Date & \multicolumn{3}{|c|}{$2019 / 05 / 2220: 27: 11+0000$} & \multicolumn{4}{|c|}{$2019 / 05 / 2220: 27: 11+0000$} \\
\hline Frequency $(\mathrm{MHz})$ & 500.1030 & Nucleus & $1 \mathrm{H}$ & Number of Transients & 8 & Origin & li_z & Original Points Count & 16384 \\
\hline Owner & li_z & Points Count & 16384 & SW(cyclical) $(\mathrm{Hz})$ & 10000.61 & Solvent & CHLOR & RM-d & \\
\hline Spectrum Offset $(\mathrm{Hz})$ & 2988.8904 & Spectrum Type & undefined & Sweep Width $(\mathrm{Hz})$ & 10000.61 & Temperature (degree $\mathrm{C}$ ) & 24.999 & & \\
\hline
\end{tabular}

${ }^{1} \mathrm{H}$ NMR (500 MHz, CHLOROFORM-d) $\delta \mathrm{ppm} 1.19(\mathrm{~s}, 6 \mathrm{H}) 2.00-2.08(\mathrm{~m}, 3 \mathrm{H}) 2.90-2.98(\mathrm{~m}, 2 \mathrm{H}) 3.02(\mathrm{~d}, \mathrm{~J}=9.77 \mathrm{~Hz}, 2 \mathrm{H}) 6.74(\mathrm{~s}, 1 \mathrm{H}) 6.93$ (d. $J=3.66 \mathrm{~Hz}, 1 \mathrm{H}) 7.31(\mathrm{~d}, J=3.66 \mathrm{~Hz}, 1 \mathrm{H})$

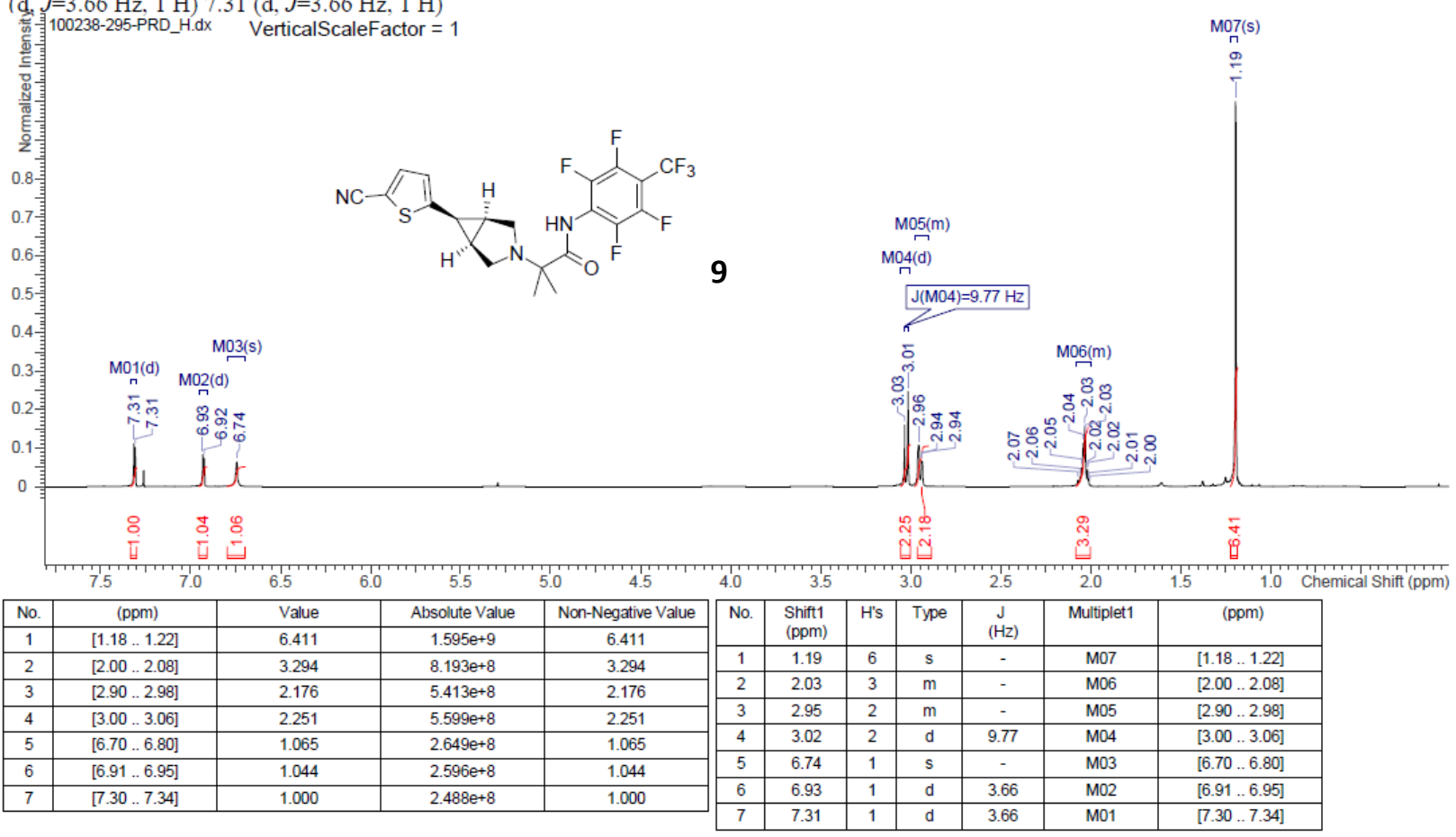

\begin{tabular}{|l|l|}
\hline Multiplets Integrals Sum 0.00 & Number of Nuclei \\
\hline
\end{tabular}

5/31/2019 10:28:51 AM

\begin{tabular}{|ll|ll|ll|l|l|l|}
\hline Acquisition Time $(\mathrm{sec})$ & 0.6990 & Date & $2019 / 05 / 22$ & $20: 31: 08+0000$ & Date Stamp & $2019 / 05 / 2220: 31: 08+0000$ \\
\hline Frequency $(\mathrm{MHz})$ & 470.5077 & Nucleus & $19 \mathrm{~F}$ & Number of Transients & 64 & Origin & li_z & Original Points Count $\quad 65536$ \\
\hline Owner & li z & Points Count & 65536 & SW(cyclical) $(\mathrm{Hz})$ & 93751.43 & \multicolumn{2}{|l|}{ Solvent } & CHLOROFORM-d \\
\hline Spectrum Offset $(\mathrm{Hz})$ & -56467.0469 & & Spectrum Type & undefined & Sweep Width (Hz) & 93751.43 & Temperature (degree C) 25.002 \\
\hline
\end{tabular}

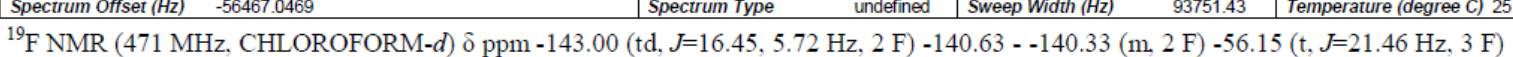

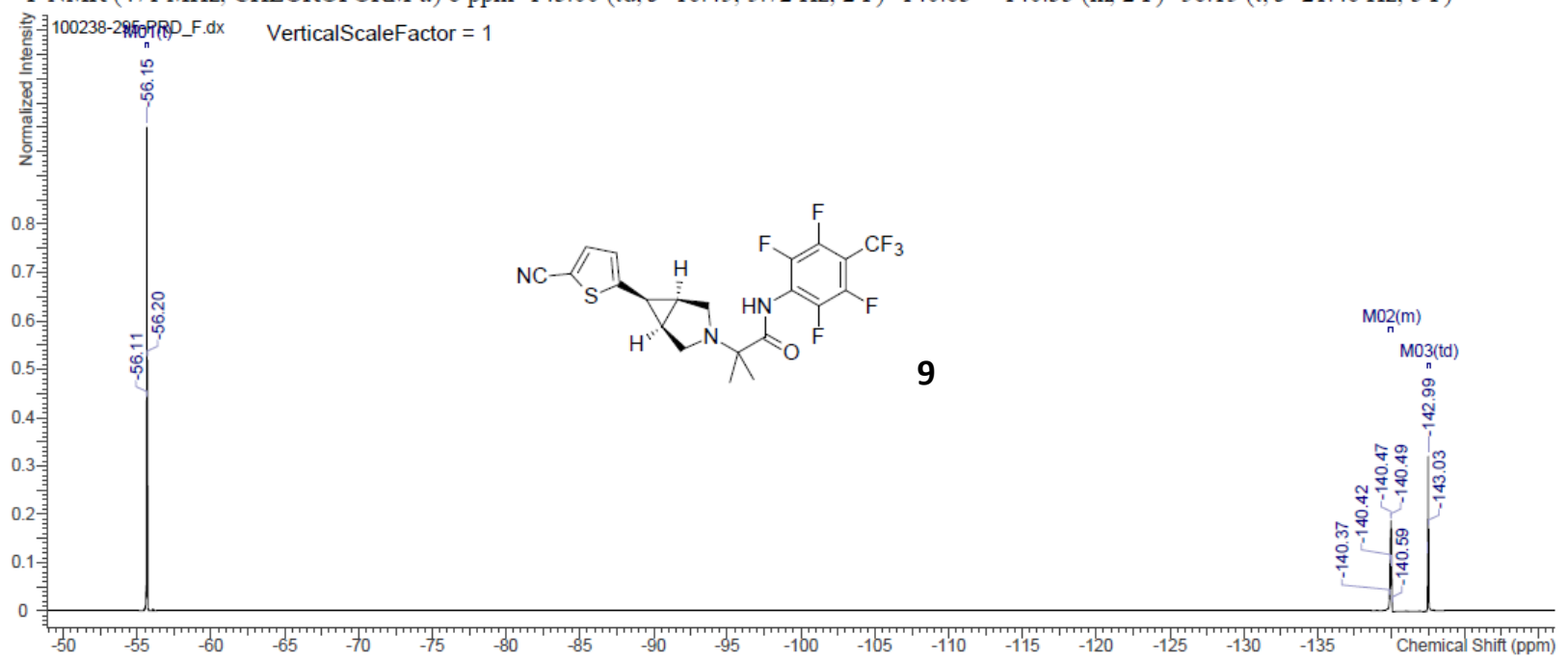

\begin{tabular}{|c|c|c|c|c|c|c|}
\hline No. & $\begin{array}{c}\text { Shift1 } \\
(\mathrm{ppm})\end{array}$ & F's & Type & $\begin{array}{c}\mathrm{J} \\
(\mathrm{Hz})\end{array}$ & Multiplet1 & (ppm) \\
\hline 1 & -143.00 & 2 & td & $16.45,5.72$ & M03 & {$[-143.11 \ldots-142.90]$} \\
\hline 2 & -140.48 & 2 & $\mathrm{~m}$ & - & M02 & {$[-140.63 \ldots-140.33]$} \\
\hline 3 & -56.15 & 3 & $\mathrm{t}$ & 21.46 & M01 & {$[-56.25 \ldots-56.06]$} \\
\hline
\end{tabular}



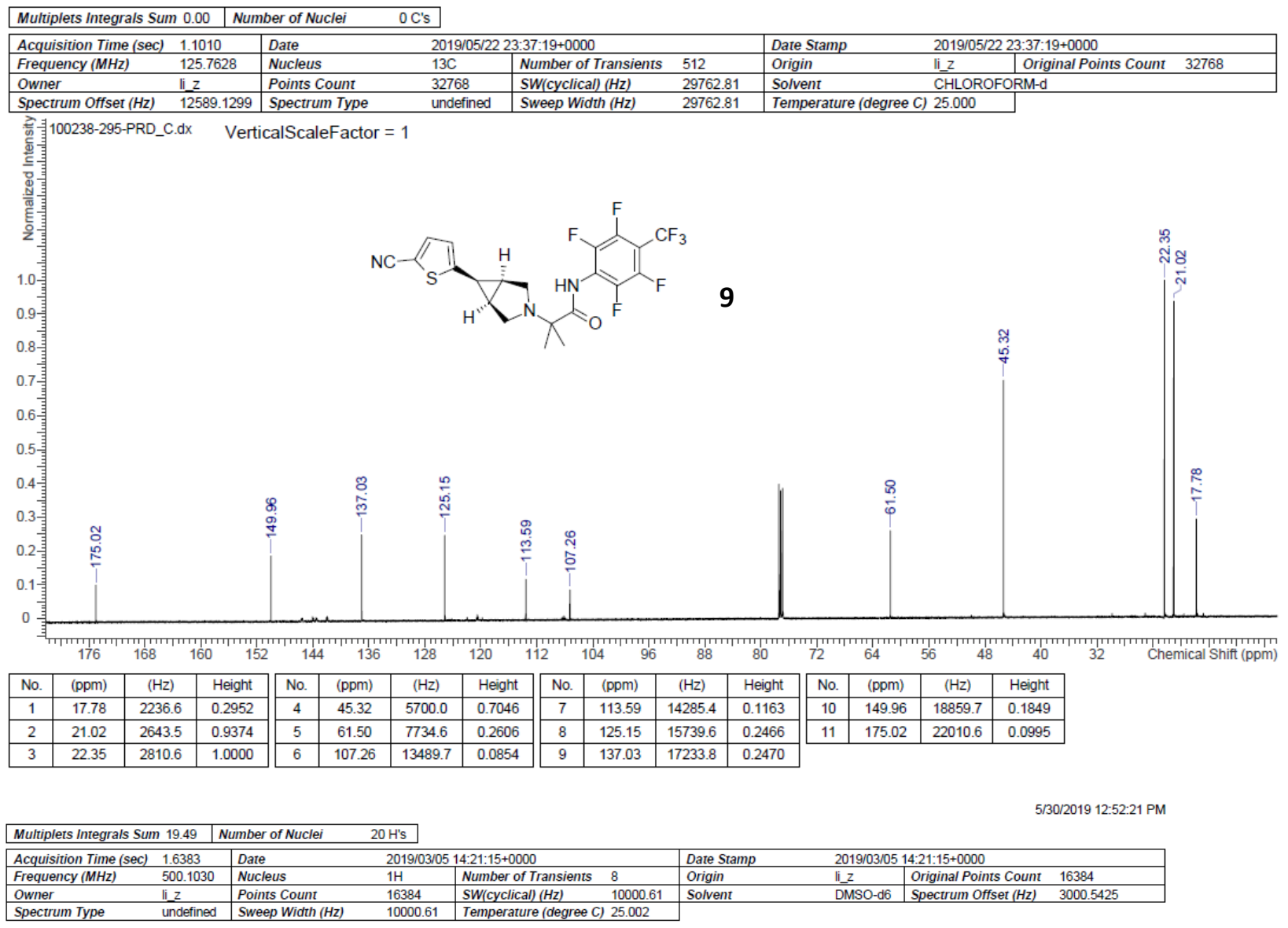

${ }^{1} \mathrm{H}$ NMR $\left(500 \mathrm{MHz}, \mathrm{DMSO}-d_{6}\right) \delta \mathrm{ppm} 1.02(\mathrm{~s}, 6 \mathrm{H}) 1.83-1.89(\mathrm{~m}, 2 \mathrm{H}) 2.12(\mathrm{t}, J=7.94 \mathrm{~Hz}, 1 \mathrm{H}) 2.85$ (br d, J=8.55 Hz, $\left.2 \mathrm{H}\right) 2.95$ (d, J=8.55 Hz, 2 管) $5.95-6.00(\mathrm{~m}, 1 \mathrm{H}) 6.13(\mathrm{~s}, 1 \mathrm{H}) 6.84-6.88(\mathrm{~m}, 1 \mathrm{H}) 7.11(\mathrm{~d}, J=7.94 \mathrm{~Hz}, 1 \mathrm{H}) 7.25(\mathrm{~d}, J=7.94 \mathrm{~Hz}, 1 \mathrm{H}) 7.36(\mathrm{~s}, 1 \mathrm{H}) 10.87$ (br s, $1 \mathrm{H})$

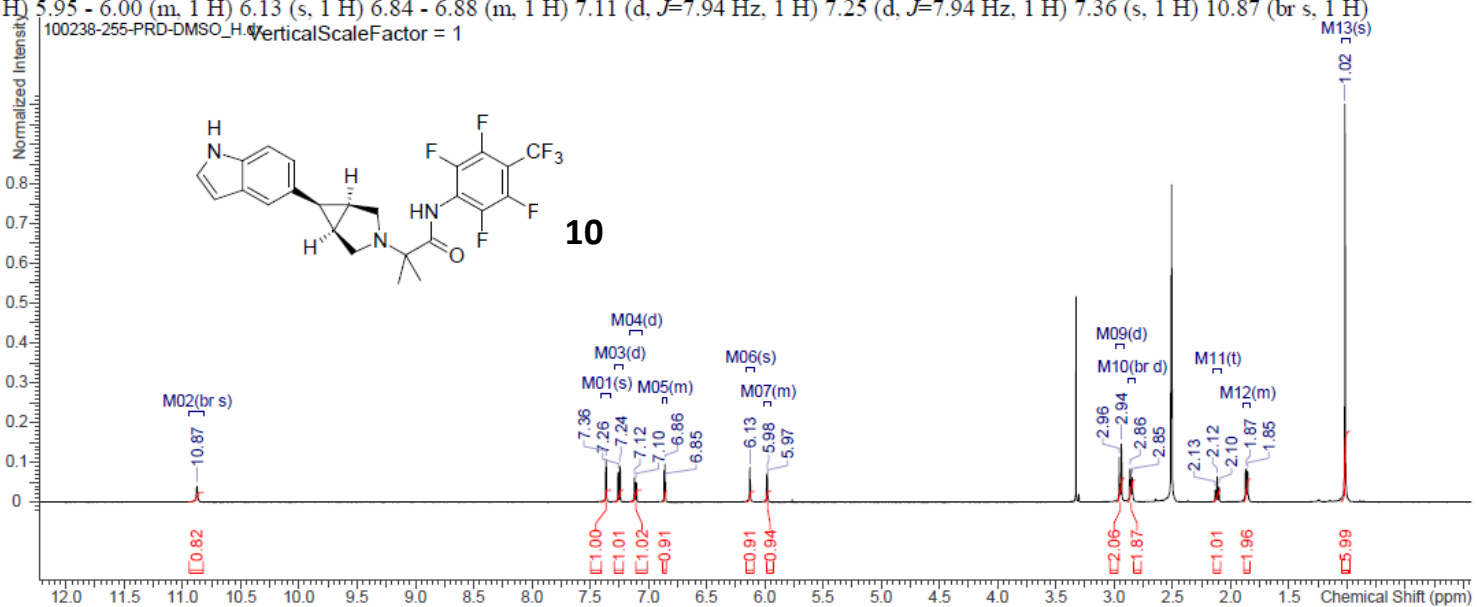

\begin{tabular}{|c|c|c|c|c|c|c|c|c|c|c|c|c|c|c|c|c|}
\hline No. & $\begin{array}{l}\text { Shift1 } \\
(\mathrm{ppm})\end{array}$ & H's & Type & $\begin{array}{c}\mathrm{J} \\
(\mathrm{Hz})\end{array}$ & Multiplet1 & \multicolumn{2}{|c|}{ (ppm) } & No. & $\begin{array}{l}\text { Shift } \\
\text { (ppn }\end{array}$ & & H's & Type & $\begin{array}{c}\mathrm{J} \\
(\mathrm{Hz})\end{array}$ & Multiplet1 & (ppm) & \\
\hline 1 & 1.02 & 6 & $\mathrm{~s}$ & - & M13 & \multicolumn{2}{|c|}{$[0.98 \ldots 1.05]$} & 7 & 6.1 & & 1 & $\mathrm{~s}$ & - & M06 & {$[6.09 \ldots 6.16]$} & \\
\hline 2 & 1.86 & 2 & $\mathrm{~m}$ & - & M12 & \multicolumn{2}{|c|}{$[1.83 \ldots 1.89]$} & 8 & 6.8 & & 1 & $\mathrm{~m}$ & - & M05 & {$[6.84 \ldots 6.88]$} & \\
\hline 3 & 2.12 & 1 & $\mathrm{t}$ & 7.94 & M11 & \multicolumn{2}{|c|}{$[2.08 \ldots 2.15]$} & 9 & 7.1 & & 1 & d & 7.94 & M04 & {$[7.06 \ldots 7.16]$} & \\
\hline 4 & 2.85 & 2 & brd & 8.55 & M10 & \multicolumn{2}{|c|}{$[2.82 \ldots 2.88]$} & 10 & 7.2 & & 1 & d & 7.94 & M03 & {$[7.22 \ldots .7 .29]$} & \\
\hline 5 & 2.95 & 2 & d & 8.55 & M09 & \multicolumn{2}{|c|}{$[2.91 \ldots 2.98]$} & 11 & 7.3 & & 1 & $\mathrm{~s}$ & - & M01 & [7.32 . . 7.41] & \\
\hline 6 & 5.98 & 1 & $\mathrm{~m}$ & - & M07 & \multicolumn{2}{|c|}{$[5.95 \ldots 6.00]$} & 12 & 10.8 & & 1 & brs & - & M02 & {$[10.82 \ldots 10.94]$} & \\
\hline No. & \multicolumn{3}{|c|}{ (ppm) } & Value & \multicolumn{2}{|c|}{ Absolute Value } & & ative 1 & & No. & & \multicolumn{2}{|c|}{ (ppm) } & Value & Absolute Value & Non-Negative Value \\
\hline 1 & \multicolumn{2}{|c|}{$[0.98 \ldots 1.05]$} & & 5.994 & \multicolumn{2}{|c|}{$2.107 \mathrm{e}+8$} & & 994 & & 2 & & {$[1.83 \ldots$} & & 1.964 & $6.905 \mathrm{e}+7$ & 1.964 \\
\hline
\end{tabular}


\begin{tabular}{|l|ll|}
\hline Multiplets Integrals Sum 0.00 & Number of Nuclei & $7 \mathrm{~F}$ 's \\
\hline
\end{tabular}
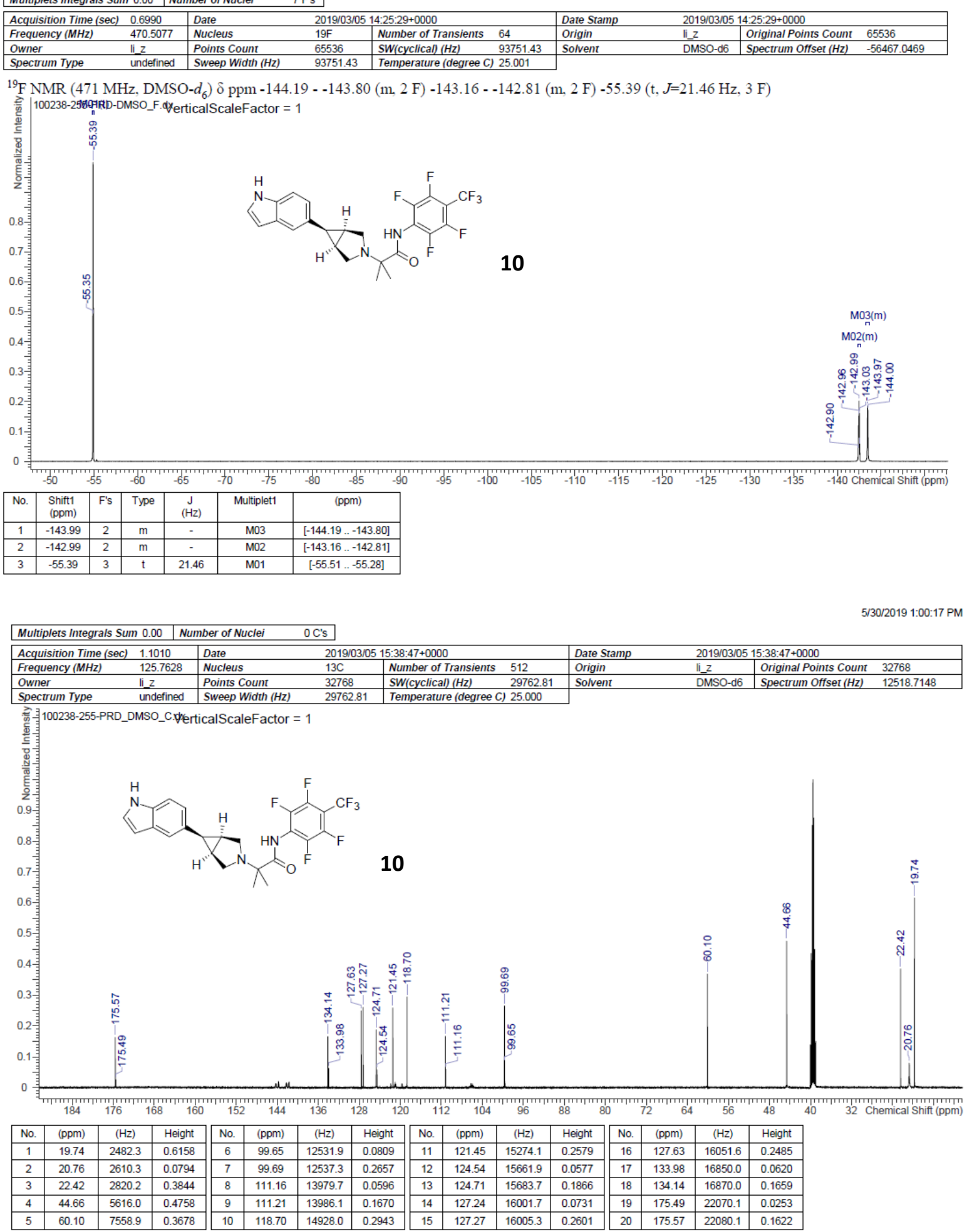


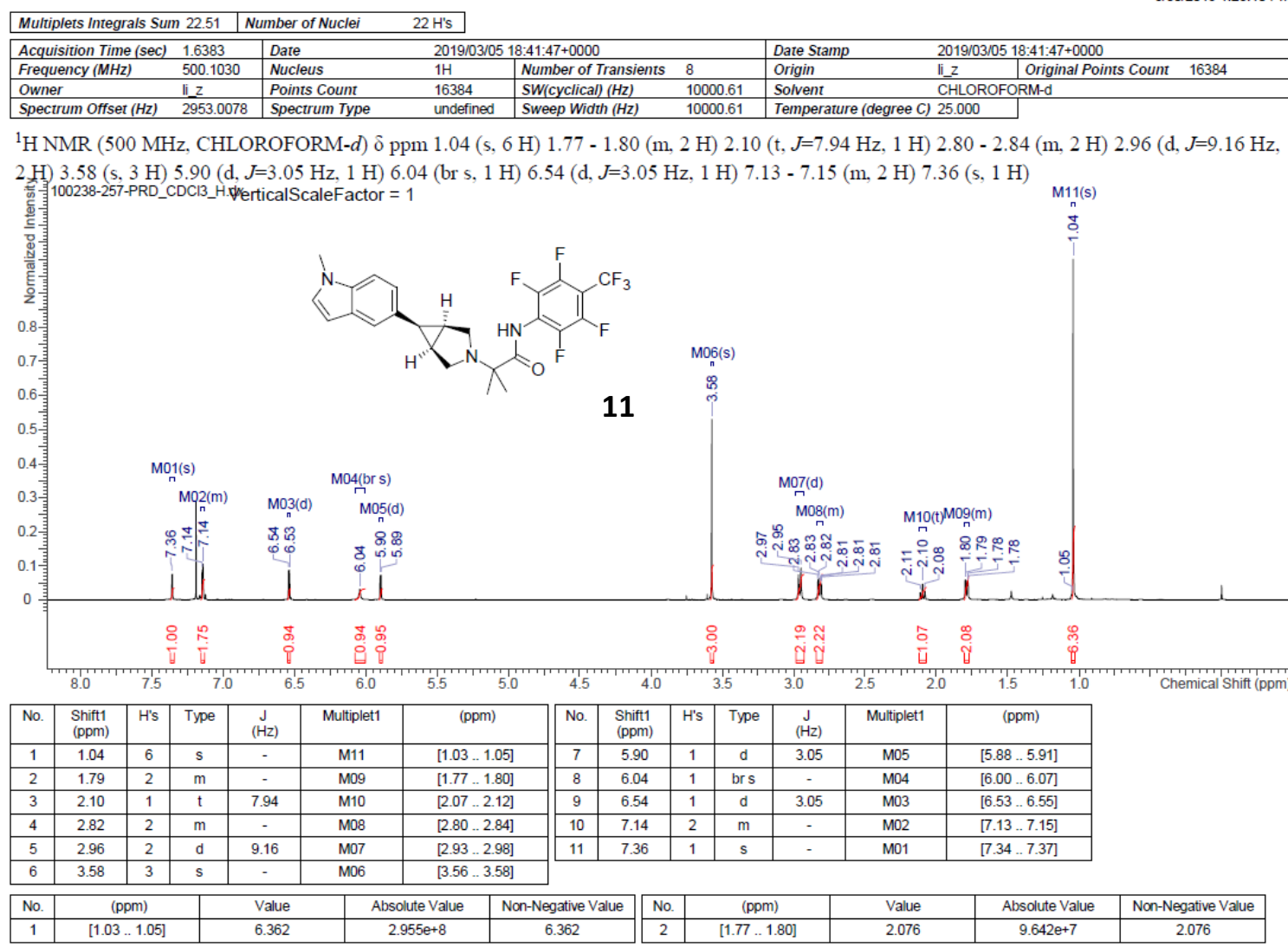

5/30/2019 1:30:04 PM

\begin{tabular}{|l|ll|}
\hline Multiplets Integrals Sum 0.00 & Number of Nuclei & $7 \mathrm{~F}$ 's \\
\hline
\end{tabular}

\begin{tabular}{|ll|ll|ll|l|l|l|}
\hline Acquisition Time $(\mathrm{sec})$ & 0.6990 & Date & $2019 / 03 / 05$ & $18: 45: 33+0000$ & Date Stamp & $2019 / 03 / 05$ 18:45:33+0000 \\
\hline Frequency $(\mathrm{MHz})$ & 470.5077 & Nucleus & 19F & Number of Transients & 64 & Origin & li_z \\
\hline Owner & li_z & Points Count & 65536 & SW(cyclical) $(\mathrm{Hz})$ & 93751.43 & Solvent & CHLOROFORM-d \\
\hline Spectrum Offset $(\mathrm{Hz})$ & -56467.0469 & & Spectrum Type & undefined & Sweep Width (Hz) & 93751.43 & Temperature (degree C) 25.000 \\
\hline
\end{tabular}

${ }^{19} \mathrm{~F}$ NMR (471 MHz, CHLOROFORM- $d$ ) $\delta \mathrm{ppm}-144.08$ (br s, $\left.2 \mathrm{~F}\right)-143.09-\mathbf{- 1 4 2 . 7 9}$ (m, $\left.2 \mathrm{~F}\right)-56.22(\mathrm{t}, J=22.17 \mathrm{~Hz}, 3 \mathrm{~F})$

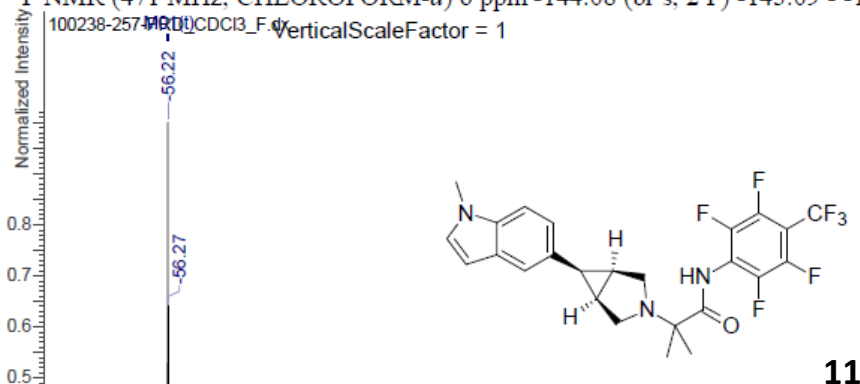

11

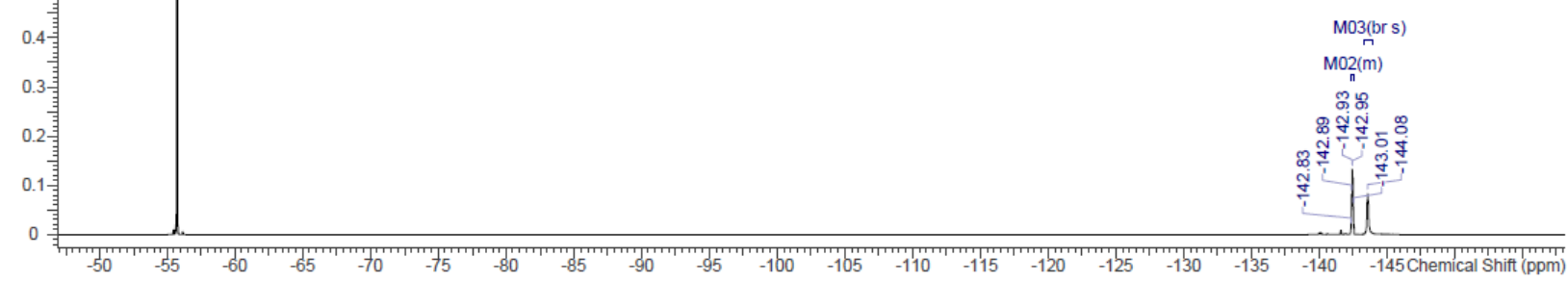

\begin{tabular}{|c|c|c|c|c|c|c|}
\hline No. & $\begin{array}{c}\text { Shift1 } \\
(\mathrm{ppm})\end{array}$ & F's & Type & $\begin{array}{c}\text { J } \\
(\mathrm{Hz})\end{array}$ & Multiplet1 & $(\mathrm{ppm})$ \\
\hline 1 & -144.08 & 2 & $\mathrm{br} \mathrm{s}$ & - & M03 & {$[-144.46 \ldots-143.79]$} \\
\hline 2 & -142.94 & 2 & $\mathrm{~m}$ & - & M02 & {$[-143.09 \ldots-142.79]$} \\
\hline 3 & -56.22 & 3 & $\mathrm{t}$ & 22.17 & M01 & {$[-56.29 \ldots-56.15]$} \\
\hline
\end{tabular}




\begin{tabular}{|c|c|c|c|c|c|c|c|c|c|}
\hline Multiplets Integrals Sun & 0.00 & nber of Nuclei & $0 \mathrm{C}$ 's & & & & & & \\
\hline Acquisition Time (sec) & 1.1010 & Date & $2019 / 03 / 05$ & $9: 21: 25+0000$ & & Date Stamp & $2019 / 03$ & $9: 21: 25+0000$ & \\
\hline Frequency $(\mathrm{MHz})$ & 125.7628 & Nucleus & $13 \mathrm{C}$ & Number of Transients & 512 & Origin & lizz & Original Points Count & 32768 \\
\hline Owner & li_z & Points Count & 32768 & SW(cyclical) (Hz) & 29762.81 & Solvent & $\mathrm{CHLOR}$ & RM-d & \\
\hline Spectrum Offset $(\mathrm{Hz})$ & 12590.0352 & Spectrum Type & undefined & Sweep Width $(\mathrm{Hz})$ & 29762.81 & Temperature (degree $C$ & 24.999 & & \\
\hline
\end{tabular}

煫寻100238-257-PRD_C.dx VerticalScaleFactor $=1$

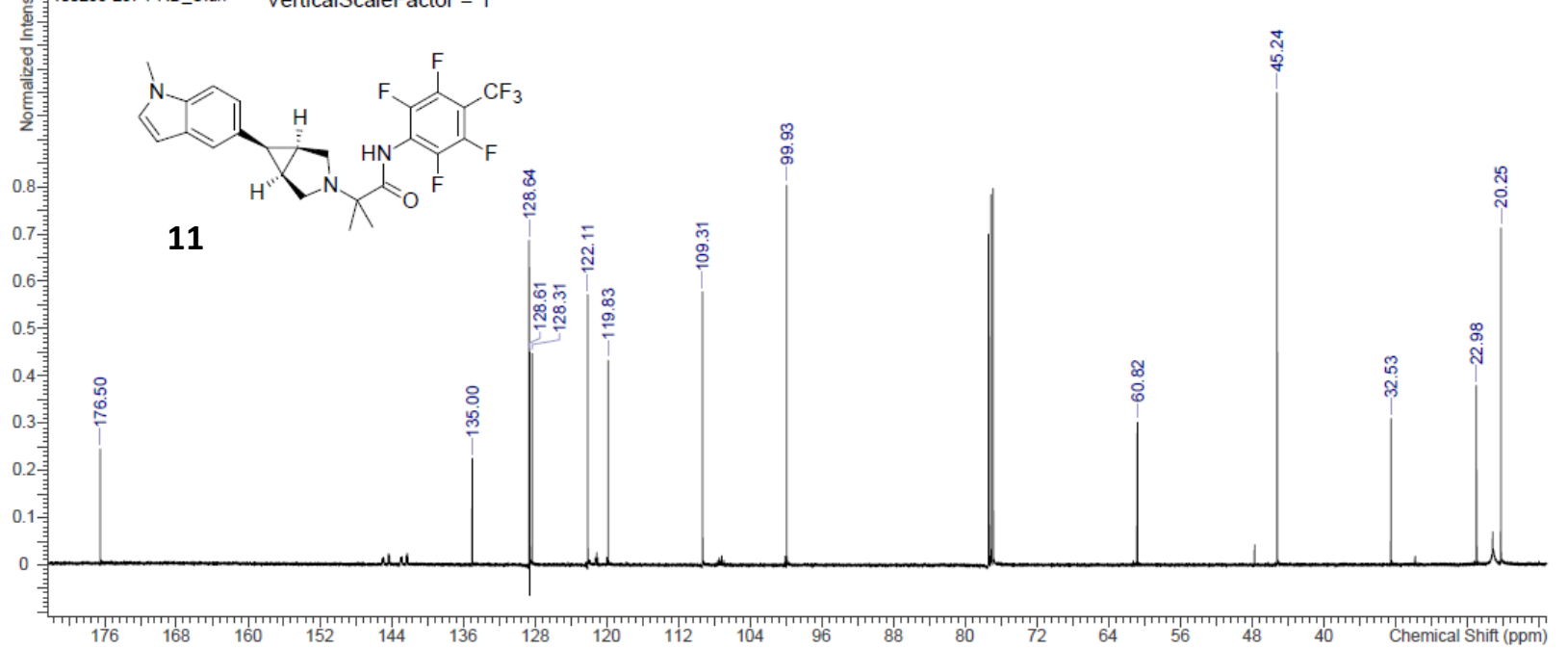

\begin{tabular}{|c|c|c|c|c|c|c|c|c|c|c|c|c|c|c|c|}
\hline No. & (ppm) & $(\mathrm{Hz})$ & Height & No. & (ppm) & $(\mathrm{Hz})$ & Height & No. & $(\mathrm{ppm})$ & $(\mathrm{Hz})$ & Height & No. & $(\mathrm{ppm})$ & $(\mathrm{Hz})$ & Height \\
\hline 1 & 20.25 & 2547.2 & 0.7131 & 5 & 60.82 & 7649.2 & 0.3016 & 9 & 122.11 & 15357.2 & 0.5711 & 13 & 135.00 & 16977.7 & 0.2250 \\
\hline 2 & 22.98 & 2890.6 & 0.3792 & 6 & 99.93 & 12566.9 & 0.8029 & 10 & 128.31 & 16136.6 & 0.4465 & 14 & 176.50 & 22197.8 & 0.2445 \\
\hline 3 & 32.53 & 4090.5 & 0.3096 & 7 & 109.31 & 13747.7 & 0.5773 & 11 & 128.61 & 16173.8 & 0.4499 & & & & \\
\hline 4 & 45.24 & 5689.1 & 1.0000 & 8 & 119.83 & 15070.2 & 0.4319 & 12 & 128.64 & 16178.3 & 0.6869 & & & & \\
\hline
\end{tabular}

5/30/2019 10:13:44 AM

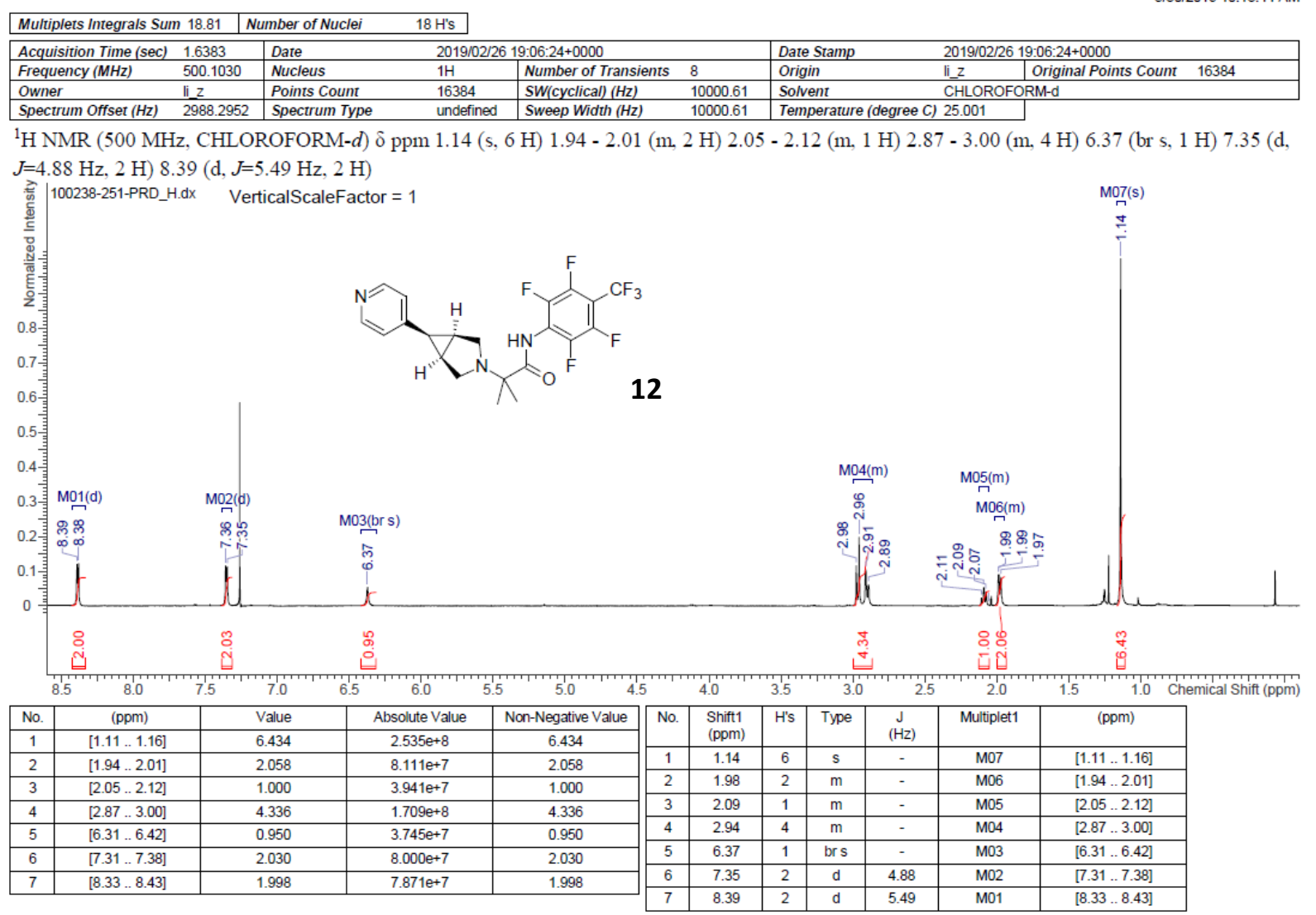


\begin{tabular}{|l|l|}
\hline Multiplets Integrals Sum 0.00 & Number of Nuclei 7 F's \\
\hline
\end{tabular}

\begin{tabular}{|c|c|c|c|c|c|c|c|c|}
\hline Acquisition Time (sec) & 0.6990 & Date & \multicolumn{3}{|c|}{ 2019/02/26 19:10:45+0000 } & Date Stamp & \multicolumn{2}{|c|}{$2019 / 02 / 26$ 19:10:45+0000 } \\
\hline Frequency $(\mathrm{MHz})$ & 470.5077 & Nucleus & $19 \mathrm{~F}$ & Number of Transients & 64 & Origin & li_z & Original Points Count 65536 \\
\hline Owner & li_z & Points Count & 65536 & SW(cyclical) $(\mathrm{Hz})$ & 93751.43 & Solvent & \multicolumn{2}{|c|}{ CHLOROFORM-d } \\
\hline Spectrum Offset (Hz) & -56467.046 & & & Spectrum Type & undefined & Sweep Width (Hz) & 93751.43 & Temperature (degree C) 25.000 \\
\hline
\end{tabular}

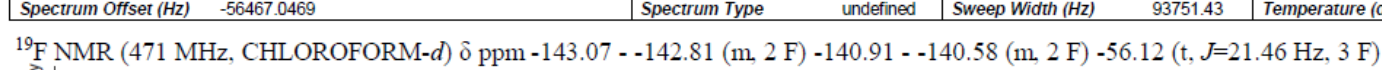

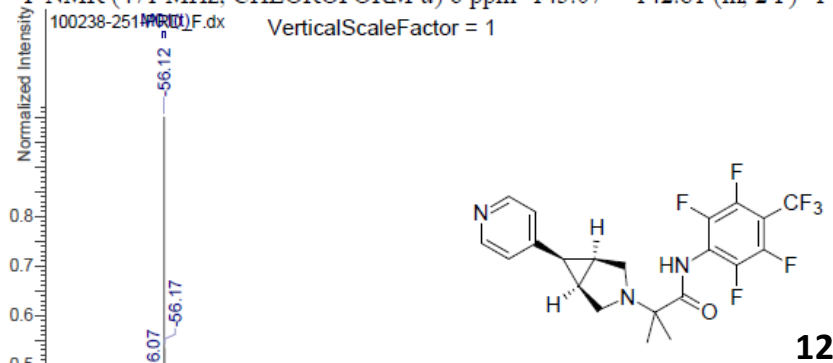

12

$\underset{\Pi}{\mathrm{M} 03(\mathrm{~m})}$ $\operatorname{M04}(\mathrm{m})$

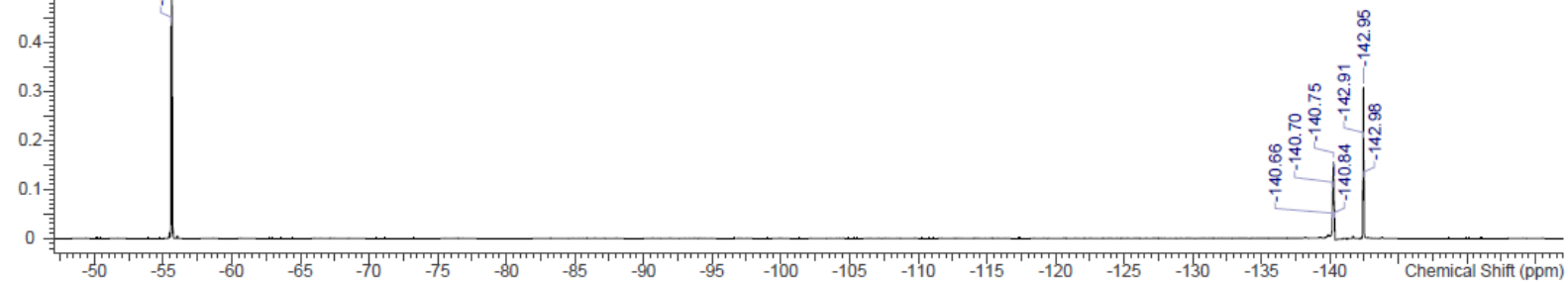

\begin{tabular}{|c|c|c|c|c|c|c|}
\hline No. & $\begin{array}{c}\text { Shift1 } \\
(\mathrm{ppm})\end{array}$ & F's & Type & $\begin{array}{c}\mathrm{J} \\
(\mathrm{Hz})\end{array}$ & Multiplet1 & $(\mathrm{ppm})$ \\
\hline 1 & -142.93 & 2 & $\mathrm{~m}$ & - & $\mathrm{M} 04$ & {$[-143.07 \ldots-142.81]$} \\
\hline 2 & -140.75 & 2 & $\mathrm{~m}$ & - & $\mathrm{M} 03$ & {$[-140.91 \ldots-140.58]$} \\
\hline 3 & -56.12 & 3 & $\mathrm{t}$ & 21.46 & M01 & {$[-56.22 \ldots-56.02]$} \\
\hline
\end{tabular}

5/30/2019 11:08:43 AM

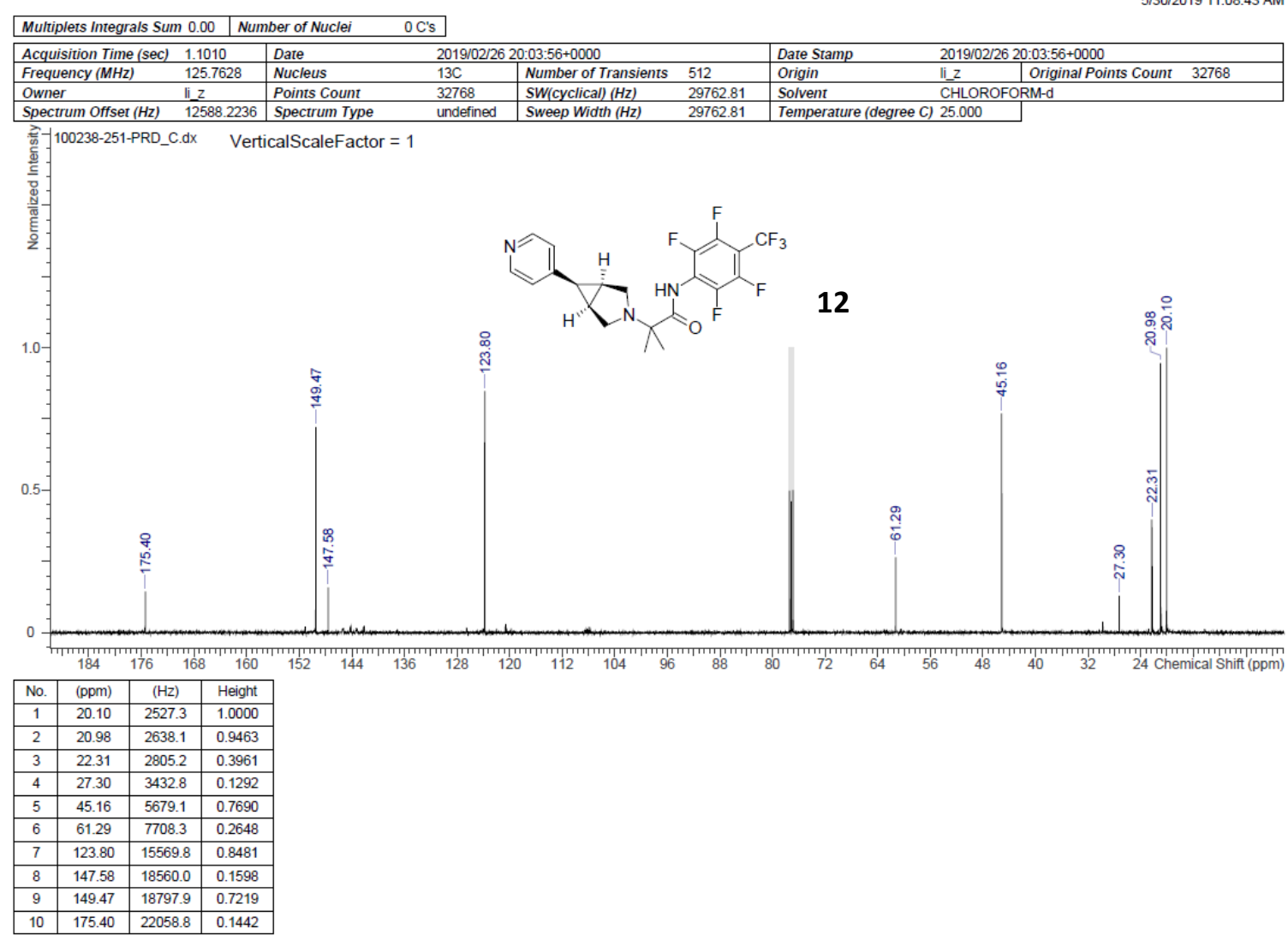


\begin{tabular}{|l|l|l|}
\hline Multiplets Integrals Sum 18.83 & Number of Nuclei $18 \mathrm{H}$ 's \\
\hline \hline
\end{tabular}

5/30/2019 11:19:36 AM

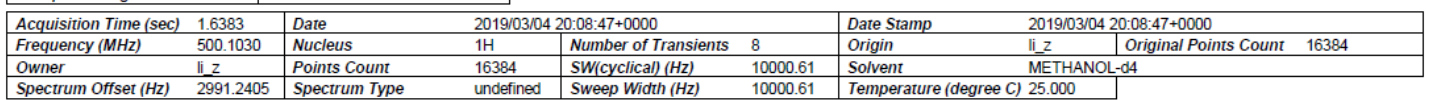

${ }^{1} \mathrm{H}$ NMR $\left(500 \mathrm{MHz}\right.$, METHANOL-d $\left.{ }_{4}\right) \delta \mathrm{ppm} 1.10(\mathrm{~s}, 6 \mathrm{H}) 1.98$ - $2.01(\mathrm{~m}, 2 \mathrm{H}) 2.07$ - $2.13(\mathrm{~m}, 1 \mathrm{H}) 2.92-3.01(\mathrm{~m}, 4 \mathrm{H}) 4.86(\mathrm{~s}, 1 \mathrm{H}) 7.32(\mathrm{dd}$, $J=7.94,4.88 \mathrm{~Hz}, 1 \mathrm{H}) 7.84-7.89(\mathrm{~m}, 1 \mathrm{H}) 8.21(\mathrm{~d}, J=4.88 \mathrm{~Hz}, 1 \mathrm{H}) 8.51(\mathrm{~d}, J=1.22 \mathrm{~Hz}, 1 \mathrm{H})$

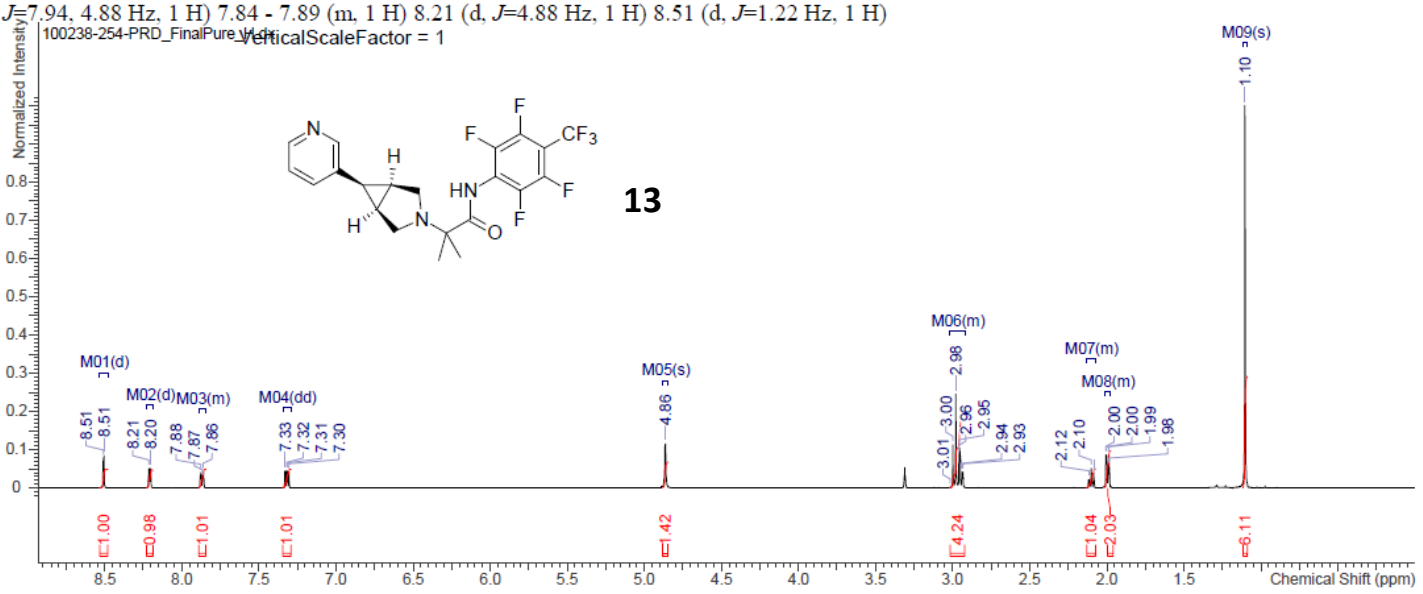

\begin{tabular}{|c|c|c|c|c|}
\hline No. & $(\mathrm{ppm})$ & Value & Absolute Value & Non-Negative Value \\
\hline 1 & {$[1.09 \ldots 1.12]$} & 6.107 & $1.999 \mathrm{e}+9$ & 6.107 \\
\hline 2 & {$[1.98 \ldots 2.01]$} & 2.028 & $6.637 \mathrm{e}+8$ & 2.028 \\
\hline 3 & {$[2.07 \ldots 2.13]$} & 1.038 & $3.398 \mathrm{e}+8$ & 1.038 \\
\hline 4 & {$[2.92 \ldots 3.01]$} & 4.235 & $1.386 \mathrm{e}+9$ & 4.235 \\
\hline 5 & {$[4.85 \ldots 4.88]$} & 1.422 & $4.653 \mathrm{e}+8$ & 1.422 \\
\hline 6 & {$[7.29 .7 .34]$} & 1.010 & $3.307 \mathrm{e}+8$ & 1.010 \\
\hline 7 & {$[7.84 \ldots 7.89]$} & 1.007 & $3.295 \mathrm{e}+8$ & 1.007 \\
\hline 8 & {$[8.19 \ldots 8.23]$} & 0.979 & $3.206 \mathrm{e}+8$ & 0.979 \\
\hline 9 & {$[8.48 . .8 .53]$} & 1.000 & $3.273 \mathrm{e}+8$ & 1.000 \\
\hline
\end{tabular}

5/30/2019 11:27:15 AN

\begin{tabular}{|l|l|}
\hline Multiplets Integrals Sum 0.00 & Number of Nuclei 7 F's \\
\hline
\end{tabular}

\begin{tabular}{|c|c|c|c|c|c|c|c|c|c|}
\hline Acquisition Time (sec) & 0.6990 & Date & \multicolumn{3}{|c|}{$2019 / 03 / 0420: 12: 06+0000$} & Date Stamp & \multicolumn{3}{|c|}{$2019 / 03 / 0420: 12: 06+0000$} \\
\hline Frequency $(\mathrm{MHz})$ & 470.5077 & Nucleus & $19 \mathrm{~F}$ & Number of Transients & 64 & Origin & li_z & Original Points Count & 65536 \\
\hline Owner & li_z & Points Count & 65536 & SW(cyclical) $(\mathrm{Hz})$ & 93751.43 & Solvent & \multicolumn{3}{|c|}{ METHANOL-d4 } \\
\hline Spectrum Offset $(\mathrm{Hz})$ & -56467.046 & & & Spectrum Type & undefined & Sweep Width (Hz) & 93751.43 & Temperature (degree & 25.001 \\
\hline
\end{tabular}

${ }^{19}$ F NMR (471 MHz, METHANOL-d $d_{4}$ $\delta$ ppm -145.02 - -144.83 (m, 2 F) -143.81 - -143.54 (m, 2 F) -57.67 (t, J=21.46 Hz, 3 F)

| 100238-254-PRDManatipure $\backslash$ FefticalScaleFactor $=1$

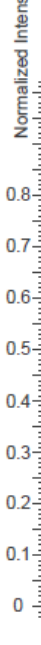

$$
\text { 产 }
$$

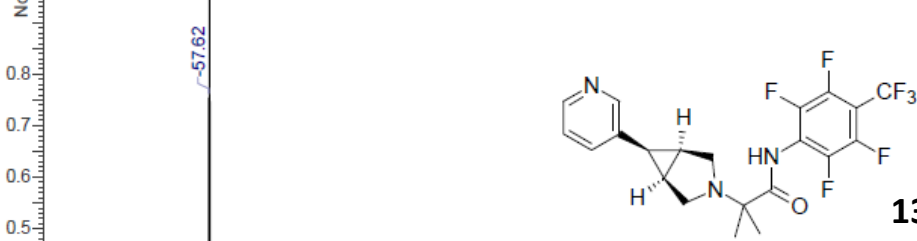

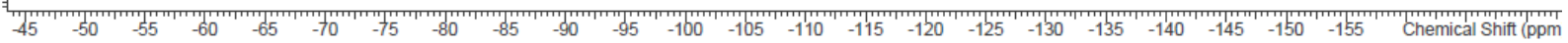

\begin{tabular}{|c|c|c|c|c|c|c|}
\hline No. & $\begin{array}{c}\text { Shift1 } \\
(\mathrm{ppm})\end{array}$ & F's & Type & $\begin{array}{c}\mathrm{J} \\
(\mathrm{Hz})\end{array}$ & Multiplet1 & $(\mathrm{ppm})$ \\
\hline 1 & -144.92 & 2 & $\mathrm{~m}$ & - & M03 & {$[-145.02 \ldots-144.83]$} \\
\hline 2 & -143.67 & 2 & $\mathrm{~m}$ & - & M02 & {$[-143.81 \ldots-143.54]$} \\
\hline 3 & -57.67 & 3 & $\mathrm{t}$ & 21.46 & M01 & {$[-57.77 \ldots-57.58]$}
\end{tabular}




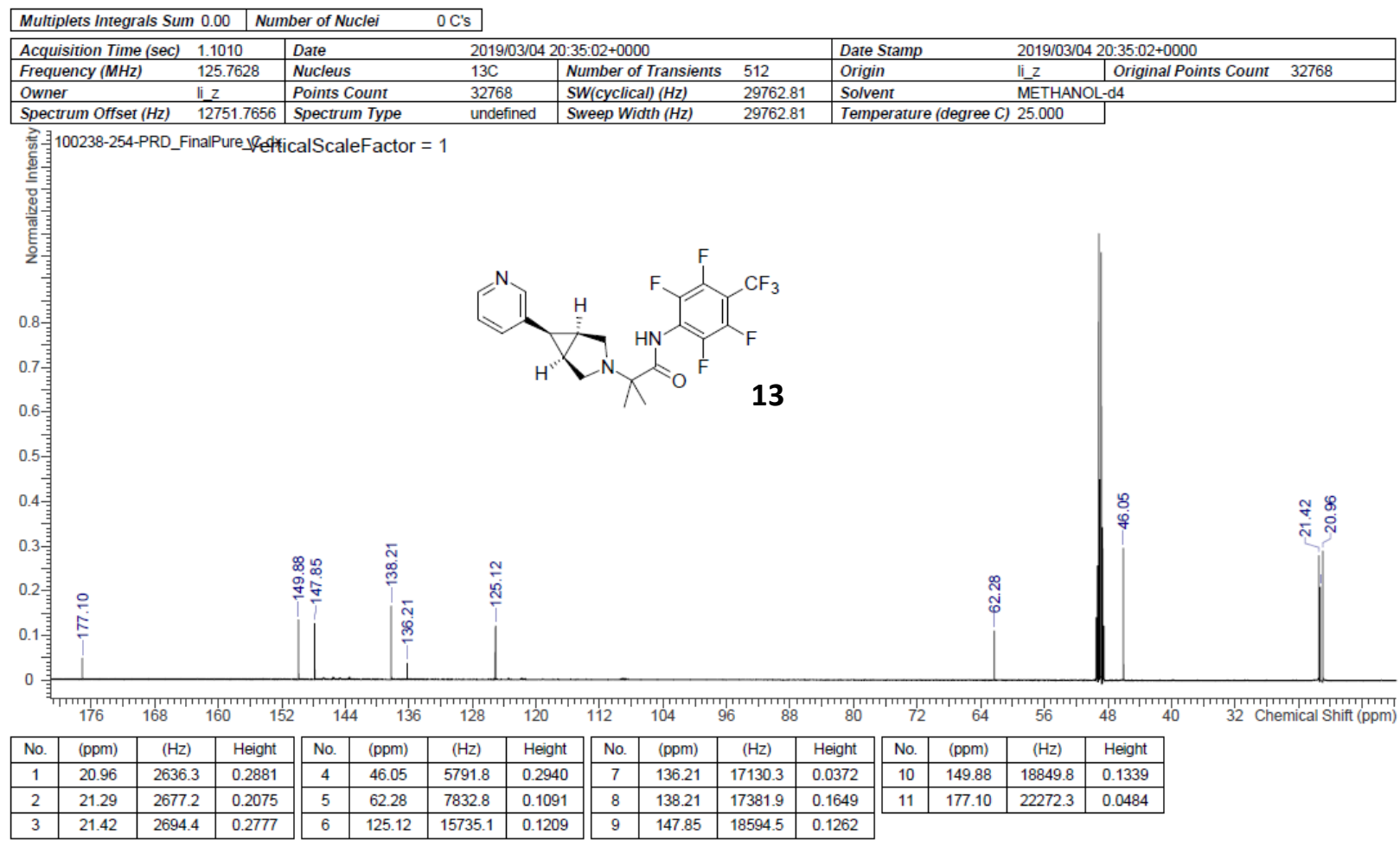

6/2/2019 3:25:59 PM

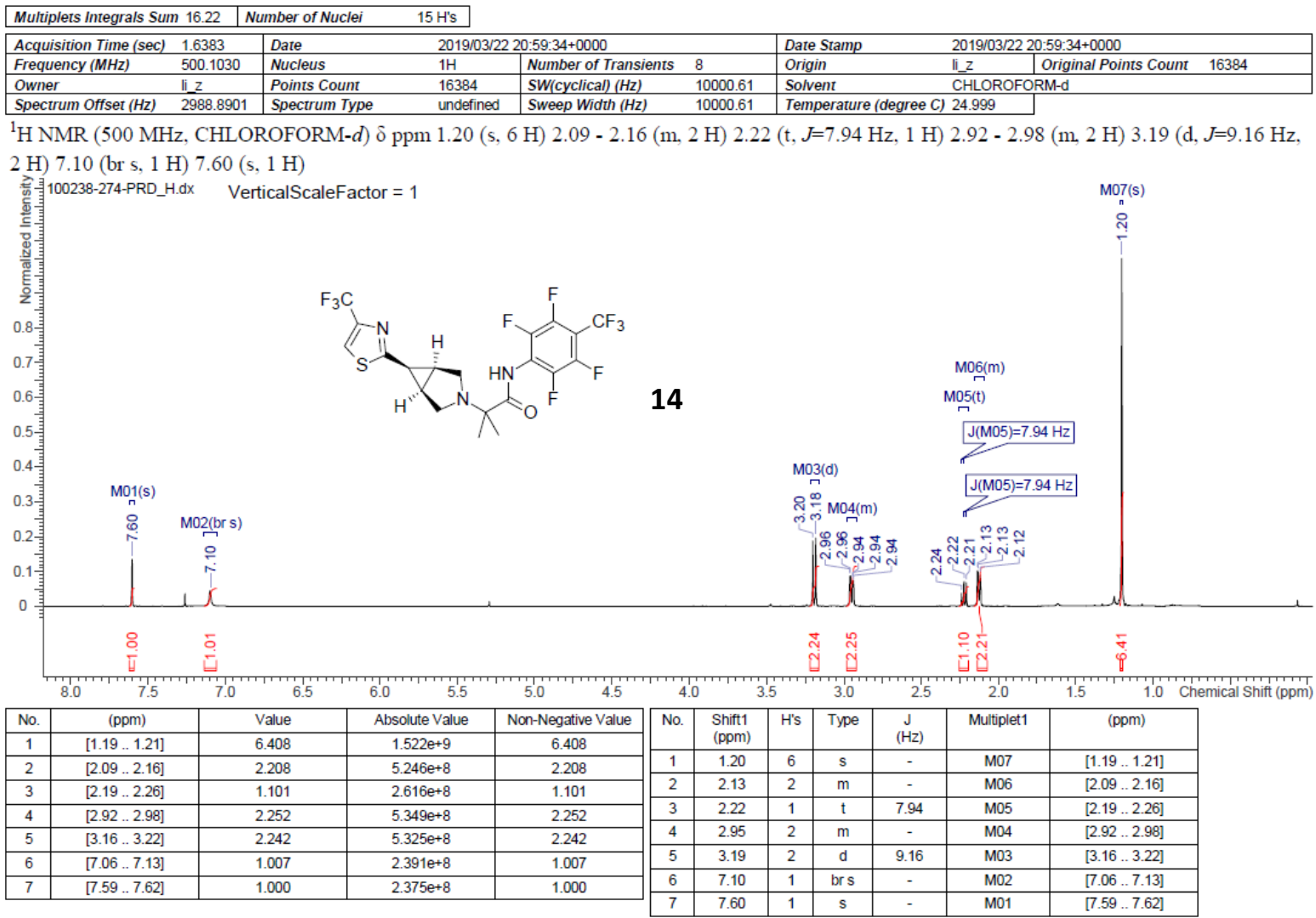




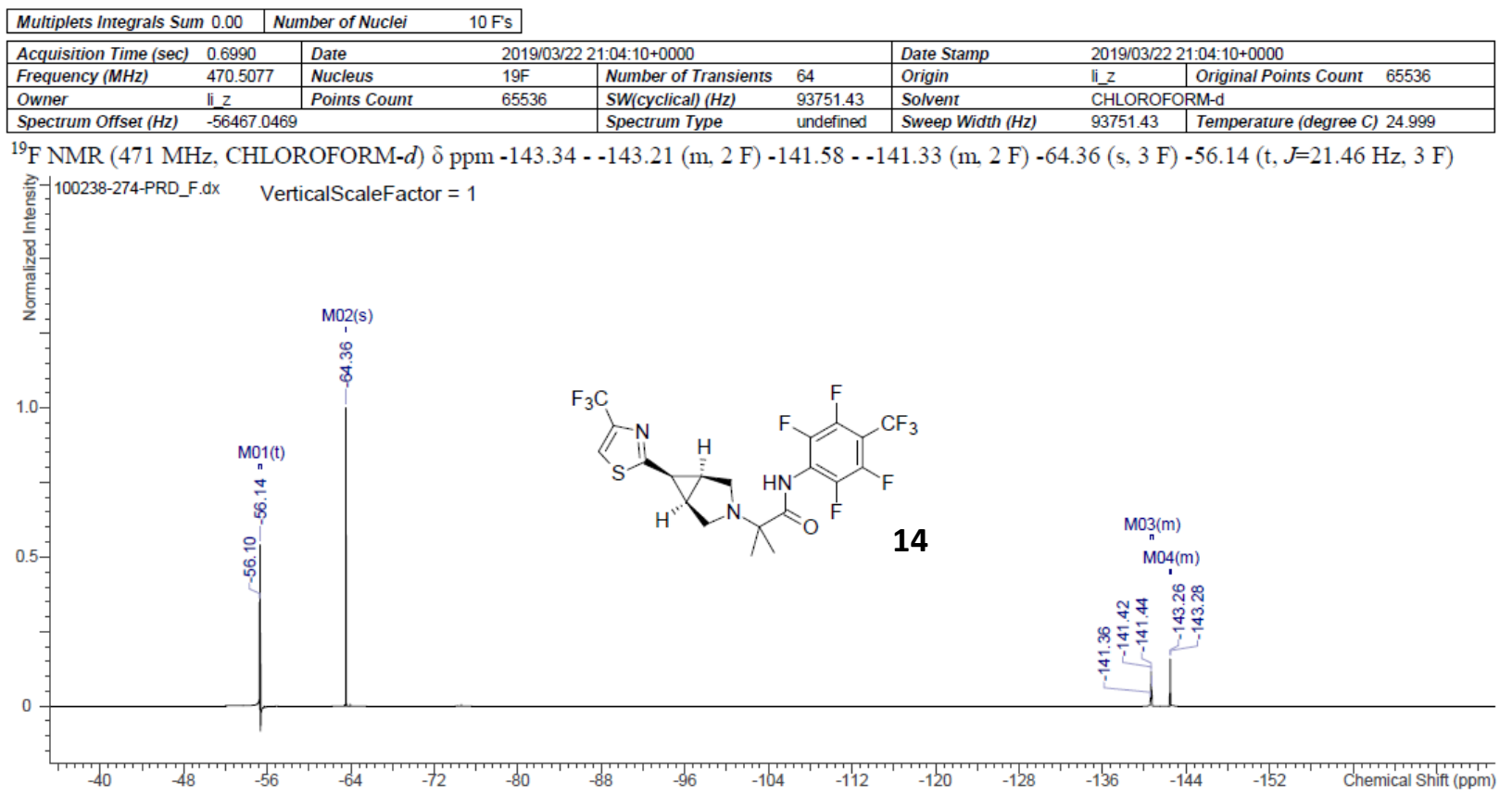

\begin{tabular}{|c|c|c|c|c|c|ccccc|}
\hline No. & $\begin{array}{c}\text { Shift1 } \\
(\mathrm{ppm})\end{array}$ & F's & Type & $\begin{array}{c}\mathrm{J} \\
(\mathrm{Hz})\end{array}$ & Multiplet1 & $\begin{array}{l}-70 \\
(\mathrm{ppm})\end{array}$ \\
\hline 1 & -143.27 & 2 & $\mathrm{~m}$ & - & M04 & {$[-143.34 \ldots-143.21]$} \\
\hline 2 & -141.45 & 2 & $\mathrm{~m}$ & - & M03 & {$[-141.58 \ldots-141.33]$} \\
\hline 3 & -64.36 & 3 & $\mathrm{~s}$ & - & M02 & {$[-64.39 \ldots-64.33]$} \\
\hline 4 & -56.14 & 3 & $\mathrm{t}$ & 21.46 & M01 & {$[-56.32 \ldots-55.99]$} \\
\hline
\end{tabular}

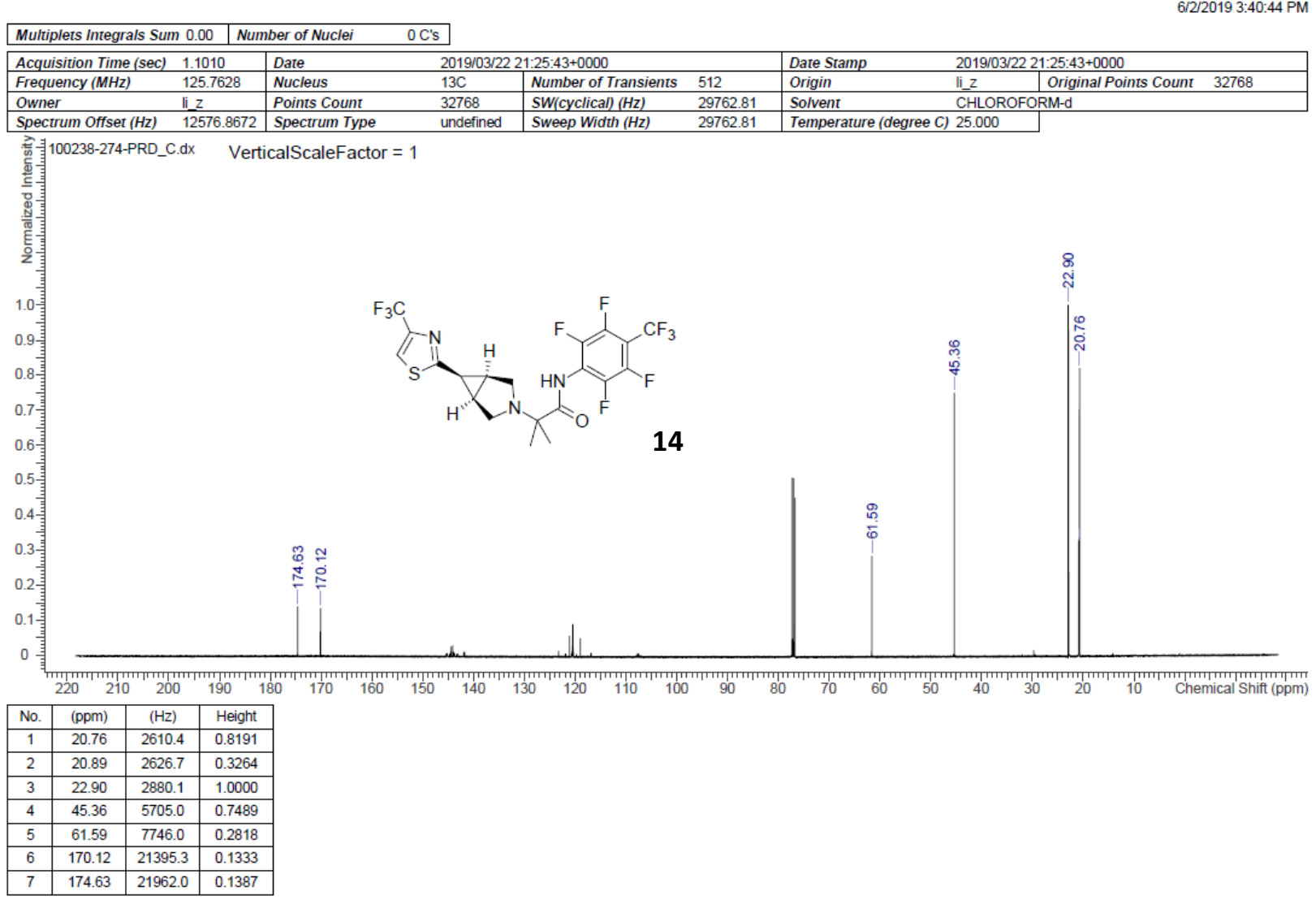




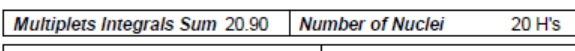

\begin{tabular}{|c|c|c|c|c|c|c|c|c|c|}
\hline Acquisition Time (sec) & 1.6383 & Date & $2019 / 05 / 16$ & $3: 39: 16+0000$ & & Date Stamp & $2019 / 05$ & 3:39:16+0000 & \\
\hline Frequency $(\mathrm{MHz})$ & 500.1030 & Nucleus & $1 \mathrm{H}$ & Number of Transients & 8 & Origin & lizz & Original Points Count & 16384 \\
\hline Owner & li_z & Points Count & 16384 & SW(cyclical) $(\mathrm{Hz})$ & 10000.61 & Solvent & $\mathrm{CHLOR}$ & RM-d & \\
\hline Spectrum Offset $(\mathrm{Hz})$ & 2988.2654 & Spectrum Type & undefined & Sweep Width $(\mathrm{Hz})$ & 10000.61 & Temperature (degree $C$ ) & 25.000 & & \\
\hline
\end{tabular}
\begin{tabular}{|ll|l|ll|l|l|}
\hline Spectrum Offset $(\mathrm{Hz})$ & 2988.2654 & Spectrum Type & undefined & Sweep Width $(\mathrm{Hz})$ & 10000.61 & Temperature (degree C) 25.000 \\
\hline
\end{tabular}

${ }^{1} \mathrm{H}$ NMR (500 MHz, CHLOROFORM-d) $\delta$ ppm 1.12 (s, $\left.6 \mathrm{H}\right) 1.89$ - 1.95 (m, $\left.2 \mathrm{H}\right) 2.18$ (t, J=7.94 Hz, $\left.1 \mathrm{H}\right) 2.65$ (s, 3 H) 2.91 (br d, J=9.16 Hz, 2 H) $3.00(\mathrm{~d}, J=9.16 \mathrm{~Hz}, 2 \mathrm{H}) 6.18$ (br s, $1 \mathrm{H}) 7.43(\mathrm{~d}, J=8.55 \mathrm{~Hz}, 1 \mathrm{H}) 7.71(\mathrm{~s}, 1 \mathrm{H}) 7.75(\mathrm{~d}, J=8.55 \mathrm{~Hz}, 1 \mathrm{H})$

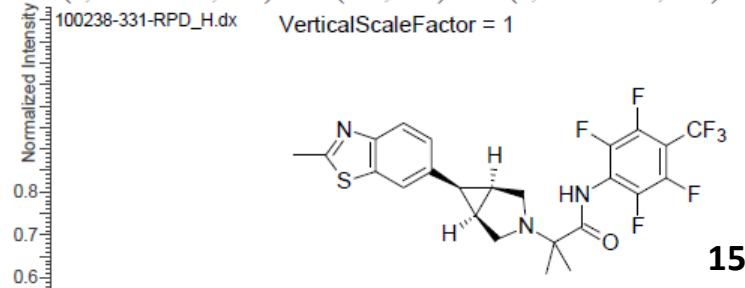

15

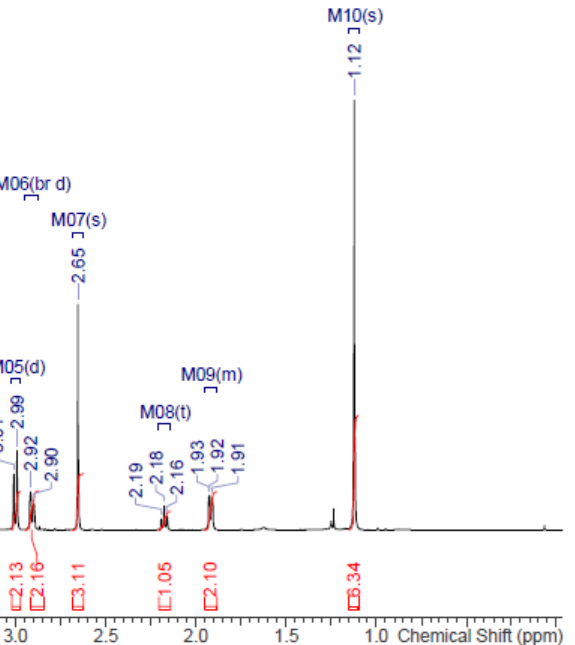

\begin{tabular}{|c|c|c|c|c|c|c|c|c|c|c|c|c|c|}
\hline No. & $\begin{array}{l}\text { Shift11 } \\
\text { (ppm) }\end{array}$ & H's & Type & $\underset{(\mathrm{Hz})}{J}$ & Multiplet1 & (ppm) & No. & $\begin{array}{l}\text { Shift1 } \\
\text { (ppm) }\end{array}$ & H's & Type & $\underset{(\mathrm{Hz})}{\mathrm{J}}$ & Multiplet1 & (ppm) \\
\hline 1 & 1.12 & 6 & s & - & M10 & {$\left[\begin{array}{llll}1.10 & \ldots & 1.15]\end{array}\right.$} & 6 & 3.00 & 2 & d & 9.16 & M05 & {$[2.97 \ldots 3.02]$} \\
\hline 2 & 1.92 & 2 & $\mathrm{~m}$ & - & M09 & {$\left[\begin{array}{llll}1.89 & \ldots & 1.95]\end{array}\right.$} & 7 & 6.18 & 1 & brs & - & M04 & {$[6.13 \ldots 6.21]$} \\
\hline 3 & 2.18 & 1 & $t$ & 7.94 & M08 & {$\left[\begin{array}{llll}2.14 & .2 .21\end{array}\right]$} & 8 & 7.43 & 1 & d & 8.55 & M03 & {$[7.40 \ldots 7.45]$} \\
\hline 4 & 2.65 & 3 & $\mathrm{~s}$ & - & M07 & {$\left[\begin{array}{llll}2.62 & . .2 & 2.68\end{array}\right]$} & 9 & 7.71 & 1 & $\mathrm{~s}$ & - & M02 & {$[7.69 \ldots .7 .72]$} \\
\hline 5 & 2.91 & 2 & brd & 9.16 & M06 & {$\left[\begin{array}{llll}2.87 & .2 & 2.95\end{array}\right]$} & 10 & 7.75 & 1 & d & 8.55 & M01 & [7.73 .. 7.77] \\
\hline
\end{tabular}

\begin{tabular}{|l|l|}
\hline Multiplets Integrals Sum 0.00 & Number of Nuclei 7 F's \\
\hline
\end{tabular}

\begin{tabular}{|c|c|c|c|c|c|c|c|c|}
\hline Acquisition Time (sec) & 0.6990 & Date & \multicolumn{3}{|c|}{ 2019/05/16 13:43:02+0000 } & Date Stamp & \multicolumn{2}{|l|}{$2019 / 05 / 16$ 13:43:02+0000 } \\
\hline Frequency $(\mathrm{MHz})$ & 470.5077 & Nucleus & $19 \mathrm{~F}$ & Number of Transients & 64 & Origin & \begin{tabular}{l|l} 
li_z & Original Points Count
\end{tabular} & 65536 \\
\hline Owner & li_z & Points Count & 65536 & SW(cyclical) $(\mathrm{Hz})$ & 93751.43 & Solvent & HLOROFORM-d & \\
\hline
\end{tabular}

${ }^{19} \mathrm{~F}$ NMR (471 MHz, CHLOROFORM- $d$ ) $\delta \mathrm{ppm}-143.04--142.87$ (m, 2 F) -141.66 - -141.38 (m, 2 F) -55.89 (t, J=21.46 Hz, 3 F)
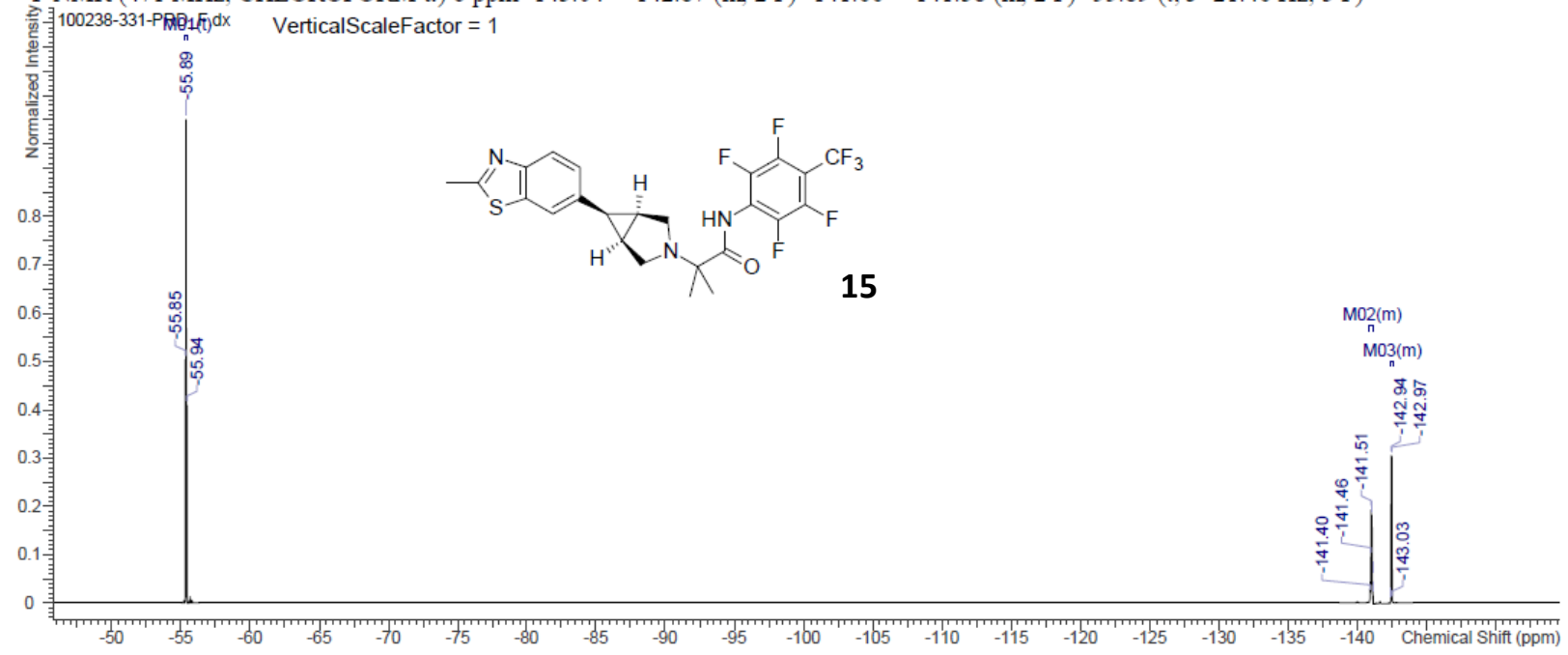

\begin{tabular}{|c|c|c|c|c|c|c|}
\hline No. & $\begin{array}{c}\text { Shift1 } \\
(\mathrm{ppm})\end{array}$ & F's & Type & $\begin{array}{c}\mathrm{J} \\
(\mathrm{Hz})\end{array}$ & Multiplet1 & $(\mathrm{ppm})$ \\
\hline 1 & -142.95 & 2 & $\mathrm{~m}$ & - & $\mathrm{M} 03$ & {$[-143.04 \ldots-142.87]$} \\
\hline 2 & -141.52 & 2 & $\mathrm{~m}$ & - & $\mathrm{M} 02$ & {$[-141.66 \ldots-141.38]$} \\
\hline 3 & -55.89 & 3 & $\mathrm{t}$ & 21.46 & M01 & {$[-55.98 \ldots-55.81]$} \\
\hline
\end{tabular}


\begin{tabular}{|l|ll|}
\hline Multiplets Integrals Sum 0.00 & Number of Nuclei & $0 \mathrm{C} ' \mathrm{~s}$ \\
\hline
\end{tabular}

\begin{tabular}{|c|c|c|c|c|c|c|c|c|c|}
\hline Acquisition Time (sec) & 1.1010 & Date & $2019 / 05 / 16$ & $4: 24: 15+0000$ & & Date Stamp & $2019 / 05$ & $4: 24: 15+0000$ & \\
\hline Frequency $(\mathrm{MHz})$ & 125.7628 & Nucleus & $13 \mathrm{C}$ & Number of Transients & 32 & Origin & iliz & Original Points Count & 32768 \\
\hline Owner & li_z & Points Count & 32768 & SW(cyclical) $(\mathrm{Hz})$ & 29762.81 & Solvent & CHLOR & $\mathrm{RM}-\mathrm{d}$ & \\
\hline Spectrum Offset (Hz) & 12587.3057 & Spectrum Type & undefined & Sweep Width $(\mathrm{Hz})$ & 29762.81 & Temperature (degree $\mathrm{C}$ ) & 25.001 & & \\
\hline
\end{tabular}

\begin{tabular}{|lll|ll|l|l|}
\hline Spectrum Offset $(\mathrm{Hz})$ & 12587.3057 & Spectrum Type & undefined & Sweep Width $(\mathrm{Hz})$ & 29762.81 & Temperature (degree C) 25.001 \\
\hline
\end{tabular}
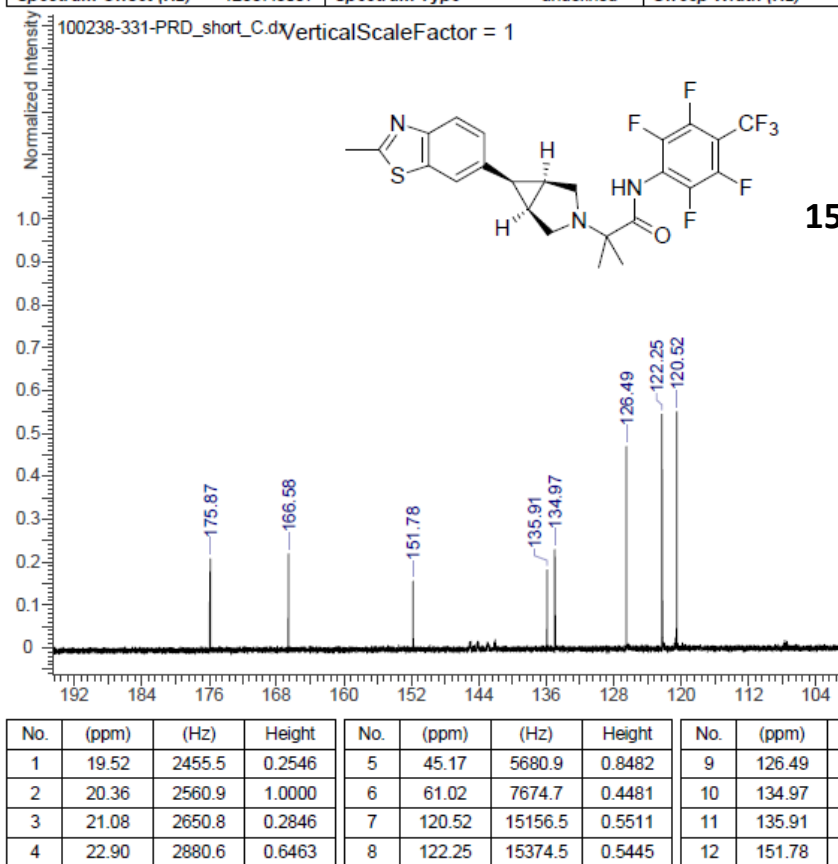

15

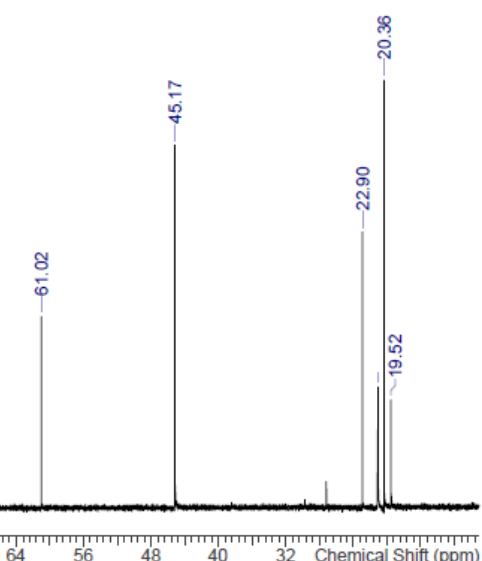

\begin{tabular}{|l|ll|}
\hline Multiplets Integrals Sum 21.49 & Number of Nuclei & $21 \mathrm{H}$ 's \\
\hline
\end{tabular}

\begin{tabular}{|c|c|c|c|c|c|c|c|c|c|}
\hline Acquisition Time (sec) & 1.6383 & \begin{tabular}{|l|} 
Date \\
\end{tabular} & \multicolumn{3}{|c|}{$2019 / 04 / 2319: 32: 29+0000$} & Date Stamp & \multicolumn{3}{|c|}{$2019 / 04 / 2319: 32: 29+0000$} \\
\hline Frequency $(\mathrm{MHz})$ & 500.1030 & Nucleus & $1 \mathrm{H}$ & Number of Transients & 8 & Origin & li_z & Original Points Count & 16384 \\
\hline Owner & lijz & Points Count & 16384 & SW(cyclical) $(\mathrm{Hz})$ & 10000.61 & Solvent & \multicolumn{3}{|c|}{ CHLOROFORM-d } \\
\hline Spectrum Offset $(\mathrm{Hz})$ & 2988.2554 & Spectrum Type & undefined & Sweep Width (Hz) & 10000.61 & Temperature (degree C) & 24.998 & & \\
\hline
\end{tabular}

${ }^{1} \mathrm{H}$ NMR $(500 \mathrm{MHz}, \mathrm{CHLOROFORM}-d) \delta \mathrm{ppm} 1.11$ (s, $\left.6 \mathrm{H}\right) 2.06(\mathrm{~s}, 3 \mathrm{H}) 2.89$ (br d, J=9.16 Hz, $\left.2 \mathrm{H}\right) 3.15$ (d, J=8.55 Hz, $\left.2 \mathrm{H}\right) 3.97$ (s, $\left.3 \mathrm{H}\right) 6.45$ (br s, $1 \mathrm{H}) 6.82-6.89(\mathrm{~m}, 2 \mathrm{H}) 7.05(\mathrm{~d}, J=7.33 \mathrm{~Hz}, 1 \mathrm{H}) 7.70(\mathrm{~d}, J=7.33 \mathrm{~Hz}, 1 \mathrm{H})$

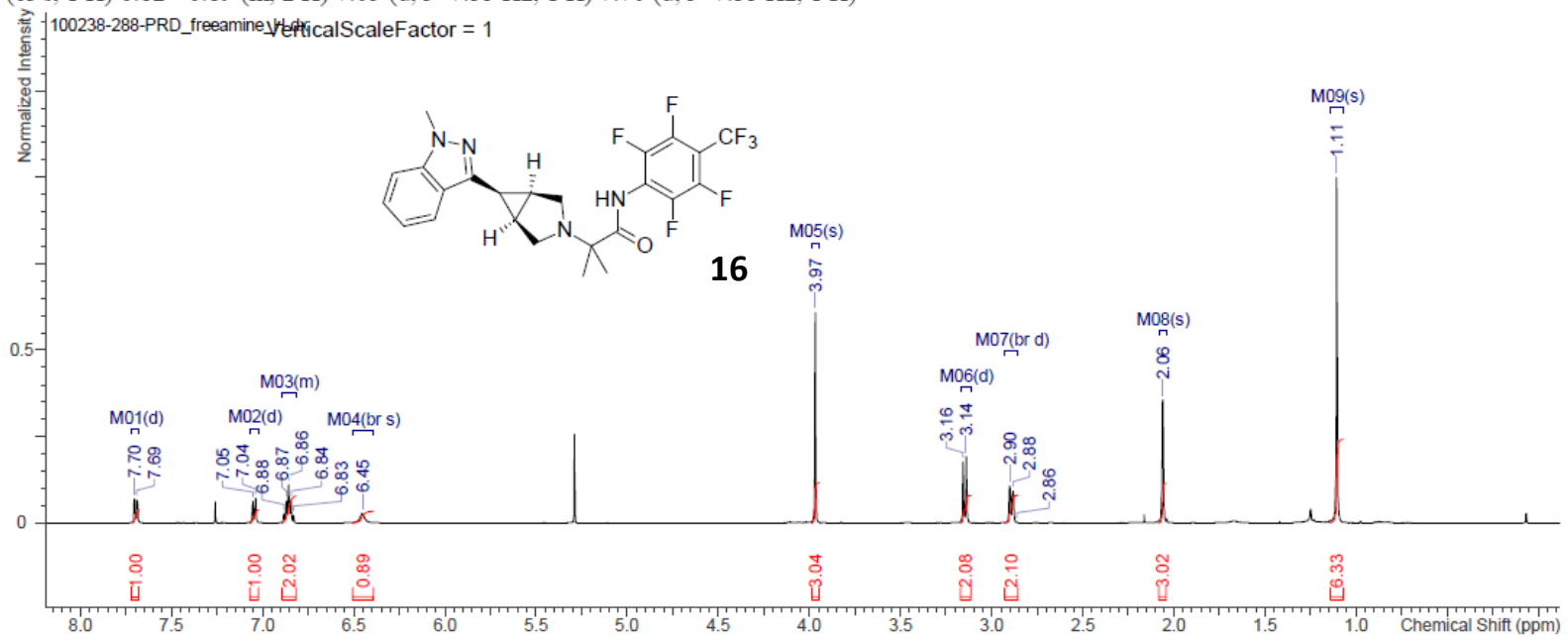

\begin{tabular}{|c|c|c|c|c|}
\hline No. & $(\mathrm{ppm})$ & Value & Absolute Value & Non-Negative Value \\
\hline 1 & {$[1.07 \ldots 1.14]$} & 6.335 & $1.375 \mathrm{e}+9$ & 6.335 \\
\hline 2 & {$[2.04 \ldots 2.08]$} & 3.024 & $6.562 \mathrm{e}+8$ & 3.024 \\
\hline 3 & {$[2.86 \ldots 2.93]$} & 2.099 & $4.556 \mathrm{e}+8$ & 2.099 \\
\hline 4 & {$[3.12 . .3 .17]$} & 2.084 & $4.524 \mathrm{e}+8$ & 2.084 \\
\hline 5 & {$[3.95 \ldots 3.99]$} & 3.039 & $6.595 \mathrm{e}+8$ & 3.039 \\
\hline 6 & {$[6.39 . .6 .51]$} & 0.893 & $1.938 \mathrm{e}+8$ & 0.893 \\
\hline 7 & {$[6.82 \ldots 6.89]$} & 2.021 & $4.387 \mathrm{e}+8$ & 2.021 \\
\hline 8 & {$[7.02 . .7 .07]$} & 0.996 & $2.163 \mathrm{e}+8$ & 0.996 \\
\hline 9 & {$[7.68 \ldots 7.72]$} & 1.000 & $2.170 \mathrm{e}+8$ & 1.000 \\
\hline
\end{tabular}


\begin{tabular}{|l|l|}
\hline Multiplets Integrals Sum 0.00 & Number of Nuclei 7 F's \\
\hline
\end{tabular}

\begin{tabular}{|c|c|c|c|c|c|c|c|c|c|}
\hline Acquisition Time (sec) & 0.6990 & Date & \multicolumn{3}{|c|}{$2019 / 04 / 23$ 19:36:31+0000 } & Date Stamp & \multicolumn{3}{|c|}{$2019 / 04 / 23$ 19:36:31+0000 } \\
\hline Frequency $(\mathrm{MHz})$ & 470.5077 & Nucleus & $19 \mathrm{~F}$ & Number of Transients & 64 & Origin & li_z & Original Points Count & 65536 \\
\hline Owner & li_z & Points Count & 65536 & SW(cyclical) $(\mathrm{Hz})$ & 93751.43 & Solvent & \multicolumn{3}{|c|}{ CHLOROFORM-d } \\
\hline Spectrum Offset $(\mathrm{Hz})$ & -56467.046 & & & Spectrum Type & undefined & Sweep Width $(\mathrm{Hz})$ & 93751.43 & Temperature (degree $\mathrm{C}$ ) & 25.000 \\
\hline
\end{tabular}

\begin{tabular}{|ll|ll|lll|l}
\hline Spectrum Offset $(\mathrm{Hz})$ & -56467.0469 & Spectrum Type & undefined & Sweep Width $(\mathrm{Hz})$ & 93751.43 & Temperature $($ degree C) 25.000 \\
\hline
\end{tabular}

${ }^{19} \mathrm{~F}$ NMR (471 MHz, CHLOROFORM- $d$ ) $\delta$ ppm -143.77 - -143.58 (m, 2 F) -142.42 - -142.10 (m, 2 F) -56.10 (t, J=21.46 Hz, 3 F)

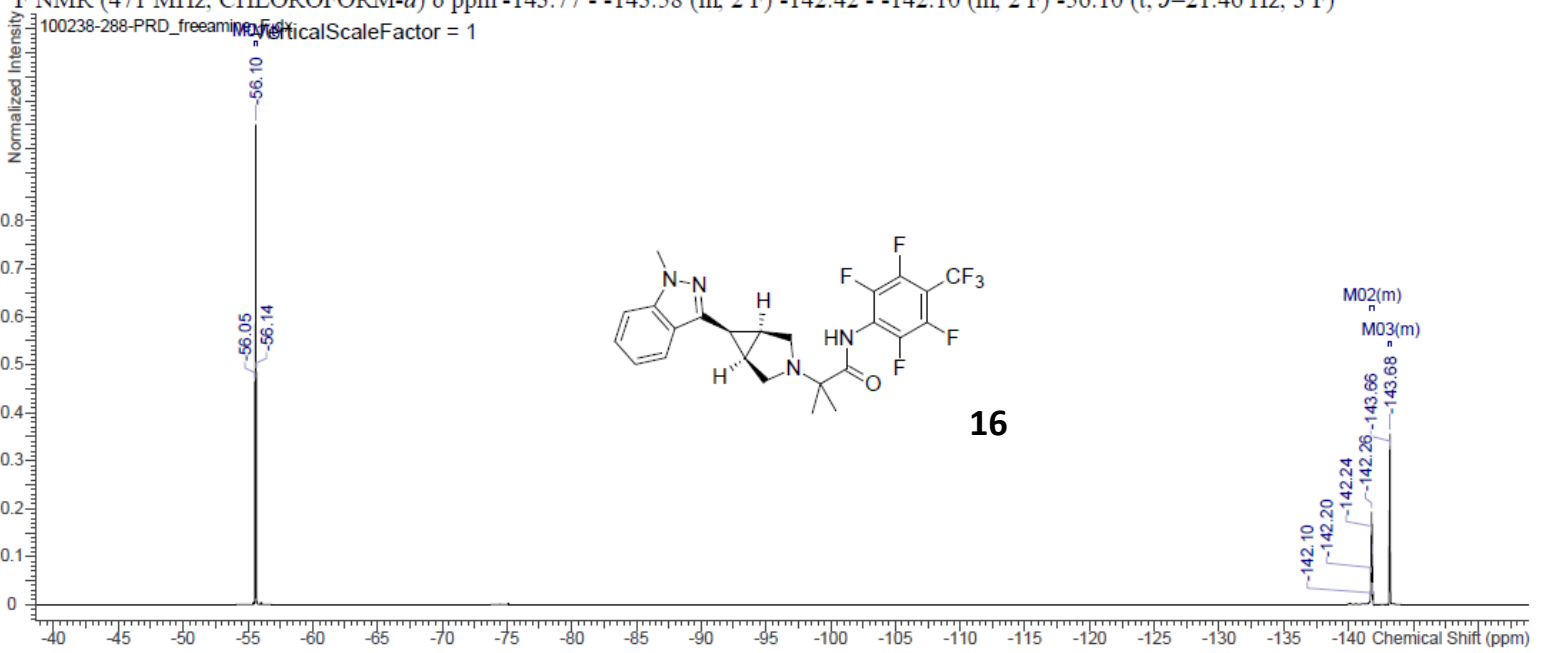

\begin{tabular}{|c|c|c|c|c|c|c|}
\hline No. & $\begin{array}{c}\text { Shift1 } \\
(\mathrm{ppm})\end{array}$ & F's & Type & $\begin{array}{c}J \\
(\mathrm{~Hz})\end{array}$ & Multiplet1 & $(\mathrm{ppm})$ \\
\hline 1 & -143.67 & 2 & $\mathrm{~m}$ & - & M03 & {$[-143.77 \ldots-143.58]$} \\
\hline 2 & -142.27 & 2 & $\mathrm{~m}$ & - & M02 & {$[-142.42 \ldots-142.10]$} \\
\hline 3 & -56.10 & 3 & $\mathrm{t}$ & 21.46 & M01 & {$[-56.24 \ldots-56.01]$} \\
\hline
\end{tabular}

\begin{tabular}{|l|ll|}
\hline Multiplets Integrals Sum 0.00 & Number of Nuclei & 0 C's \\
\hline
\end{tabular}

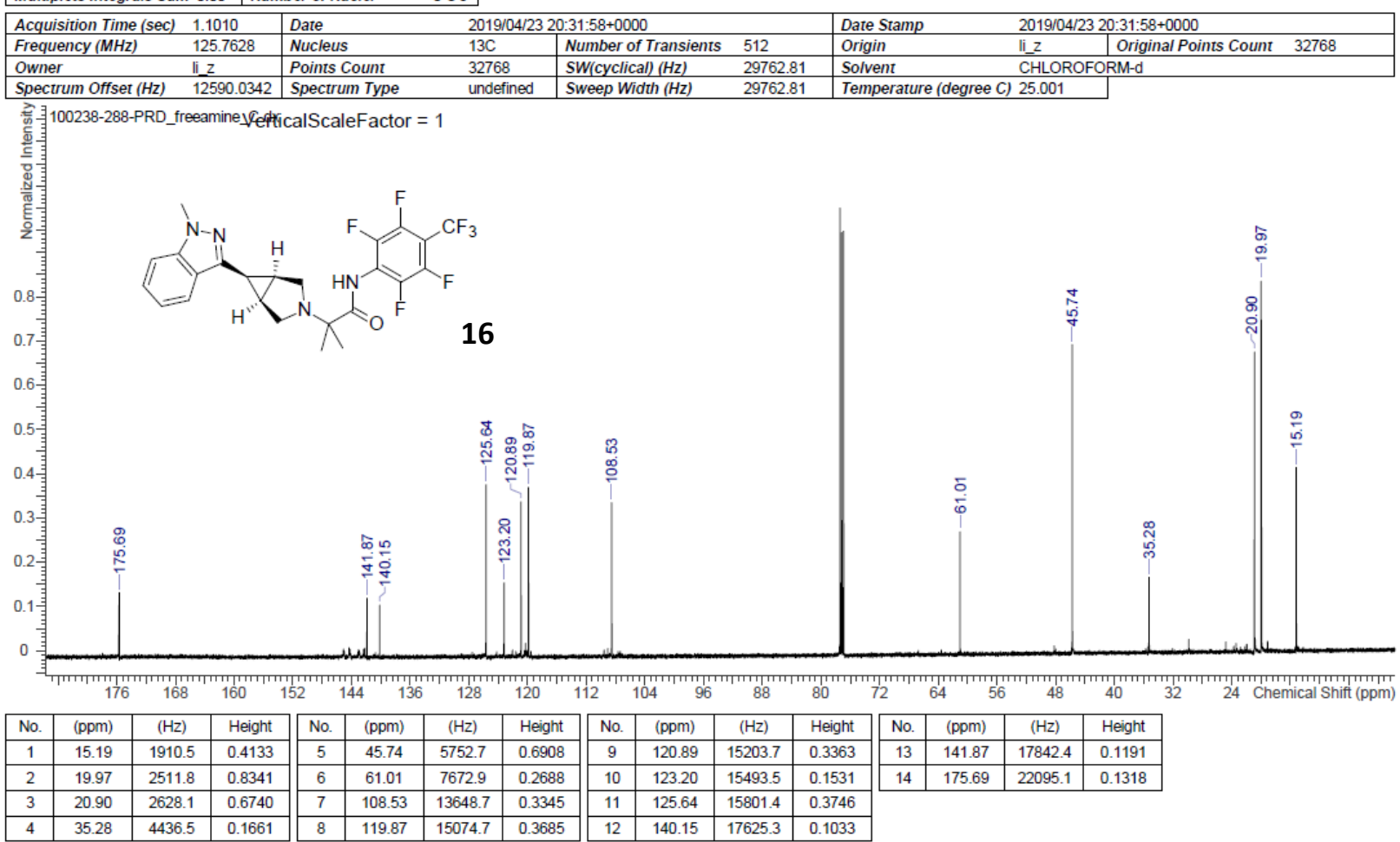




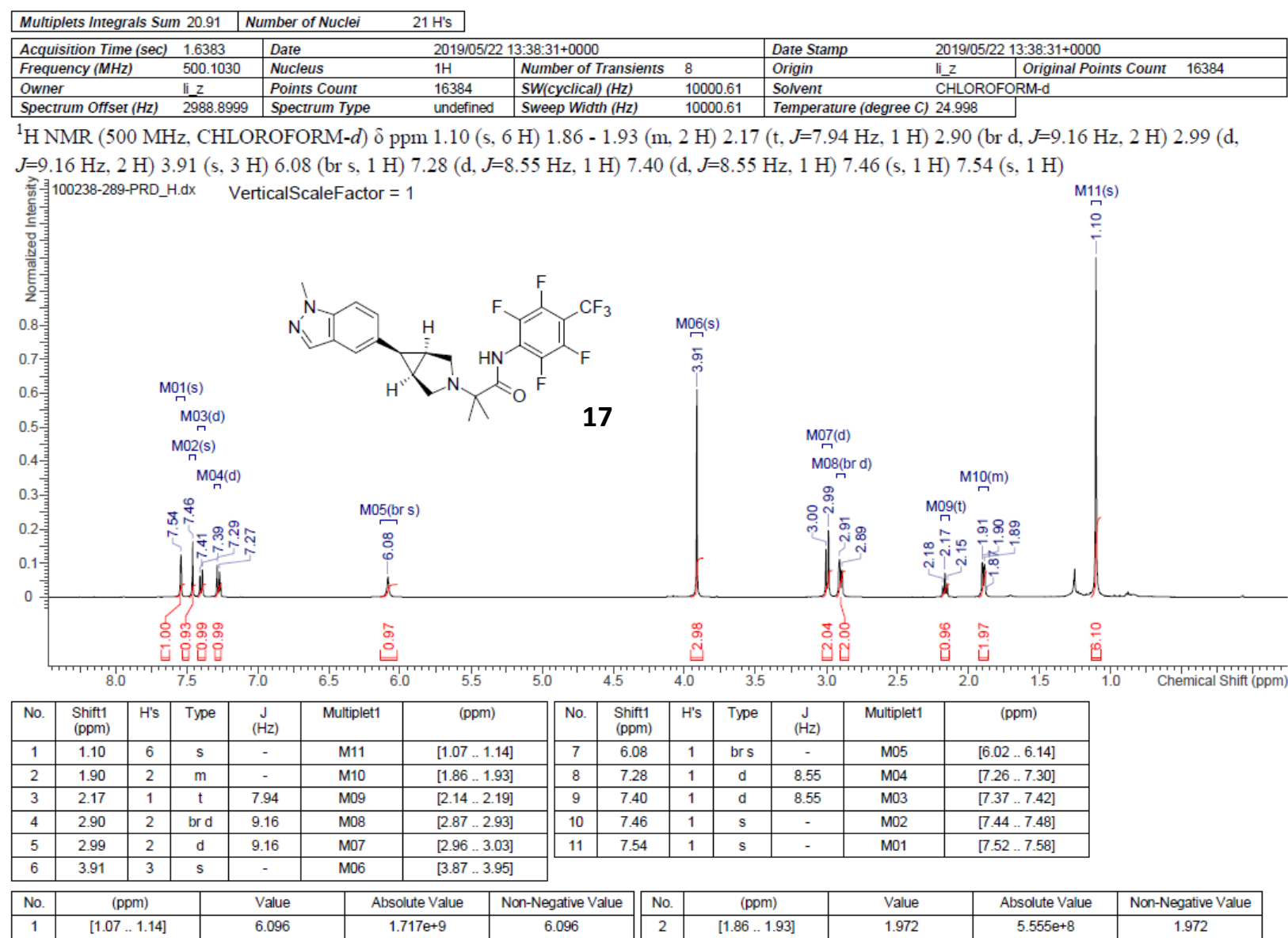

\begin{tabular}{|c|c|c|c|c|c|c|c|c|c|}
\hline Multiplets Integrals Sun & 0.00 & ber of Nuclei & $7 \mathrm{Fs}$ & & & & & & \\
\hline Acquisition Time (sec) & 0.6990 & Date & $2019 / 0$ & $3: 42: 10+0000$ & & Date Stamp & $2019 / 05 / 2$ & $3: 42: 10+0000$ & \\
\hline Frequency $(\mathrm{MHz})$ & 470.5077 & Nucleus & $19 \mathrm{~F}$ & Number of Transients & 64 & Origin & li_z & Original Points Count & 65536 \\
\hline Owner & li_z & Points Count & 65536 & $S W$ (cyclical) $(\mathrm{Hz})$ & 93751.43 & Solvent & CHLOROH & RM-d & \\
\hline Spectrum Offset $(\mathrm{Hz})$ & -56467.0 & & & Spectrum Type & undefined & Sweep Width $(\mathrm{Hz})$ & 93751.43 & \begin{tabular}{|l|l} 
Temperature (degree $\mathrm{C}$ ) \\
(a)
\end{tabular} & 25.001 \\
\hline
\end{tabular}

${ }^{19}$ F NMR (471 MHz, CHLOROFORM-d) $\delta$ ppm -144.63 - -144.29 (m. 2 F) -142.07 - -141.78 (m. 2 F) -56.17 (t. J=21.46 Hz, 3 F)

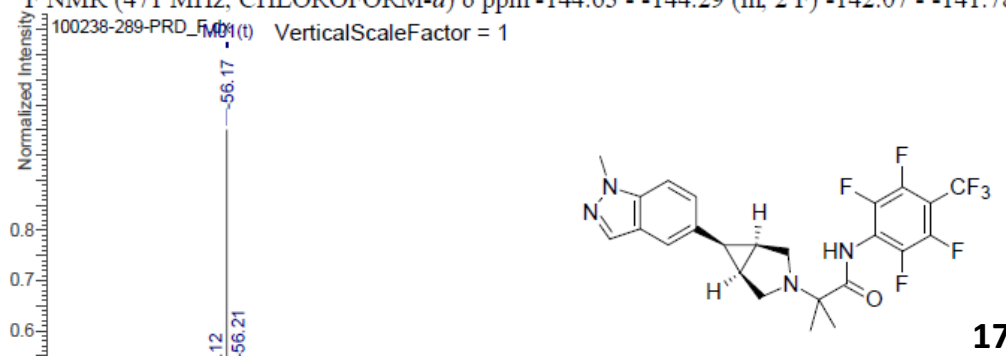

17

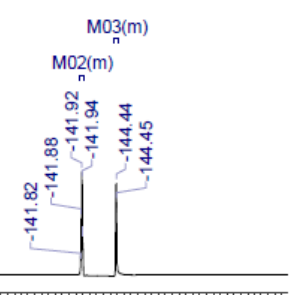

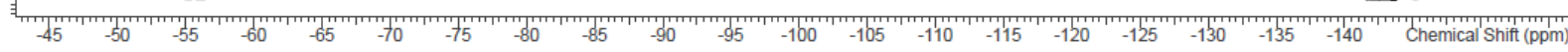

\begin{tabular}{|c|c|c|c|c|c|c|}
\hline No. & $\begin{array}{c}\text { Shift1 } \\
(\mathrm{ppm})\end{array}$ & $\mathrm{F}$ 's & Type & $\begin{array}{c}\mathrm{J} \\
(\mathrm{Hz})\end{array}$ & Multiplet1 & $(\mathrm{ppm})$ \\
\hline 1 & -144.43 & 2 & $\mathrm{~m}$ & - & $\mathrm{M} 03$ & {$[-144.63 \ldots-144.29]$} \\
\hline 2 & -141.93 & 2 & $\mathrm{~m}$ & - & M02 & {$[-142.07 \ldots-141.78]$} \\
\hline 3 & -56.17 & 3 & $\mathrm{t}$ & 21.46 & M01 & {$[-56.25 \ldots-56.09]$} \\
\hline
\end{tabular}




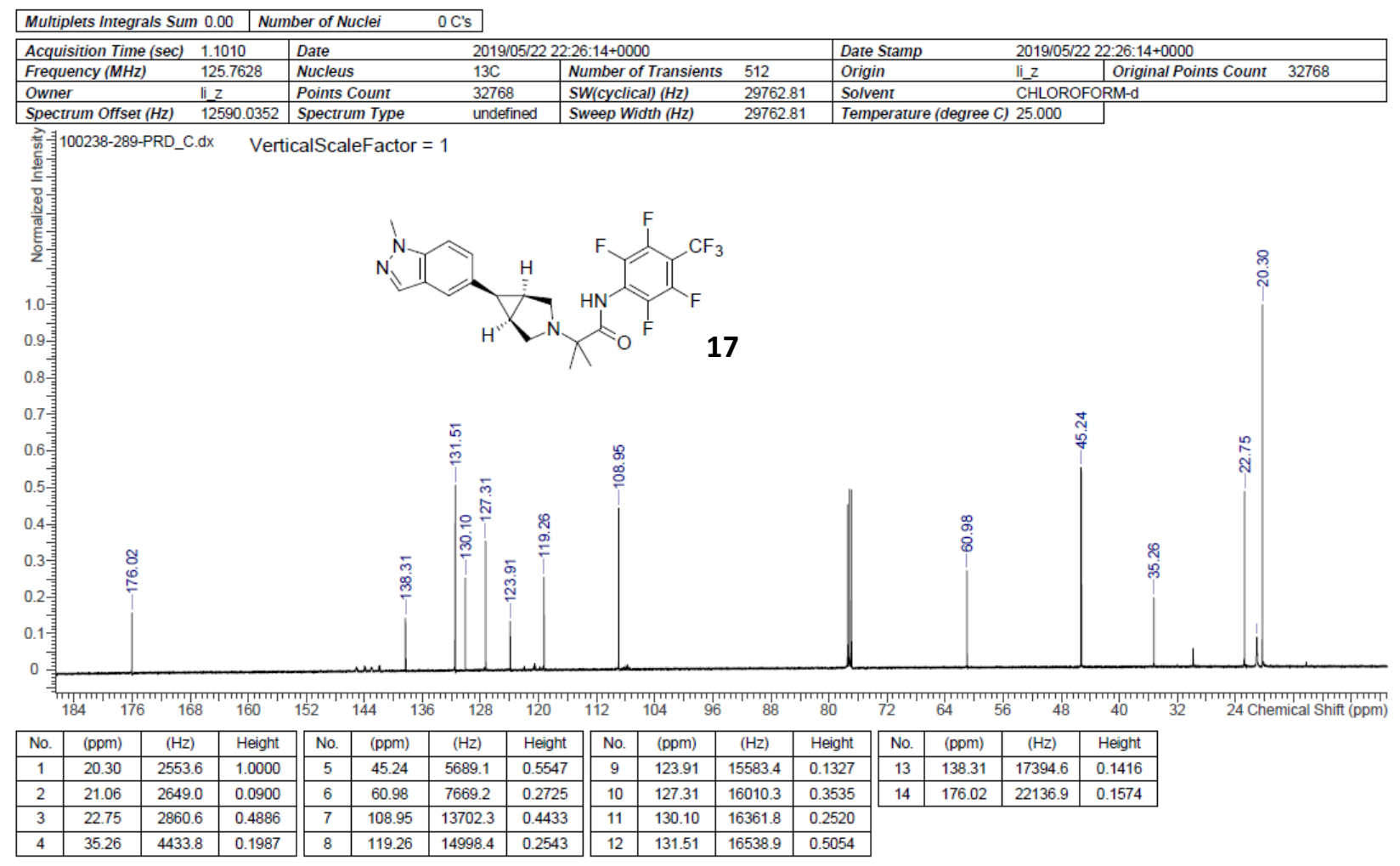

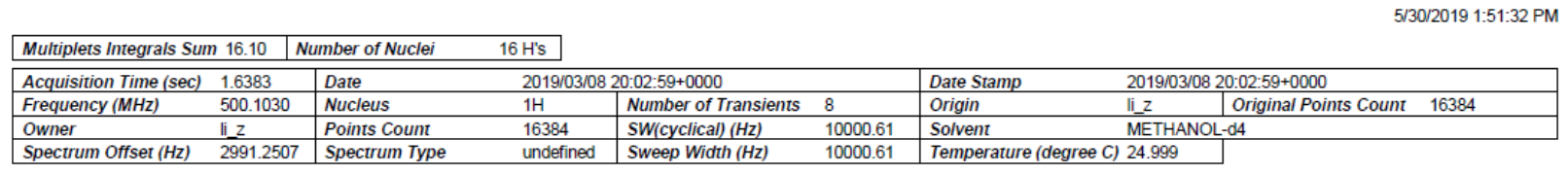

${ }^{1} \mathrm{H}$ NMR $\left(500 \mathrm{MHz}\right.$, METHANOL- $\left.d_{4}\right) \delta \mathrm{ppm} 1.09(\mathrm{~s}, 6 \mathrm{H}) 2.00$ - $2.10(\mathrm{~m}, 3 \mathrm{H}) 2.96-3.02(\mathrm{~m}, 4 \mathrm{H}) 8.79(\mathrm{~s}, 2 \mathrm{H}) 8.91(\mathrm{~s}, 1 \mathrm{H})$

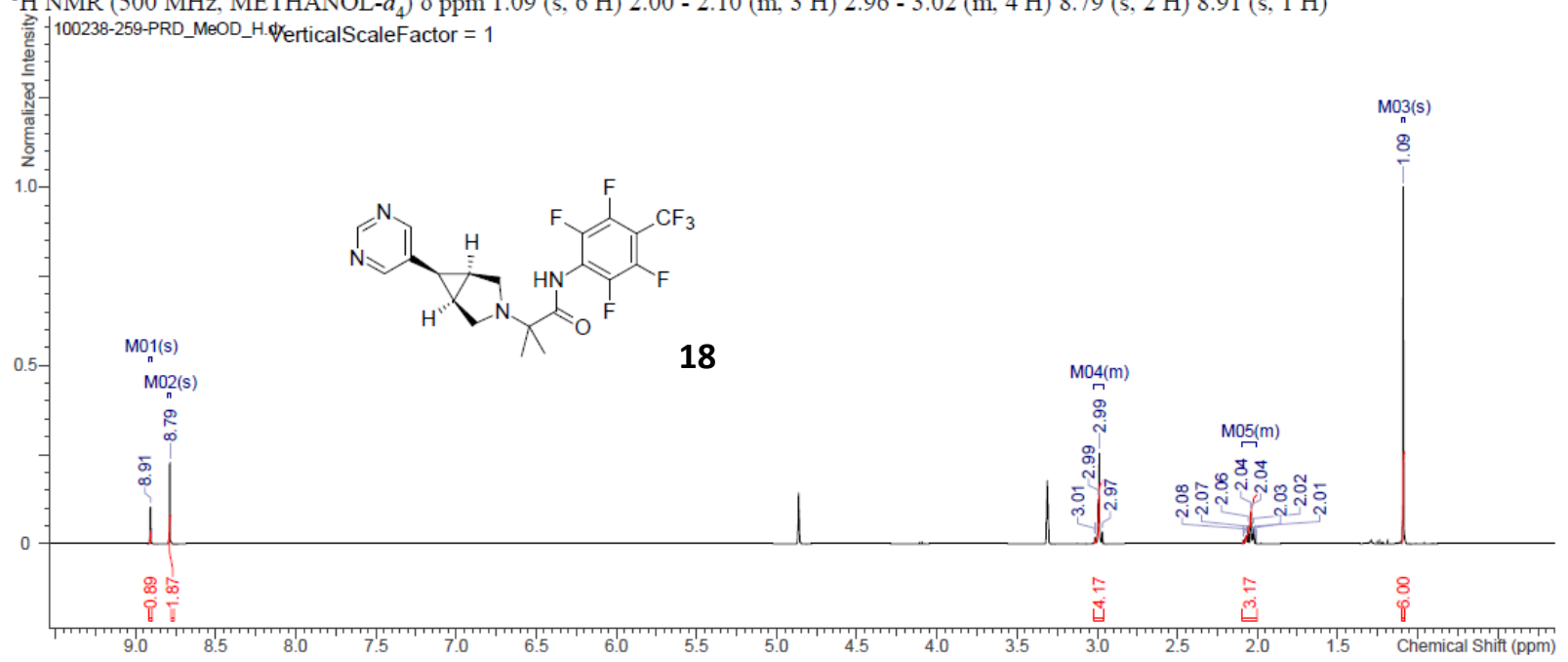

\begin{tabular}{|c|c|c|c|c|c|c|c|c|c|c|}
\hline & & & & & \multirow{2}{*}{ No. } & \multirow{2}{*}{$\begin{array}{l}\text { Shift1 } \\
(\mathrm{ppm})\end{array}$} & \multirow{2}{*}{ H's } & \multirow{2}{*}{ Type } & \multirow{2}{*}{ Multiplet1 } & \multirow{2}{*}{ (ppm) } \\
\hline 1 & [1.08 . . 1.10] & 6.000 & $6.495 \mathrm{e}+8$ & 6.000 & & & & & & \\
\hline 2 & {$[2.00 \ldots 2.09]$} & 3.168 & $3.430 \mathrm{e}+8$ & 3.168 & 1 & 1.09 & 6 & $s$ & M03 & [1.08 .. 1.10] \\
\hline 3 & {$\left[\begin{array}{llll}2.96 & .3 .02]\end{array}\right]$} & 4.165 & $4.509 \mathrm{e}+8$ & 4.165 & 2 & 2.04 & 3 & $\mathrm{~m}$ & M05 & {$[2.00 \ldots 2.10]$} \\
\hline 4 & {$[8.78 \ldots .8 .80]$} & 1.874 & $2.028 \mathrm{e}+8$ & 1.874 & 3 & 2.99 & 4 & $\mathrm{~m}$ & M04 & {$\left[\begin{array}{lll}2.96 & \ldots & 3.02]\end{array}\right]$} \\
\hline 5 & {$[8.90 \ldots .8 .92]$} & 0.888 & $9.617 \mathrm{e}+7$ & 0.888 & 4 & 8.79 & 2 & $s$ & M02 & [8.78 $\ldots .8 .80]$ \\
\hline & & & & & 5 & 8.91 & 1 & s & M01 & [8.90 .. 8.92] \\
\hline
\end{tabular}



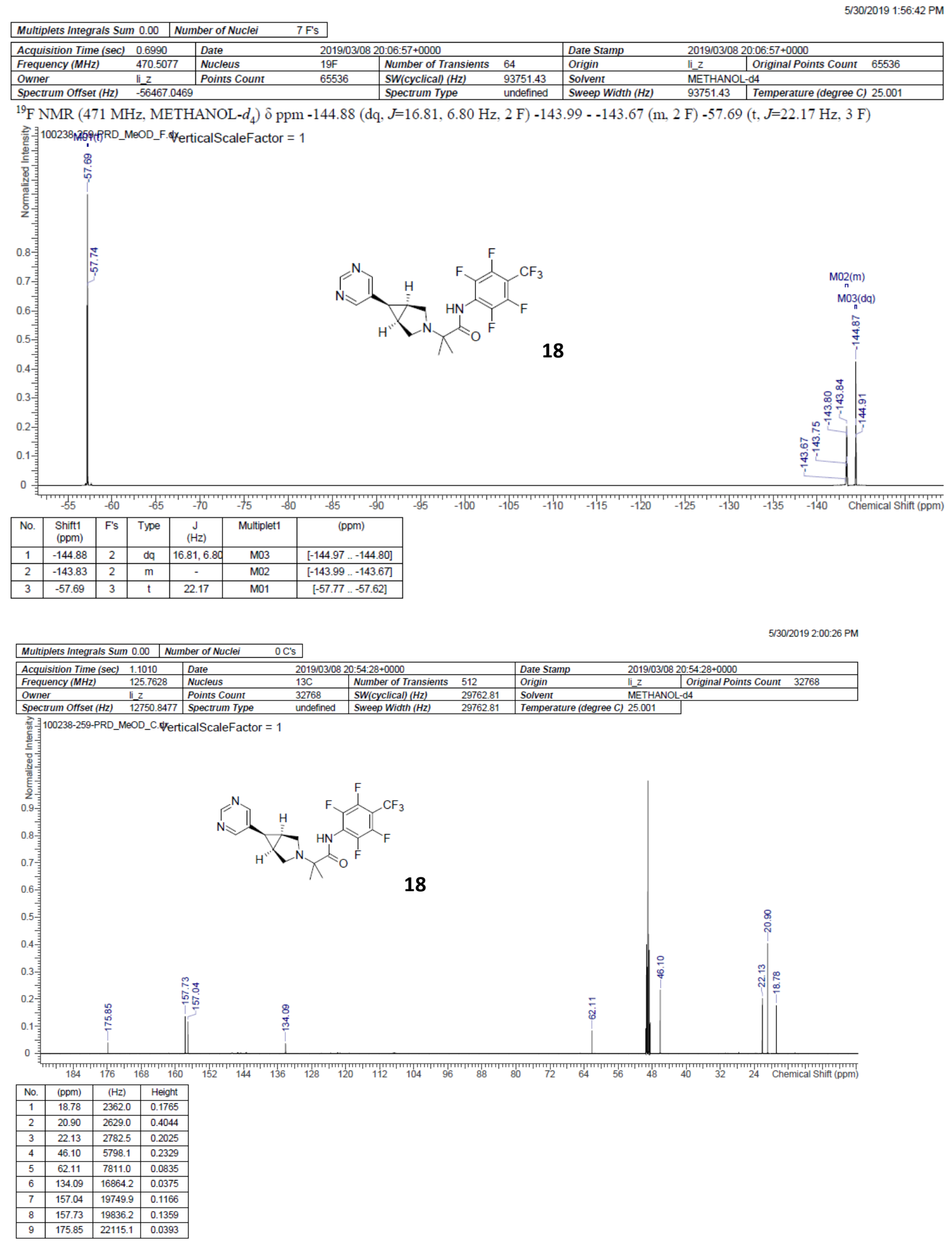
\begin{tabular}{|l|ll|}
\hline Multiplets Integrals Sum 19.31 & Number of Nuclei 19 H's \\
\hline
\end{tabular}

\begin{tabular}{|c|c|c|c|c|c|c|c|c|c|}
\hline Acquisition Time (sec) & 1.6383 & Date & \multicolumn{3}{|c|}{$2019 / 05 / 2321: 51: 06+0000$} & Date Stamp & \multicolumn{3}{|c|}{$2019 / 05 / 2321: 51: 06+0000$} \\
\hline Frequency $(\mathrm{MHz})$ & 500.1030 & Nucleus & $1 \mathrm{H}$ & Number of Transients & 8 & Origin & li_z & \begin{tabular}{|l} 
Original Points Count \\
\end{tabular} & 16384 \\
\hline Owner & lijz & Points Count & 16384 & SW(cyclical) $(\mathrm{Hz})$ & 10000.61 & \begin{tabular}{|l|} 
Solvent \\
\end{tabular} & \multicolumn{3}{|c|}{ CHLOROFORM-d } \\
\hline
\end{tabular}

\begin{tabular}{|ll|ll|ll|l|l|}
\hline Owner & lijz & Points Count & 16384 & SW(cyclical) $(\mathrm{Hz})$ & 10000.61 & Solvent & CHLOROFORM- \\
\hline Spectrum Offset $(\mathrm{Hz})$ & 2988.2705 & Spectrum Type & undefined & Sweep Width $(\mathrm{Hz})$ & 10000.61 & Temperature (degree C) 25.000 \\
\hline
\end{tabular}

${ }^{1} \mathrm{H}$ NMR $(500 \mathrm{MHz}$, CHLOROFORM- $d) \delta \mathrm{ppm} 1.07$ (s, $\left.6 \mathrm{H}\right) 2.06-2.12(\mathrm{~m}, 2 \mathrm{H}) 2.46(\mathrm{t}, J=7.94 \mathrm{~Hz}, 1 \mathrm{H}) 2.75-2.82(\mathrm{~m}, 2 \mathrm{H}) 2.92(\mathrm{~d}, J=9.77 \mathrm{~Hz}$, $2 \mathrm{H}) 3.91(\mathrm{~s}, 3 \mathrm{H}) 6.25(\mathrm{br} \mathrm{s}, 1 \mathrm{H}) 8.45(\mathrm{~d}, J=2.44 \mathrm{~Hz}, 1 \mathrm{H}) 8.63(\mathrm{~d}, J=2.44 \mathrm{~Hz}, 1 \mathrm{H})$

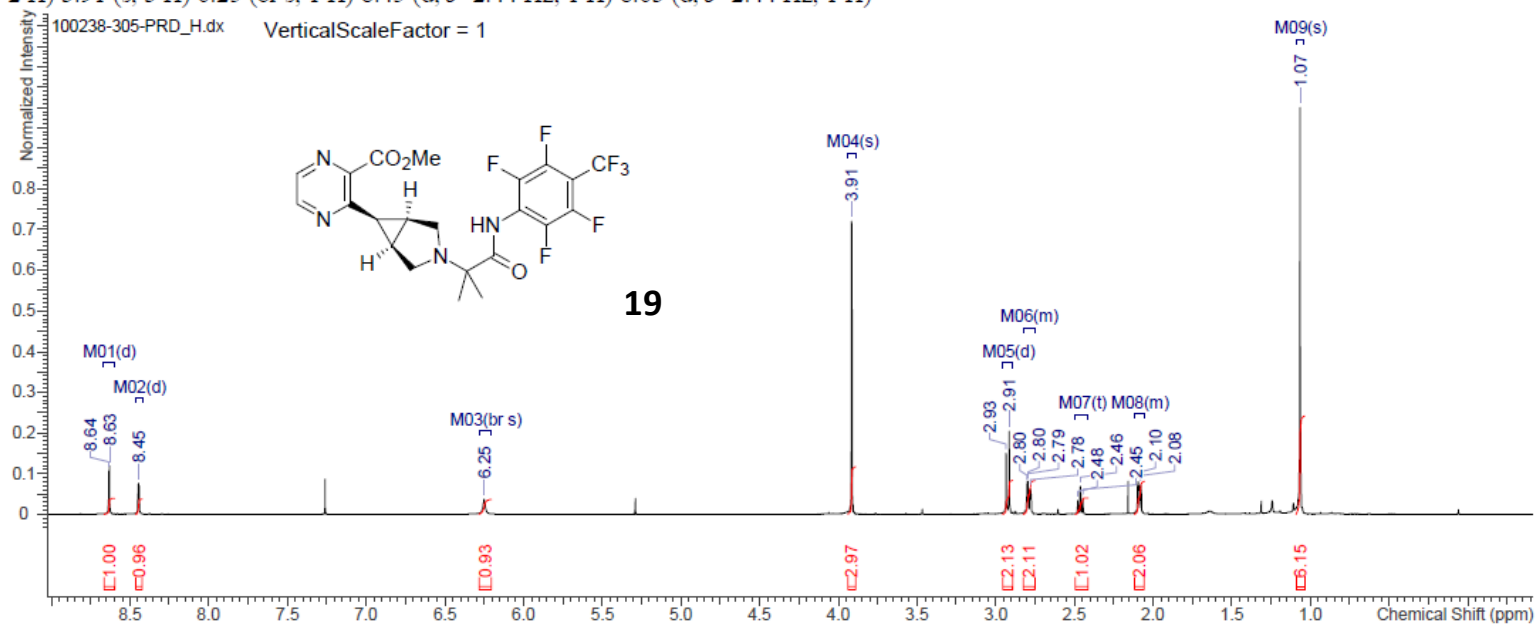

\begin{tabular}{|c|c|c|c|c|}
\hline No. & $(\mathrm{ppm})$ & Value & Absolute Value & Non-Negative Value \\
\hline 1 & {$[1.04 \ldots 1.09]$} & 6.146 & $1.139 \mathrm{e}+9$ & 6.146 \\
\hline 2 & {$[2.06 \ldots 2.12]$} & 2.057 & $3.811 \mathrm{e}+8$ & 2.057 \\
\hline 3 & {$[2.41 . .2 .49]$} & 1.018 & $1.886 \mathrm{e}+8$ & 1.018 \\
\hline 4 & {$[2.75 \ldots 2.82]$} & 2.106 & $3.901 \mathrm{e}+8$ & 2.106 \\
\hline 5 & {$[2.89 \ldots 2.95]$} & 2.131 & $3.948 \mathrm{e}+8$ & 2.131 \\
\hline 6 & {$[3.89 \ldots 3.94]$} & 2.969 & $5.500 \mathrm{e}+8$ & 2.969 \\
\hline 7 & {$[6.20 \ldots 6.28]$} & 0.931 & $1.724 \mathrm{e}+8$ & 0.931 \\
\hline 8 & {$[8.42 \ldots 8.46]$} & 0.955 & $1.770 \mathrm{e}+8$ & 0.955 \\
\hline 9 & {$[8.60 \ldots 8.67]$} & 1.000 & $1.852 \mathrm{e}+8$ & 1.000 \\
\hline
\end{tabular}

5/31/2019 9:44:05 AM

\begin{tabular}{|l|l|}
\hline Multiplets Integrals Sum 0.00 & Number of Nuclei \\
\hline
\end{tabular}

\begin{tabular}{|c|c|c|c|c|c|c|c|c|}
\hline Acquisition Time (sec) & 0.6990 & Date & \multicolumn{3}{|c|}{$2019 / 05 / 2321: 54: 22+0000$} & Date Stamp & \multicolumn{2}{|c|}{$2019 / 05 / 2321: 54: 22+0000$} \\
\hline Frequency $(\mathrm{MHz})$ & 470.5077 & Nucleus & $19 \mathrm{~F}$ & Number of Transients & 64 & Origin & li_z & Original Points Count 65536 \\
\hline Owner & lijz & Points Count & 65536 & SW(cyclical) $(\mathrm{Hz})$ & 93751.43 & Solvent & \multicolumn{2}{|c|}{ CHLOROFORM-d } \\
\hline Spectrum Offset $(\mathrm{Hz})$ & -56467.046 & & & Spectrum Type & undefined & Sweep Width $(\mathrm{Hz})$ & 93751.43 & Temperature (degree C) 25.001 \\
\hline
\end{tabular}

${ }^{19}$ F NMR (471 MHz, CHLOROFORM- $d$ ) $\delta$ ppm -143.39 - -143.22 (m, 2 F) -141.32 - -141.03 (m, 2 F) -56.06 (t, J=21.46 Hz, 3 F)

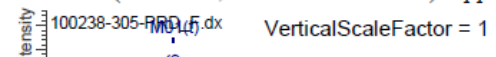

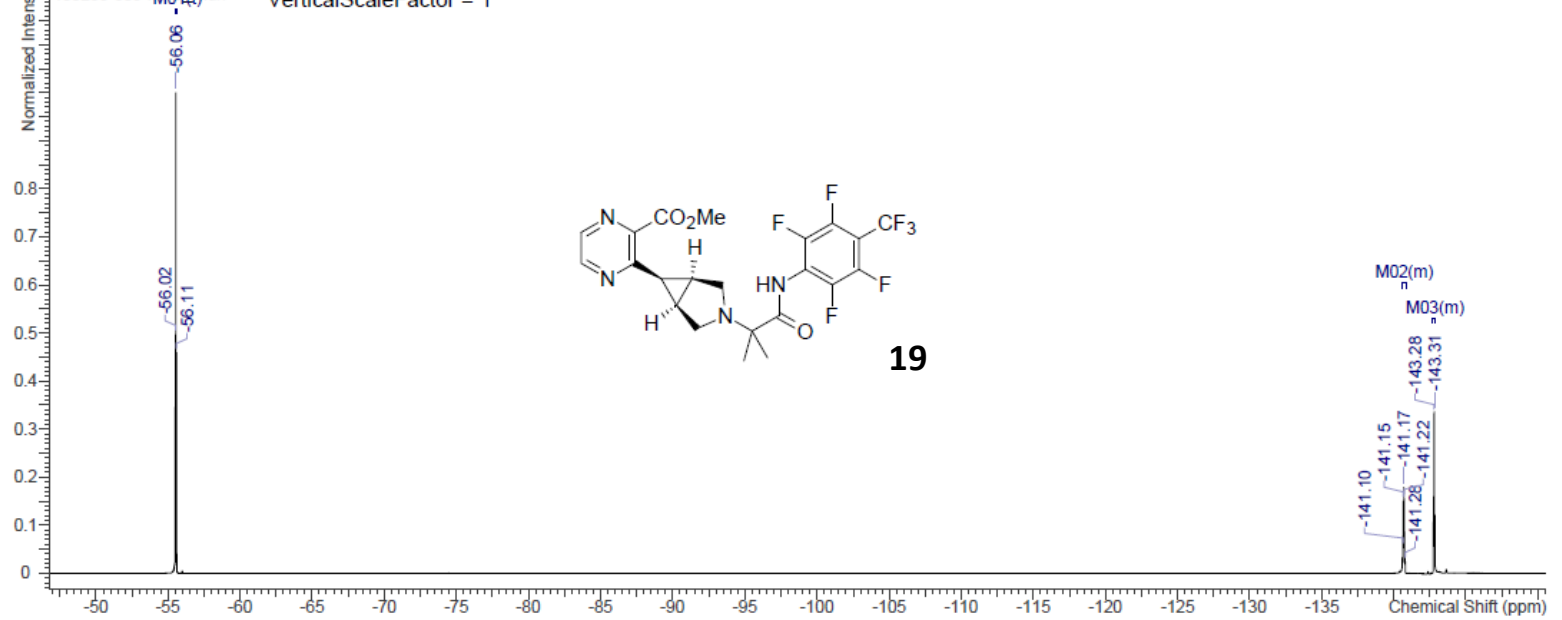

\begin{tabular}{|c|c|c|c|c|c|c|}
\hline No. & $\begin{array}{c}\text { Shift1 } \\
(\mathrm{ppm})\end{array}$ & F's & Type & $\begin{array}{c}\mathrm{J} \\
(\mathrm{Hz})\end{array}$ & Multiplet1 & $(\mathrm{ppm})$ \\
\hline 1 & -143.29 & 2 & $\mathrm{~m}$ & - & $\mathrm{M} 03$ & {$[-143.39 \ldots-143.22]$} \\
\hline 2 & -141.17 & 2 & $\mathrm{~m}$ & - & $\mathrm{M} 02$ & {$[-141.32 \ldots-141.03]$} \\
\hline 3 & -56.06 & 3 & $\mathrm{t}$ & 21.46 & M01 & {$[-56.17 \ldots-55.98]$} \\
\hline
\end{tabular}


\begin{tabular}{|l|ll|}
\hline Multiplets Integrals Sum 0.00 & Number of Nuclei 0 C's \\
\hline \hline
\end{tabular}

5/31/2019 9:49:21 AM

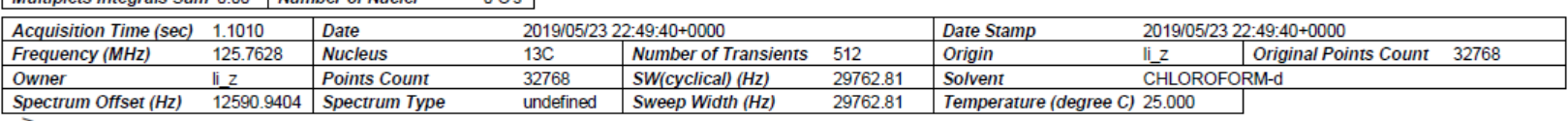

VerticalScaleFactor $=1$

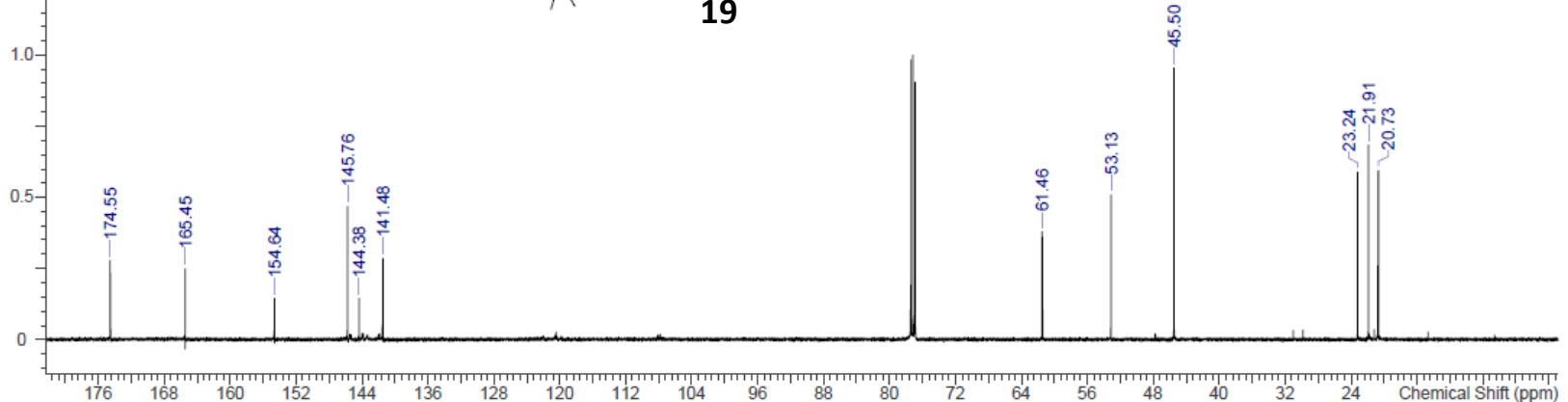

\begin{tabular}{|c|c|c|c|c|c|c|c|c|c|c|c|c|c|c|c|}
\hline No. & (ppm) & $(\mathrm{Hz})$ & Height & No. & (ppm) & $(\mathrm{Hz})$ & Height & No. & (ppm) & $(\mathrm{Hz})$ & Height & No. & (ppm) & $(\mathrm{Hz})$ & Height \\
\hline 1 & 20.73 & 2607.2 & 0.5943 & 4 & 45.50 & 5721.8 & 0.9547 & 7 & 141.48 & 17793.3 & 0.2847 & 10 & 154.64 & 19447.4 & 0.1451 \\
\hline 2 & 21.91 & 2755.2 & 0.6826 & 5 & 53.13 & 6681.9 & 0.5080 & 8 & 144.38 & 18157.6 & 0.1449 & 11 & 165.45 & 20807.1 & 0.2483 \\
\hline 3 & 23.24 & 2922.4 & 0.5876 & 6 & 61.46 & 7729.2 & 0.3784 & 9 & 145.76 & 18331.0 & 0.4668 & 12 & 174.55 & 21952.5 & 0.2767 \\
\hline
\end{tabular}

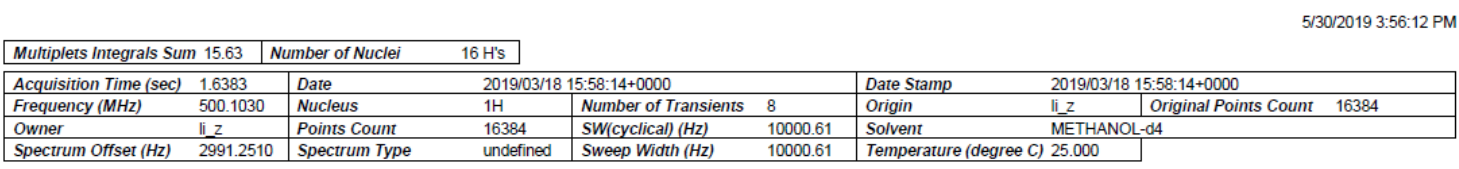

${ }^{1} \mathrm{H}$ NMR $\left(500 \mathrm{MHz}, \mathrm{METHANOL}-d_{4}\right) \delta \mathrm{ppm} 1.09(\mathrm{~s}, 6 \mathrm{H}) 2.02-2.06(\mathrm{~m}, 2 \mathrm{H}) 2.13(\mathrm{t}, J=7.94 \mathrm{~Hz}, 1 \mathrm{H}) 2.93-2.98$ (m, $\left.2 \mathrm{H}\right) 3.06$ (d, J=9.77 Hz, 2

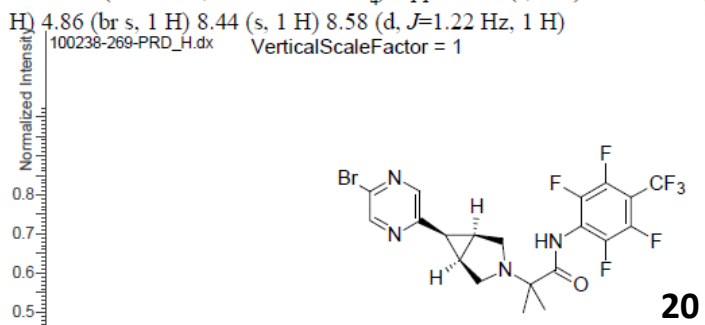

20

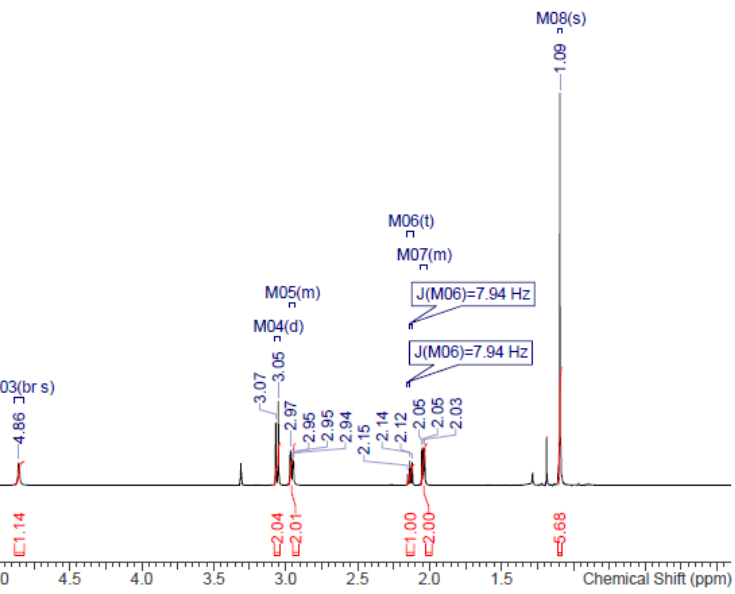

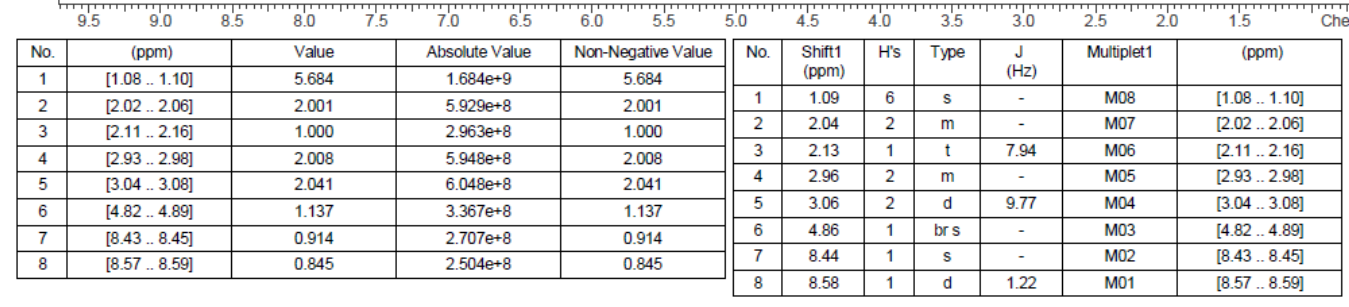


\begin{tabular}{|l|l|}
\hline Multiplets Integrals Sum 0.00 & Number of Nuclei $7 \mathrm{~F}$ 's \\
\hline
\end{tabular}

\begin{tabular}{|c|c|c|c|c|c|c|c|c|}
\hline Acquisition Time (sec) & 0.6990 & Date & \multicolumn{3}{|c|}{$2019 / 03 / 18$ 16:03:30+0000 } & Date Stamp & \multicolumn{2}{|c|}{$2019 / 03 / 18$ 16:03:30+0000 } \\
\hline Frequency $(\mathrm{MHz})$ & 470.5077 & Nucleus & $19 \mathrm{~F}$ & Number of Transients & 64 & \begin{tabular}{|l} 
Origin \\
\end{tabular} & li_z & Original Points Count 65536 \\
\hline Owner & lijz & Points Count & 65536 & SW(cyclical) $(\mathrm{Hz})$ & 93751.43 & Solvent & \multicolumn{2}{|c|}{ METHANOL-d4 } \\
\hline Spectrum Offset $(\mathrm{Hz})$ & -56467.046 & & & Spectrum Type & undefined & Sweep Width $(\mathrm{Hz})$ & 93751.43 & Temperature (degree C) 25.001 \\
\hline
\end{tabular}

\begin{tabular}{|ll|ll|ll|l}
\hline Spectrum Offset $(\mathrm{Hz})$ & -56467.0469 & Spectrum Type & undefined & Sweep Width $(\mathrm{Hz})$ & 93751.43 & Temperature (degree C) 25.001 \\
\hline
\end{tabular}

${ }^{19} \mathrm{~F}$ NMR $\left(471 \mathrm{MHz}, \mathrm{METHANOL}-d_{4}\right) \delta \mathrm{ppm}-144.72--144.55(\mathrm{~m}, 2 \mathrm{~F})-143.73--143.48(\mathrm{~m}, 2 \mathrm{~F})-57.63(\mathrm{t}, J=21.46 \mathrm{~Hz}, 3 \mathrm{~F})$

蛋表100238-269-PRD_F.dx VerticalScaleFactor $=1$

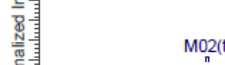

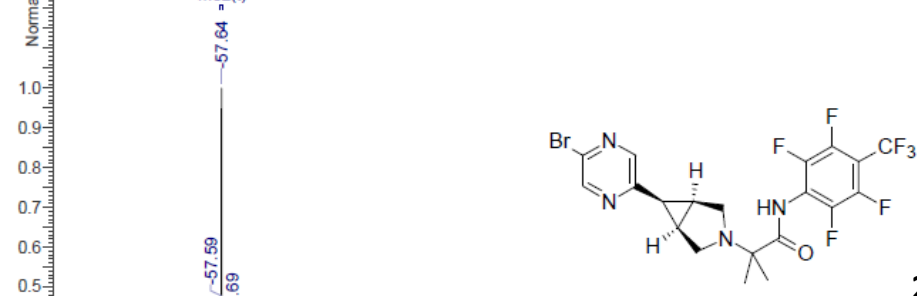

20

$\mathrm{MO3}_{\mathrm{n}}(\mathrm{m})$

MO4(m)

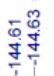
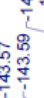

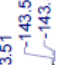

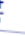

0

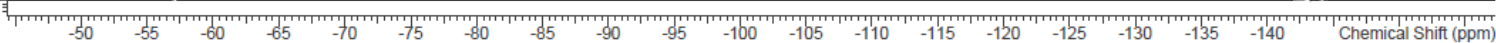

\begin{tabular}{|c|c|c|c|c|c|c|}
\hline No. & $\begin{array}{c}\text { Shift1 } \\
\text { (ppm) }\end{array}$ & F's & Type & $\begin{array}{c}\text { J } \\
(\mathrm{Hz})\end{array}$ & Multiplet1 & (ppm) \\
\hline 1 & -144.62 & 2 & $\mathrm{~m}$ & - & M04 & {$[-144.72 \ldots-144.55]$} \\
\hline 2 & -143.60 & 2 & $\mathrm{~m}$ & - & M03 & {$[-143.73 \ldots-143.48]$} \\
\hline 3 & -57.63 & 3 & $\mathrm{t}$ & 21.46 & M02 & {$[-57.71 \ldots-57.57]$} \\
\hline
\end{tabular}

5/30/2019 4:07:52 PM

\begin{tabular}{|l|l|}
\hline Multiplets Integrals Sum 0.00 & Number of Nuclei \\
\hline
\end{tabular}

\begin{tabular}{|c|c|c|c|c|c|c|c|c|c|}
\hline Acquisition Time (sec) & 1.1010 & Date & \multicolumn{3}{|c|}{ 2019/03/18 16:24:53+0000 } & Date Stamp & \multicolumn{3}{|c|}{ 2019/03/18 16:24:53+0000 } \\
\hline Frequency $(\mathrm{MHz})$ & 125.7628 & Nucleus & $13 \mathrm{C}$ & Number of Transients & 512 & Origin & li_z & \begin{tabular}{|l|} 
Original Points Coumt \\
\end{tabular} & 32768 \\
\hline Owner & li_z & Points Count & 32768 & SW(cyclical) $(\mathrm{Hz})$ & 29762.81 & Solvent & & & \\
\hline
\end{tabular}

\begin{tabular}{|ll|ll|ll|l|l} 
OWner & li_z & Points Count & 32768 & SW(cyclical) $(\mathrm{Hz})$ & 29762.81 & Solvent & METHANOL-04 \\
\hline Spectrum Offset $(\mathrm{Hz})$ & 12749.9424 & Spectrum Type & undefined & Sweep Width $(\mathrm{Hz})$ & 29762.81 & Temperature (degree C) 25.001
\end{tabular}

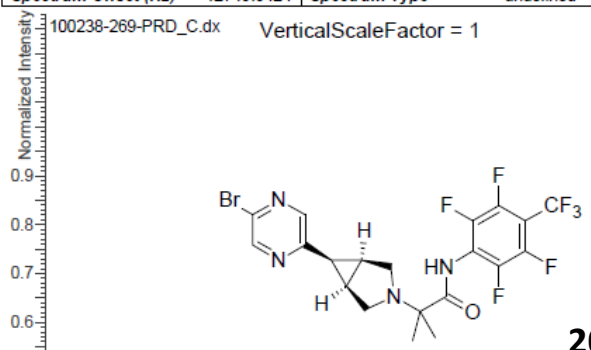

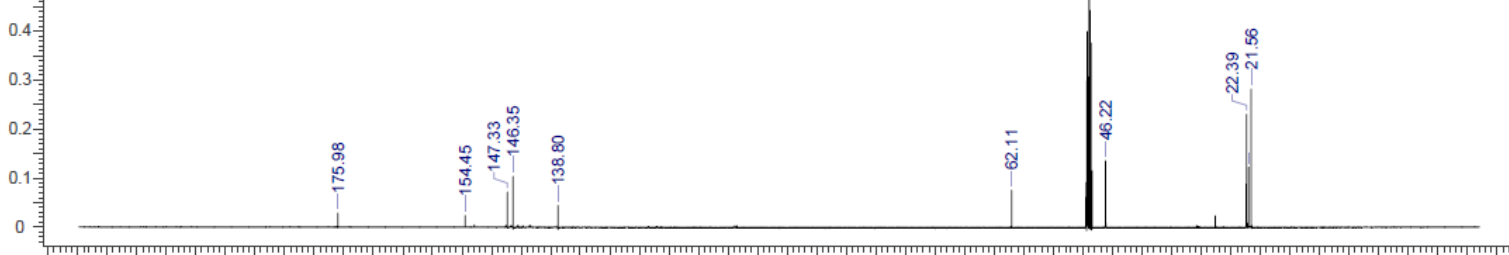

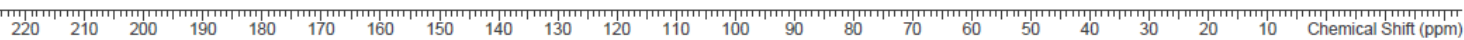

\begin{tabular}{|c|c|c|c|}
\hline No. & $(\mathrm{ppm})$ & $(\mathrm{Hz})$ & Height \\
\hline 1 & 21.56 & 2711.7 & 0.2820 \\
\hline 2 & 21.99 & 2765.3 & 0.1218 \\
\hline 3 & 22.39 & 2815.2 & 0.2302 \\
\hline 4 & 46.22 & 5812.7 & 0.1353 \\
\hline 5 & 62.11 & 7811.0 & 0.0754 \\
\hline 6 & 138.80 & 17455.5 & 0.0436 \\
\hline 7 & 146.35 & 18405.6 & 0.1030 \\
\hline 8 & 147.33 & 18528.2 & 0.0714 \\
\hline 9 & 154.45 & 19423.8 & 0.0229 \\
\hline 10 & 175.98 & 22131.5 & 0.0274 \\
\hline
\end{tabular}


5/31/2019 10:00:43 AM

\begin{tabular}{|c|c|c|c|c|c|c|c|c|c|}
\hline Multiplets Integrals Sum & $n 19.02$ & Number of Nuclei & $18 \mathrm{H}$ 's & & & & & & \\
\hline Acquisition Time (sec) & 1.6383 & Date & $2019 / 03 / 21$ & $2: 00: 31+0000$ & & Date Stamp & 2019 & $22: 00: 31+0000$ & \\
\hline Frequency $(\mathrm{MHz})$ & 500.1030 & Nucleus & $1 \mathrm{H}$ & Number of Transients & 8 & \begin{tabular}{|l|} 
Origin \\
\end{tabular} & li_z & Original Points Count & 16384 \\
\hline Owner & liz & Points Count & 16384 & SW(cyclical) $(\mathrm{Hz})$ & 10000.61 & \begin{tabular}{|l|} 
Solvent \\
\end{tabular} & $\mathrm{CHL}$ & RM-d & \\
\hline
\end{tabular}

${ }^{1} \mathrm{H}$ NMR $(500 \mathrm{MHz}$, CHLOROFORM- $d$ ) $\delta \mathrm{ppm} 1.08(\mathrm{~s}, 6 \mathrm{H}) 1.97-2.06(\mathrm{~m}, 2 \mathrm{H}) 2.27$ (t, $J=7.94 \mathrm{~Hz}, 1 \mathrm{H}) 2.87-2.97(\mathrm{~m}, 2 \mathrm{H}) 3.05(\mathrm{~d}, J=9.16 \mathrm{~Hz}$, $2 \mathrm{H}) 7.77(\mathrm{dd}, J=8.55,1.83 \mathrm{~Hz}, 1 \mathrm{H}) 7.92(\mathrm{~d}, J=8.55 \mathrm{~Hz}, 1 \mathrm{H}) 7.96(\mathrm{~s}, 1 \mathrm{H}) 8.52(\mathrm{~d}, J=1.83 \mathrm{~Hz}, 1 \mathrm{H}) 8.63(\mathrm{~d}, J=1.83 \mathrm{~Hz}, 1 \mathrm{H})$

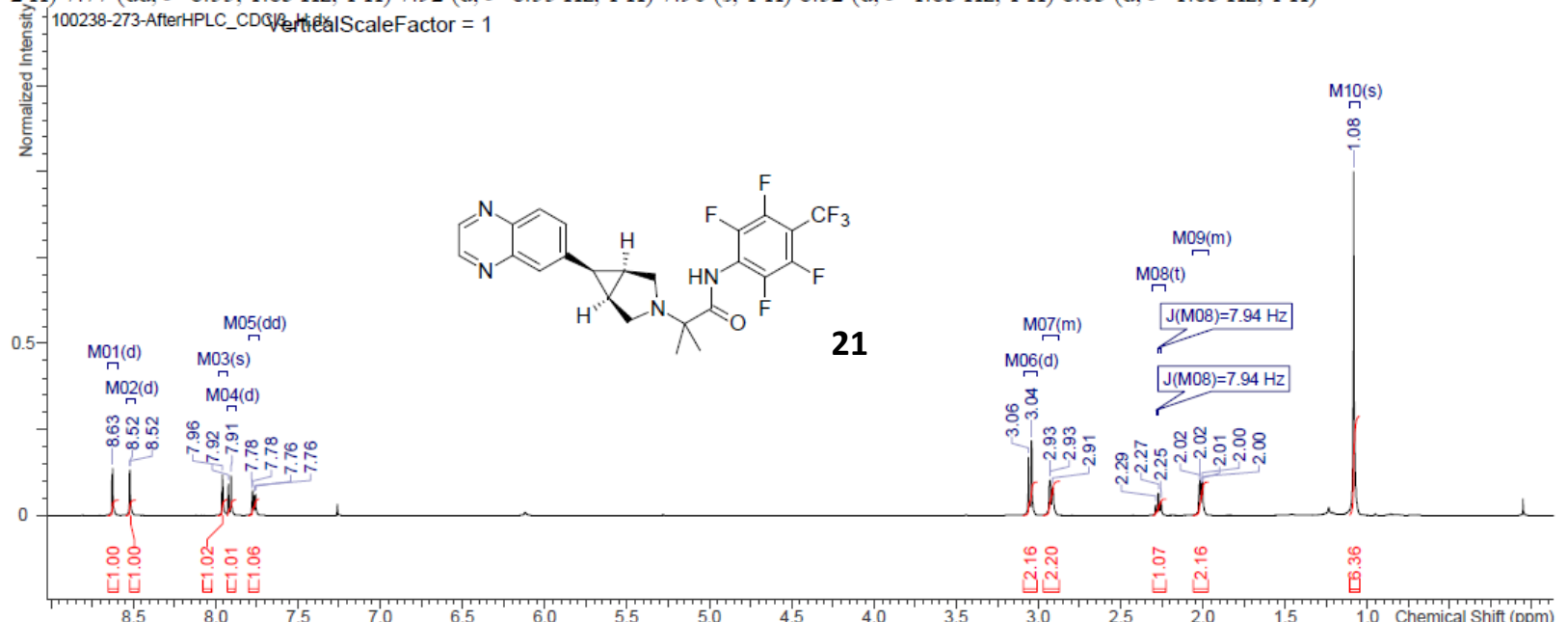

\begin{tabular}{|c|c|c|c|c|c|c|c|c|c|c|c|c|c|}
\hline No. & $\begin{array}{l}\text { Shift1 } \\
(\mathrm{ppm})\end{array}$ & H's & Type & $\begin{array}{c}\mathrm{J} \\
(\mathrm{Hz})\end{array}$ & Multiplet1 & (ppm) & No. & $\begin{array}{l}\text { Shift1 } \\
\text { (ppm) }\end{array}$ & H's & Type & $\begin{array}{c}\mathrm{J} \\
(\mathrm{Hz})\end{array}$ & Multiplet1 & (ppm) \\
\hline 1 & 1.08 & 6 & $s$ & - & M10 & [1.05 . . 1.11] & 6 & 7.77 & 1 & dd & $8.55,1.83$ & M05 & {$[7.74 \ldots 7.80]$} \\
\hline 2 & 2.01 & 2 & $\mathrm{~m}$ & - & M09 & {$\left[\begin{array}{llll}1.97 & .2 .06]\end{array}\right.$} & 7 & 7.92 & 1 & d & 8.55 & M04 & {$[7.88 \ldots 7.93]$} \\
\hline 3 & 2.27 & 1 & $t$ & 7.94 & M08 & {$[2.23 \ldots 2.30]$} & 8 & 7.96 & 1 & $\mathrm{~s}$ & - & M03 & {$[7.93 \ldots 7.99]$} \\
\hline 4 & 2.92 & 2 & $\mathrm{~m}$ & - & M07 & {$\left[\begin{array}{llll}2.87 & \ldots & 2.97\end{array}\right]$} & 9 & 8.52 & 1 & $d$ & 1.83 & M02 & [8.49 .. 8.55] \\
\hline 5 & 3.05 & 2 & d & 9.16 & M06 & [3.01 . 3.09] & 10 & 8.63 & 1 & $d$ & 1.83 & M01 & {$[8.59 \ldots .8 .65]$} \\
\hline
\end{tabular}

5/31/2019 10:06:46 AM

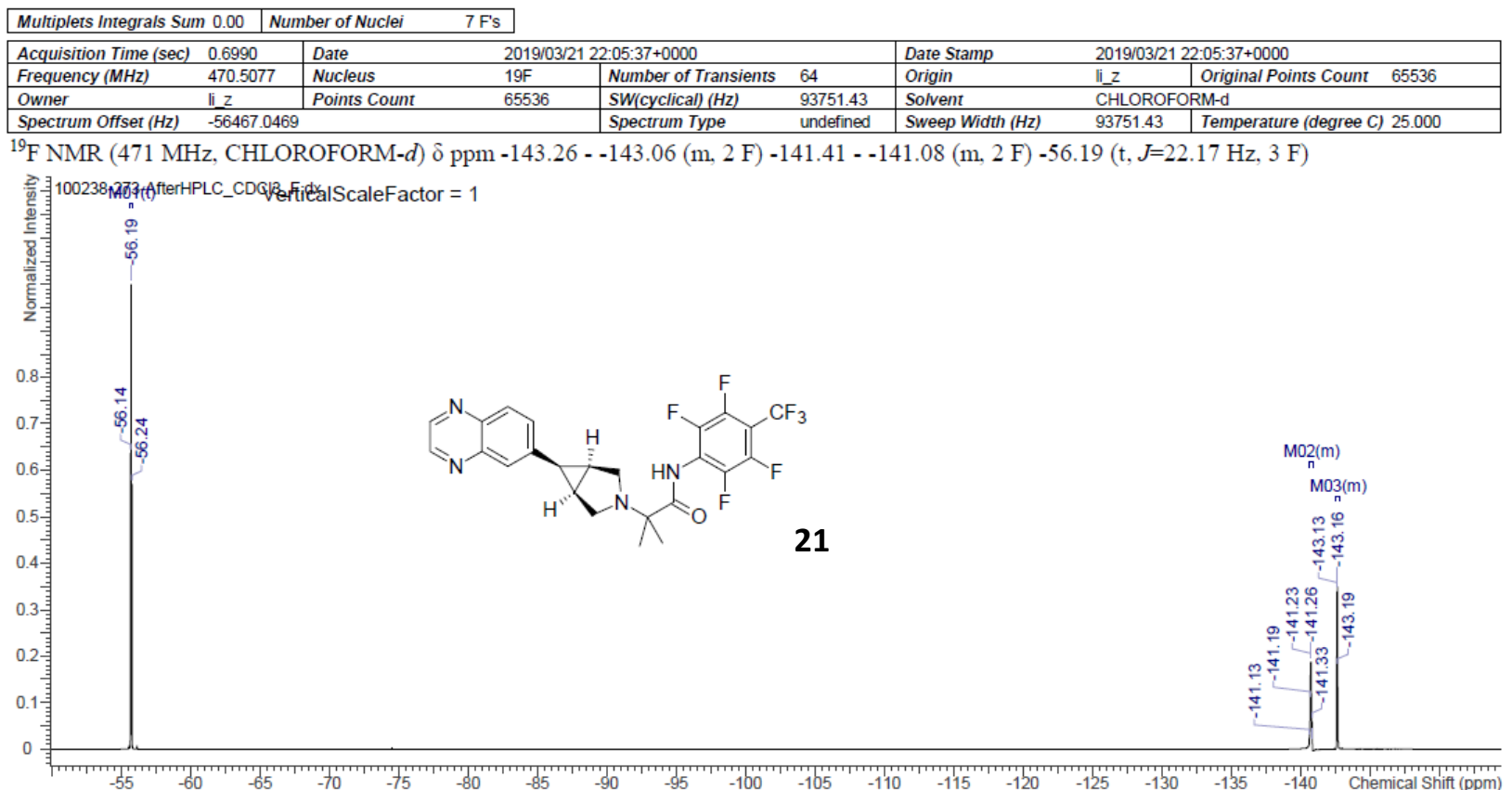

\begin{tabular}{|c|c|c|c|c|c|c|}
\hline No. & $\begin{array}{c}\text { Shift1 } \\
(\mathrm{ppm})\end{array}$ & F's & Type & $\begin{array}{c}\text { J } \\
(\mathrm{Hz})\end{array}$ & Multiplet1 & $(\mathrm{ppm})$ \\
\hline 1 & -143.15 & 2 & $\mathrm{~m}$ & - & $\mathrm{M} 03$ & {$[-143.26 \ldots-143.06]$} \\
\hline 2 & -141.24 & 2 & $\mathrm{~m}$ & - & $\mathrm{M} 02$ & {$[-141.41 \ldots-141.08]$} \\
\hline 3 & -56.19 & 3 & $\mathrm{t}$ & 22.17 & M01 & {$[-56.30 \ldots-56.09]$} \\
\hline
\end{tabular}




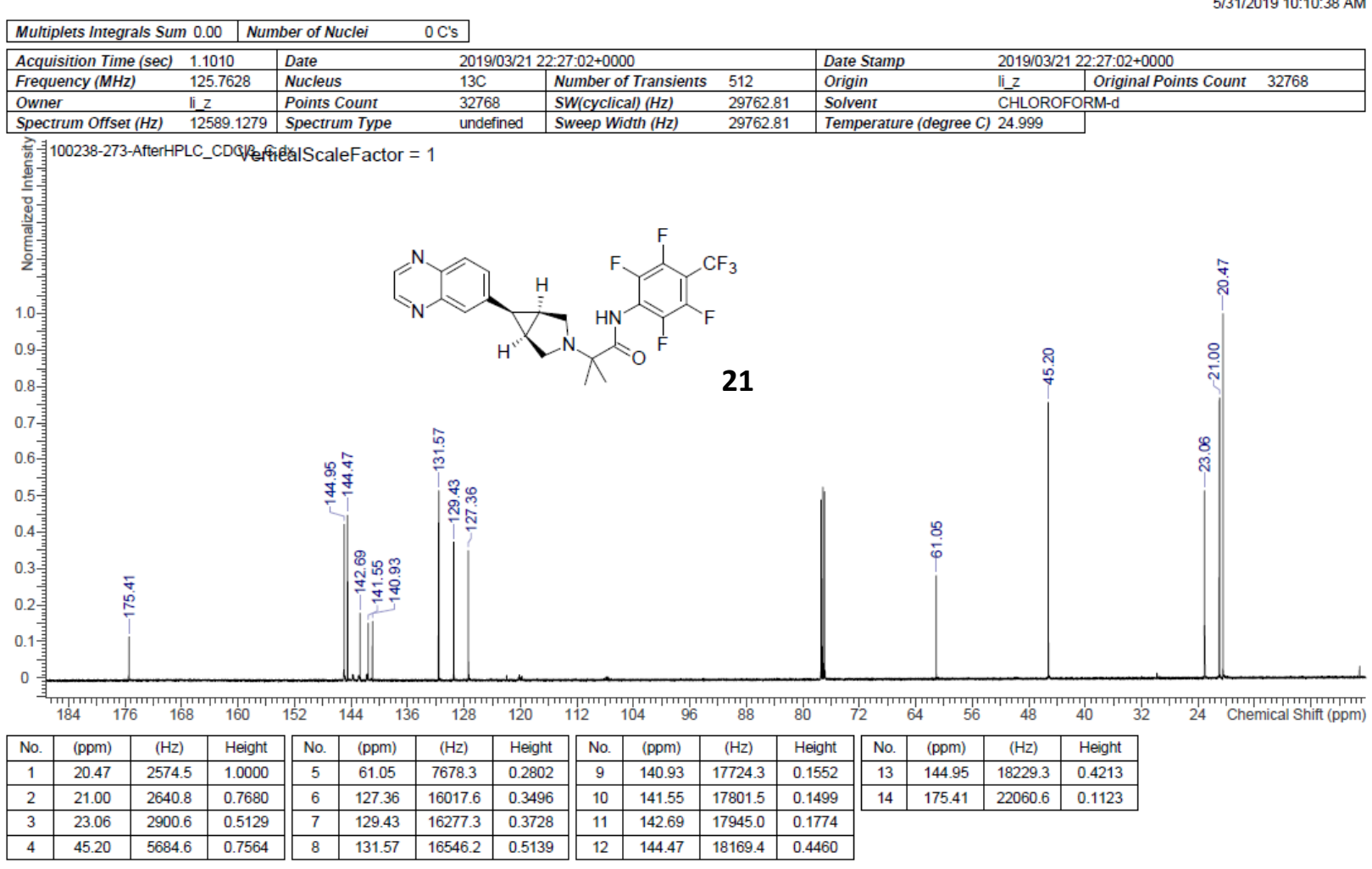

$6 / 22 U 19$ 4:28:24 PM \begin{tabular}{|l|l|}
\hline Multiplets Integrals Sum 23.47 & Number of Nuclei $\quad 23 \mathrm{H}$ 's \\
\hline
\end{tabular}

\begin{tabular}{|ll|ll|ll|l|l|}
\hline Acquisition Time $(\mathrm{sec})$ & 1.6383 & Date & $2019 / 05 / 22$ & $17: 29: 16+0000$ & Date Stamp & $2019 / 05 / 2217: 29: 16+0000$ \\
\hline Frequency $(\mathrm{MHz})$ & 500.1030 & Nucleus & $1 \mathrm{H}$ & Number of Transients & 8 & Origin & li_z \\
\hline Owner & li_z & Points Count & 16384 & SW(cyclical) $(\mathrm{Hz})$ & 10000.61 & Solvent & CHLOROFORM-d \\
\hline Spectrum Offset $(\mathrm{Hz})$ & 2988.8752 & Spectrum Type & undefined & Sweep Width $(\mathrm{Hz})$ & 10000.61 & Temperature (degree C) 25.000 \\
\hline
\end{tabular}

${ }^{1} \mathrm{H}$ NMR $(500 \mathrm{MHz}$, CHLOROFORM-d) $\delta \mathrm{ppm} 1.08(\mathrm{~s}, 6 \mathrm{H}) 1.89-1.99(\mathrm{~m}, 2 \mathrm{H}) 2.05$ (t, J=7.33 Hz, $1 \mathrm{H}) 2.87-3.00$ (m, 4 H) 6.25 (br s, $\left.1 \mathrm{H}\right) 6.31$ $(\mathrm{d}, J=3.66 \mathrm{~Hz}, 1 \mathrm{H}) 7.38-7.46(\mathrm{~m}, 2 \mathrm{H}) 7.48(\mathrm{~d}, J=3.66 \mathrm{~Hz}, 1 \mathrm{H}) 7.53(\mathrm{t}, J=7.33 \mathrm{~Hz}, 1 \mathrm{H}) 7.77(\mathrm{~s}, 1 \mathrm{H}) 8.05-8.11(\mathrm{~m}, 2 \mathrm{H}) 8.43(\mathrm{~d}, J=1.83 \mathrm{~Hz}, 1$

$\mathrm{H})$

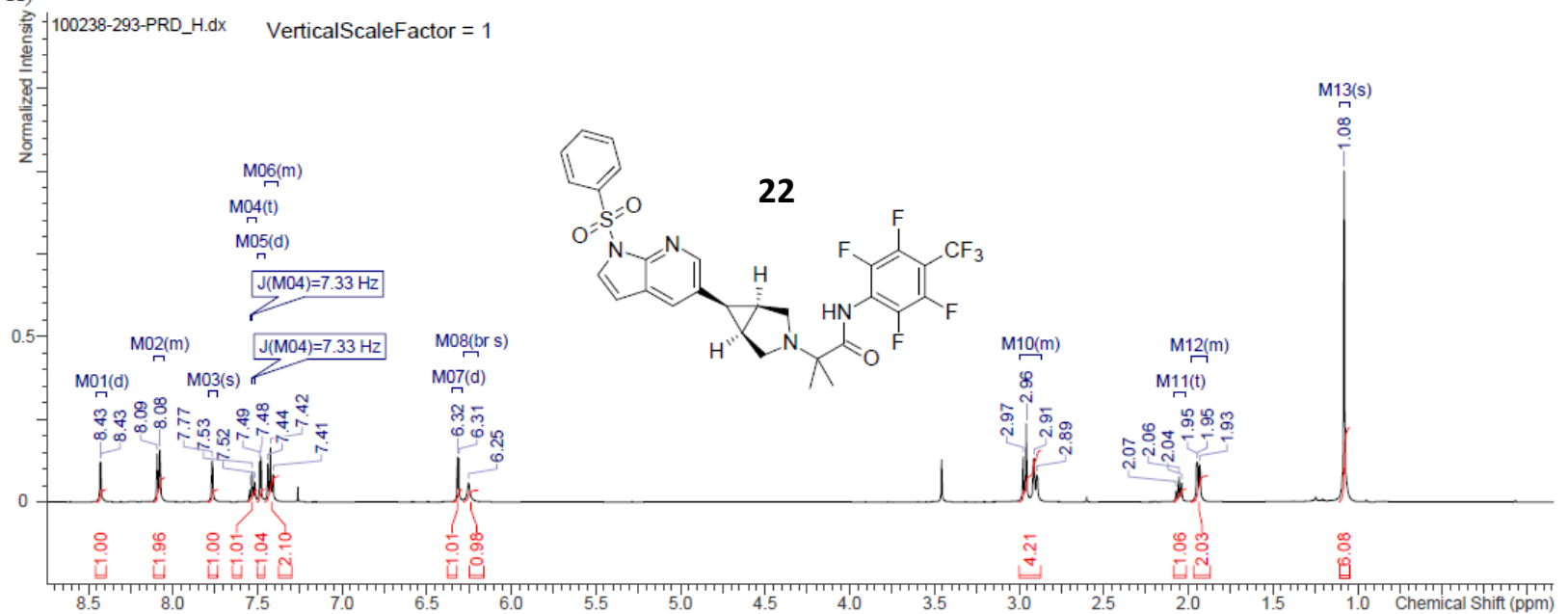

\begin{tabular}{|c|c|c|c|c|c|c|c|c|c|c|c|c|c|}
\hline No. & $\begin{array}{l}\text { Shift1 } \\
\text { (ppm) }\end{array}$ & H's & Type & $\begin{array}{c}\mathrm{J} \\
(\mathrm{Hz})\end{array}$ & Multiplet1 & (ppm) & No. & $\begin{array}{l}\text { Shift1 } \\
\text { (ppm) }\end{array}$ & H's & Type & $\begin{array}{c}\mathrm{J} \\
(\mathrm{Hz})\end{array}$ & Multiplet1 & (ppm) \\
\hline 1 & 1.08 & 6 & $\mathrm{~s}$ & - & M13 & {$[1.05 \ldots 1.11]$} & 7 & 7.42 & 2 & $\mathrm{~m}$ & - & M06 & {$[7.38 \ldots 7.46]$} \\
\hline 2 & 1.94 & 2 & $\mathrm{~m}$ & - & M12 & {$\left[\begin{array}{lll}1.89 & \ldots & 1.99\end{array}\right]$} & 8 & 7.48 & 1 & $d$ & 3.66 & M05 & {$[7.46 \ldots 7.50]$} \\
\hline 3 & 2.05 & 1 & $t$ & 7.33 & M11 & {$[2.01 \ldots 2.09]$} & 9 & 7.53 & 1 & $t$ & 7.33 & M04 & [7.50 ..7.56] \\
\hline 4 & 2.94 & 4 & $\mathrm{~m}$ & - & M10 & {$[2.87 \ldots 3.00]$} & 10 & 7.77 & 1 & $\mathrm{~s}$ & - & M03 & {$[7.73 \ldots 7.79]$} \\
\hline 5 & 6.25 & 1 & $\mathrm{brs}$ & - & M08 & {$\left[\begin{array}{llll}6.20 & \ldots & 6.28\end{array}\right]$} & 11 & 8.08 & 2 & $\mathrm{~m}$ & - & M02 & [8.05 .. 8.11] \\
\hline 6 & 6.31 & 1 & d & 3.66 & M07 & {$\left[\begin{array}{l}6.29 \\
. .6 .35]\end{array}\right.$} & 12 & 8.43 & 1 & $d$ & 1.83 & M01 & {$\left[\begin{array}{l}8.40 \\
.8 .46]\end{array}\right.$} \\
\hline
\end{tabular}


\begin{tabular}{|l|l|}
\hline Multiplets Integrals Sum 0.00 & Number of Nuclei 7 F's \\
\hline
\end{tabular}

\begin{tabular}{|c|c|c|c|c|c|c|c|c|c|}
\hline Acquisition Time (sec) & 0.6990 & Date & \multicolumn{3}{|c|}{$2019 / 05 / 2217: 34: 45+0000$} & Date Stamp & \multicolumn{3}{|c|}{$2019 / 05 / 22$ 17:34:45+0000 } \\
\hline Frequency $(\mathrm{MHz})$ & 470.5077 & Nucleus & $19 \mathrm{~F}$ & Number of Transients & 64 & Origin & li_z & Original Points Count & 65536 \\
\hline Owner & li_z & Points Count & 65536 & SW(cyclical) $(\mathrm{Hz})$ & 93751.43 & Solvent & \multicolumn{3}{|c|}{ CHLOROFORM-d } \\
\hline Spectrum Offset $(\mathrm{Hz})$ & $-56467.046 \mathrm{~S}$ & & & Spectrum Type & undefined & Sweep Width $(\mathrm{Hz})$ & 93751.43 & Temperature (degree $\mathrm{C}$ & 24.999 \\
\hline
\end{tabular}

${ }^{19}$ F NMR (471 MHz, CHLOROFORM- $d$ ) $\delta$ ppm -143.39 - -143.18 (m. 2 F) -141.32 - -141.00 (m. 2 F) -56.19 (t, J=22.17 Hz, 3 F)

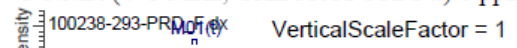

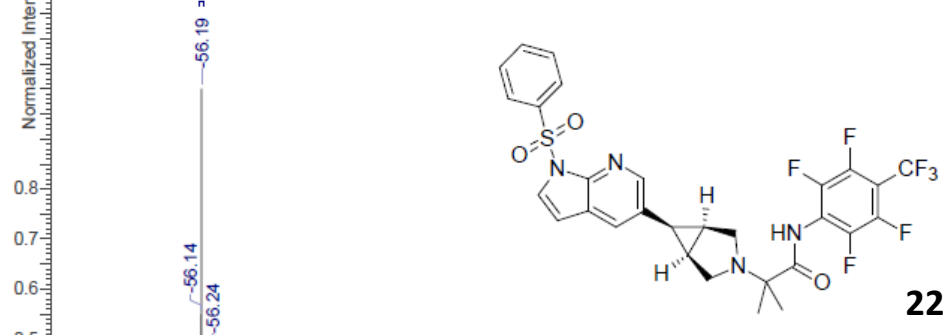

22

$\mathrm{MO2}(\mathrm{m})$

$\mathrm{MO3}_{\mathrm{n}}(\mathrm{m})$

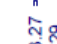

年

둔

$\frac{7}{4}$

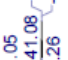

过

0

\begin{tabular}{|c|c|c|c|c|c|c|}
\hline No. & $\begin{array}{c}\text { Shift1 } \\
(\mathrm{ppm})\end{array}$ & $\mathrm{F}$ 's & Type & $\begin{array}{c}\mathrm{J} \\
(\mathrm{Hz})\end{array}$ & Multiplet1 & $(\mathrm{ppm})$ \\
\hline 1 & -143.28 & 2 & $\mathrm{~m}$ & - & $\mathrm{M} 03$ & {$[-143.39 \ldots-143.18]$} \\
\hline 2 & -141.16 & 2 & $\mathrm{~m}$ & - & $\mathrm{M} 02$ & {$[-141.32 \ldots-141.00]$} \\
\hline 3 & -56.19 & 3 & $\mathrm{t}$ & 22.17 & $\mathrm{M} 01$ & {$[-56.31 \ldots-56.07]$} \\
\hline
\end{tabular}

\begin{tabular}{|l|ll|}
\hline Multiplets Imtegrals Sum 0.00 & Number of Nuclei & 0 C's \\
\hline
\end{tabular}

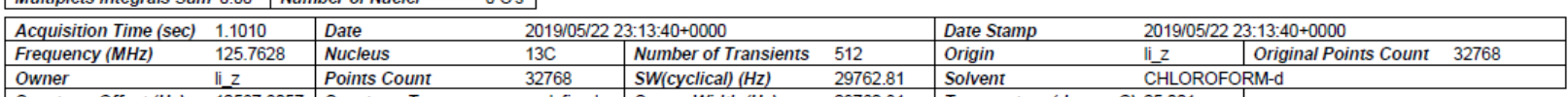

\begin{tabular}{|ll|ll|ll|l|l|}
\hline Owner & li_z & Points Count & 32768 & SW (cyclical) $(\mathrm{Hz})$ & 29762.81 & Solvent & CHLOROFORM-1 \\
\hline Spectrum Offset $(\mathrm{Hz})$ & 12587.3057 & Spectrum Type & undefined & Sweep Width $(\mathrm{Hz})$ & 29762.81 & Temperature (degree C) 25.001 \\
\hline
\end{tabular}

Spectrum Offset $(\mathrm{Hz}) \quad 12587.3057$ Spectrum Type

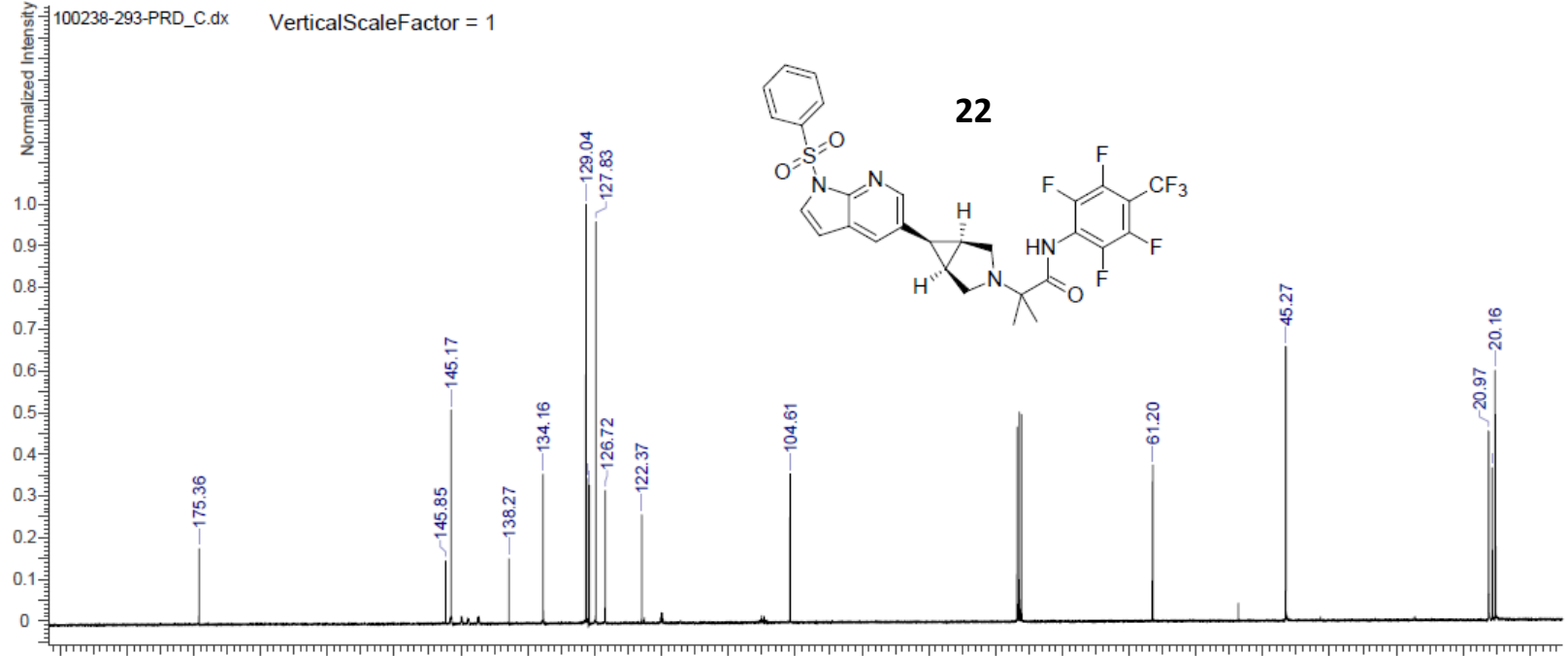

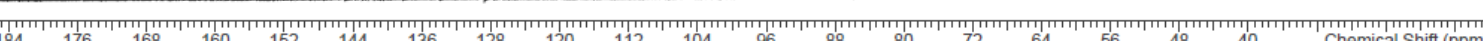

\begin{tabular}{|c|c|c|c|c|c|c|c|c|c|c|c|c|c|c|c|}
\hline No. & $(\mathrm{ppm})$ & $(\mathrm{Hz})$ & Height & No. & (ppm) & $(\mathrm{Hz})$ & Height & No. & $(\mathrm{ppm})$ & $(\mathrm{Hz})$ & Height & No. & $(\mathrm{ppm})$ & $(\mathrm{Hz})$ & Height \\
\hline 1 & 20.16 & 2535.4 & 0.6006 & 6 & 104.61 & 13155.5 & 0.3526 & 11 & 128.76 & 16192.9 & 0.3413 & 16 & 145.85 & 18342.9 & 0.1444 \\
\hline 2 & 20.54 & 2582.7 & 0.3682 & 7 & 122.37 & 15389.0 & 0.2538 & 12 & 129.04 & 16228.3 & 1.0000 & 17 & 175.36 & 22053.3 & 0.1729 \\
\hline 3 & 20.97 & 2637.2 & 0.4561 & 8 & 126.72 & 15936.7 & 0.3126 & 13 & 134.16 & 16872.3 & 0.3511 & & & & \\
\hline 4 & 45.27 & 5692.7 & 0.6588 & 9 & 127.83 & 16076.6 & 0.9576 & 14 & 138.27 & 17389.1 & 0.1491 & & & & \\
\hline & 61.20 & 7696.5 & 0.3736 & 10 & 128.64 & 16178.3 & 0.3249 & 15 & 145.17 & 18257.5 & 0.5061 & & & & \\
\hline
\end{tabular}




\begin{tabular}{|c|c|c|c|c|c|c|c|c|c|}
\hline Multiplets Integrals Sum & 20.83 & imber of Nuclei & $0 \mathrm{H}$ 's & & & & & & \\
\hline Acquisition Time (sec) & 1.6383 & Date & $2019 / 05 / 24$ & $8: 34: 00+0000$ & & Date Stamp & $2019 / 05$ & $8: 34: 00+0000$ & \\
\hline Frequency $(\mathrm{MHz})$ & 500.1030 & Nucleus & $1 \mathrm{H}$ & Number of Transients & 8 & Origin & liizz & Original Points Count & 16384 \\
\hline Owner & lijz & Points Count & 16384 & SW(cyclical) $(\mathrm{Hz})$ & 10000.61 & Solvent & $\mathrm{CHLOR}$ & RM-d & \\
\hline Spectrum Offset $(\mathrm{Hz})$ & 2988.2749 & Spectrum Type & undefined & Sweep Width $(\mathrm{Hz})$ & 10000.61 & Temperature (degree $C$ ) & 25.000 & & \\
\hline
\end{tabular}

${ }^{1} \mathrm{H}$ NMR $(500 \mathrm{MHz}$, CHLOROFORM- $d$ ) $\delta$ ppm 1.11 (s, $6 \mathrm{H}) 2.05-2.11(\mathrm{~m}, 3 \mathrm{H}) 2.91$ (br d, $\left.J=9.16 \mathrm{~Hz}, 2 \mathrm{H}\right) 3.11$ (d, J=9.16 Hz, $\left.2 \mathrm{H}\right) 3.98$ (s, $\left.3 \mathrm{H}\right)$ 6.67 (br s, $1 \mathrm{H}) 6.88(\mathrm{dd}, J=7.94,4.27 \mathrm{~Hz}, 1 \mathrm{H}) 8.04(\mathrm{dd}, J=7.94,1.22 \mathrm{~Hz}, 1 \mathrm{H}) 8.08(\mathrm{dd}, J=4.27,1.83 \mathrm{~Hz}, 1 \mathrm{H})$
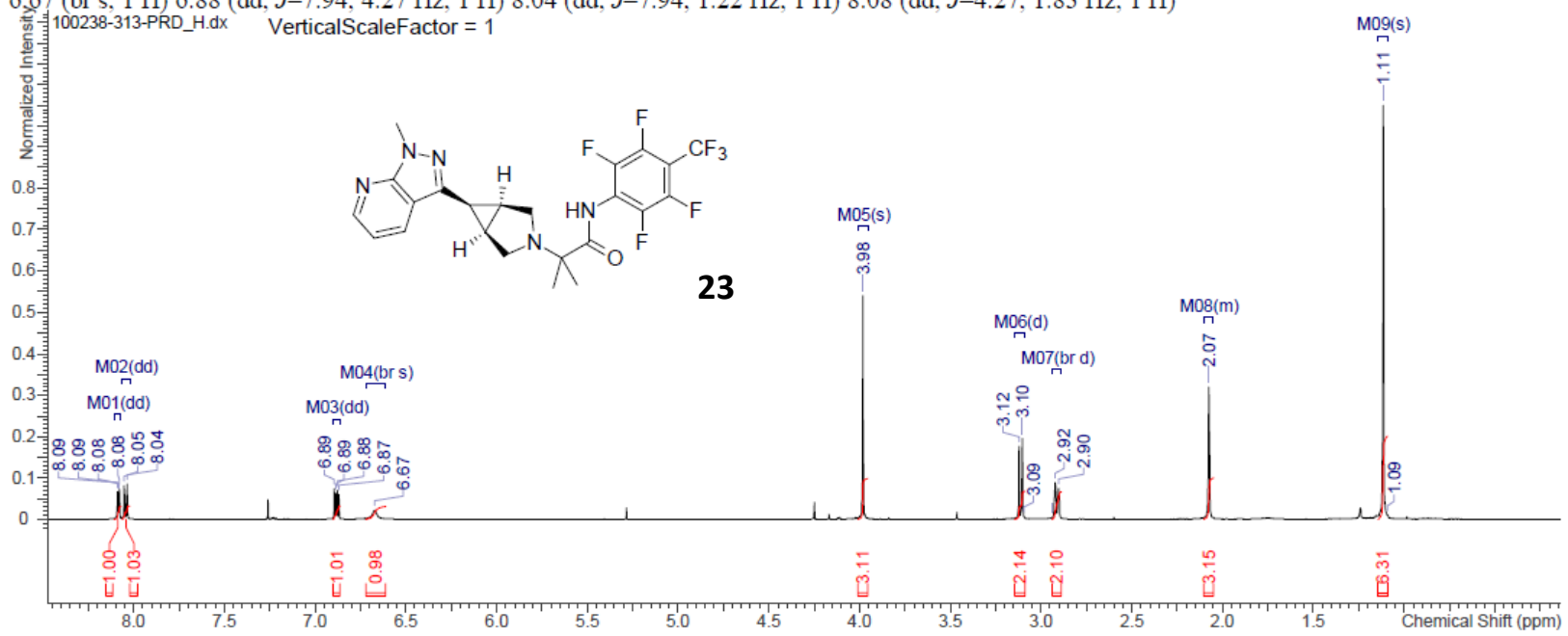

\begin{tabular}{|c|c|c|c|c|}
\hline No. & $(\mathrm{ppm})$ & Value & Absolute Value & Non-Negative Value \\
\hline 1 & {$[1.09 \ldots 1.14]$} & 6.313 & $2.403 \mathrm{e}+9$ & 6.313 \\
\hline 2 & {$[2.05 \ldots 2.11]$} & 3.148 & $1.198 \mathrm{e}+9$ & 3.148 \\
\hline 3 & {$[2.89 \ldots 2.94]$} & 2.100 & $7.996 \mathrm{e}+8$ & 2.100 \\
\hline 4 & {$[3.09 \ldots 3.14]$} & 2.143 & $8.158 \mathrm{e}+8$ & 2.143 \\
\hline 5 & {$[3.96 \ldots 4.01]$} & 3.110 & $1.184 \mathrm{e}+9$ & 3.110 \\
\hline 6 & {$[6.61 \ldots 6.72]$} & 0.976 & $3.716 \mathrm{e}+8$ & 0.976 \\
\hline 7 & {$[6.86 \ldots 6.90]$} & 1.009 & $3.843 \mathrm{e}+8$ & 1.009 \\
\hline 8 & {$[8.02 \ldots 8.06]$} & 1.033 & $3.932 \mathrm{e}+8$ & 1.033 \\
\hline 9 & {$[8.07 \ldots 8.11]$} & 1.000 & $3.807 \mathrm{e}+8$ & 1.000 \\
\hline
\end{tabular}

\begin{tabular}{|c|c|c|c|c|c|c|c|c|c|}
\hline Multiplets Integrals Sun & 0.00 & Number of Nuclei & $7 \mathrm{~F}$ 's & & & & & & \\
\hline Acquisition Time (sec) & 0.6990 & Date & $2019 / 0$ & $8: 37: 15+0000$ & & Date Stamp & $2019 / 05 / 2$ & $8: 37: 15+0000$ & \\
\hline Frequency $(\mathrm{MHz})$ & 470.5077 & Nucleus & $19 \mathrm{~F}$ & Number of Transients & 64 & \begin{tabular}{|l|} 
Origin \\
\end{tabular} & li_z & Original Points Count & 65536 \\
\hline Owner & li_z & Points Count & 65536 & SW(cyclical) (Hz) & 93751.43 & Solvent & CHLOROP & RM-d & \\
\hline Spectrum Offset $(\mathrm{Hz})$ & -56467.0 & 0469 & & \begin{tabular}{|l|} 
Spectrum Type \\
\end{tabular} & undefined & Sweep Width $(\mathrm{Hz})$ & 93751.43 & Temperature (degree $C$ ) & 25.000 \\
\hline
\end{tabular}

${ }^{19} \mathrm{~F}$ NMR (471 MHz, CHLOROFORM- $d$ ) $\delta$ ppm -143.67 - -143.43 (m, 2 F) -141.57 - -141.27 (m, 2 F) -56.07 (t, J=21.46 Hz, 3 F)

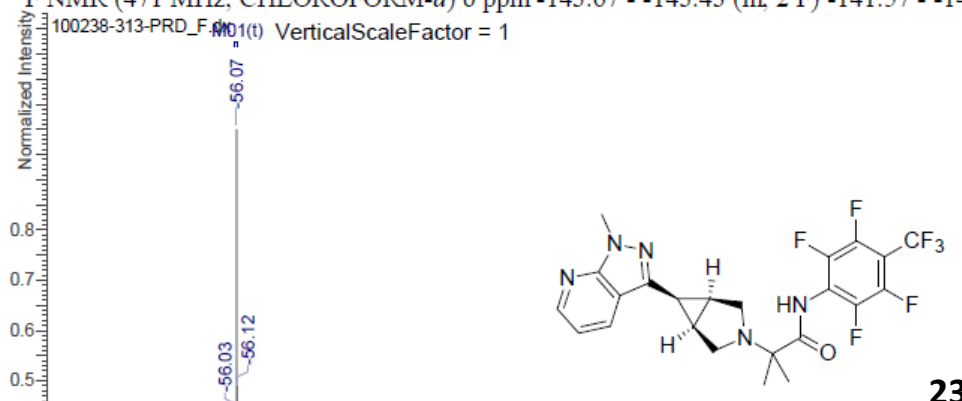

23

$\mathrm{MO2}(\mathrm{m})$ $\mathrm{MO}(\mathrm{m})$<smiles>C1=[As]2C[As]1[As]2</smiles>

$\frac{m^{\frac{1}{4}}}{\frac{\pi}{\tau}}$

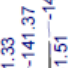

$\stackrel{+}{\longrightarrow}$

0

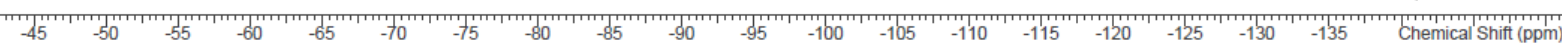

\begin{tabular}{|c|c|c|c|c|c|c|}
\hline No. & $\begin{array}{c}\text { Shift1 } \\
(\mathrm{ppm})\end{array}$ & F's & Type & $\begin{array}{c}\mathrm{J} \\
(\mathrm{Hz})\end{array}$ & Multiplet1 & $(\mathrm{ppm})$ \\
\hline 1 & -143.55 & 2 & $\mathrm{~m}$ & - & M03 & {$[-143.67 \ldots-143.43]$} \\
\hline 2 & -141.42 & 2 & $\mathrm{~m}$ & - & M02 & {$[-141.57 \ldots-141.27]$} \\
\hline 3 & -56.07 & 3 & $\mathrm{t}$ & 21.46 & M01 & {$[-56.17 \ldots-55.96]$} \\
\hline
\end{tabular}




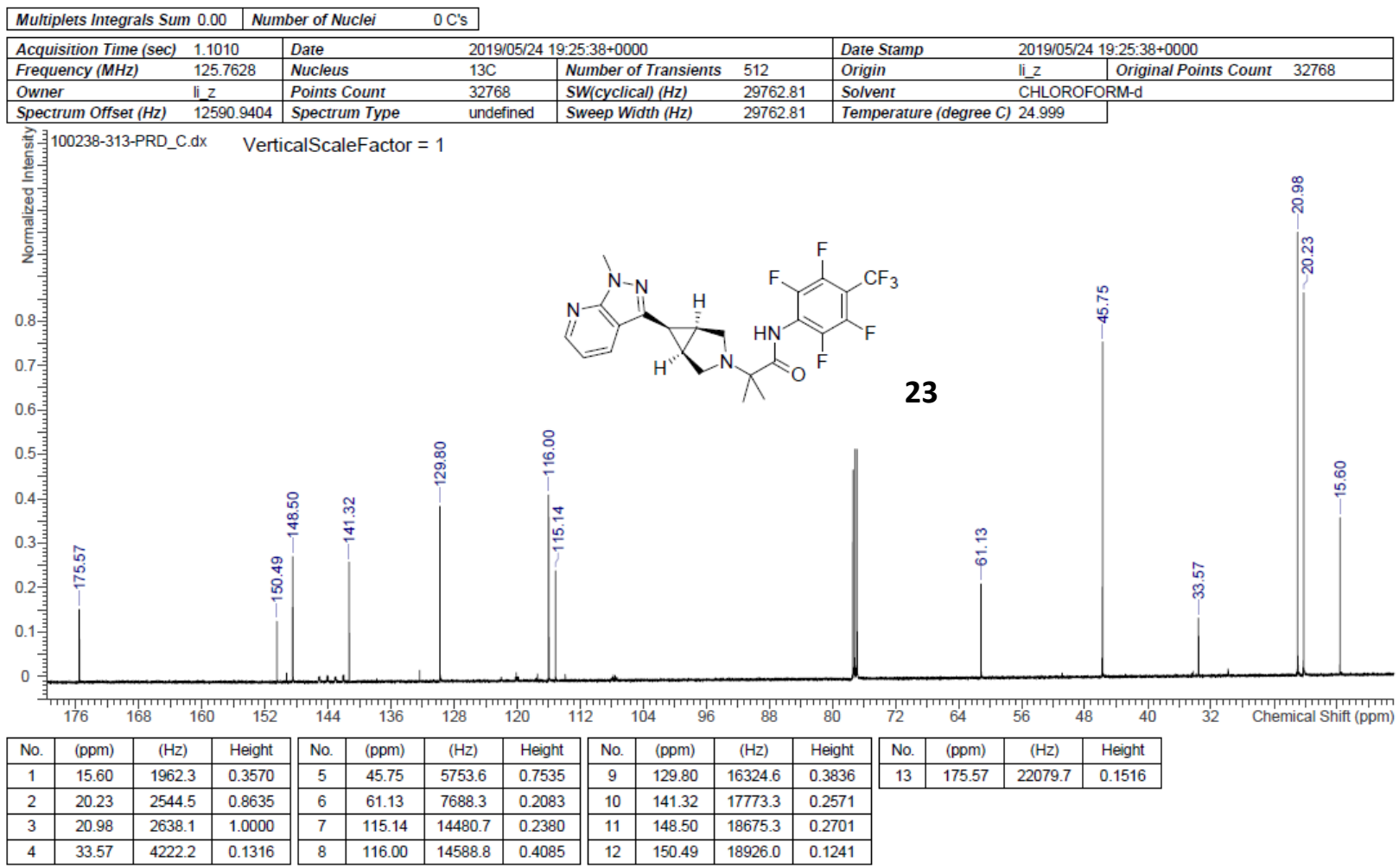

\begin{tabular}{|c|c|c|c|c|c|c|c|c|c|}
\hline Multiplets Integrals Sum & 24.34 & Number of Nuclei & $23 \mathrm{H}$ 's & & & & & & \\
\hline Acquisition Time (sec) & 1.6383 & Date & $2019 / 05$ & $13: 47: 36+0000$ & & Date Stamp & 2019 & $3: 47: 36+0000$ & \\
\hline Frequency $(\mathrm{MHz})$ & 500.1030 & Nucleus & $1 \mathrm{H}$ & Number of Transients & 8 & \begin{tabular}{|l} 
Origin \\
\end{tabular} & li_z & Original Points Coumt & 16384 \\
\hline Owner & li_z & Points Count & 16384 & SW(cyclical) $(\mathrm{Hz})$ & 10000.61 & Solvent & $\mathrm{CHL}$ & RM-d & \\
\hline
\end{tabular}

${ }^{1} \mathrm{H}$ NMR $(500 \mathrm{MHz}, \mathrm{CHLOROFORM}-d) \delta \mathrm{ppm} 1.07$ (s, $\left.6 \mathrm{H}\right) 2.06-2.17$ (m, $\left.3 \mathrm{H}\right) 2.90$ (br d, J=9.16 Hz, $\left.2 \mathrm{H}\right) 3.10$ (d, J=9.16 Hz, $\left.2 \mathrm{H}\right) 5.60$ (s, $\left.2 \mathrm{H}\right)$ $690-7.02(\mathrm{~m}, 4 \mathrm{H}) 7.16-7.23(\mathrm{~m}, 1 \mathrm{H}) 7.27$ (br s, $1 \mathrm{H}) 8.09(\mathrm{dd}, J=7.94,1.22 \mathrm{~Hz}, 1 \mathrm{H}) 8.21(\mathrm{dd}, J=4.58,1.53 \mathrm{~Hz}, 1 \mathrm{H})$

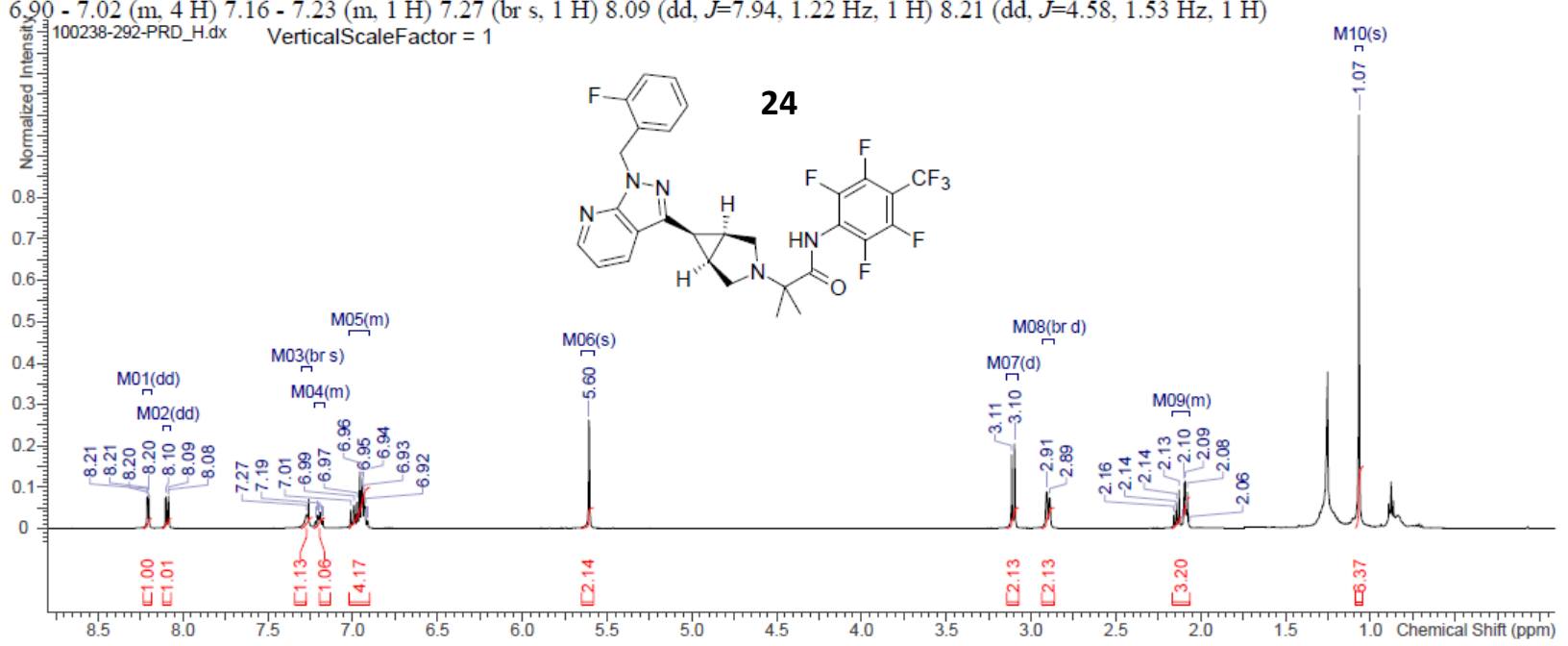

\begin{tabular}{|c|c|c|c|c|c|c|c|c|c|c|c|c|c|}
\hline No. & $\begin{array}{l}\text { Shift1 } \\
\text { (ppm) }\end{array}$ & H's & Type & $\begin{array}{c}\mathrm{J} \\
(\mathrm{Hz})\end{array}$ & Multiplet1 & (ppm) & No. & $\begin{array}{l}\text { Shift1 } \\
(\mathrm{ppm})\end{array}$ & H's & Type & $\begin{array}{c}\mathrm{J} \\
(\mathrm{Hz})\end{array}$ & Multiplet1 & (ppm) \\
\hline 1 & 1.07 & 6 & $\mathrm{~s}$ & - & M10 & {$[1.04 \ldots 1.09]$} & 6 & 6.95 & 4 & $\mathrm{~m}$ & - & M05 & {$\left[\begin{array}{ll}6.90 & . .7 \\
7.02\end{array}\right]$} \\
\hline 2 & 2.11 & 3 & $\mathrm{~m}$ & - & M09 & {$\left[\begin{array}{lll}2.06 & 2.17\end{array}\right]$} & 7 & 7.20 & 1 & $\mathrm{~m}$ & - & M04 & {$[7.16 \ldots 7.23]$} \\
\hline 3 & 2.90 & 2 & brd & 9.16 & M08 & {$\left[\begin{array}{lll}2.86 & .2 .94\end{array}\right]$} & 8 & 7.27 & 1 & brs & - & M03 & {$[7.24 \ldots . .7 .30]$} \\
\hline 4 & 3.10 & 2 & d & 9.16 & M07 & {$\left[\begin{array}{lll}3.07 & 3.14\end{array}\right]$} & 9 & 8.09 & 1 & dd & \begin{tabular}{|l|}
$7.94,1.22$ \\
\end{tabular} & M02 & {$\left[\begin{array}{lll}8.07 & . .8 .12\end{array}\right]$} \\
\hline 5 & 5.60 & 2 & $\mathrm{~s}$ & - & M06 & {$\left[\begin{array}{l}5.58 \\
. .5 .65]\end{array}\right.$} & 10 & 8.21 & 1 & dd & $4.58,1.53$ & M01 & {$[8.19 \ldots .8 .24]$} \\
\hline
\end{tabular}




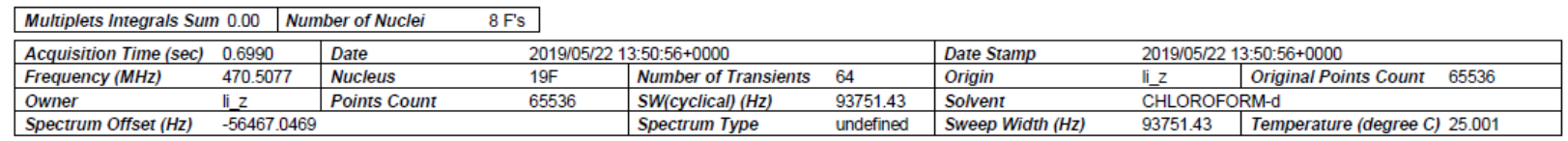

\begin{tabular}{lllll}
\hline${ }^{19} \mathrm{~F}$ NMR $(471 \mathrm{MHz}, \mathrm{CHLOROFORM-} d) \delta \mathrm{ppm}-143.05--142.73(\mathrm{~m}, 2 \mathrm{~F})-141.66--141.22(\mathrm{~m}, 2 \mathrm{~F})-118.49(\mathrm{~s}, 1 \mathrm{~F})-56.09(\mathrm{t}, \mathrm{J}=22.17 \mathrm{~Hz}, 3 \mathrm{~F})$
\end{tabular}

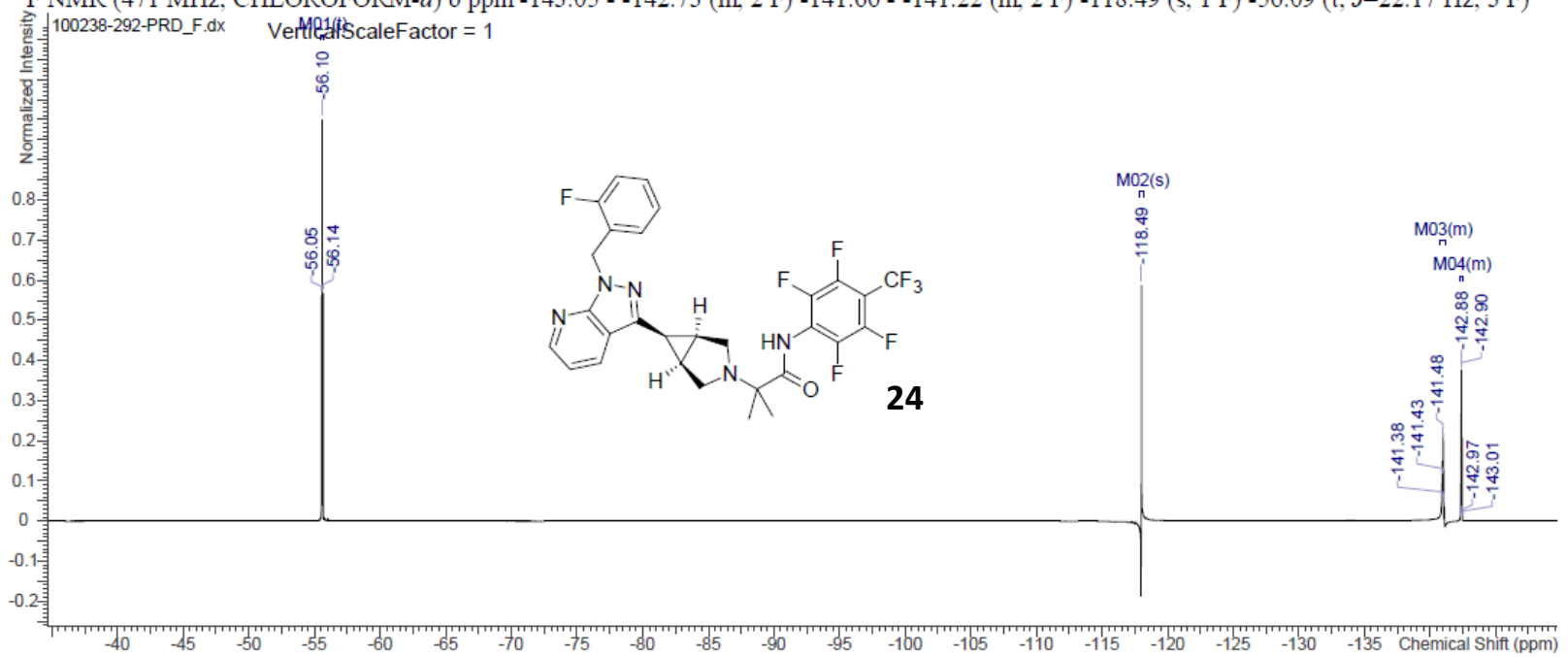

\begin{tabular}{|c|c|c|c|c|c|c|}
\hline No. & $\begin{array}{c}\text { Shift1 } \\
(\mathrm{ppm})\end{array}$ & $\mathrm{F}$ 's & Type & $\begin{array}{c}\mathrm{J} \\
(\mathrm{Hz})\end{array}$ & Multiplet1 & $(\mathrm{ppm})$ \\
\hline 1 & -142.89 & 2 & $\mathrm{~m}$ & - & $\mathrm{M} 04$ & {$[-143.05 \ldots-142.73]$} \\
\hline 2 & -141.47 & 2 & $\mathrm{~m}$ & - & $\mathrm{M} 03$ & {$[-141.66 \ldots-141.22]$} \\
\hline 3 & -118.49 & 1 & $\mathrm{~s}$ & - & $\mathrm{M} 02$ & {$[-118.65 \ldots-118.39]$} \\
\hline 4 & -56.09 & 3 & $\mathrm{t}$ & 22.17 & $\mathrm{M} 01$ & {$[-56.20 \ldots-55.97]$} \\
\hline
\end{tabular}

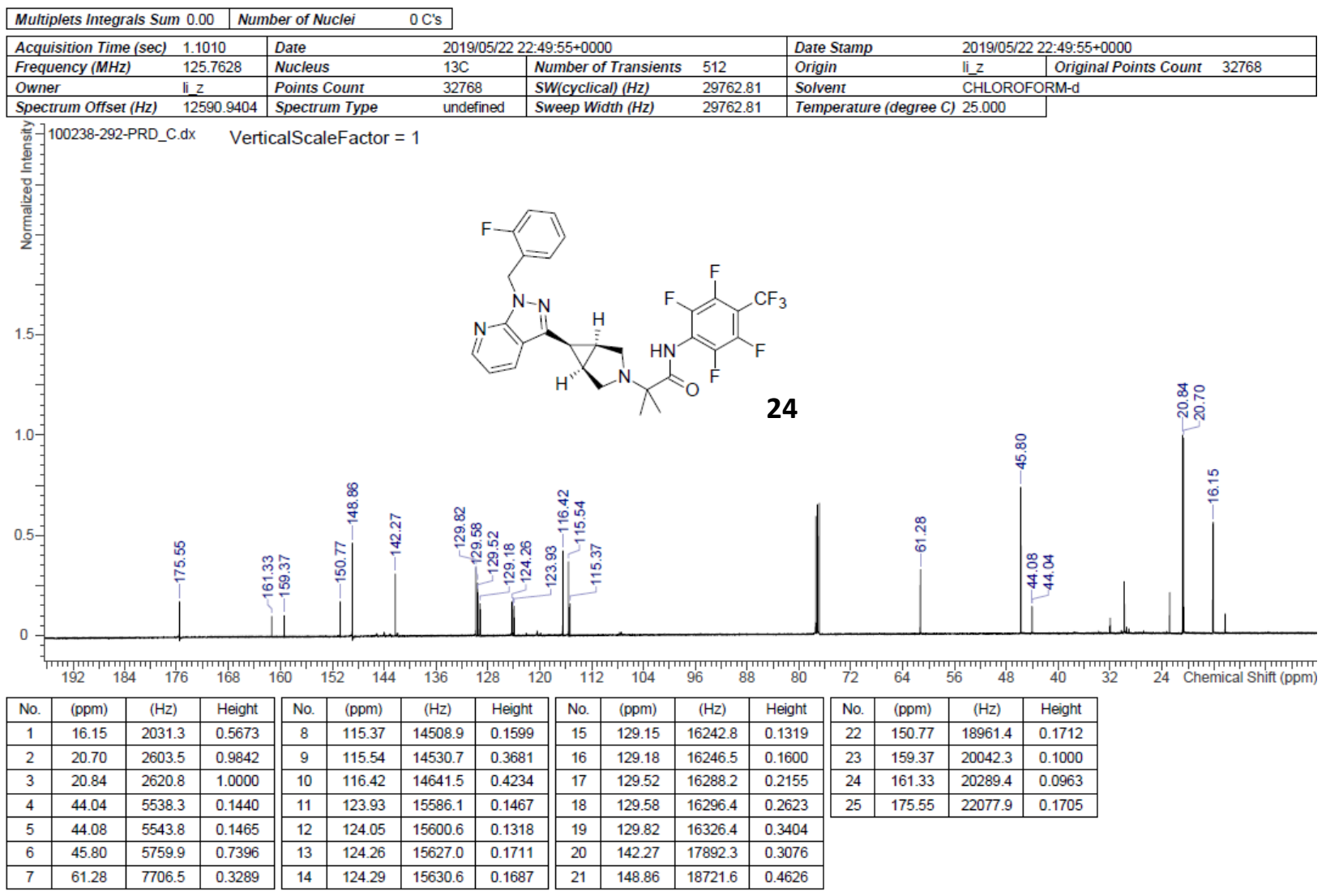




\begin{tabular}{|c|c|c|c|c|c|c|c|c|c|}
\hline Multiplets Integrals Sum & 18.61 & Number of Nuclei & $18 \mathrm{H}$ 's & & & & & & \\
\hline Acquisition Time (sec) & 1.6383 & Date & $2019 / 05$ & $21: 59: 47+0000$ & & Date Stamp & 2019 & $1: 59: 47+0000$ & \\
\hline Frequency $(\mathrm{MHz})$ & 500.1030 & Nucleus & $1 \mathrm{H}$ & Number of Transients & 8 & Origin & li_z & Original Points Count & 16384 \\
\hline Owner & li_z & Points Count & 16384 & SW(cyclical) (Hz) & 10000.61 & Solvent & $\mathrm{CHL}$ & RM-d & \\
\hline
\end{tabular}

undefined Sweep Width (Hz) 1000061 Temperature (degree C) 25.000

${ }^{1} \mathrm{H}$ NMR $(500 \mathrm{MHz}$, CHLOROFORM- $d$ ) $\delta \mathrm{ppm} 1.14(\mathrm{~s}, 6 \mathrm{H}) 1.98-2.03(\mathrm{~m}, 2 \mathrm{H}) 2.12(\mathrm{t}, J=8.55 \mathrm{~Hz}, 1 \mathrm{H}) 2.93-3.04$ (m, $4 \mathrm{H}) 6.56$ (br s, $\left.1 \mathrm{H}\right) 7.56$ (dd, $J=9.16,1.22 \mathrm{~Hz}, 1 \mathrm{H}) 7.62(\mathrm{~d}, J=9.16 \mathrm{~Hz}, 1 \mathrm{H}) 7.98(\mathrm{~s}, 1 \mathrm{H}) 8.51(\mathrm{~s}, 1 \mathrm{H})$



\begin{tabular}{|c|c|c|c|c|c|c|c|c|}
\hline \multicolumn{3}{|c|}{\begin{tabular}{|l|l|l} 
Uultiplets Integrals Sum 0.00 & Number of Nuclei
\end{tabular}} & \multicolumn{6}{|c|}{ F's } \\
\hline Acquisition Time (sec) & 0.6990 & Date & $2019 / 0$ & 2:03:48+0000 & & Date Stamp & $2019 / 05 / 23$ & $22: 03: 48+0000$ \\
\hline Frequency $(\mathrm{MHz})$ & 470.5077 & Nucleus & $19 \mathrm{~F}$ & Number of Transients & 64 & Origin & li_z & Original Points Count \\
\hline Owner & lijz & Points Count & 65536 & $S W_{(\text {(cyclical) }(H z)}$ & 93751.43 & Solvent & CHLOROF & RM-d \\
\hline Spectrum Offset (Hz) & -56467.046 & & & Spectrum Type & undefined & Sweep Width $(\mathrm{Hz})$ & 93751.43 & Temperature (degree C) 25.000 \\
\hline
\end{tabular}

${ }^{19} \mathrm{~F}$ NMR (471 MHz, CHLOROFORM- $d$ ) $\delta \mathrm{ppm}-143.76--143.54$ (m, 2 F) $-140.82--140.50$ (m, 2 F) -56.15 (t, J=21.46 Hz, 3 F)

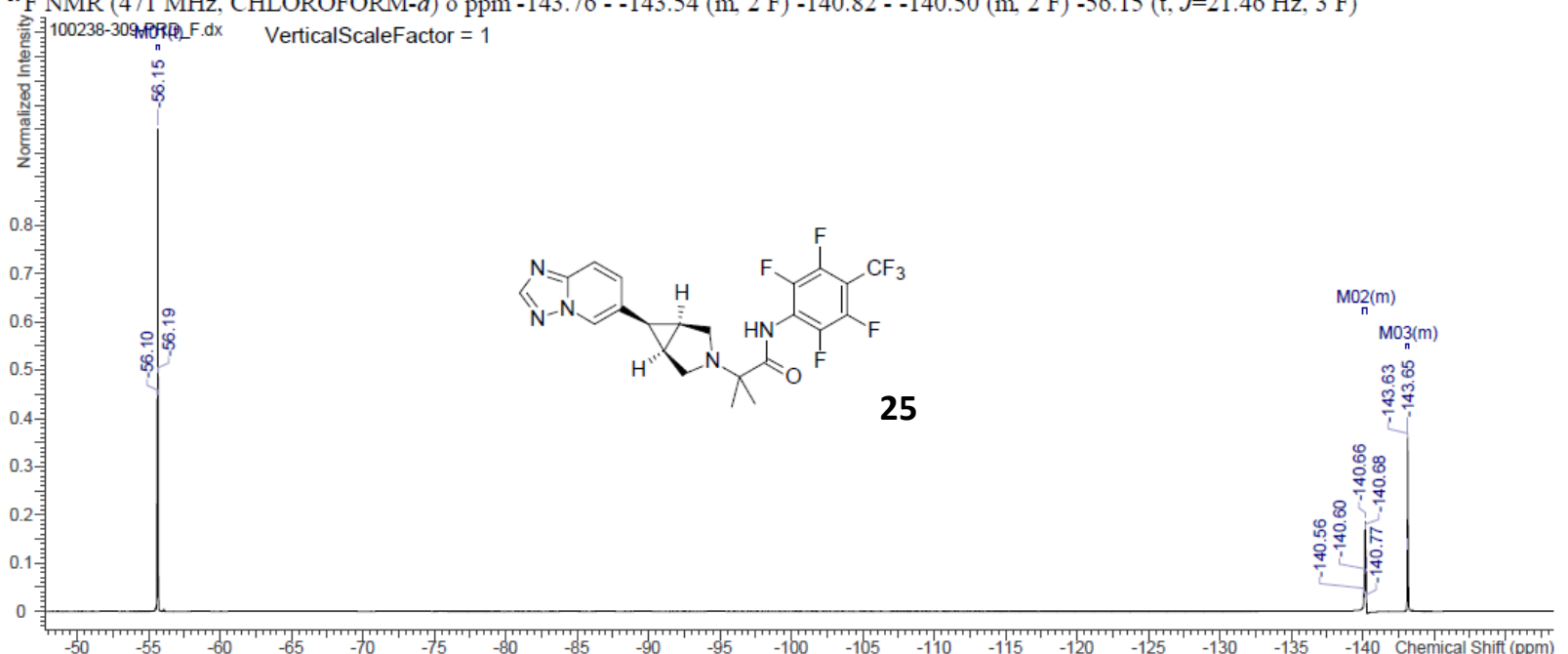

\begin{tabular}{|c|c|c|c|c|c|c|}
\hline No. & $\begin{array}{c}\text { Shift1 } \\
(\mathrm{ppm})\end{array}$ & F's & Type & $\begin{array}{c}\text { J } \\
(\mathrm{Hz})\end{array}$ & Multiplet1 & (ppm) \\
\hline 1 & -143.64 & 2 & $\mathrm{~m}$ & - & M03 & {$[-143.76 \ldots-143.54]$} \\
\hline 2 & -140.66 & 2 & $\mathrm{~m}$ & - & M02 & {$[-140.82 \ldots-140.50]$} \\
\hline 3 & -56.15 & 3 & $\mathrm{t}$ & 21.46 & M01 & {$[-56.25 \ldots-.56 .04]$} \\
\hline
\end{tabular}




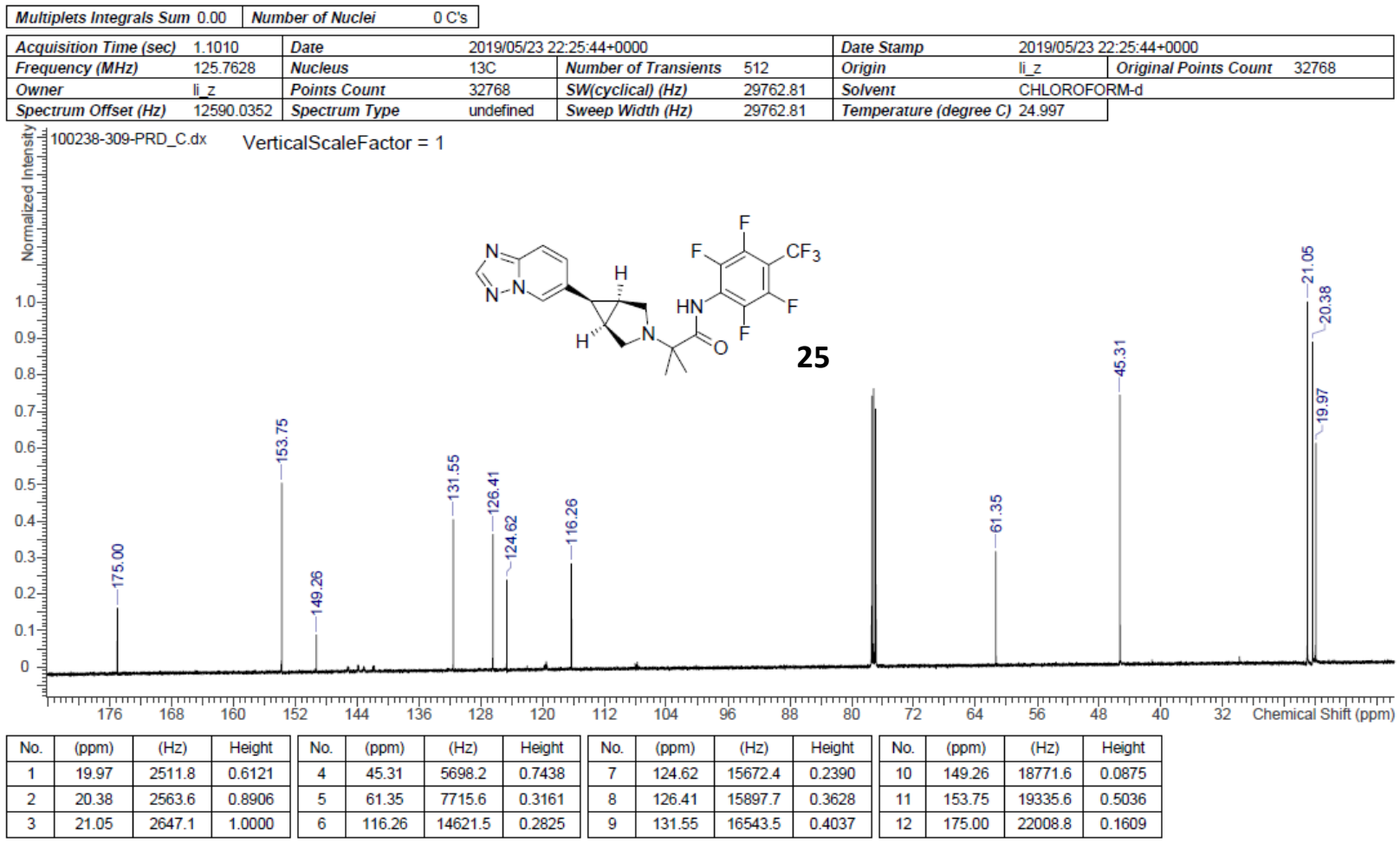

\begin{tabular}{|l|lr|}
\hline Multiplets Integrals Sum 18.18 & Number of Nuclei $18 \mathrm{H}$ 's \\
\hline
\end{tabular}

\begin{tabular}{|c|c|c|c|c|c|c|c|c|c|}
\hline Acquisition Time (sec) & 1.6383 & Date & $2019 / 05 / 24$ & $8: 23: 26+0000$ & & Date Stamp & $2019 / 05 /$ & $18: 23: 26+0000$ & \\
\hline Frequency $(\mathrm{MHz})$ & 500.1030 & Nucleus & $1 \mathrm{H}$ & Number of Transients & 8 & Origin & li_z & Original Points Count & 16384 \\
\hline Owner & li_z & Points Count & 16384 & $S W($ cyclical $)(\mathrm{Hz})$ & 10000.61 & Solvent & CHLORC & RM-d & \\
\hline Spectrum Offset (Hz) & 2987.6548 & Spectrum Type & undefined & Sweep Width $(\mathrm{Hz})$ & 10000.61 & Temperature (degree C) & 25.001 & & \\
\hline
\end{tabular}

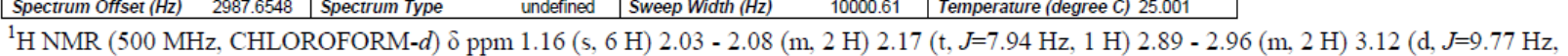

$2 \mathrm{H}) 6.56(\mathrm{br} \mathrm{s}, 1 \mathrm{H}) 7.10(\mathrm{~d}, J=9.16 \mathrm{~Hz}, 1 \mathrm{H}) 7.37(\mathrm{~s}, 1 \mathrm{H}) 7.67(\mathrm{~s}, 1 \mathrm{H}) 7.83(\mathrm{~d}, J=9.16 \mathrm{~Hz}, 1 \mathrm{H})$

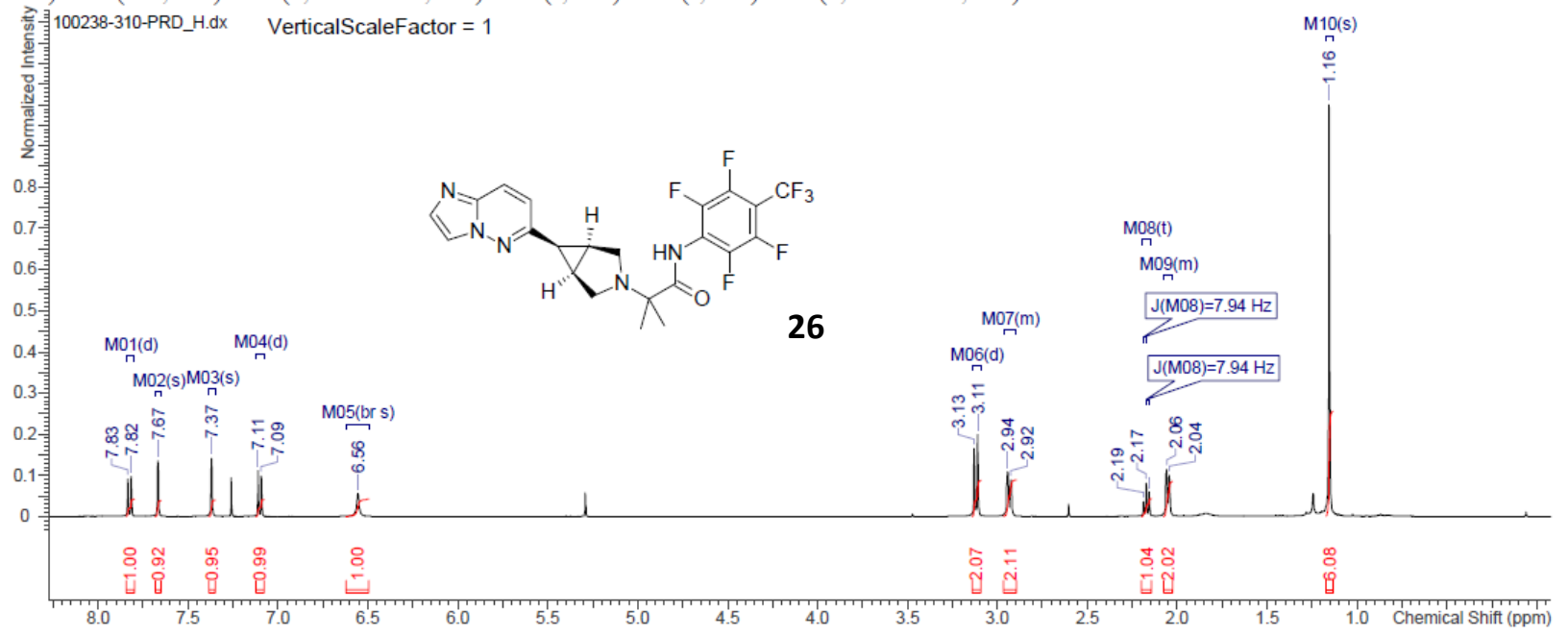

\begin{tabular}{|c|c|c|c|c|c|c|c|c|c|c|c|c|c|}
\hline No. & $\begin{array}{l}\text { Shift1 } \\
(\mathrm{ppm})\end{array}$ & $\mathrm{H}$ 's & Type & $\begin{array}{c}J \\
(\mathrm{~Hz})\end{array}$ & Multiplet1 & (ppm) & No. & $\begin{array}{l}\text { Shift1 } \\
\text { (ppm) }\end{array}$ & H's & Type & $\underset{(\mathrm{Hz})}{\mathrm{J}}$ & Multiplet1 & (ppm) \\
\hline 1 & 1.16 & 6 & $\mathrm{~s}$ & - & M10 & {$\left[\begin{array}{llll}1.14 & \ldots & 1.17]\end{array}\right.$} & 6 & 6.56 & 1 & $\mathrm{brs}$ & - & M05 & {$[6.50 \ldots 6.62]$} \\
\hline 2 & 2.06 & 2 & $\mathrm{~m}$ & - & M09 & {$\left[\begin{array}{lll}2.03 & .2 .08\end{array}\right]$} & 7 & 7.10 & 1 & d & 9.16 & M04 & {$[7.08 \ldots 7.12]$} \\
\hline 3 & 2.17 & 1 & $t$ & 7.94 & M08 & {$\left[\begin{array}{llll}2.14 & .2 .20]\end{array}\right]$} & 8 & 7.37 & 1 & $\mathrm{~s}$ & - & M03 & {$[7.35 \ldots 7.38]$} \\
\hline 4 & 2.93 & 2 & $\mathrm{~m}$ & - & M07 & {$[2.89 \ldots 2.96]$} & 9 & 7.67 & 1 & $\mathrm{~s}$ & - & M02 & {$[7.65 \ldots 7.68]$} \\
\hline 5 & 3.12 & 2 & d & 9.77 & M06 & {$\left[\begin{array}{lll}3.09 & . & 3.14\end{array}\right]$} & 10 & 7.83 & 1 & $d$ & 9.16 & M01 & {$[7.80 \ldots 7.84]$} \\
\hline
\end{tabular}




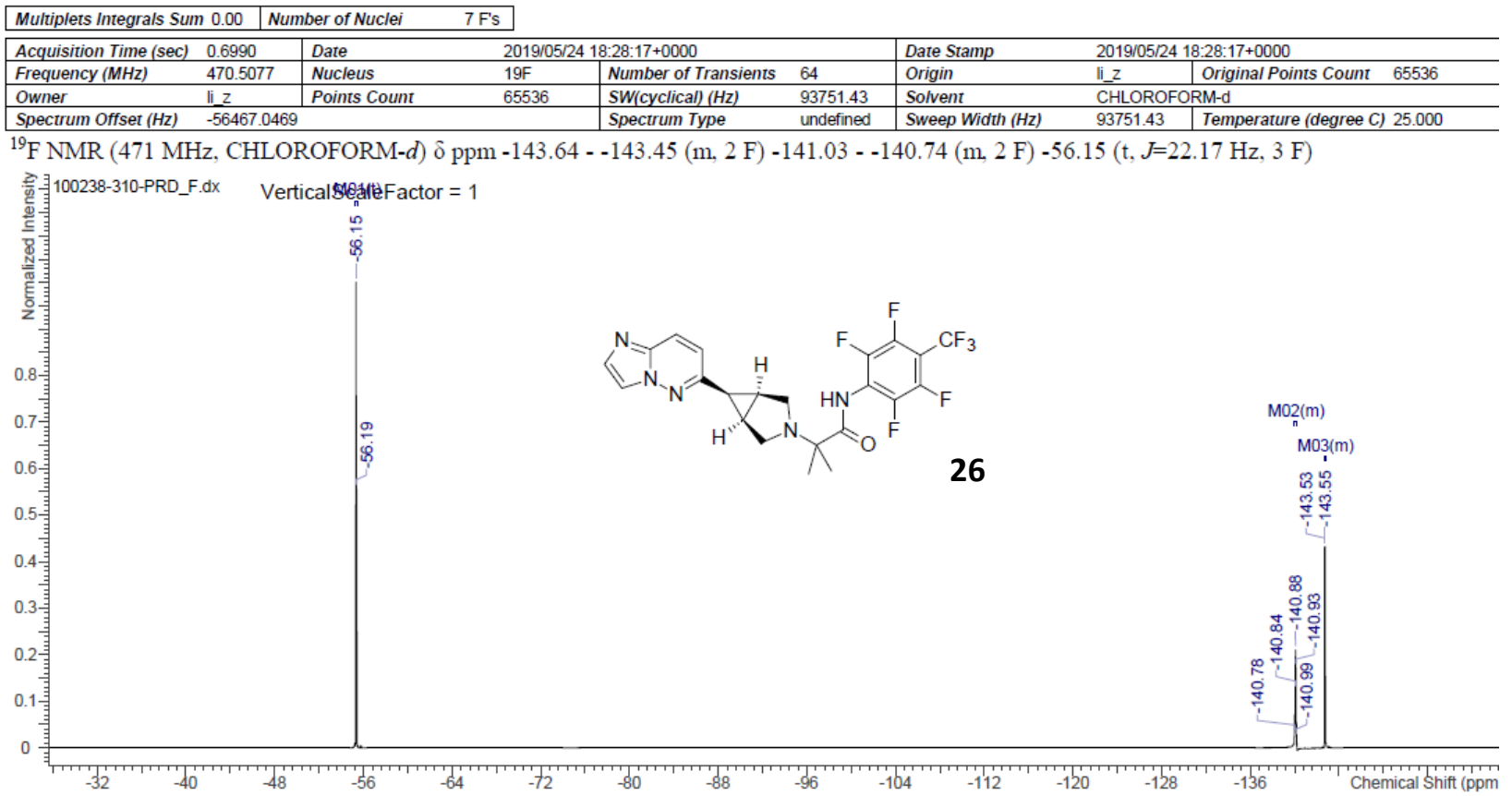

\begin{tabular}{|c|c|c|c|c|c|c|}
\hline No. & $\begin{array}{c}\text { Shift1 } \\
(\mathrm{ppm})\end{array}$ & F's & Type & $\begin{array}{c}\text { J } \\
(\mathrm{Hz})\end{array}$ & Multiplet1 & $(\mathrm{ppm})$ \\
\hline 1 & -143.54 & 2 & $\mathrm{~m}$ & - & $\mathrm{M} 03$ & {$[-143.64 \ldots-143.45]$} \\
\hline 2 & -140.89 & 2 & $\mathrm{~m}$ & - & $\mathrm{M} 02$ & {$[-141.03 \ldots-140.74]$} \\
\hline 3 & -56.15 & 3 & $\mathrm{t}$ & 22.17 & M01 & {$[-56.26 \ldots-56.05]$} \\
\hline
\end{tabular}

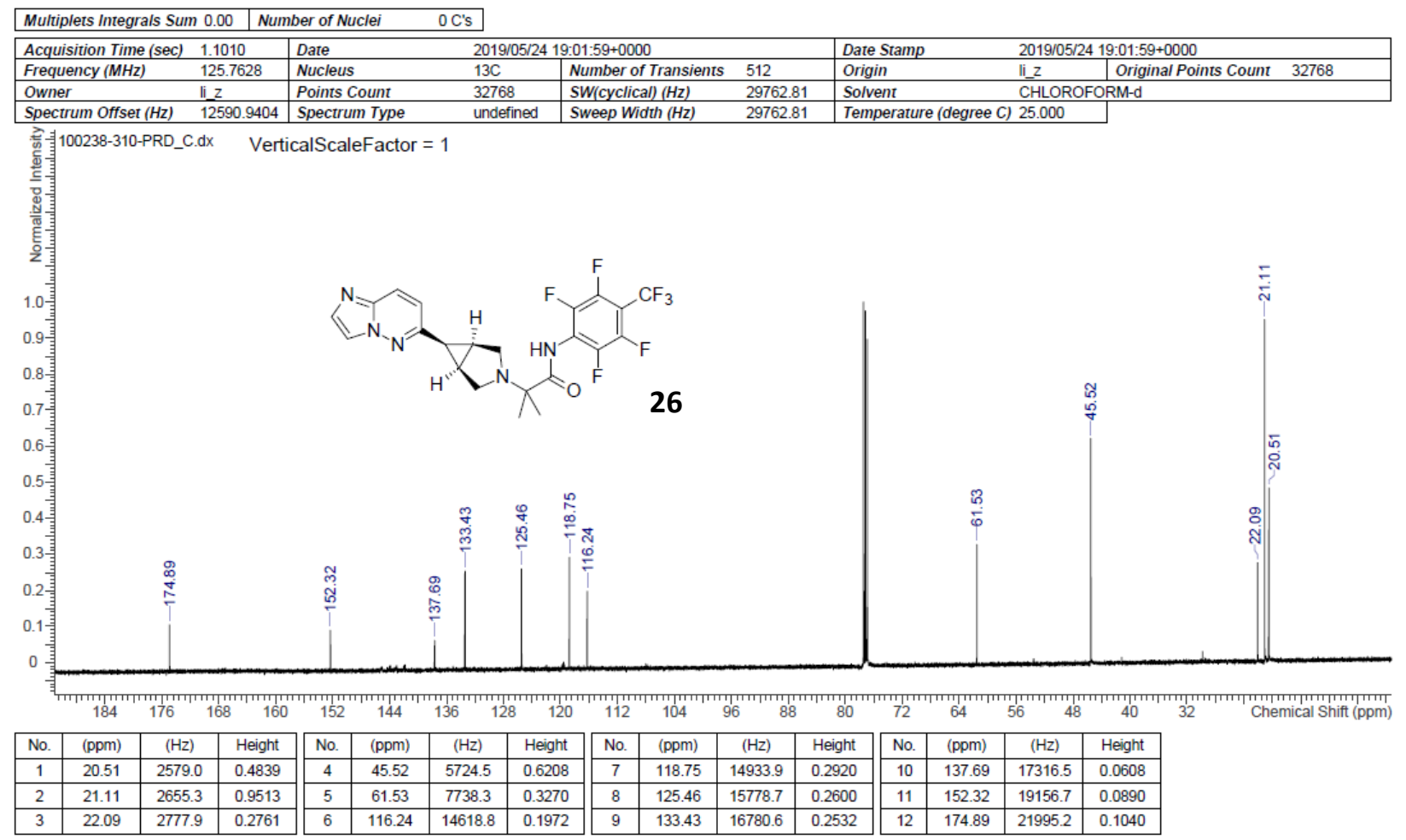




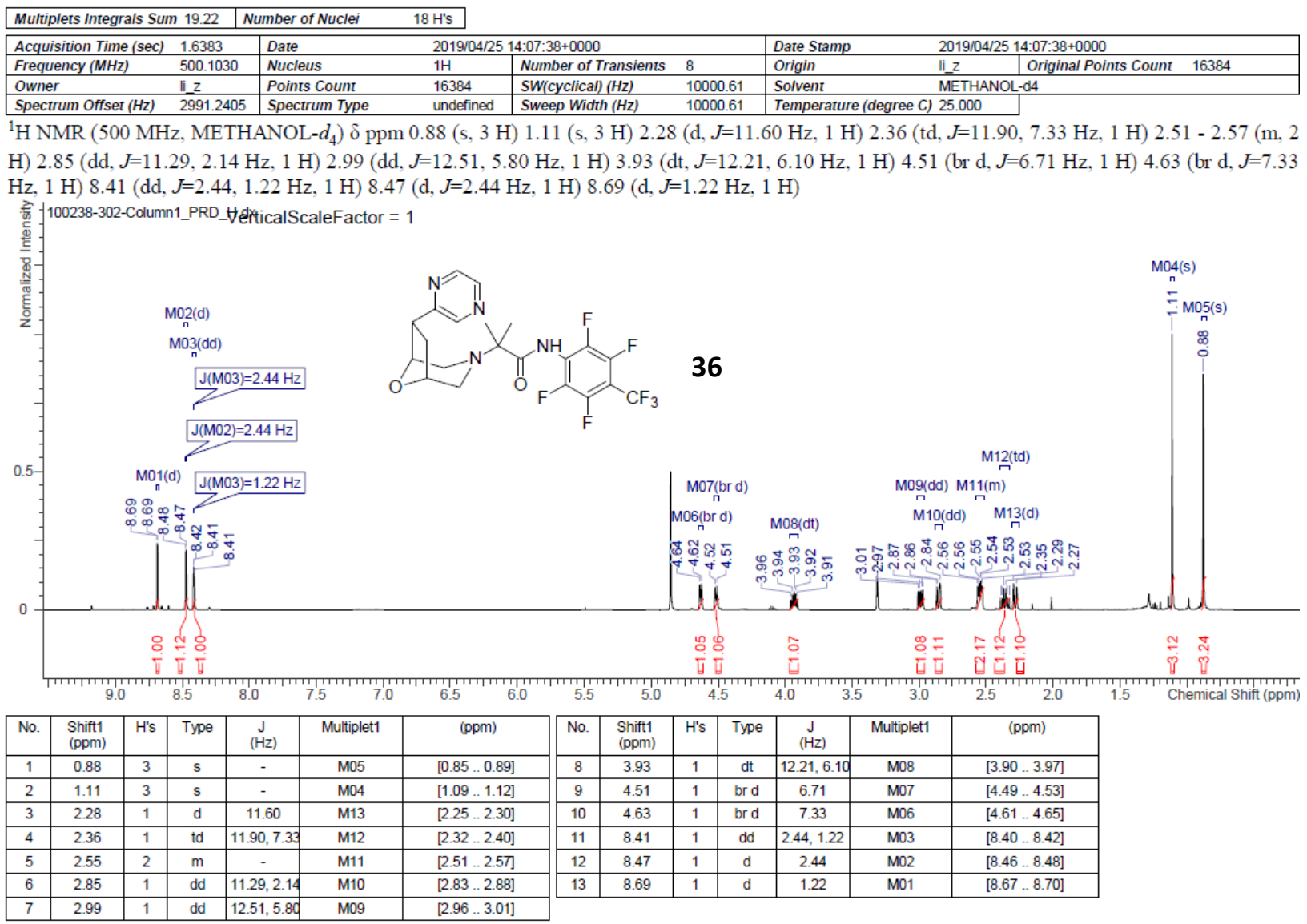

\begin{tabular}{|c|c|c|c|c|c|c|c|c|c|}
\hline \multicolumn{2}{|c|}{ Multiplets Integrals Sum 0.00} & Number of Nuclei & \multirow{2}{*}{\multicolumn{3}{|c|}{$7 \mathrm{Fs}$}} & \multirow[b]{2}{*}{ Date Stamp } & \multirow{2}{*}{\multicolumn{3}{|c|}{$2019 / 04 / 25$ 14:11:25+0000 }} \\
\hline Acquisition Time (sec) & 0.6990 & Date & & & & & & & \\
\hline Frequency $(\mathrm{MHz})$ & 470.5077 & Nucleus & $19 \mathrm{~F}$ & Number of Transients & 64 & Origin & li_z & Original Points Count & 65536 \\
\hline Owner & li_z & Points Count & 65536 & SW(cyclical) (Hz) & 93751.43 & Solvent & METHANC & & \\
\hline Spectrum Offset $(\mathrm{Hz})$ & -56467.0 & 0469 & & Spectrum Type & undefined & Sweep Width $(\mathrm{Hz})$ & 93751.43 & Temperature (degree C & 24.999 \\
\hline
\end{tabular}

${ }^{19} \mathrm{~F}$ NMR $\left(471 \mathrm{MHz}\right.$, METHANOL- $\left.d_{4}\right) \delta \mathrm{ppm}-145.16$ - -144.99 (m, 2 F) -144.07 - -143.79 (m, 2 F) -57.56 (t, $J=21.46$ Hz, 3 F)

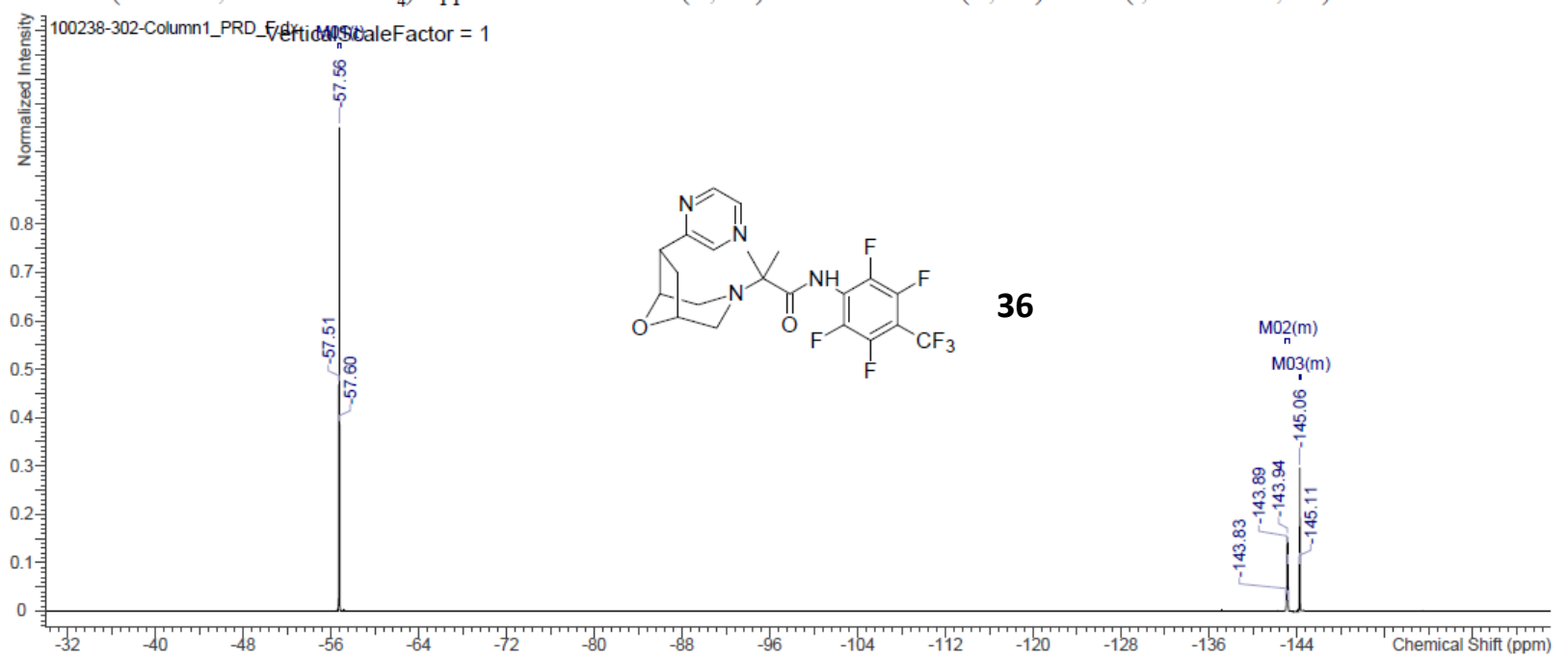

\begin{tabular}{|c|c|c|c|c|c|c|}
\hline No. & $\begin{array}{c}\text { Shift1 } \\
(\mathrm{ppm})\end{array}$ & F's & Type & $\begin{array}{c}\mathrm{J} \\
(\mathrm{Hz})\end{array}$ & Multiplet1 & $(\mathrm{ppm})$ \\
\hline 1 & -145.07 & 2 & $\mathrm{~m}$ & - & $\mathrm{M} 03$ & {$[-145.16 \ldots-144.99]$} \\
\hline 2 & -143.92 & 2 & $\mathrm{~m}$ & - & $\mathrm{M} 02$ & {$[-144.07 \ldots-143.79]$} \\
\hline 3 & -57.56 & 3 & $\mathrm{t}$ & 21.46 & M01 & {$[-57.66 \ldots-57.46]$}
\end{tabular}




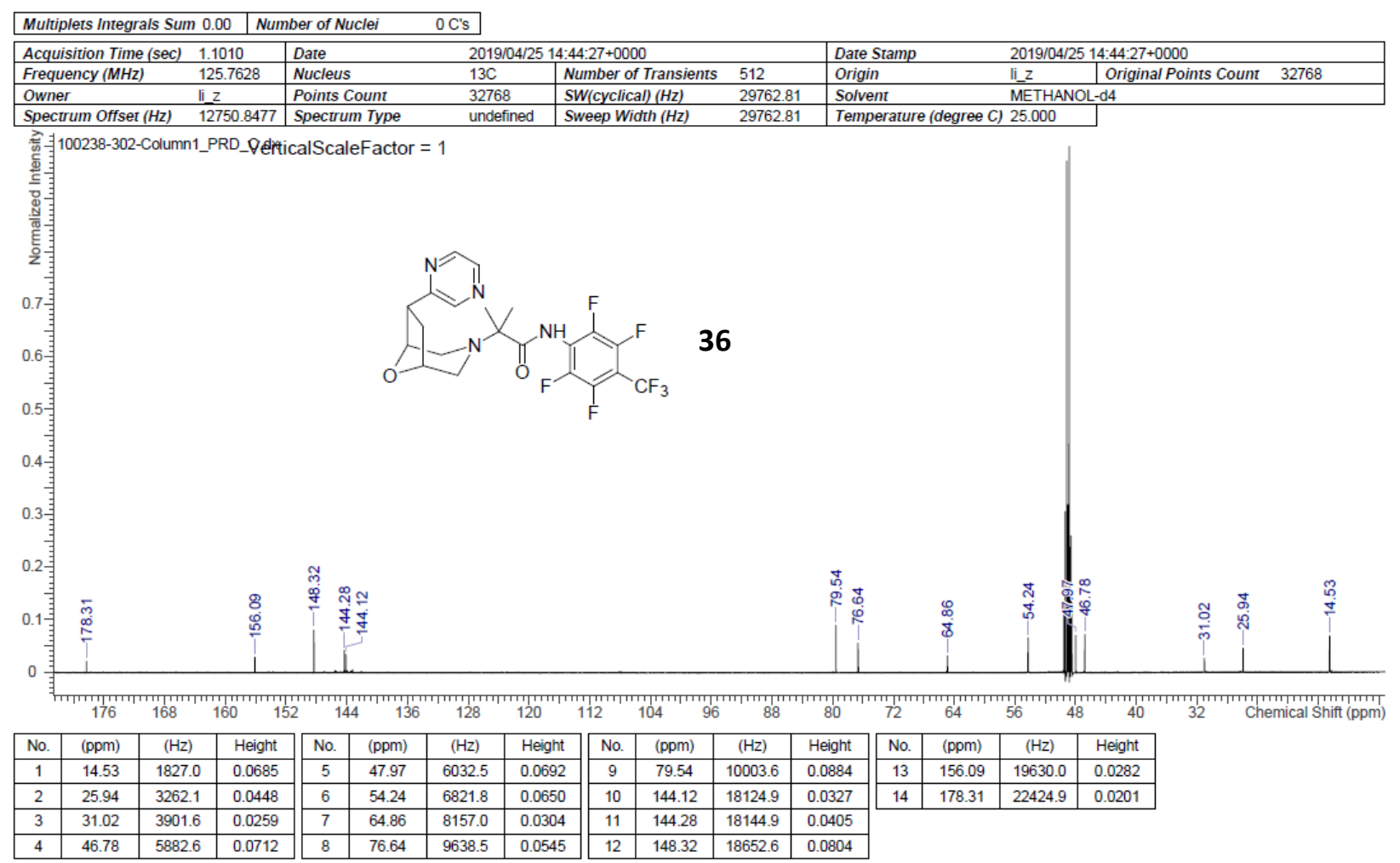

\begin{tabular}{|c|c|c|c|c|c|c|c|c|c|}
\hline Multiplets Integrals Sun & 20.18 & imber of Nuclei & $19 \mathrm{H}$ 's & & & & & & \\
\hline Acquisition Time (sec) & 1.6383 & \begin{tabular}{|l|} 
Date \\
\end{tabular} & $2019 / 05 / 29$ & $0: 15: 33+0000$ & & Date Stamp & $2019 / 05 /$ & 0:15:33+0000 & \\
\hline Frequency $(\mathrm{MHz})$ & 500.1030 & Nucleus & $1 \mathrm{H}$ & Number of Transients & 8 & Origin & li_z & \begin{tabular}{|l|} 
Original Points Count \\
\end{tabular} & 16384 \\
\hline Owner & li_z & Points Count & 16384 & SW(cyclical) $(\mathrm{Hz})$ & 10000.61 & Solvent & CHLORC & RM-d & \\
\hline Spectrum Offset $(\mathrm{Hz})$ & 2988.8704 & Spectrum Type & undefined & Sweep Width $(\mathrm{Hz})$ & 10000.61 & Temperature (degree $C$ ) & 25.000 & & \\
\hline
\end{tabular}

${ }^{1} \mathrm{H}$ NMR (500 MHz, CHLOROFORM-d) $\delta \mathrm{ppm} 1.17$ (s, $\left.6 \mathrm{H}\right) 2.68(\mathrm{dd}, J=11.60,3.66 \mathrm{~Hz}, 2 \mathrm{H}) 3.30$ (d, $\left.J=11.60 \mathrm{~Hz}, 2 \mathrm{H}\right) 3.68(\mathrm{t}, J=3.66 \mathrm{~Hz}, 2 \mathrm{H})$ $3.84(\mathrm{t}, J=4.27 \mathrm{~Hz}, 1 \mathrm{H}) 7.17(\mathrm{dd}, J=5.49,3.05 \mathrm{~Hz}, 2 \mathrm{H}) 7.27-7.30(\mathrm{~m}, 2 \mathrm{H}) 7.49($ br s, $1 \mathrm{H}) 7.79(\mathrm{~s}, 1 \mathrm{H})$
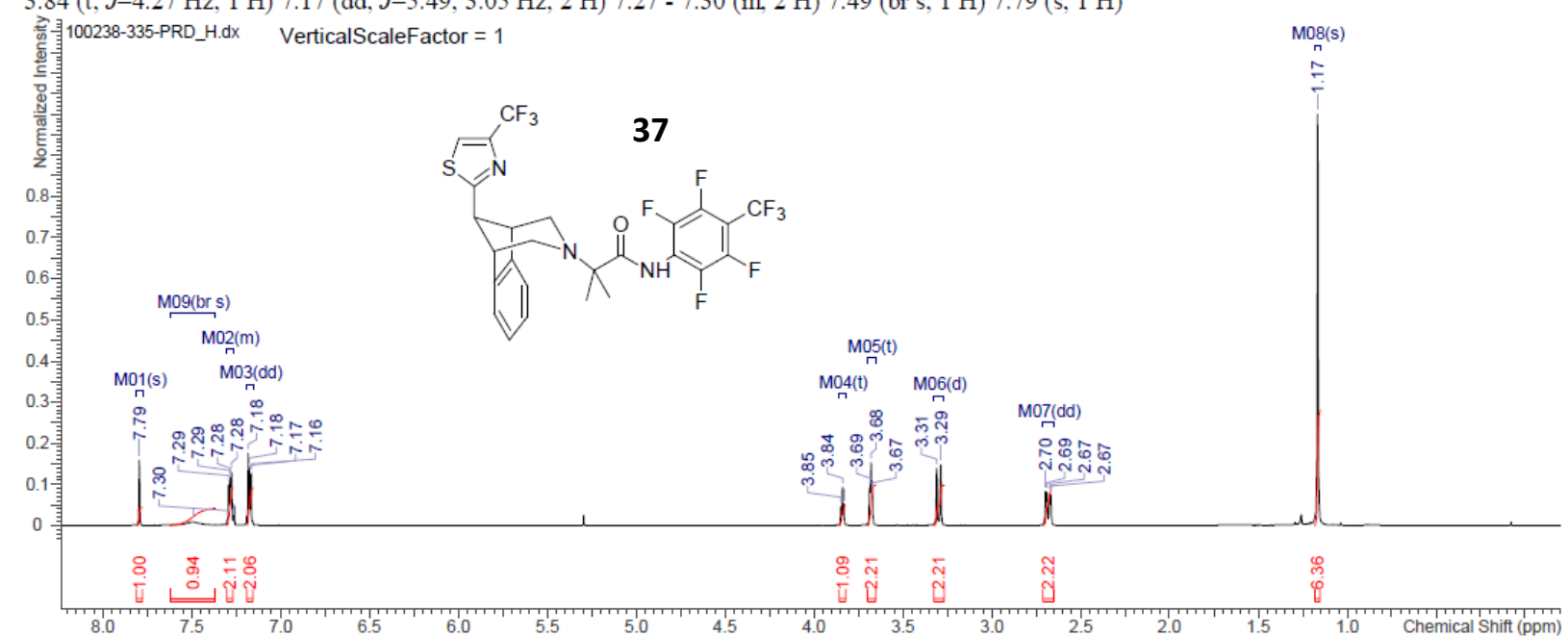

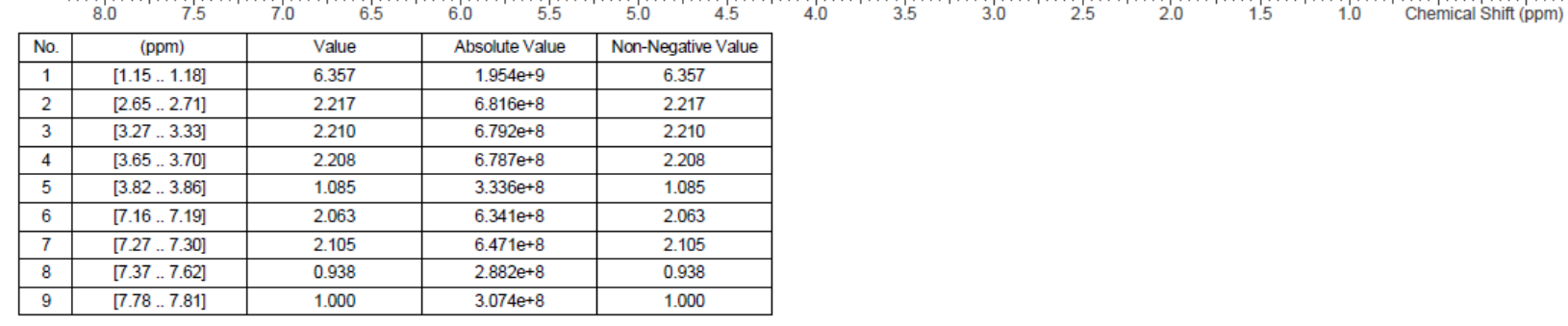




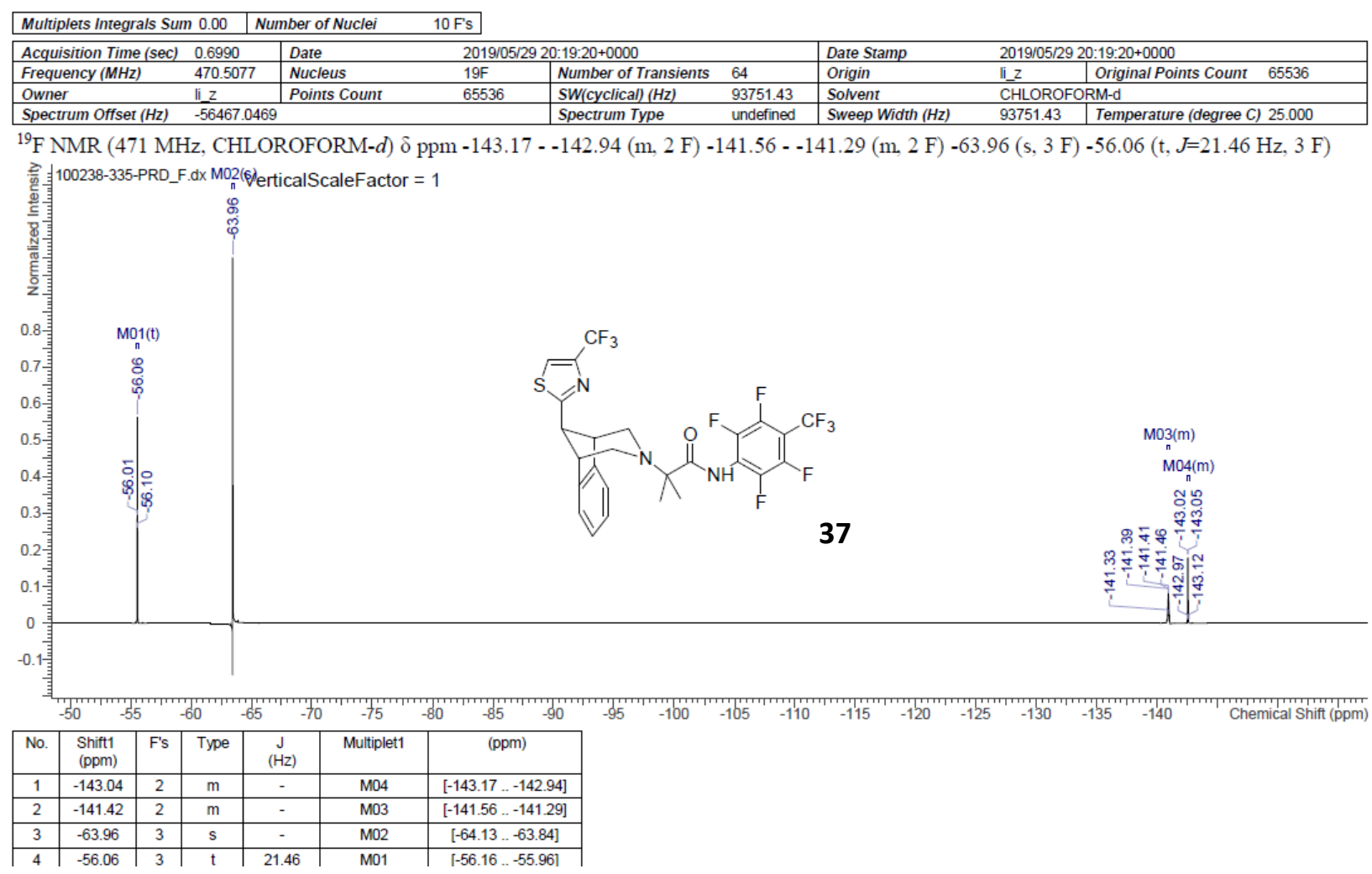

\begin{tabular}{|ll|ll|ll|l|l|}
\hline Multiplets Integrals Sum 0.00 & Number of Nuclei & OC's \\
\hline Acquisition Time $(\mathrm{sec})$ & 1.1010 & Date & 2019/05/29 22:24:14+0000 & Date Stamp & 2019/05/29 22:24:14+0000 \\
\hline Frequency $(\mathrm{MHz})$ & 125.7628 & Nucleus & $13 \mathrm{C}$ & Number of Transients & 512 & Origin & li_z \\
\hline Owner & li_z & Points Count & 32768 & SW(cyclical) $(\mathrm{Hz})$ & 29762.81 & Solvent & CHLOROFORM-d \\
\hline Spectrum Offset $(\mathrm{Hz})$ & 12590.9404 & Spectrum Type & undefined & Sweep Width $(\mathrm{Hz})$ & 29762.81 & Temperature (degree C) 25.000 & 32768 \\
\hline
\end{tabular}

空聿100238-335-PRD_C.dx VerticalScaleFactor =

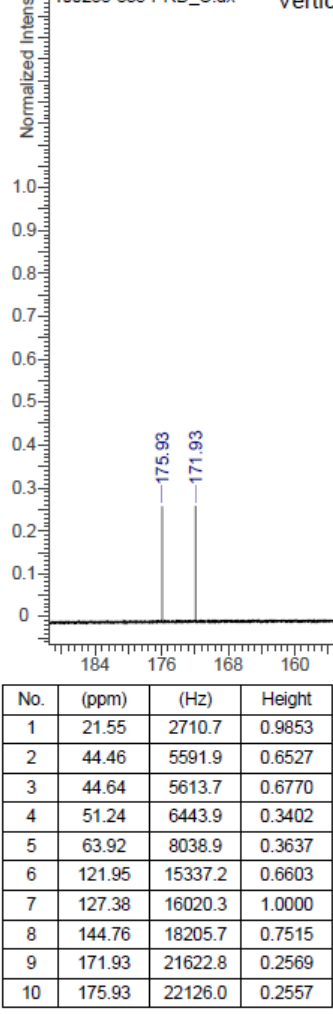



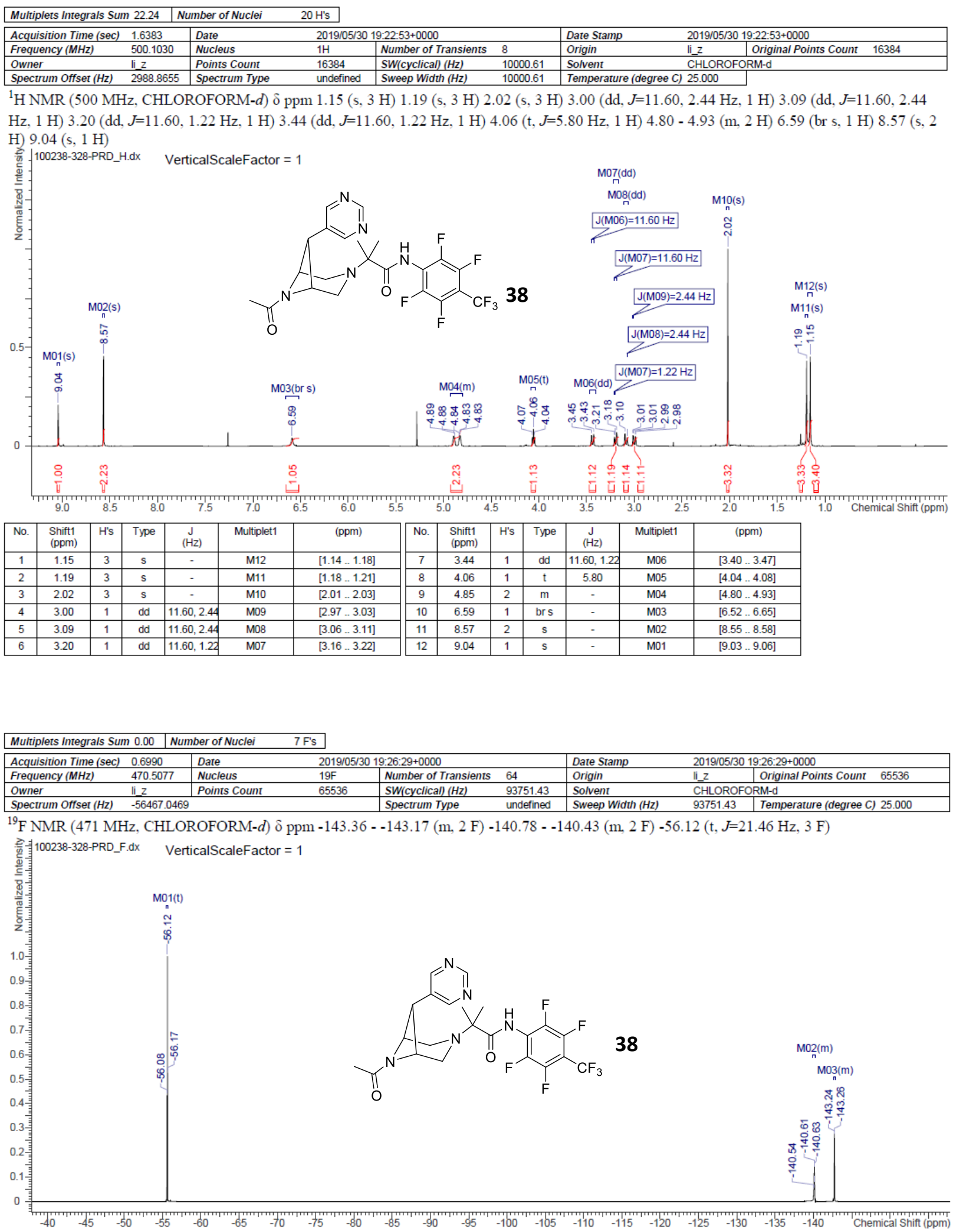

\begin{tabular}{|c|c|c|c|c|c|c|}
\hline No. & $\begin{array}{c}\text { Shift1 } \\
(\mathrm{ppm})\end{array}$ & F's & Type & $\begin{array}{c}\mathrm{J} \\
(\mathrm{Hz})\end{array}$ & Multiplet1 & $(\mathrm{ppm})$ \\
\hline 1 & -143.25 & 2 & $\mathrm{~m}$ & - & M03 & {$[-143.36 \ldots-143.17]$} \\
\hline 2 & -140.62 & 2 & $\mathrm{~m}$ & - & M02 & {$[-140.78 \ldots-140.43]$} \\
\hline 3 & -56.12 & 3 & $\mathrm{t}$ & 21.46 & M01 & {$[-56.23 \ldots-56.02]$} \\
\hline
\end{tabular}




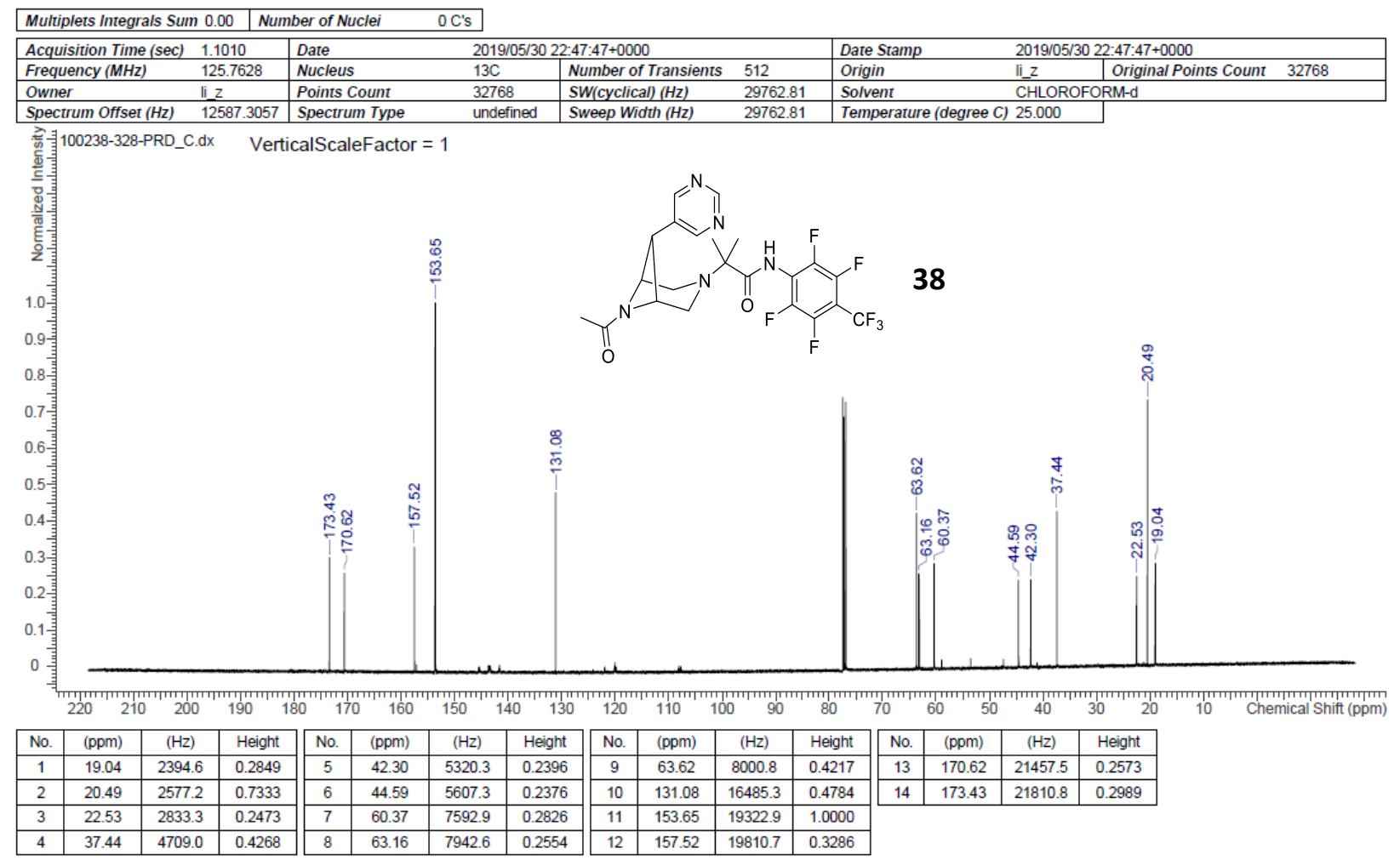

\begin{tabular}{|c|c|c|c|c|c|c|c|c|c|}
\hline Multiplets Integrals Sun & 20.91 & mber of Nuclei & $21 \mathrm{H}$ 's & & & & & & \\
\hline Acquisition Time (sec) & 1.6383 & Date & $2019 / 05 / 17$ & $5: 31: 37+0000$ & & Date Stamp & $2019 / 05$ & 15:31:37+0000 & \\
\hline Frequency $(\mathrm{MHz})$ & 500.1030 & Nucleus & $1 \mathrm{H}$ & Number of Transients & 8 & Origin & li_z & Original Points Count & 16384 \\
\hline Owner & li_z & Points Count & 16384 & SW(cyclical) $(\mathrm{Hz})$ & 10000.61 & Solvent & CHLOR & RM-d & \\
\hline Spectrum Offset $(\mathrm{Hz})$ & 2988.8655 & Spectrum Type & undefined & Sweep Width $(\mathrm{Hz})$ & 10000.61 & Temperature (degree $C$ ) & 25.000 & & \\
\hline
\end{tabular}

${ }^{1} \mathrm{H}$ NMR $(500 \mathrm{MHz}$, CHLOROFORM- $d$ ) $\delta \mathrm{ppm} 1.17$ (s, $3 \mathrm{H}) 1.22(\mathrm{~s}, 3 \mathrm{H}) 2.05$ (s, $\left.3 \mathrm{H}\right) 3.09(\mathrm{dd}, J=11.60,2.44 \mathrm{~Hz}, 1 \mathrm{H}) 3.15$ (dd, $J=11.60,2.44$ $\mathrm{Hz}, 1 \mathrm{H}) 3.23(\mathrm{dd}, J=11.60,1.22 \mathrm{~Hz}, 1 \mathrm{H}) 3.46(\mathrm{dd}, J=11.60,1.22 \mathrm{~Hz}, 1 \mathrm{H}) 4.12(\mathrm{td}, J=5.80,1.22 \mathrm{~Hz}, 1 \mathrm{H}) 4.77-4.94(\mathrm{~m}, 2 \mathrm{H}) 6.80(\mathrm{~s}, 1 \mathrm{H}) 7.36$ (dd, $J=9.16,1.22 \mathrm{~Hz}, 1 \mathrm{H}) 7.75(\mathrm{~d}, J=9.16 \mathrm{~Hz}, 1 \mathrm{H}) 8.11(\mathrm{~s}, 1 \mathrm{H}) 8.38(\mathrm{~s}, 1 \mathrm{H})$

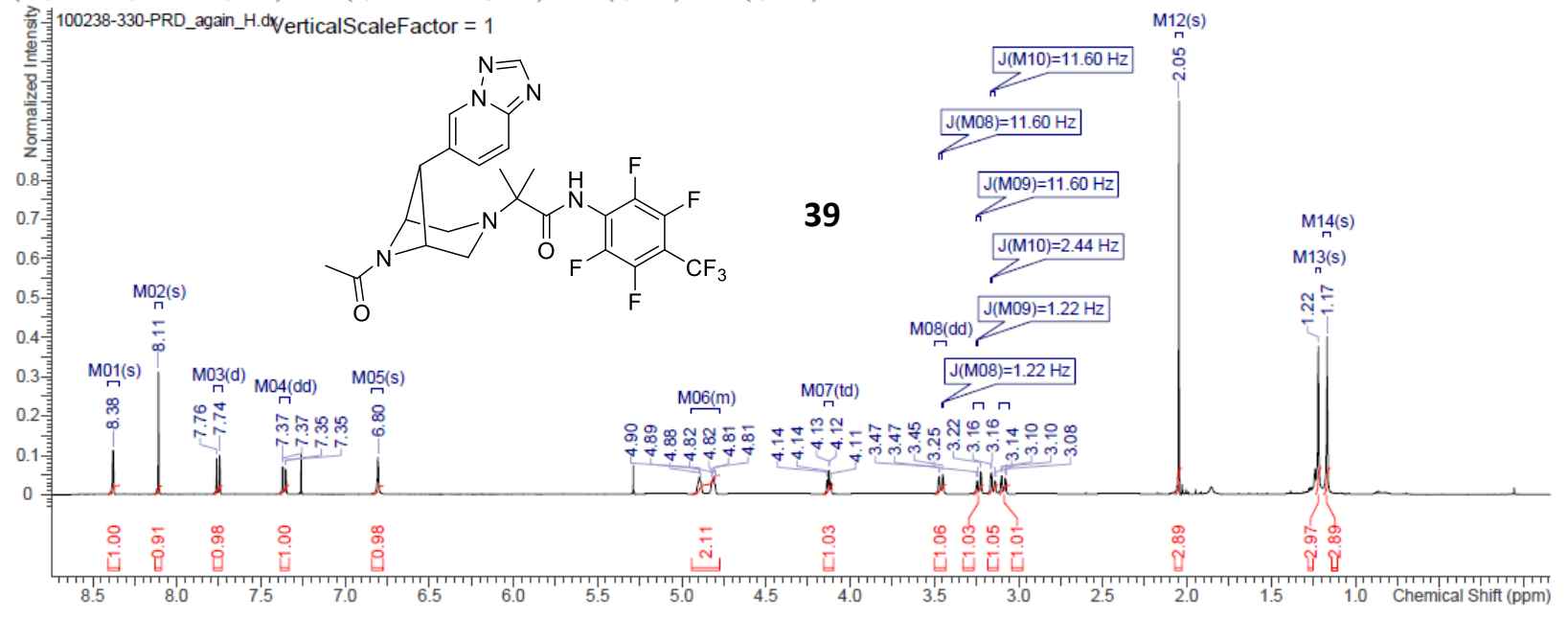

\begin{tabular}{|c|c|c|c|c|c|c|c|c|c|c|c|c|c|}
\hline No. & $\begin{array}{l}\text { Shift1 } \\
\text { (ppm) }\end{array}$ & H's & Type & $\begin{array}{c}\mathrm{J} \\
(\mathrm{Hz})\end{array}$ & Multiplet1 & (ppm) & No. & $\begin{array}{l}\text { Shift1 } \\
\text { (ppm) }\end{array}$ & H's & Type & $\begin{array}{c}\mathrm{J} \\
(\mathrm{Hz})\end{array}$ & Multiplet1 & (ppm) \\
\hline 1 & 1.17 & 3 & $\mathrm{~s}$ & - & M14 & {$\left[\begin{array}{lll}1.15 & \ldots & 1.19]\end{array}\right.$} & 8 & 4.12 & 1 & td & $5.80,1.22$ & M07 & {$\left[\begin{array}{l}4.10 \ldots 4.16] \\
0\end{array}\right.$} \\
\hline 2 & 1.22 & 3 & $\mathrm{~s}$ & - & M13 & {$\left[\begin{array}{lll}1.20 & \ldots & 1.23\end{array}\right]$} & 9 & 4.84 & 2 & $\mathrm{~m}$ & - & M06 & {$\left[\begin{array}{lll}4.77 & 4.94]\end{array}\right]$} \\
\hline 3 & 2.05 & 3 & $\mathrm{~s}$ & - & M12 & {$\left[\begin{array}{lll}2.03 & .2 .07]\end{array}\right]$} & 10 & 6.80 & 1 & $\mathrm{~s}$ & - & M05 & {$\left[\begin{array}{lll}6.77 & \ldots & 6.84]\end{array}\right]$} \\
\hline 4 & 3.09 & 1 & dd & $11.60,2.44$ & M11 & [3.05 .. 3.11] & 11 & 7.36 & 1 & dd & $9.16,1.22$ & M04 & {$\left[\begin{array}{l}7.33 \ldots . .7 .39] \\
\end{array}\right.$} \\
\hline 5 & 3.15 & 1 & dd & $11.60,2.44$ & M10 & {$[3.12 \ldots 3.18]$} & 12 & 7.75 & 1 & d & 9.16 & M03 & {$[7.73 \ldots 7.78]$} \\
\hline 6 & 3.23 & 1 & dd & $11.60,1.22$ & M09 & {$[3.21 \ldots 3.27]$} & 13 & 8.11 & 1 & $\mathrm{~s}$ & - & M02 & [8.09 . 8.13] \\
\hline 7 & 3.46 & 1 & dd & $11.60,1.22$ & M08 & {$[3.43 \ldots .3 .50]$} & 14 & 8.38 & 1 & $\mathrm{~s}$ & - & M01 & [8.34 ..8.41] \\
\hline
\end{tabular}




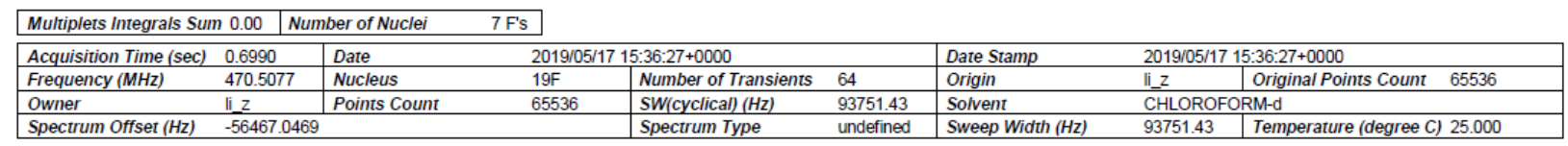

${ }^{19} \mathrm{~F}$ NMR (471 MHz, CHLOROFORM- $d$ ) $\delta$ ppm -143.79 - -143.60 (m, 2 F) $-140.54--140.25$ (m, 2 F) $-56.13(\mathrm{t}, J=21.46 \mathrm{~Hz}, 3 \mathrm{~F})$
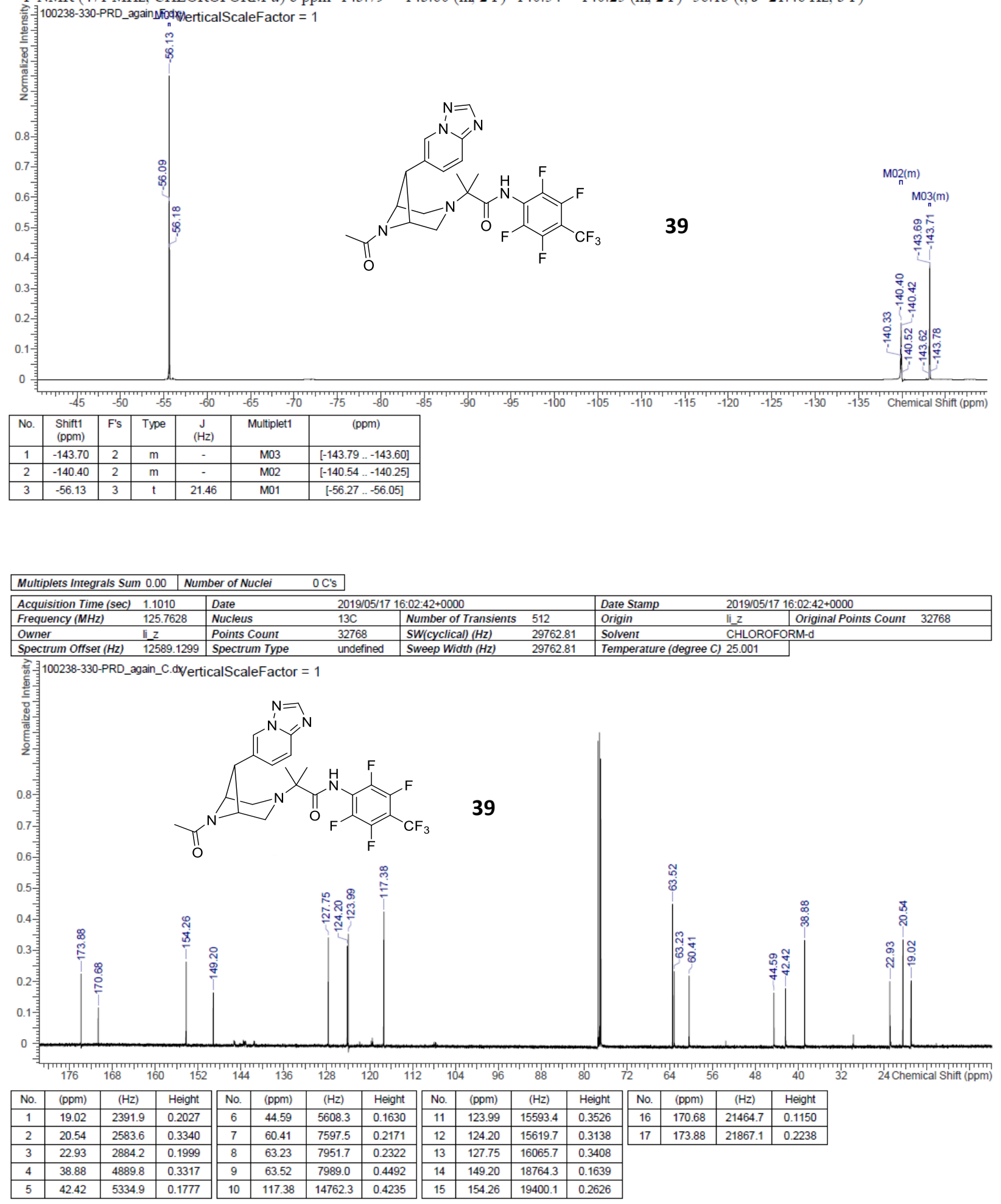

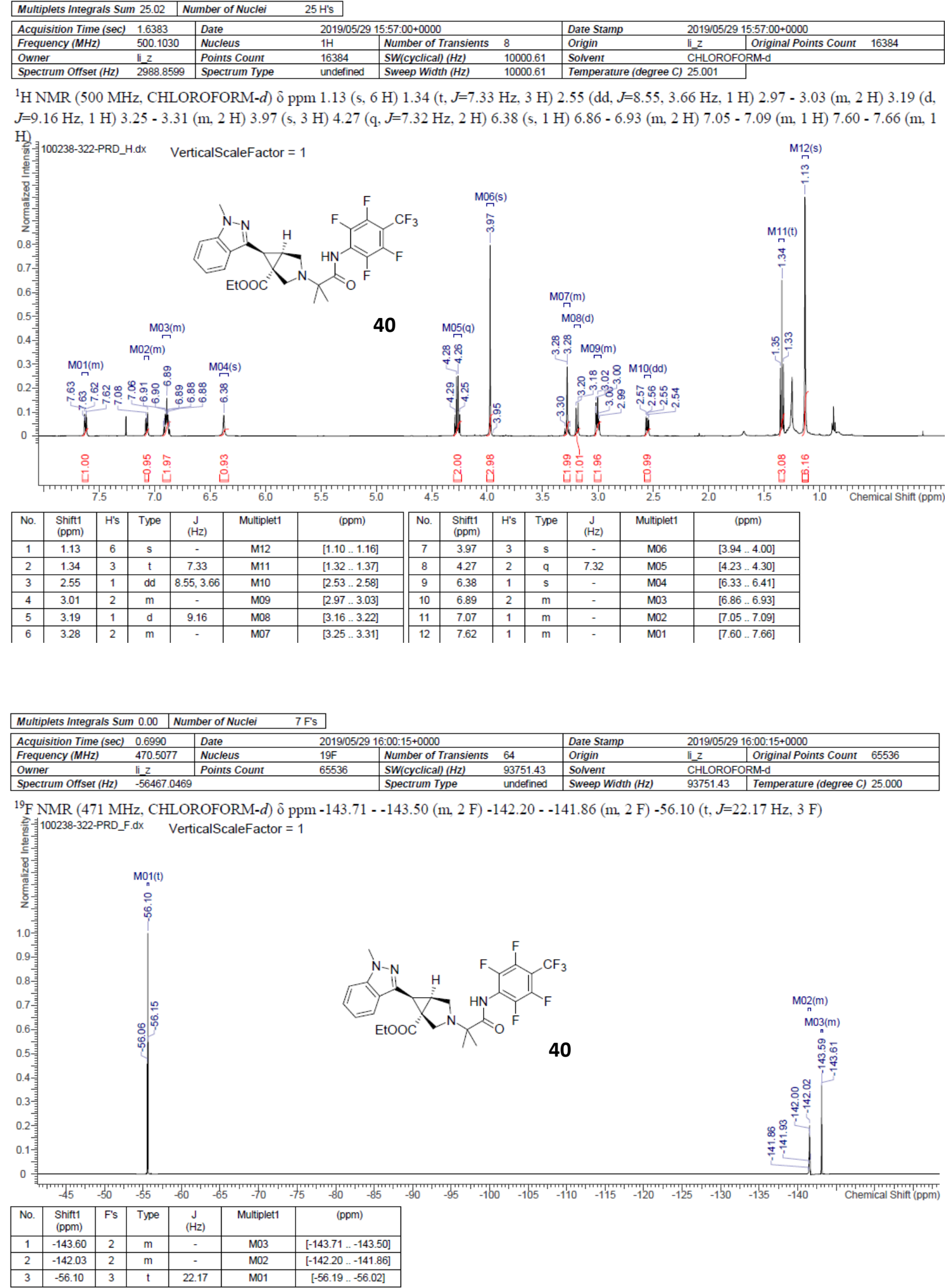

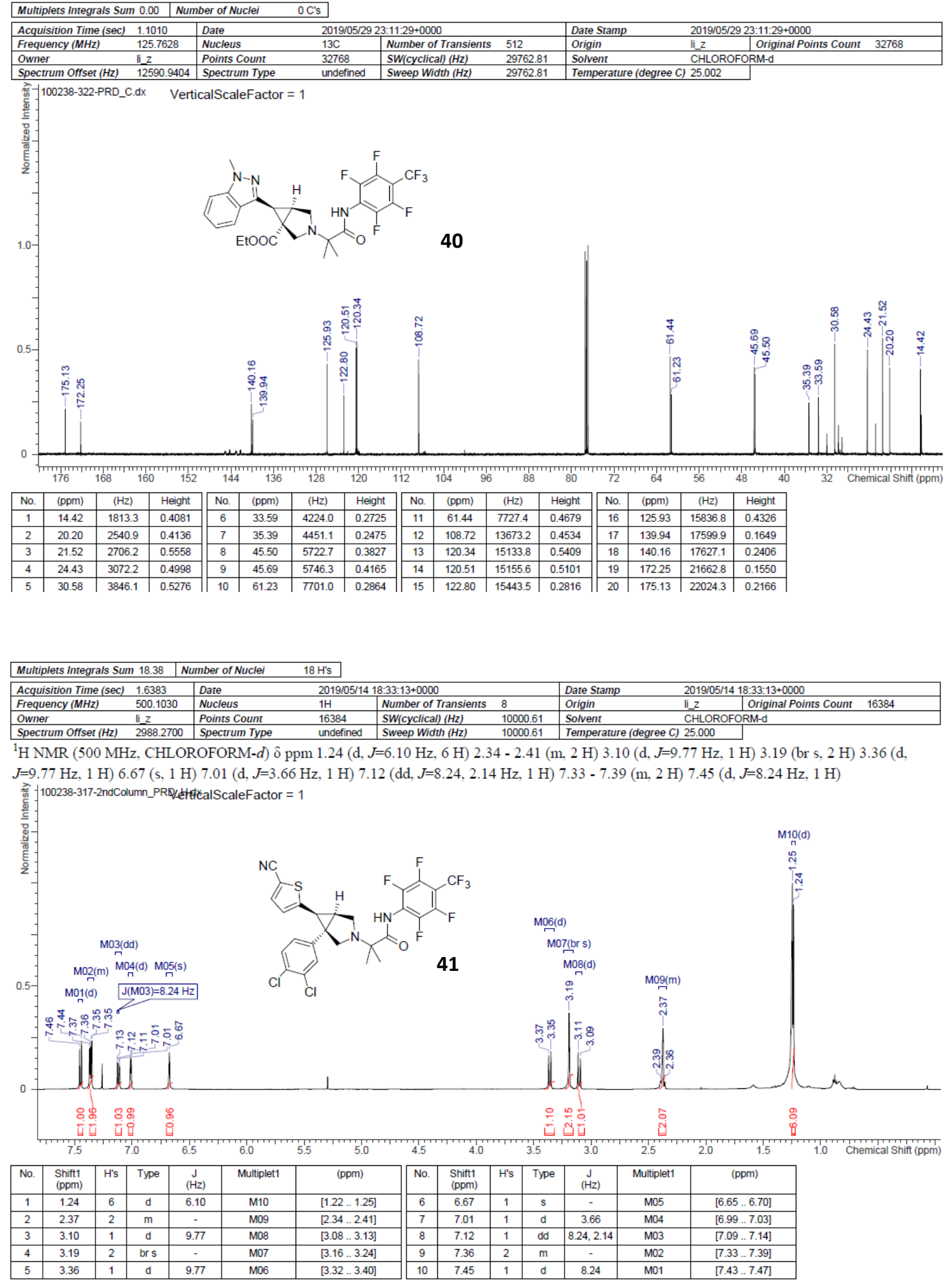

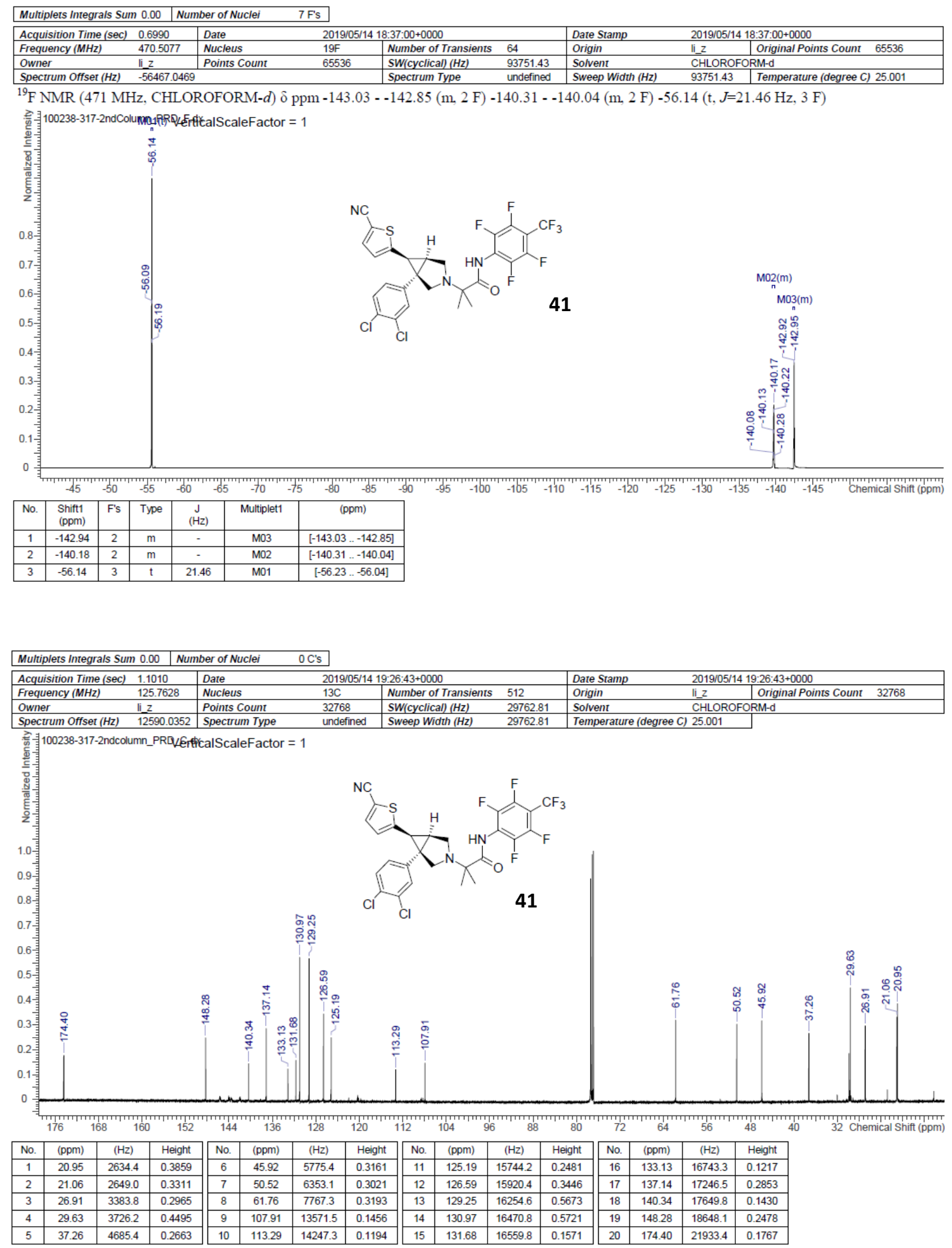


\begin{tabular}{|c|c|c|c|c|c|c|c|c|c|}
\hline Multiplets Integrals Sun & 22.24 & Vumber of Nuclei & $21 \mathrm{H}$ 's & & & & & & \\
\hline Acquisition Time (sec) & 1.6383 & Date & $2019 / 05 / 29$ & $5: 47: 41+0000$ & & Date Stamp & $2019 / 05$ & $5: 47: 41+0000$ & \\
\hline Frequency $(\mathrm{MHz})$ & 500.1030 & Nucleus & $1 \mathrm{H}$ & Number of Transients & 8 & Origin & li_z & \begin{tabular}{|l|} 
Original Points Count \\
\end{tabular} & 16384 \\
\hline Owner & li_z & Points Count & 16384 & $S W($ cyclical) $(\mathrm{Hz})$ & 10000.61 & Solvent & CHLOR & RM-d & \\
\hline Spectrum Offset $(\mathrm{Hz})$ & 2988.8752 & Spectrum Type & undefined & Sweep Width $(\mathrm{Hz})$ & 10000.61 & Temperature (degree $C$ ) & 25.002 & & \\
\hline
\end{tabular}

${ }^{1} \mathrm{H}$ NMR $(500 \mathrm{MHz}$, CHLOROFORM- $d$ ) $\delta \mathrm{ppm} 1.22(\mathrm{~d}, J=1.22 \mathrm{~Hz}, 6 \mathrm{H}) 2.17-2.26(\mathrm{~m}, 2 \mathrm{H}) 3.02(\mathrm{~d}, J=8.55 \mathrm{~Hz}, 1 \mathrm{H}) 3.09$ (dd, $J=9.16,3.05 \mathrm{~Hz}, 1$

H) $3.26(\mathrm{~d}, J=9.16 \mathrm{~Hz}, 1 \mathrm{H}) 3.42(\mathrm{~d}, J=8.55 \mathrm{~Hz}, 1 \mathrm{H}) 3.67(\mathrm{~s}, 3 \mathrm{H}) 6.17(\mathrm{~d}, J=1.83 \mathrm{~Hz}, 1 \mathrm{H}) 7.10-7.17(\mathrm{~m}, 2 \mathrm{H}) 7.36-7.42(\mathrm{~m}, 2 \mathrm{H}) 7.62(\mathrm{br} \mathrm{s}, 1 \mathrm{H})$

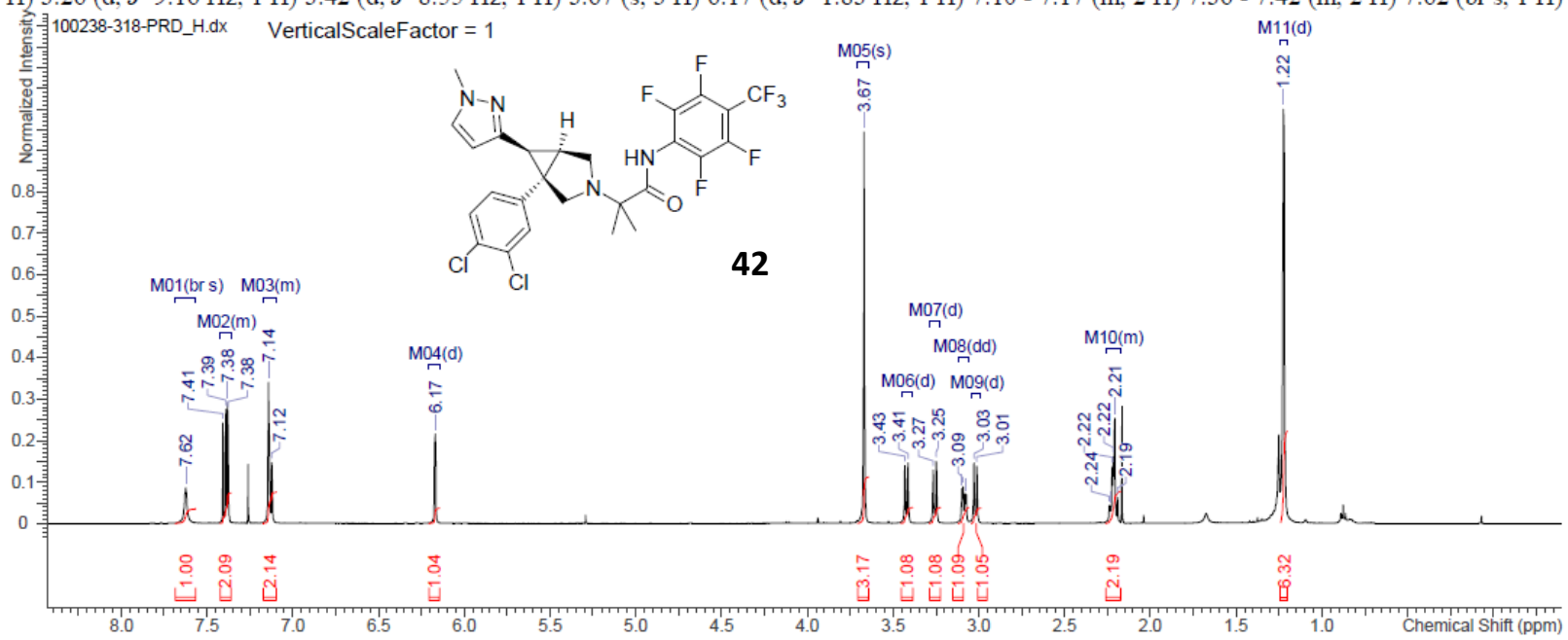

\begin{tabular}{|c|c|c|c|c|c|c|c|c|c|c|c|c|c|}
\hline No. & $\begin{array}{l}\text { Shift1 } \\
\text { (ppm) }\end{array}$ & H's & Type & $\begin{array}{c}\mathrm{J} \\
(\mathrm{Hz})\end{array}$ & Multiplet1 & (ppm) & No. & $\begin{array}{l}\text { Shift1 } \\
(\mathrm{ppm})\end{array}$ & H's & Type & $\begin{array}{c}\mathrm{J} \\
(\mathrm{Hz})\end{array}$ & Multiplet1 & (ppm) \\
\hline 1 & 1.22 & 6 & d & 1.22 & M11 & {$[1.20 \ldots 1.24]$} & 7 & 3.67 & 3 & $\mathrm{~s}$ & - & M05 & [3.64 .. 3.71] \\
\hline 2 & 2.21 & 2 & $\mathrm{~m}$ & - & M10 & {$[2.17 \ldots 2.26]$} & 8 & 6.17 & 1 & d & 1.83 & M04 & {$[6.14 \ldots 6.21]$} \\
\hline 3 & 3.02 & 1 & d & 8.55 & M09 & {$[2.99 \ldots 3.04]$} & 9 & 7.13 & 2 & $\mathrm{~m}$ & - & M03 & {$[7.10 \ldots 7.17]$} \\
\hline 4 & 3.09 & 1 & dd & $9.16,3.05$ & M08 & {$\left[\begin{array}{lll}3.06 & . & 3.12\end{array}\right]$} & 10 & 7.39 & 2 & $\mathrm{~m}$ & - & M02 & [7.36 . . 7.42] \\
\hline 5 & 3.26 & 1 & d & 9.16 & M07 & [3.22 .. 3.29] & 11 & 7.62 & 1 & brs & - & M01 & {$\left[\begin{array}{llll}7.5 .57 & . .68\end{array}\right]$} \\
\hline 6 & 3.42 & 1 & d & 8.55 & M06 & [3.39 .. 3.45] & & & & & & & \\
\hline
\end{tabular}

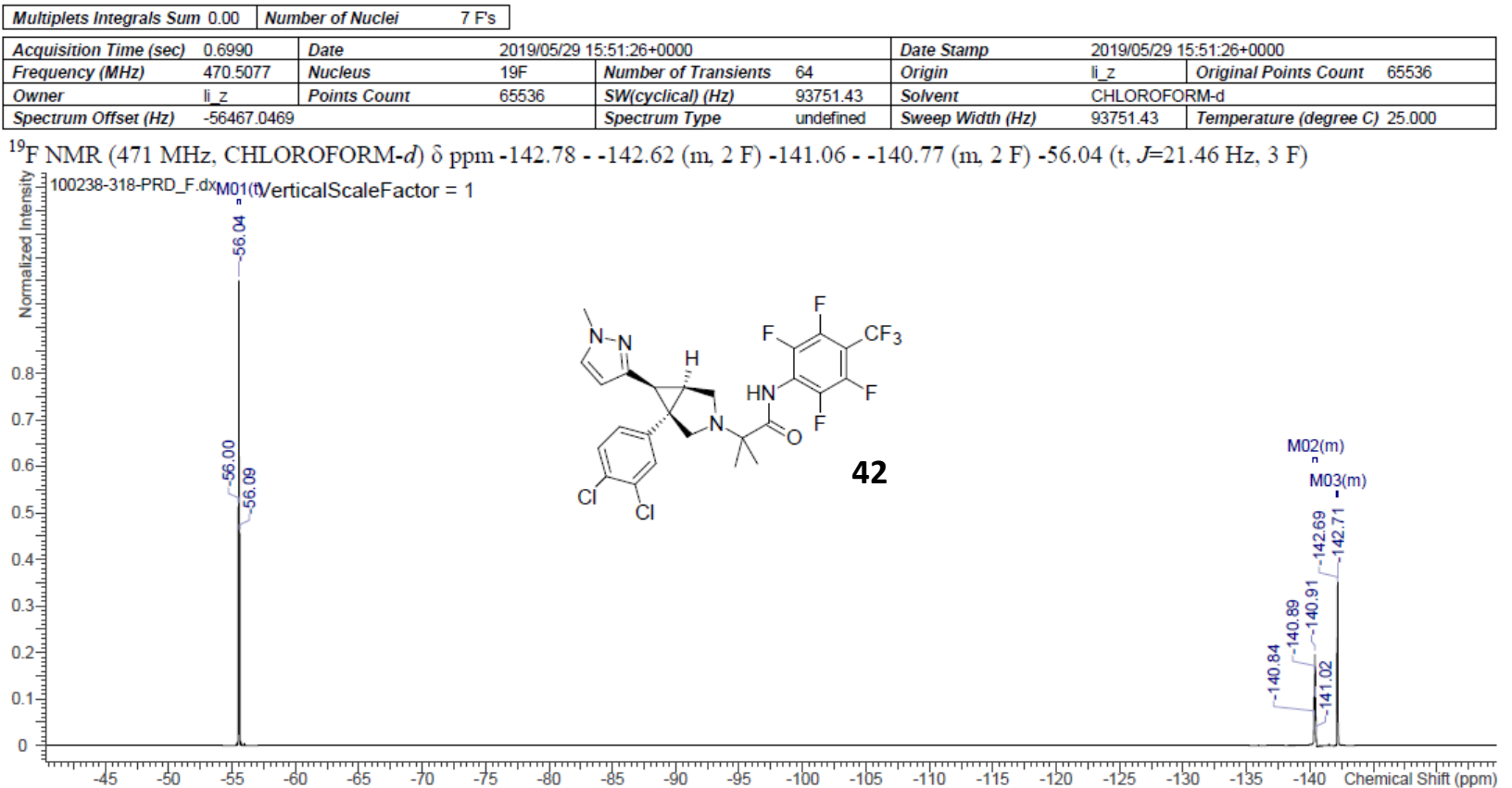

\begin{tabular}{|c|c|c|c|c|c|c|}
\hline No. & $\begin{array}{c}\text { Shift1 } \\
(\mathrm{ppm})\end{array}$ & F's & Type & $\begin{array}{c}\mathrm{J} \\
(\mathrm{Hz})\end{array}$ & Multiplet1 & $(\mathrm{ppm})$ \\
\hline 1 & -142.70 & 2 & $\mathrm{~m}$ & - & M03 & {$[-142.78 \ldots-142.62]$} \\
\hline 2 & -140.92 & 2 & $\mathrm{~m}$ & - & M02 & {$[-141.06 \ldots-140.77]$} \\
\hline 3 & -56.04 & 3 & $\mathrm{t}$ & 21.46 & M01 & {$[-56.13 \ldots-55.96]$} \\
\hline
\end{tabular}




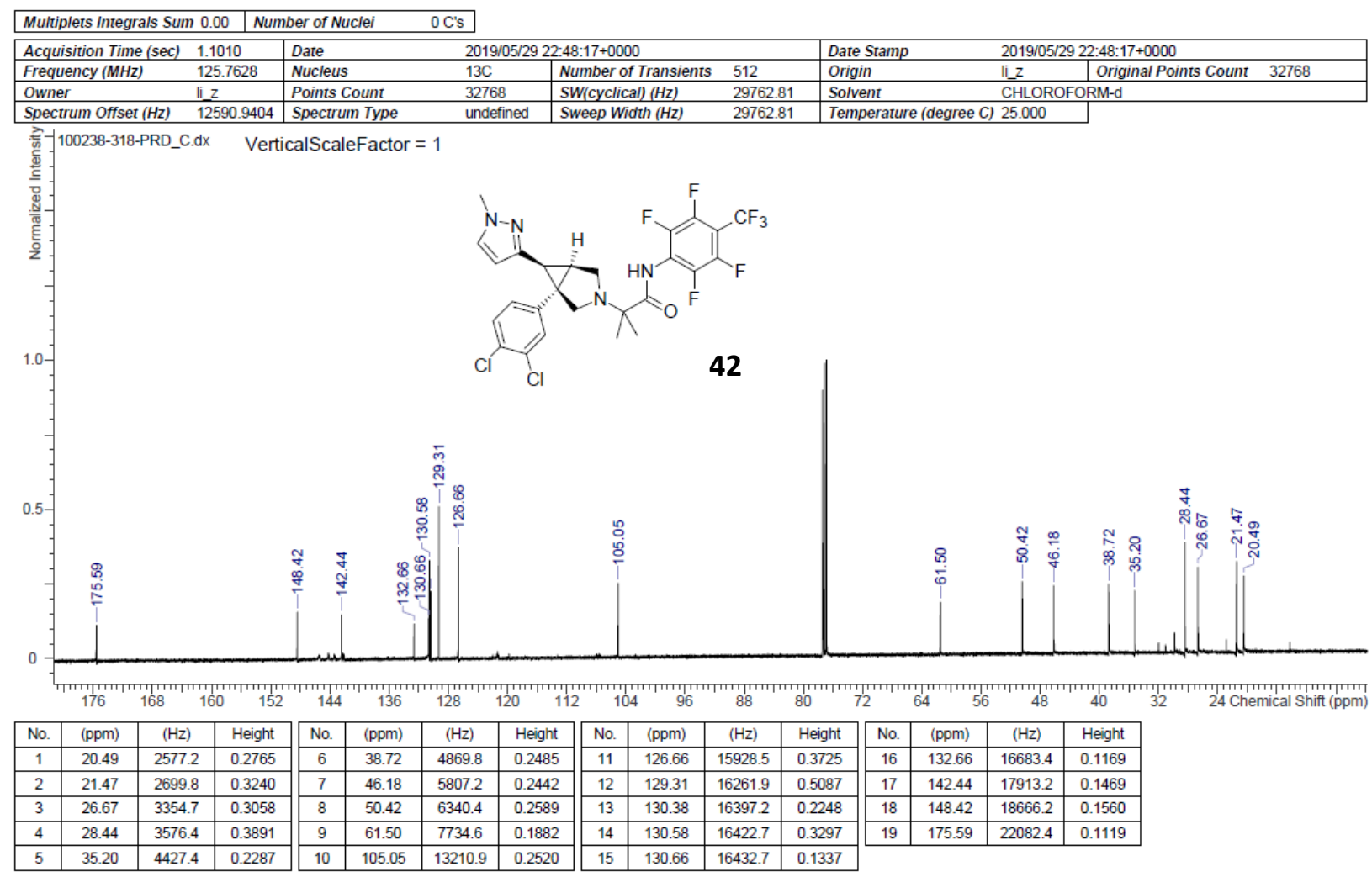

\begin{tabular}{|l|l|}
\hline Multiplets Integrals Sum 22.80 & Number of Nuclei $22 \mathrm{H}$ 's \\
\hline
\end{tabular}

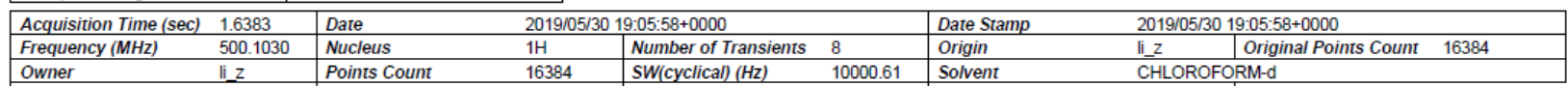

\begin{tabular}{|lllll|l|l|}
\hline Spectrum Offset $(\mathrm{Hz})$ & 2988.8804 & Spectrum Type & undefined & Sweep Width $(\mathrm{Hz})$ & 10000.61 & Temperature (degree C) 25.000 \\
\hline
\end{tabular}

${ }^{1} \mathrm{H}$ NMR (500 MHz, CHLOROFORM-d) $\delta \mathrm{ppm} 1.17$ (d, J=5.49 Hz, $\left.6 \mathrm{H}\right) 2.39-2.47$ (m, $\left.2 \mathrm{H}\right) 3.09$ (d, J=9.16 Hz, $\left.1 \mathrm{H}\right) 3.14$ (dd, J=9.16, $4.27 \mathrm{~Hz}, 1$ H) $3.30(\mathrm{~d}, J=9.16 \mathrm{~Hz}, 1 \mathrm{H}) 3.46(\mathrm{~d}, J=9.16 \mathrm{~Hz}, 1 \mathrm{H}) 4.01(\mathrm{~s}, 3 \mathrm{H}) 6.69(\mathrm{~s}, 1 \mathrm{H}) 6.92(\mathrm{dd}, J=7.94,4.58 \mathrm{~Hz}, 1 \mathrm{H}) 7.22(\mathrm{dd}, J=7.94,1.83 \mathrm{~Hz}, 1 \mathrm{H}) 7.44$ - $7.48(\mathrm{~m}, 2 \mathrm{H}) 7.97$ (dd, $J=7.94,1.22 \mathrm{~Hz}, 1 \mathrm{H}) 8.13(\mathrm{dd}, J=4.58,1.22 \mathrm{~Hz}, 1 \mathrm{H})$



\begin{tabular}{|c|c|c|c|c|c|c|c|c|c|c|c|c|c|}
\hline No. & $\begin{array}{l}\text { Shift1 } \\
(\mathrm{ppm})\end{array}$ & H's & Type & $\begin{array}{c}\mathrm{J} \\
(\mathrm{Hz})\end{array}$ & Multiplet1 & (ppm) & No. & $\begin{array}{l}\text { Shift1 } \\
\text { (ppm) }\end{array}$ & H's & Type & $\underset{(\mathrm{Hz})}{\mathrm{J}}$ & Multiplet1 & (ppm) \\
\hline 1 & 1.17 & 6 & d & 5.49 & M13 & {$[1.14 \ldots 1.19]$} & 8 & 6.69 & 1 & $\mathrm{~s}$ & - & M06 & {$\left[\begin{array}{lll}6.65 & .6 .72\end{array}\right]$} \\
\hline 2 & 2.43 & 2 & $\mathrm{~m}$ & - & M12 & {$[2.39 \ldots 2.47]$} & 9 & 6.92 & 1 & dd & \begin{tabular}{|l|}
$7.94,4.58$ \\
\end{tabular} & M05 & {$\left[\begin{array}{ll}6.90 & .6 .95]\end{array}\right.$} \\
\hline 3 & 3.09 & 1 & d & 9.16 & M11 & {$[3.06 \ldots 3.10]$} & 10 & 7.22 & 1 & dd & $7.94,1.83$ & M04 & {$[7.20 \ldots 7.24]$} \\
\hline 4 & 3.14 & 1 & $d d$ & \begin{tabular}{|l|}
$9.16,4.27$ \\
\end{tabular} & M10 & {$[3.12 \ldots 3.17]$} & 11 & 7.46 & 2 & $\mathrm{~m}$ & - & M03 & {$[7.44 \ldots 7.48]$} \\
\hline 5 & 3.30 & 1 & d & 9.16 & M09 & {$[3.28 \ldots .3 .33]$} & 12 & 7.97 & 1 & dd & $7.94,1.22$ & M02 & [7.95 .. 7.99] \\
\hline 6 & 3.46 & 1 & d & 9.16 & M08 & {$[3.43 \ldots 3.48]$} & 13 & 8.13 & 1 & dd & \begin{tabular}{|l|}
$4.58,1.22$ \\
\end{tabular} & M01 & {$[8.11 \ldots 8.15]$} \\
\hline 7 & 4.01 & 3 & $\mathrm{~s}$ & - & M07 & {$[3.99 \ldots 4.03]$} & & & & & & & \\
\hline
\end{tabular}



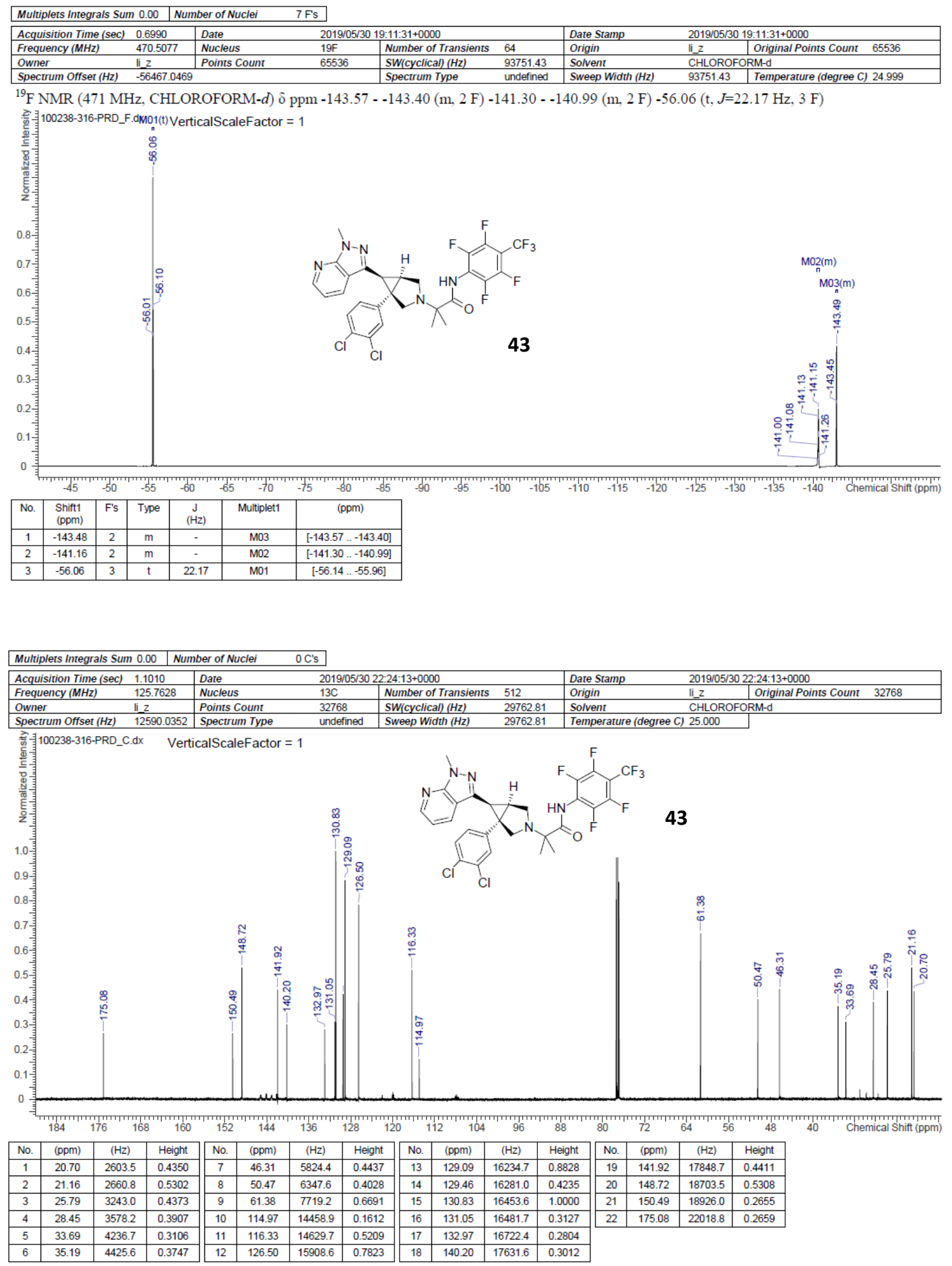

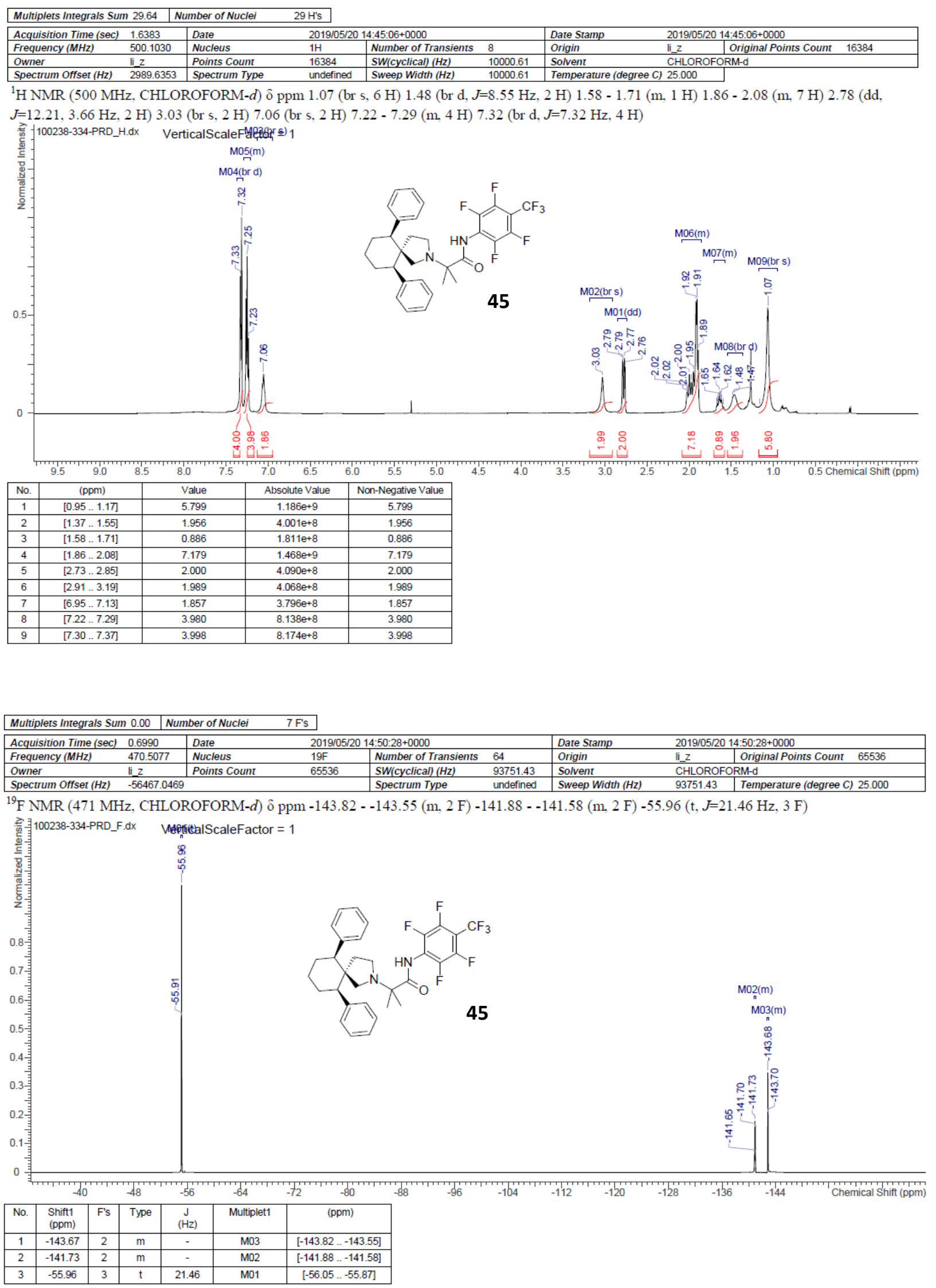


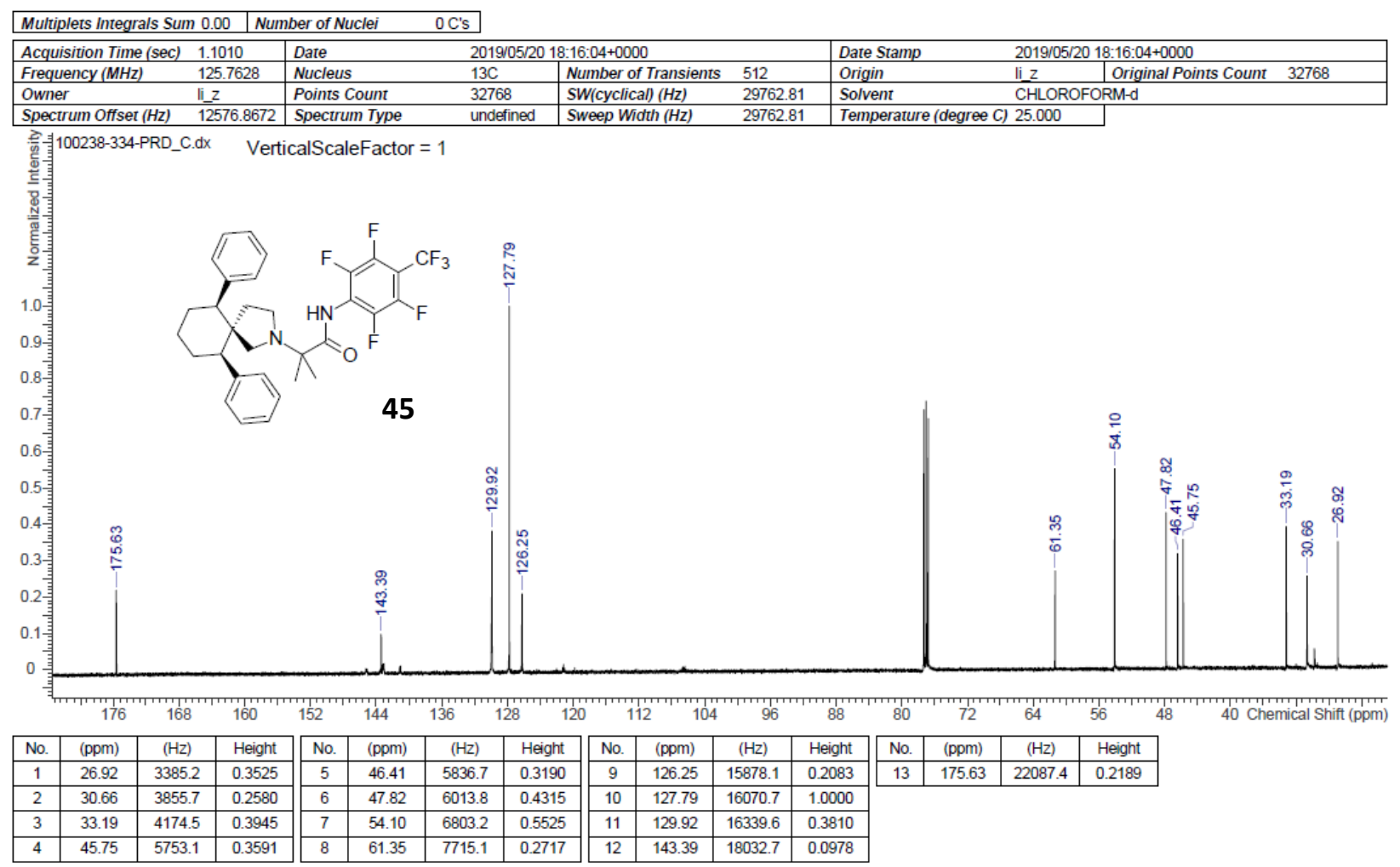

\begin{tabular}{|c|c|c|c|c|c|c|c|}
\hline \multicolumn{2}{|c|}{ Multiplets Integrals Sum 19.42} & Number of Nuclej & $19 \mathrm{H}$ 's & & & & \\
\hline Acquisition Time (sec) & \multirow{2}{*}{\multicolumn{2}{|c|}{$2018 / 08 / 2418: 18: 21+0000$}} & \multicolumn{5}{|c|}{ BIIB 1D proton short $\mathrm{CDCl}$ \{C:IBruker|TopSpin3.0\} li z 60} \\
\hline Date & & & \multirow{3}{*}{$\frac{1 \mathrm{H}}{\mathrm{li} Z}$} & \multirow{2}{*}{$\begin{array}{l}\text { Date Stamp } \\
\text { Number of Transients }\end{array}$} & \multicolumn{3}{|c|}{$2018 / 08 / 2418: 18: 21+0000$} \\
\hline Frequency $(\mathrm{MHz})$ & \multicolumn{2}{|c|}{\begin{tabular}{|l|l}
500.1030 & Nucleus \\
\end{tabular}} & & & 8 & Origin & liz \\
\hline Original Points Count & 16384 & Owner & & \begin{tabular}{|l|} 
Points Count \\
\end{tabular} & 16384 & SW(cyclical) $(\mathrm{Hz})$ & 10000.61 \\
\hline Solvent & CHLORC & FORM-d & & Spectrum Offset $(\mathrm{Hz})$ & 2993.8760 & Spectrum Type & undefined \\
\hline
\end{tabular}

${ }^{1} \mathrm{H}$ NMR $(500 \mathrm{MHz}$, CHLOROFORM- $d$ ) $\delta$ ppm $1.87-1.93(\mathrm{~m}, 2 \mathrm{H}) 2.04(\mathrm{t}, J=7.94 \mathrm{~Hz}, 1 \mathrm{H}) 2.43(\mathrm{~s}, 3 \mathrm{H}) 3.28-3.33$ (m, $2 \mathrm{H}) 3.58$ (d, J=9.16 Hz, $2 \mathrm{H}) 3.81(\mathrm{~s}, 3 \mathrm{H}) 6.09$ (d, J=1.83 Hz, $1 \mathrm{H}) 7.20(\mathrm{~d}, J=1.83 \mathrm{~Hz}, 1 \mathrm{H}) 7.25(\mathrm{~d}, J=7.94 \mathrm{~Hz}, 2 \mathrm{H}) 7.51$ (d, J=7.94 Hz, $2 \mathrm{H})$
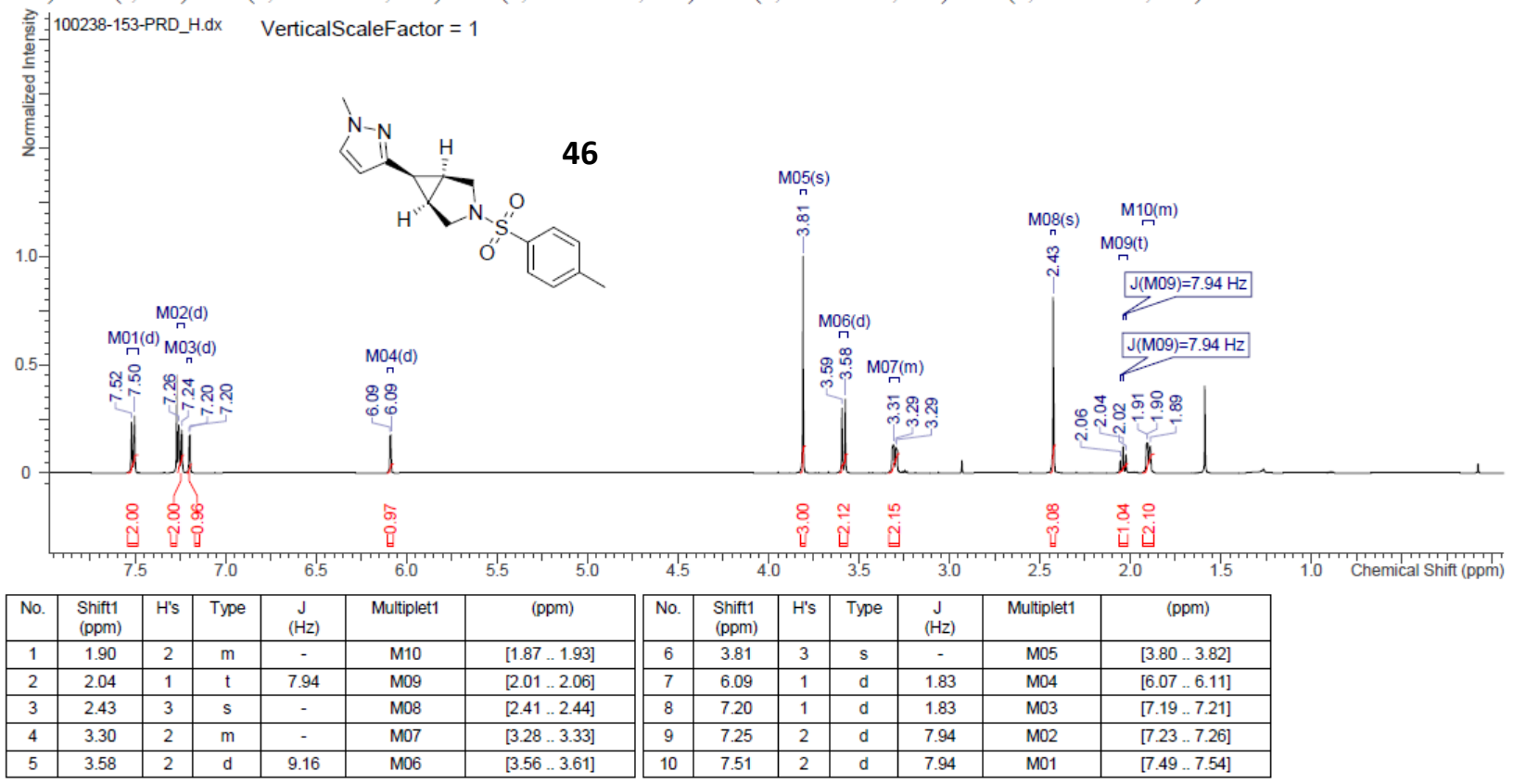

\begin{tabular}{|c|c|c|c|c|c|c|c|c|c|c|c|c|c|}
\hline No. & $\begin{array}{l}\text { Shift1 } \\
\text { (ppm) }\end{array}$ & H's & Type & $\begin{array}{c}\mathrm{J} \\
(\mathrm{Hz})\end{array}$ & Muttiplet1 & (ppm) & No. & $\begin{array}{l}\text { Shift1 } \\
\text { (ppm) }\end{array}$ & H's & Type & $\begin{array}{c}\mathrm{J} \\
(\mathrm{Hz})\end{array}$ & Multiplet1 & (ppm) \\
\hline 1 & 1.90 & 2 & $\mathrm{~m}$ & - & M10 & {$\left[\begin{array}{ll}1.87 & \ldots 1.93] \\
\end{array}\right.$} & 6 & 3.81 & 3 & s & - & MO5 & {$[3.80 \ldots 3.82]$} \\
\hline 2 & 2.04 & 1 & $t$ & 7.94 & M09 & {$[2.01 \ldots 2.06]$} & 7 & 6.09 & 1 & d & 1.83 & M04 & {$\left[\begin{array}{l}6.07 \ldots 6.11] \\
\end{array}\right.$} \\
\hline 3 & 2.43 & 3 & $\mathrm{~s}$ & - & M08 & {$[2.41 \ldots 2.44]$} & 8 & 7.20 & 1 & $\mathrm{~d}$ & 1.83 & M03 & {$[7.19 \ldots . .21]$} \\
\hline 4 & 3.30 & 2 & $\mathrm{~m}$ & - & M07 & {$[3.28 \ldots . .3 .33]$} & 9 & 7.25 & 2 & $\mathrm{~d}$ & 7.94 & M02 & {$[7.23 \ldots 7.26]$} \\
\hline 5 & 3.58 & 2 & d & 9.16 & M06 & {$[3.56$. 3.61$]$} & 10 & 7.51 & 2 & d & 7.94 & M01 & {$[7.49 \ldots 7.54]$} \\
\hline
\end{tabular}






